University of Louisville

ThinkIR: The University of Louisville's Institutional Repository

$5-2005$

\title{
A comparative study of the physical mechanisms related to highway noise barrier insertion loss : measurements versus modeling assumptions.
}

Ning Shu 1975-

University of Louisville

Follow this and additional works at: https://ir.library.louisville.edu/etd

\section{Recommended Citation}

Shu, Ning 1975-, "A comparative study of the physical mechanisms related to highway noise barrier insertion loss : measurements versus modeling assumptions." (2005). Electronic Theses and Dissertations. Paper 1323.

https://doi.org/10.18297/etd/1323

This Doctoral Dissertation is brought to you for free and open access by ThinkIR: The University of Louisville's Institutional Repository. It has been accepted for inclusion in Electronic Theses and Dissertations by an authorized administrator of ThinkIR: The University of Louisville's Institutional Repository. This title appears here courtesy of the author, who has retained all other copyrights. For more information, please contact thinkir@louisville.edu. 


\title{
A COMPARATIVE STUDY OF THE PHYSICAL MECHANISMS RELATED TO HIGHWAY NOISE BARRIER INSERTION LOSS - MEASUREMENTS VERSUS MODELING ASSUMPTIONS
}

\section{By}

\section{Ning Shu}

B.S., South China University of Technology, 1998

M.S., South China University of Technology, 2001

\author{
A Dissertation \\ Submitted to the Faculty of the \\ Graduate School of the University of Louisville \\ In Partial Fulfillment of the Requirements \\ For the Degree of
}

Doctor of Philosophy

Department of Civil and Environmental Engineering

University of Louisville

Louisville, Kentucky

May 2005 


\section{A COMPARATIVE STUDY OF THE PHYSICAL MECHANISMS}

RELATED TO HIGHWAY NOISE BARRIER INSERTION LOSS

- MEASUREMENTS VERSUS MODELING ASSUMPTIONS

\section{By}

Ning Shu

B.S., South China University of Technology, 1998

M.S., South China University of Technology, 2001

A Dissertation Approved on

April 25, 2005

by the following Dissertation Committee:

Dissertation Director 


\section{DEDICATION}

This dissertation is dedicated to

my parents

$\&$

my family

who have supported me with never ending educational opportunities. 


\section{ACKNOWLEDGEMENTS}

First and foremost, I would like to cordially thank my advisor Dr. Louis F. Cohn for his endless guidance and financial support during my entire research work. In addition, I would like to express my gratitude to Dr. Roswell A. Harris, for his academic guidance throughout my entire research work. I would also like to thank the other committee members, Dr. J.P. Mohsen, Dr. Mark French and Dr. Ahmed Desoky, for their comments and assistance during my research work.

In addition, I would like to thank Dr. Teak-Keun Kim for his comments and help. I would also like to thank Leanne Whitney, Gail Graves, Bernie Miles for their help.

Finally, I would like to express my special thanks to my parents, for their infinite support. I am further appreciative of my wife, Yufang, for her patience and continued support. Without all their unlimited understanding, this dissertation could not have been done successfully. 


\begin{abstract}
A COMPARATIVE STUDY OF THE PHYSICAL MECHANISMS RELATED TO HIGHWAY NOISE BARRIER INSERTION LOSS

- MEASUREMENTS VERSUS MODELING ASSUMPTIONS
\end{abstract}

Ning Shu

May 3, 2005

The main purpose of this research is to make a comparative study of the physical mechanisms related to highway noise barrier insertion loss and to evaluate and improve the accuracy of insertion loss of FHWA Traffic Noise Model (TNM) 2.5 compared with STAMINA 2.0, HNP 1.0 and the field measurement data. As described in Chapter VI, field measurements of highway noise barrier insertion loss were carried out along the I-264. Results show that TNM 2.5 over-predicts the insertion loss for receivers with distances up to $122 \mathrm{~m}$ ( $400 \mathrm{ft}$ ). To identify the reasons that TNM 2.5 over-predicts the insertion loss from the perspective of noise diffraction and propagation theories, new software, Highway Noise Predictor (HNP) 1.0, was designed with modified diffraction and propagation theories contained in the TNM Technical Manual and related sources.

The following findings were made based on the research: (i) STAMINA 2.0 and HNP 1.0 predict more accurate insertion loss than TNM 2.5 compared with the measurement data. (ii) TNM 2.5 over-predicts the insertion loss by about $3.0 \mathrm{dBA}$ compared with STAMINA 2.0, from the perspective of diffraction theories. (iii) TNM 2.5 
over-predicts the insertion loss by about $2.5 \mathrm{dBA}$ compared to HNP 1.0 , from the perspective of diffraction and propagation theories.

Recommendations are proposed to improve the accuracy of predicted insertion loss in TNM 2.5, as described in Chapter IX. Since noise barriers are expensive to build, more accurate prediction of insertion loss by $1.0 \mathrm{dBA}$, not only means people can be protected by noise barriers with expected satisfaction, but also a significant cost saving potential can be achieved. 
TABLE OF CONTENTS

PAGE

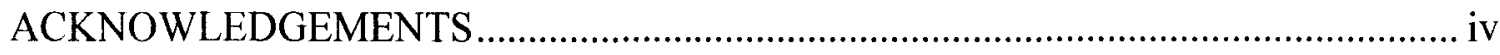

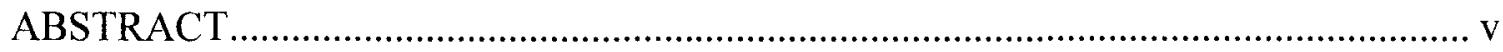

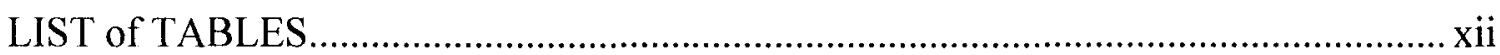

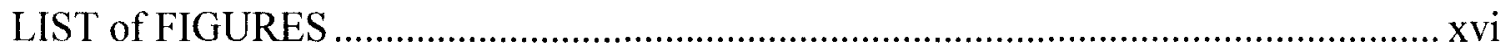

CHAPTER

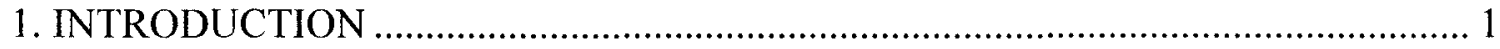

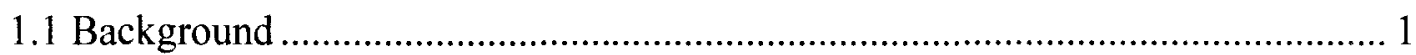

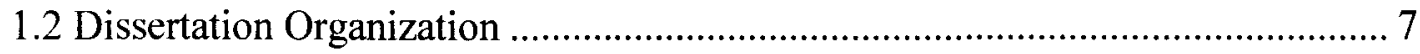

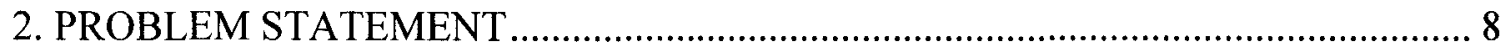

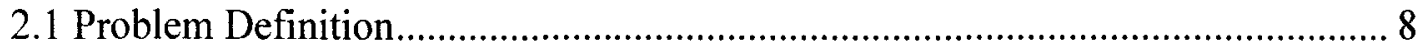

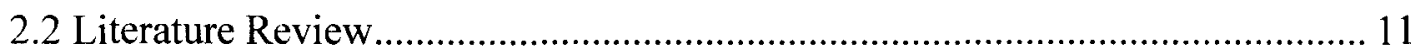

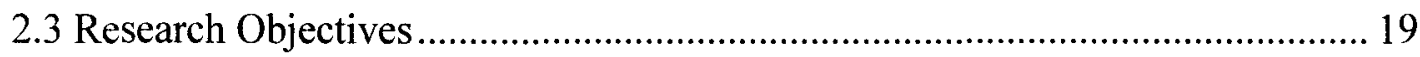

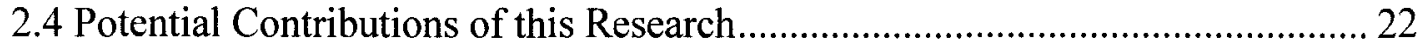

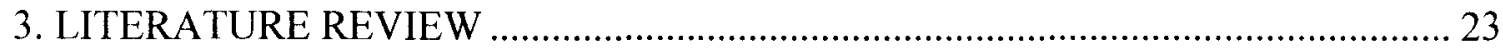

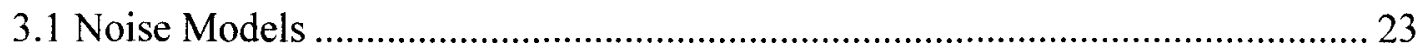

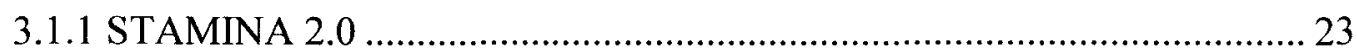

3.1.1.1 Reference Energy Mean Emission Level (REMEL) …….............. 25

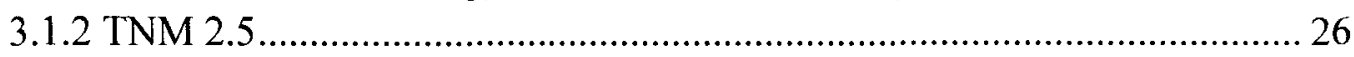

3.1.2.1 Traffic Flow Adjustment.............................................................. 29 
3.1.2.2 Distance and Roadway Length Adjustment...................................... 29

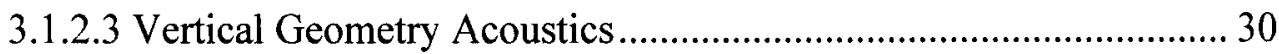

3.1.2.4 Basis of the Acoustical Model ......................................................... 30

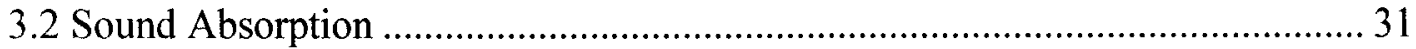

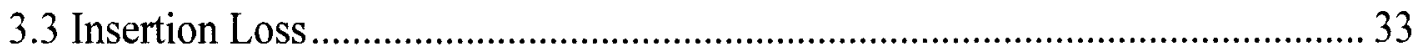

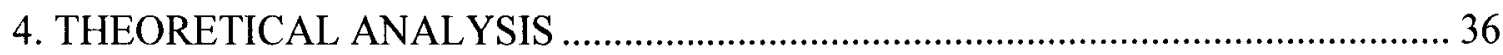

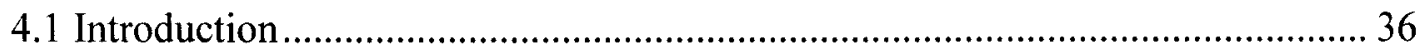

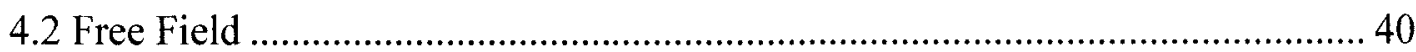

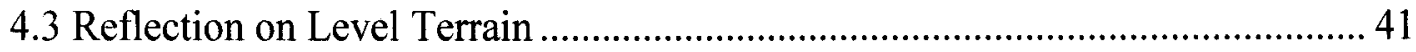

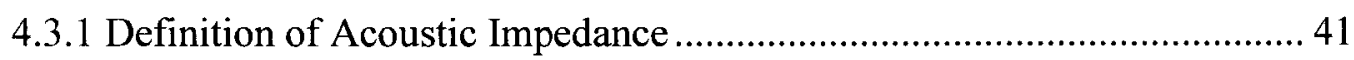

4.3.2 Impedance Models ............................................................................. 42

4.3.3 Plane Wave Reflection Coefficient........................................................... 46

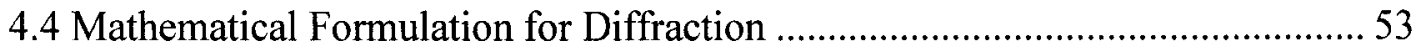

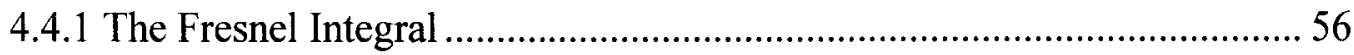

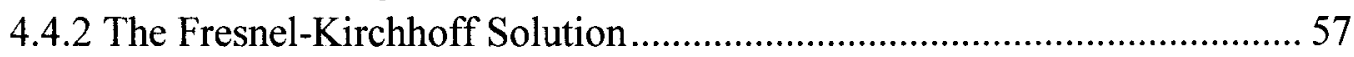

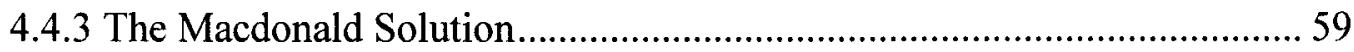

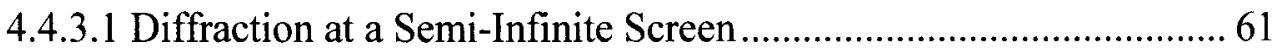

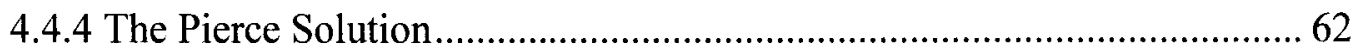

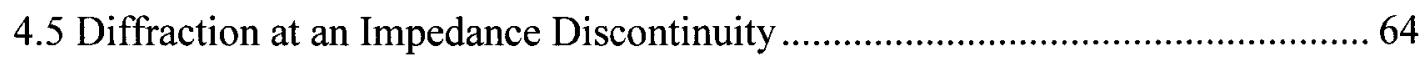

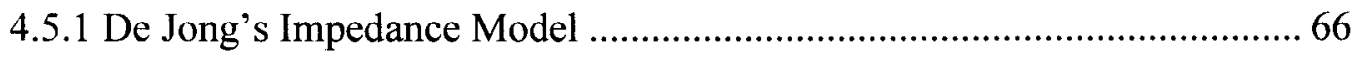

4.5.2 The Fresnel-Zone Model.................................................................... 70

4.5.3 Rasmussen's Impedance Model............................................................... 73

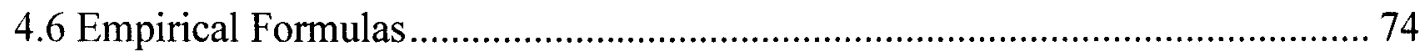

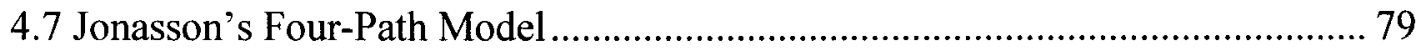

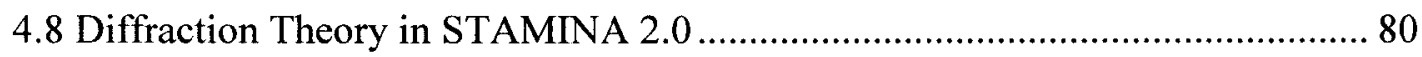

4.9 Diffraction Theory and Propagation Theory in TNM 2.5 ............................ 82

4.9.1 Propagation Path Calculations and Mathematical Description.................. 82

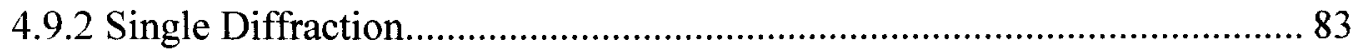

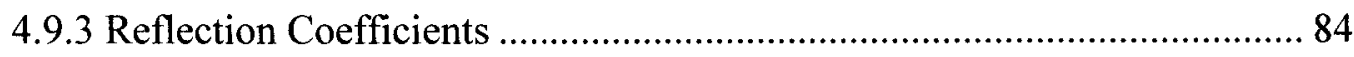




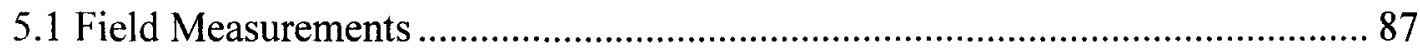

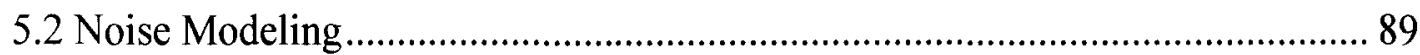

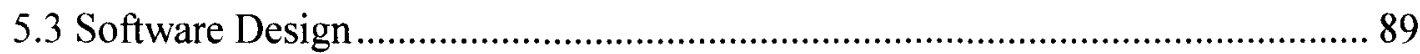

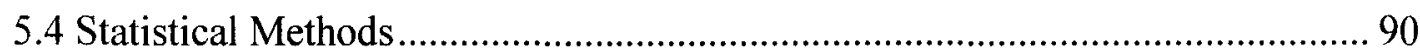

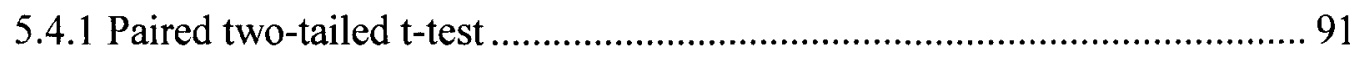

6. FIELD MEASUREMENTS OF HIGHWAY BARRIER INSERTION LOSS.............93

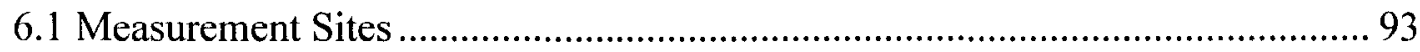

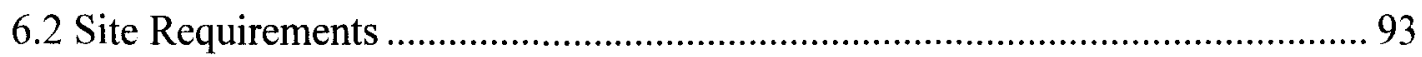

6.2.1 Site $01 \mathrm{KY}$

6.2.2 Site $02 \mathrm{KY}$

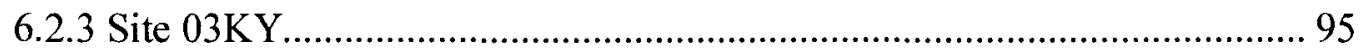

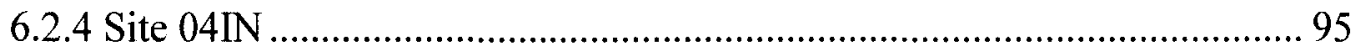

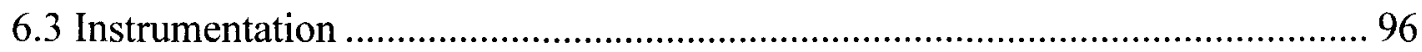

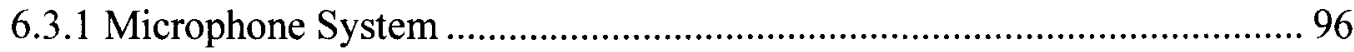

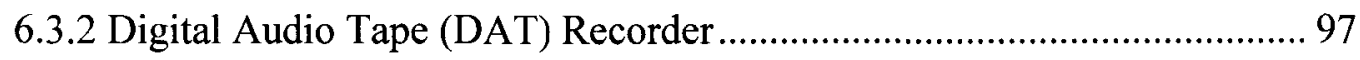

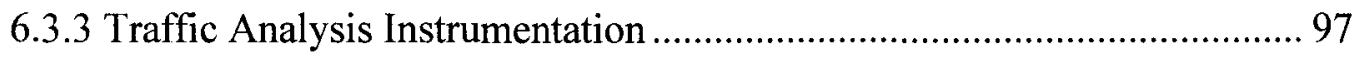

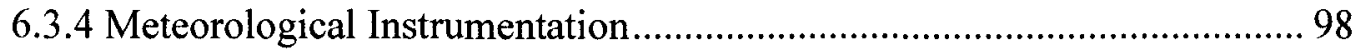

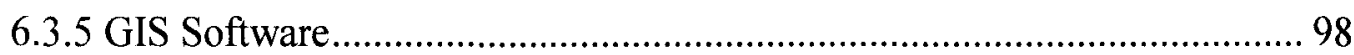

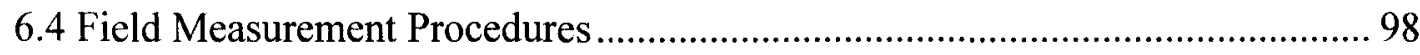

6.4.1 Measurement System Setup ................................................................... 99

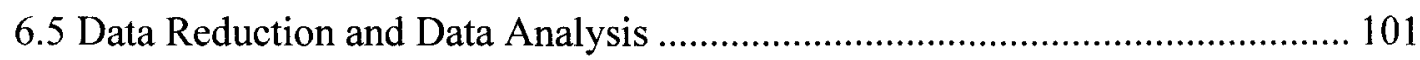

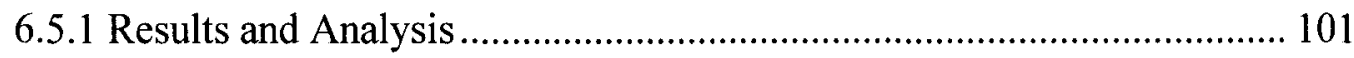

6.5.1.1 Measurement of Absolute Sound Levels ........................................ 101

6.5.1.2 Computer Modeling of Absolute Sound Levels ............................ 102

6.5.1.3 Insertion Loss Calculation ............................................................. 103

6.5.1.4 Adjusted Insertion Loss Calculation............................................. 104

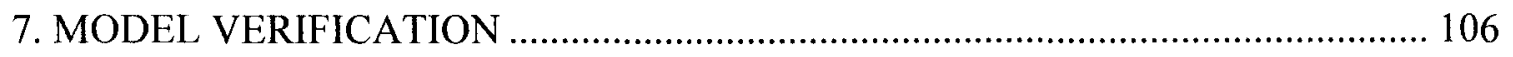


7.1 Verification of TNM Validation Cases with a Point Source ........................... 106

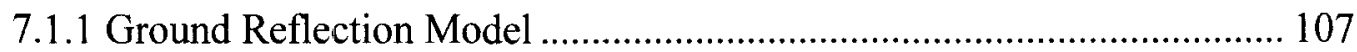

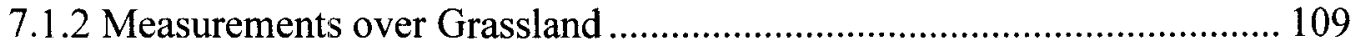

7.1.3 Comparisons of Barrier Insertion Loss .................................................. 117

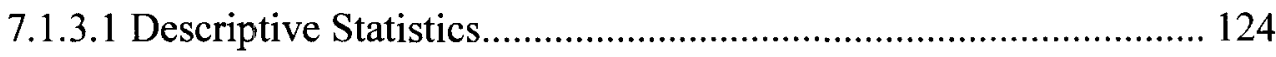

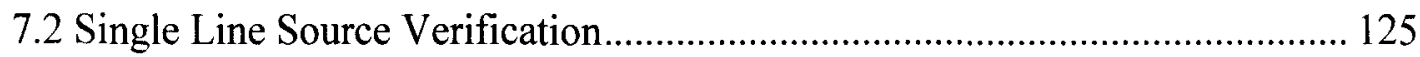

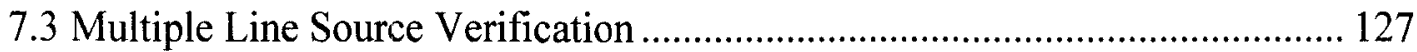

7.4 Comparison of Diffraction Theory in TNM 2.5 and STAMINA $2.0 \ldots \ldots \ldots \ldots \ldots . .128$

7.5 Effect on Insertion Loss by Reduction from Four Propagation

Paths to Two Propagation Paths....................................................................... 145

7.5.1 Comparisons of Varying Distances for the Receiver Position................. 147

7.5.2 Comparisons of Varying Heights for the Receiver Position.................... 153

8. VALIDATION WITH THE FIELD MEASUREMENTS ......................................... 162

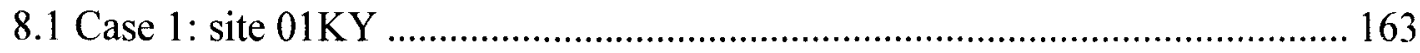

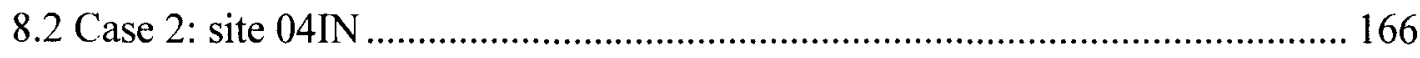

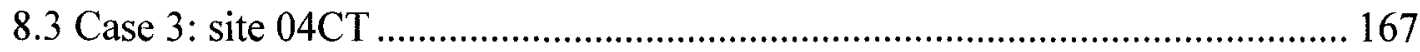

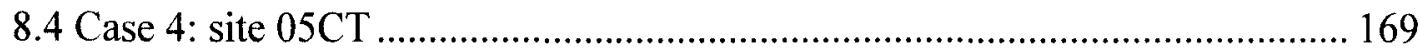

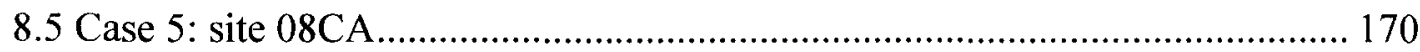

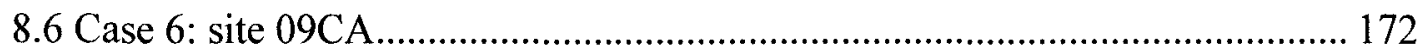

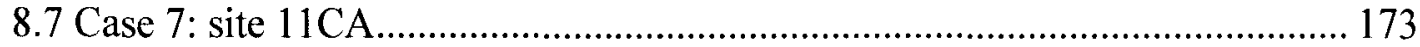

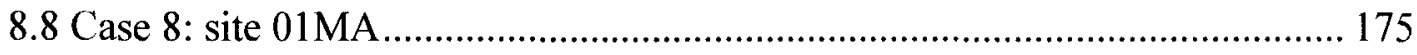

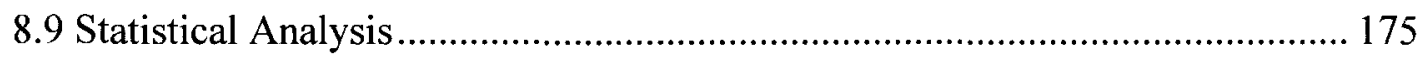

9. CONCLUSIONS AND RECOMMENDATIONS ……............................................. 178

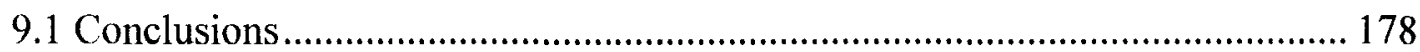

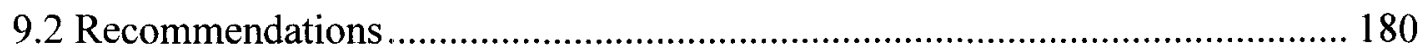


9.3 Future Research Needs.

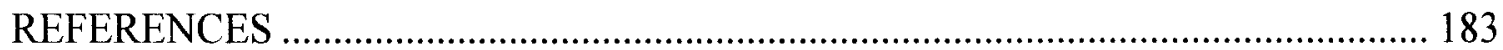

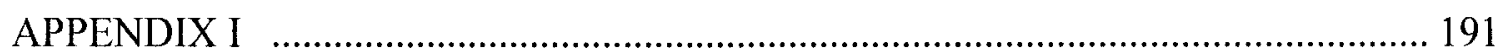

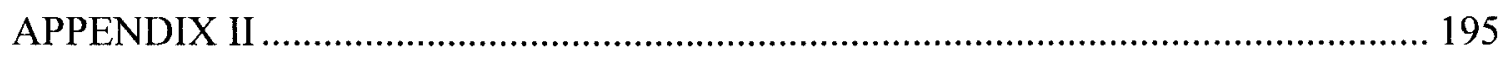

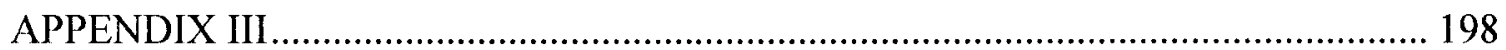

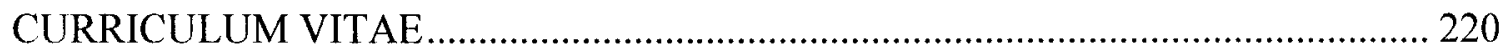




\section{LIST OF TABLES}

1-1. Noise methodologies or models till 2004

2-1. Data calibration values by site type; not including Site 10CA 10

3-1. Atmospheric attenuation per meter as a function of $1 / 3$-octave band center frequency for default atmospheric conditions

4-1. Ground Type and Effective Flow Resistivity 45

6-1. Measured and Predicted noise levels (dBA) at site $01 \mathrm{KY}, 02 \mathrm{KY}$ $03 \mathrm{KY}$, and $04 \mathrm{IN}$

6-2. Statistical analysis of errors at sites $01 \mathrm{KY}, 02 \mathrm{KY}, 03 \mathrm{KY}$, and $04 \mathrm{IN}$

6-3. Predicted insertion loss (dBA) by TNM 2.5 and STAMINA 2.0 at the site $01 \mathrm{KY}, 02 \mathrm{KY}$

6-4. Adjusted Insertion loss (dBA) by TNM 2.5 and STAMINA 2.0 at the site $01 \mathrm{KY}, 02 \mathrm{KY}$

7-1. Difference between mean measured and predicted sound levels at the ground effect dip for ground effect.

7-2. Two-tailed t-test for TNM and HNP at the ground effect dip for ground effect.

7-3. Descriptive statistics for comparison of predicted insertion loss at frequency dip by HNP and TNM

7-4. The predicted insertion loss (dBA) of a one-lane line source by TNM 2.5, STAMINA 2.0, and HNP 1.0 with varying distance. 
7-5. The predicted insertion loss (dBA) of a four-lane line sources by TNM 2.5, STAMINA 2.0, and HNP 1.0 with varying distance.

7-6. The comparison of statistical analysis of diffraction theories for case 1

7-7. The comparison of statistical analysis of diffraction theories for case 2

7-8. The comparison of statistical analysis of diffraction theories for case 3

7-9. The comparison of statistical analysis of diffraction theories for case 4

7-10. The comparison of statistical analysis of diffraction theories for case 5

7-11. The comparison of statistical analysis of diffraction theories for case 6

7-12. The comparison of statistical analysis of diffraction theories for case 7

7-13. The comparison of statistical analysis of diffraction theories for case 8

7-14. The comparison of statistical analysis of diffraction theories for case 9

7-15. The comparison of statistical analysis of diffraction theories for case 10

7-16. Comparison of absolute noise levels and insertion loss (dBA) of four propagation path model and two propagation path model by HNP with varying receiver distance at the height of $1.5 \mathrm{~m}(5 \mathrm{ft})$

7-17. Comparison of absolute noise levels and insertion loss (dBA) of four propagation path model and two propagation path model by HNP and TNM with varying receiver height at the distance of $15 \mathrm{~m}(50 \mathrm{ft})$ from the barrier

8-1. Comparisons of the absolute noise levels (dBA) by TNM, STAMINA, and HNP for site $01 \mathrm{KY}$

8-2. Statistical analysis for site $01 \mathrm{KY}$ 164

8-3. Comparisons of the insertion loss (dBA) by TNM, STAMINA, and HNP for the site $01 \mathrm{KY}$ 
8-4. Comparisons of predicted insertion loss by HNP 1.0 with 4 propagation path model and 2 propagation path model, with the measured insertion loss at the site $01 \mathrm{KY}$ 166

8-5. Comparisons of the absolute noise levels (dBA) by TNM, STAMINA, and HNP for the site 04IN 166

8-6. Comparisons of the absolute noise levels (dBA) by TNM, STAMINA, and HNP for the site 04CT. 167

8-7. Statistical analysis for the site $04 \mathrm{CT}$ 168

8-8. Comparisons of the insertion loss (dBA) by TNM, STAMINA, and $\mathrm{HNP}$ for the site $04 \mathrm{CT}$.

8-9. Comparisons of the absolute noise levels by TNM, STAMINA, and HNP for site $05 \mathrm{CT}$

8-11. Comparisons of the insertion loss (dBA) by TNM, STAMINA, and $\mathrm{HNP}$ for the site $05 \mathrm{CT}$.

8-12. Comparisons of the absolute noise levels (dBA) by TNM, STAMINA, and HNP for the site 08CA

8-13. Statistical analysis for the site $08 \mathrm{CA}$

8-14. Comparisons of the insertion loss (dBA) by TNM, STAMINA, and HNP for the site $08 \mathrm{CA}$

8-15. Comparisons of the absolute noise levels (dBA) by TNM, STAMINA, and HNP for the site 09CA

8-16. Statistical analysis for the site 09CA

8-17. Comparisons of the absolute noise levels (dBA) by TNM, STAMINA, and HNP for the site 11CA

8-18. Statistical analysis for the site 11CA. 
8-19. Comparisons of the insertion loss (dBA) by TNM, STAMINA, and HNP for the site 11CA

8-20. Comparisons of the absolute noise levels (dBA) by TNM, STAMINA, and HNP for the site 01MA

\section{APPENDIXES}

4-2. TNM's method to calculate $F(w)$

4-3. ACM 680 Algorithm to calculate $F(w)$

4-4. FFT method to calculate $F(w)$ 


\section{LIST OF FIGURES}

FIGURE

PAGE

3-1. National Reference Energy Mean Emission Levels as a Function of Speed 26

3-2. Reference Energy Mean Emission Levels as a function of speed (Fleming et al., 1995)

3-3. Air absorption attenuation as a function of distance ............................................. 32

4-1. Definition of specific acoustic impedance........................................................... 41

4-2. Normalized impedance value $(Z=R+i X)$ with $\sigma=200$ cgs Rayls .............. 43

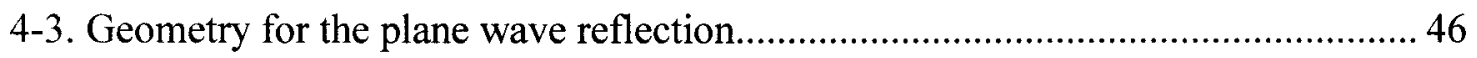

4-4. Source-receiver geometry for direct and reflected paths (De Jong, 1983)

4-5. Comparison TNM's method, ACM 680 Algorithm and

FFT method to calculate $F(w)$ 50

4-6. Difference between spherical and plane wave reflection coefficients 52

4-7. Set of predicted spectra for various values of flow resisitivity of ground surface

4-8. Schematic diagram for the different section of sound field

4-9. Real and imaginary parts of the Fresnel integral with $x$ between -10 and 10 56

4-10. Alternative plot for the Fresnel integral $F(x)$ 57

4-11. Diffraction of sound by semi-infinite plane for 
4-12. Geometry of diffraction at a hard wedge (De Jong, 1983) .................................. 60

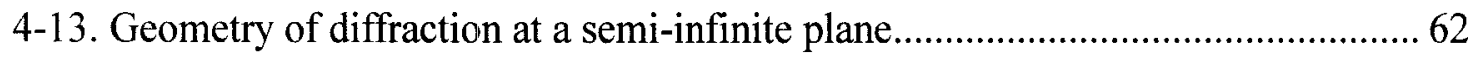

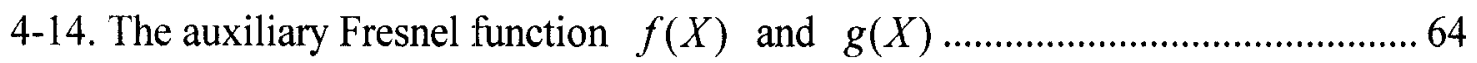

4-15. Geometry of diffraction at a screen (De Jong, 1983)....................................... 65

4-16. De Jong's model compared to ignoring the hard surface ...................................... 68

4-17. De Jong compared to ignoring the soft surface, and to the PROPAG from Delta (Ögren, 1997).

4-18. Definition of symbols for the Fresnel zone model (Ögren, 1997) 70

4-19. Fresnel-zone model compared to De Jong's model, distance $=100 \mathrm{~m}$, impedance discontinuity at $20 \mathrm{~m}, h_{r}=h_{s}=1 \mathrm{~m}$

4-20. Fresnel-Zone model compared to De Jong's model, distance $=100 \mathrm{~m}$, impedance discontinuity at $50 \mathrm{~m}, h_{r}=h_{s}=1.5 \mathrm{~m}$

4-21. Step-wise calculation of sound field (Rasmussen, 1982) 74

4-22. Comparison of the Kurze-Anderson's formula with experimental data $(\mathrm{Li}, 2005)$ 77

4-23. The four propagation paths for Janasson's model (De Jong, 1983)..................... 80

5-1. Reference microphone at measurement site $($ Lee, 1996)...................................... 88

7-1. Set of predicted spectra for various values of flow resistivity of the ground surface. Reference sound pressure level is that which would exist at the receiver in free field (Embleton, 1983)

7-2. Set of HNP predicted spectra for the same values of flow resistivity of the ground surface of Embleton's model

7-3. Comparison with the measurement by TNM and HNP over grassland, source receiver distance $35 \mathrm{~m}(114 \mathrm{ft})$

7-4. Comparison with the measurement by TNM and HNP 
over grassland, source receiver distance $62 \mathrm{~m}(202 \mathrm{ft})$

7-5. Comparison with the measurement by TNM and HNP over grassland, source receiver distance $110 \mathrm{~m}(360 \mathrm{ft})$

7-6. Comparison with the measurement by TNM and HNP over grassland, source receiver distance $195 \mathrm{~m}(640 \mathrm{ft})$

7-7. Comparison with the measurement by TNM and HNP over grassland, source receiver distance $348 \mathrm{~m}$ (1140 ft)

7-8. Best fit method for TNM, HNP and measurements at the ground effect dip for ground effect.

7-9. Comparison of barrier insertion loss in octave bands, source height $0.7 \mathrm{~m}$, receiver height $1.5 \mathrm{~m}$, barrier height $1.8 \mathrm{~m}$

7-10. Comparison of barrier insertion loss in octave bands, source height $0.7 \mathrm{~m}$, receiver height $1.5 \mathrm{~m}$, barrier height $4.9 \mathrm{~m}$

7-11. Comparison of barrier insertion loss in octave bands, source height $0.7 \mathrm{~m}$, receiver height $3 \mathrm{~m}$, barrier height $1.8 \mathrm{~m}$

7-12. Comparison of barrier insertion loss in octave bands, source height $0.7 \mathrm{~m}$, receiver height $3 \mathrm{~m}$, barrier height $4.9 \mathrm{~m}$

7-13. Comparison of barrier insertion loss in octave bands, source height $0.7 \mathrm{~m}$, receiver height $6 \mathrm{~m}$, barrier height $4.9 \mathrm{~m}$

7-14. Comparison of barrier insertion loss in octave bands, source height $0.7 \mathrm{~m}$, receiver height $12 \mathrm{~m}$, barrier height $1.8 \mathrm{~m}$

7-15. Comparison of barrier insertion loss in octave bands, source height $0.7 \mathrm{~m}$, receiver height $12 \mathrm{~m}$, barrier height $4.9 \mathrm{~m}$

7-16. Comparison of barrier insertion loss in octave bands, source height $0.7 \mathrm{~m}$, receiver height $6 \mathrm{~m}$, barrier height $1.8 \mathrm{~m}$

7-17. Comparison of predicted insertion loss with the measurements at minimum insertion loss by TNM and HNP

7-18. Plot for the predicted insertion loss of a one-lane line source by 
TNM 2.5, STAMINA 2.0, and HNP 1.0 with varying distance

7-19. Plot for the predicted insertion loss of a four-lane line sources by

TNM 2.5, STAMINA 2.0, and HNP 1.0 with varying distance.

7-20. Comparison of Maekawa's chart and MacDonald's solution, with $\mathrm{N}_{2}: \mathrm{N}_{1}=80: 1$

7-21. Comparison of Maekawa's chart and MacDonald's solution, with $\mathrm{N}_{2}: \mathrm{N}_{1}=10: 1$

7-22. Insertion loss comparison by different diffraction theories

7-23. Comparison of Kurze-Anderson and MacDonald diffraction theories for case 1 , source to barrier distance $5 \mathrm{~m}(16 \mathrm{ft})$

7-24. Comparison of Kurze-Anderson and MacDonald diffraction theory for case 2, source to barrier distance $20 \mathrm{~m}$ (66 ft)

7-25. Comparison of Kurze-Anderson and MacDonald diffraction theory for case 3, source to barrier distance $35 \mathrm{~m}$ (115 ft)

7-26. Comparison of Kurze-Anderson and MacDonald diffraction theory for case 4 , source to barrier distance $50 \mathrm{~m}(164 \mathrm{ft})$. 136

7-27. Comparison of Kurze-Anderson and MacDonald diffraction theory for case 5 , source to barrier distance $65 \mathrm{~m}(213 \mathrm{ft})$

7-28. Difference of insertion loss by MacDonald's curve and Kurze-Anderson's curve with varying source distances

7-29. Comparison of Kurze-Anderson and MacDonald diffraction theory for case 6 , receiver to barrier distance $5 \mathrm{~m}(16 \mathrm{ft})$

7-30. Comparison of Kurze-Anderson and MacDonald diffraction theory for case 7, receiver to barrier distance $20 \mathrm{~m}(66 \mathrm{ft})$

7-31. Comparison of Kurze-Anderson and MacDonald diffraction theory for case 8, receiver to barrier distance $35 \mathrm{~m}$ (115 ft).

7-32. Comparison of Kurze-Anderson and MacDonald diffraction theory for case 9 , receiver to barrier distance $50 \mathrm{~m}(164 \mathrm{ft})$ 
7-33. Comparison of Kurze-Anderson and MacDonald diffraction theory for case 10, receiver to barrier distance $65 \mathrm{~m} \mathrm{(213 \textrm {ft } )}$

7-34. Difference of insertion loss by MacDonald and Kurze-Anderson curve with varying receiver distances

7-35. Two propagation paths in TNM 146

7-36. Comparison of insertion loss by 4 propagation paths and 2 propagation paths for case 1 , receiver to barrier distance $15 \mathrm{~m}(50 \mathrm{ft})$ 148

7-37. Comparison of insertion loss by 4 propagation paths and 2 propagation paths for case 2 , receiver to barrier distance $30 \mathrm{~m}(100 \mathrm{ft})$ 150

7-38. Comparison of insertion loss by 4 propagation paths and 2 propagation paths for case 3 , receiver to barrier distance $60 \mathrm{~m}(197 \mathrm{ft})$ 150

7-39. Comparison of insertion loss by 4 propagation paths and 2 propagation paths for case 4 , receiver to barrier distance $120 \mathrm{~m}(394 \mathrm{ft})$

7-40. Comparison of insertion loss by 4 propagation paths and 2 propagation paths for case 5 , receiver to barrier distance $240 \mathrm{~m}(800 \mathrm{ft})$

7-41. Comparison of insertion loss by 4 propagation paths and 2 propagation paths for case 6 , receiver height $0 \mathrm{~m}(0 \mathrm{ft})$

7-42. Comparison of insertion loss by 4 propagation paths and 2 propagation paths for case 7 , receiver height $0.5 \mathrm{~m}(1.7 \mathrm{ft})$

7-43. Comparison of insertion loss by 4 propagation paths and 2 propagation paths for case 8 , receiver height $1.0 \mathrm{~m}(3.3 \mathrm{ft})$ 156

7-44. Comparison of insertion loss by 4 propagation paths and 2 propagation paths for case 9 , receiver height $2 \mathrm{~m}(6.6 \mathrm{ft})$

7-45. Comparison of insertion loss by 4 propagation paths and 2 propagation paths for case 10 , receiver height $4 \mathrm{~m}(13.2 \mathrm{ft})$ 158

7-46. Contour of insertion loss (dBA) of four propagation path model by HNP with varying receiver heights and distances 
7-47. Contour of insertion loss (dBA) of two propagation path model by HNP with varying receiver heights and distances

7-48. Contour of the difference of insertion loss (dBA) between four propagation path model and two propagation path model by HNP 161

8-20. Mean absolute noise levels with the barriers 176

8-21. Mean absolute noise levels without the barriers. 177

8-22. Insertion loss comparisons by TNM, STAMINA, and HNP 177

\section{APPENDIXES}

5-2. Graphic User Interface of HNP 1.0 for highway plan view. 196

5-3. Graphic User Interface of HNP 1.0 for multiple windows 197

6-1. Photograph for the field measurements at the barrier site $01 \mathrm{KY}$ 199

6-2. Photograph for the field measurements at the open site $04 \mathrm{IN}$ 200

8-1. Site 01KY: Description and Photograph 201

8-2. Site 01KY: TNM 2.5 Model Description, TNM Plan, Skew View 202

8-3. Site 02KY: Description and Photograph 203

8-4. Site 02KY: TNM 2.5 Model Description, TNM Plan, Skew View 204

8-5. Site 03KY: Description and Photograph 205

8-6. Site 04IN: Description and Photograph 206

8-7. Site 04IN: TNM 2.5 Model Description, TNM Plan, Skew View 207

8-8. Site 04CT: Description and Photograph 208

8-9. Site 04CT: TNM 2.5 Model Description, TNM Plan, Skew View and perspective view. 
8-11. Site 05CA: TNM 2.5 Model Description, TNM Plan, skew View, and perspective view.

8-12. Site 08CA: Description and Photograph

8-13. Site 08CA: TNM 2.5 Model Description, TNM Plan, skew View, and perspective view

8-14. Site 09CA: Description and Photograph 214

8-15. Site 09CA: TNM 2.5 Model Description, TNM Plan, Skew View, and Perspective View

8-16. Site 11CA: Description and Photograph 216

8-17. Site 11CA: TNM 2.5 Model Description, TNM Plan, Skew View, and Perspective View

8-18. Site 01MA: Description and Photograph 218

8-19. Site 01MA: TNM 2.5 Model Description, TNM Plan, Skew View, and Perspective View 


\section{CHAPTER I \\ INTRODUCTION}

\subsection{Background}

The mid-20th Century witnessed the peak construction period of the National Highway System (NHS). Cities were connected and goods and people were delivered to destinations in a fast, smooth and efficient way, which promoted economic and social development. These accomplishments of highway construction were based on the philosophy of economical benefit and engineering feasibility; however, environmental impacts were neglected (Cohn and McVoy, 1982). A series of problems related to the environment, including highway noise, air pollution and ecological deterioration, were coming into existence, which led to government concerns and public opposition. It was estimated that over ninety million people in this country were exposed to excessive highway noise (Harris, 1985). These environmental problems, together with a new thrust in environmental legislation, contributed to a new philosophy of transportation project planning concerning the social, economic and environment (SEE) effects.

According to Dr. Cohn, the evolutionary nature of transportation project development since 1960 may be viewed from three perspectives (Cohn and McVoy, 1982):

1. Legislation and regulations

2. Project development process 
3. Analytical modeling techniques

At the national level, environmental lobbies influenced Congress and the White House to enact a series of legislation regarding transportation and the environment. Among them, the Federal Aid Highway Act (FAHA) of 1962 and the National Environment Policy Act (NEPA) of 1969 were the most important legislation (Cohn and Harris, 1987).

The Federal Aid Highway Act (FAHA) of 1962 was the earliest legislation that put more emphasis on research and development and required the use of the 3-C planning

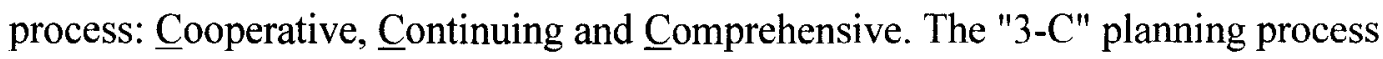
emphasized impact analysis and public involvement (Cohn and Harris, 1988).

Seven years later, the National Environmental Policy Act (NEPA) of 1969 was mandated, which was one of the nation's oldest, most important and far-reaching environmental laws (Bass et al., 2001). It required that Federal agencies prepare an Environment Impact Statement (EIS) for any major Federal action significantly affecting the environment.

NEPA established the general framework for decision making for all Federal agencies, while the Federal Aid Highway Act (FAHA) of 1970 set forth the specific mandates which required that an interdisciplinary approach to project development comply with the NEPA mandate (Cohn, 1982).

A substantial body of additional noise-related legislation also had been mandated in the same period. The House and Urban Development Act of 1965 was the earliest statutory reference for transportation noise. It required the determination of methods to 
minimize the economic impacts of airport noise. The Noise Control Act of 1972 gave considerable attention to transportation source noise control.

At the state level, the Federal Highway Administration (FHWA) issued FHPM 77-1 "Process Guidelines for the Development of Environmental Action Plans". This regulation provided the foundation for project development at the state level.

In 1982, the FHWA mandated a Federal-Aid policy "23 CFR 772" to provide procedures for noise studies and noise abatement measures to help protect the public health and welfare. It provided noise abatement criteria. The traffic noise analysis shall include the following five-step procedures under detailed study (Cohn and Harris, 1987):

1. Identification of existing activities, developed lands, and undeveloped lands for which development is planned, designed and programmed, which may be affected by noise from the highway

2. Prediction of traffic noise levels

3. Determination of existing noise levels

4. Determination of traffic noise impacts

5. Examination and evaluation of alternative noise abatement measures for reducing or eliminating the noise impacts

Among all the abatement measures, noise barriers are the most widely used and effective method to protect the surrounding community. Since the first noise barrier was built in 1963, more than 2,947 linear kilometers (1,831 linear miles) of noise barriers were built in the United States at a cost of more than $\$ 2.5$ billion as of 2001 (FHWA, 2002).

In order to evaluate the effectiveness of the proposed barrier, it is necessary to develop noise prediction methodologies and models for transportation noise analysis. In the United States, noise prediction methodologies and models have evolved from the late 
1960s since the first noise model, NCHRP-78, was developed, primarily through the funding efforts of the National Cooperative Highway Research Program (NCHRP) (Cohn and McVoy, 1982). Since then, many noise methodologies or models have been developed, as shown in Table 1-1.

Table 1-1. Noise methodologies or models till 2004

\begin{tabular}{ll}
\hline Date & Model \\
\hline 1965 & NCHRP 78, 1969 \\
1970 & NCHRP 117, 1971 \\
& TSC Model 1972 \\
& NCHRP 144, 1973 \\
1975 & NCHRP 173/174, 1976 \\
& FHWA Model, 1978 \\
& INM, 1978 \\
& SNAP and STAMINA, 1979 \\
& INM Version 2, 1979 \\
1980 & STAMINA Version 2, 1982 \\
1995 & TNM 1.0, 1998 \\
& TNM 1.0a,1999 \\
& TNM 1.0b,1999 \\
2000 & TNM 1.1, 2000 \\
& TNM 2.0, 2002 \\
& TNM 2.1 2003 \\
& TNM 2.5 2004 \\
\hline
\end{tabular}

Source: Cohn and Harris, 1999

The principal model used for highway noise analysis during the last 25 years was the FHWA model (FHWA-RD-77-108), which represented an achievement of more than a decade of research and development in highway noise prediction. The computer

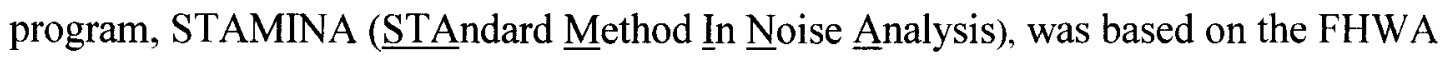
methodology. Since that time, there has been great advancement in the methodology for noise prediction, barrier analysis and design, and computer software development and hardware technology; therefore, the FHWA identified the need to design and develop a 
state-of-the-art highway traffic noise prediction model that utilized these advancements (Fleming et al., 1995). Under this circumstance, the FHWA Traffic Noise Model (TNM) model came into being. In March 1998, The FHWA TNM 1.0 was released. TNM has been updated several times and the latest version is TNM 2.5 .

As for engineering level modeling, TNM is a representation of highways, barriers and receivers for practical civil engineering applications. Therefore, model accuracy is the primary consideration (Sisti and Farr, 2000). How well it represents the real case depends on the accuracy compared to the field measurement data. Since the beginning release of STAMINA and TNM, many researchers have done studies to evaluate the model accuracy by comparing models to other models, and models to field measurement data. To increase the accuracy of STAMINA, much research was focused on Reference Energy Mean Emission Level (REMEL) data accuracy, and ground attenuation effects.

From the engineering perspective, increasing accuracy for noise prediction models can achieve the expected engineering satisfaction (minimizing highway noise) and reduce public opposition. From the economical perspective, improving model accuracy can provide barrier cost reduction and reduce negative visual impacts of barriers. Noise barriers are expensive to build, averaging $\$ 24,730$ per 1,000 square foot for concrete in the year of 2001 (FHWA, 2001); therefore, efficient, cost-effective highway noise barriers should be built. Nationwide, noise barriers average 3.6 meters (12 feet) high. A 1-decibel improvement in traffic noise prediction accuracy could reduce the barrier height by 0.6 meters ( 2 feet). A more accurate noise prediction model could have produced a cost savings of over $\$ 260,000,000$ for barriers already constructed (Kim, 2002). Therefore, increasing model accuracy is of great practical significance. 
Insertion loss is the most realistic measure to evaluate the effectiveness of noise barriers. Generally, $5 \mathrm{dBA}$ insertion loss should be achieved along the first row of receivers. Higher barriers will result in increased insertion loss; however, the construction cost will also increase. To make a balance between the benefit and cost of the barrier is very important.

Research has showed that TNM generally over-predicts insertion loss by several dBA compared with STAMINA. This suggests that TNM overstates the effectiveness of barriers and generates shorter barriers in height and length compared with STAMINA. If TNM predicts insertion loss in the same way as field measurements, it means a lot of money will be saved and an aesthetic design will be achieved. However, if TNM overpredicts the insertion loss compared with field measurements, it means TNM cannot achieve the expected effectiveness and the receivers cannot be fully protected by barriers with short heights.

This dissertation will make a comparative study of the physical mechanisms related to highway noise barrier insertion loss, and evaluate the accuracy of insertion loss of TNM 2.5 compared with STAMINA 2.0 and the field measurement data from the perspective of sound diffraction and propagation theories, and recommendations will be given on how to improve the accuracy of TNM's predictions of insertion loss. 


\subsection{Dissertation Organization}

Chapter I. Introduction

Chapter II. Problem Statement

Chapter III. Literature Review

Chapter IV. Theoretical Analysis

Chapter V. Methodology

Chapter VI. Field Measurements of Highway Barrier Insertion Loss

Chapter VII. Model Verification

Chapter VIII. Validation with the Field Measurements

Chapter IX. Conclusions and Recommendations 


\section{CHAPTER II \\ PROBLEM STATEMENT}

\subsection{Problem Definition}

Satisfactory performance of noise barriers depends on many aspects. From an acoustical perspective, factors involved in achieving desired performance include transmission loss, propagation loss, absolute sound levels with barriers, insertion loss and so on. However, insertion loss is the most important performance index; it is defined as the difference between acoustical levels before and after installation of a barrier, where the sound source, terrain, ground, and atmospheric conditions are equivalent (Lee and Fleming, 1996). Insertion loss is a catch-all measurement of the effectiveness of a sound barrier that has been "inserted" between a source and a receiver. It is the truest measure of the effusiveness of an installed barrier.

Noise barriers are usually designed with an insertion loss of $10 \mathrm{dBA}$. Actual barrier insertion loss of between 6 and $8 \mathrm{dBA}$ are quite common (Lee and Fleming, 1996). Since insertion loss is so important to barrier performance, the prediction of insertion loss by noise prediction models should be as accurate as possible. A prediction error for insertion loss within $1 \mathrm{dBA}$ may be tolerable for engineering and acoustical requirements; however, an error of more than $3 \mathrm{dBA}$ should be considered unacceptable.

For noise prediction models, insertion loss is calculated as the difference between absolute noise levels before and after a noise barrier is established. Therefore, insertion 
loss is composed of two parts: the absolute noise level at a receiver without a barrier and the absolute noise level at a receiver with a barrier. Error due to either part will contribute to the error of final insertion loss.

In March 1998, the FHWA released a newly developed computer program for highway noise prediction and analysis-Traffic Noise Model (TNM) version 1.0 (Anderson et al., 1998), to replace the old model STAMINA 2.0 (Bowlby et al., 1982). A FHWA memo (1998) which accompanied TNM release stated "The TNM has been validated and has been found to have improved accuracy over the existing FHWA prediction model." The memo also stated that the new model has been found to be from 1 to $6 \mathrm{dBA}$ more accurate than STAMINA, allowing for "more exactness in identification of highway traffic noise impacts." However, only two simple data sets (in California and Maryland) were provided, and these tests utilized 1974 REMEL data contained in the original release of STAMINA 2.0 (Bowlby et al., 1982).

Since 1998, there have been five additional releases: v1.0a, v1.0b, v1.1, v2.0 and v2.1; however, there was no indication of direct changes to the acoustical algorithms of the model (memo, 2004). A memo that accompanied the release of TNM 2.0 stated that "Comparing the field-measured data to the FHWA TNM-predicted data indicates that the model's sound propagation algorithms are functioning properly. This same comparison, however, indicates a general over-prediction in highway traffic noise levels that necessitates model calibration" (Memo, 2002). In April 2004, the FHWA TNM 2.5 was released; the memo stated that TNM 2.5 essentially eliminated vehicle emission level over-prediction and corrected a case-specific anomaly that was related to diffraction points. However, these statements were vague and they were not detailed enough to 
explain why these changes were made and how they were made from the technical perspective (Harris, 2004). It can be only inferred that except for one diffraction point anomaly, the whole diffraction and propagation theories employed in TNM were the same as the previous versions. In the newly released "TNM version 2.5 addendum to validation of FHWA's traffic noise model (TNM): phase 1 conducted by Volpe Center Acoustics Facility (VCAF)" (Rochat, 2004), VCAF stated that TNM v2.5 was performing extremely well for both uncalibrated and calibrated data, and the general over-prediction inherent in TNM 2.0, due to vehicle emissions or site-specific biases, is no longer an issue. Table 2-1 shows an overall result for all measurements by VCAF. Site 10CA was not included because VCAF reported that this site was an unusual site and it affected the barrier site average value. As can be seen, the average calibration decreases from $3.1 \mathrm{dBA}$ for TNM 2.0 to $0.5 \mathrm{dBA}$ for TNM 2.5 for all the site types. However, the average calibration for open area with soft ground still has a large calibration by $2.6 \mathrm{dBA}$ for TNM 2.5 .

Table 2-1. Data calibration values by site type; not including Site 10CA

\begin{tabular}{lcc}
\hline \multirow{2}{*}{ Site Type } & TNM v2.0 & TNM v2.5 \\
\cline { 2 - 3 } All & Average calibration (dBA) & Average calibration (dBA) \\
Open area, soft ground & 3.1 & 0.5 \\
Open area, hard ground & 4.2 & 2.6 \\
Barrier, soft ground & 4.1 & 1.1 \\
Ref mic in open & 2.1 & -0.8 \\
Ref mic above barrier & 4.1 & 1.8 \\
\end{tabular}

Source: TNM version 2.5 addendum to validation of FHWA's traffic noise model (TNM): phase 1 conducted by Volpe Center Acoustics Facility (VCAF) 
From this data, we can infer that specific characteristics in algorithms of TNM led to inaccuracy of the predicted noise levels. Corrections should be made in diffraction and propagation theories imbedded in the TNM model.

\subsection{Literature Review}

The earliest methodology for transportation noise prediction in this country was the National Cooperative Highway Research Program (NCHRP) Report 78 (Galloway et al., 1969); later studies included NCHRP report 117 (Gordon et al., 1971), NCHRP report 144 (Kugler and Piersol, 1973), and NCHRP Report 174 (Kugler et al., 1976). However, these methodologies were based on empirical equations, which applied to simple engineering situations. Given the fact that through 1980, more than 100 million dollars had been spent to construct more than 180 miles $(300 \mathrm{~km})$ of barriers throughout the U.S. (Cohn and Bowlby, 1984), it was necessary to develop a new noise prediction model. In response to this necessity, the FHWA developed a new noise prediction model named the "FHWA Traffic Noise Prediction Model", which ultimately became the computer program STAMINA 2.0 (Barry and Regan, 1978).

Since the early 1980's, users of STAMINA found that the STAMINA model would over-predict the absolute noise levels. Accordingly, research work began to enhance the prediction accuracy by comparing the field measurement data with STAMINA; much work was focused on REMEL data.

Harris (1984) demonstrated that at a $95 \%$ level of confidence, there was a significant difference between the national REMEL data and the field measurement data. This indicated that using the national REMEL data may lead to prediction inaccuracy. 
Hendriks (1985) found that the FHWA model over-predicted an average of 3.0 $\mathrm{dBA}$ to $4.0 \mathrm{dBA}$ over all vehicle categories, with measurements at 11 barrier sites in California. This suggested that the national REMEL data is not accurate enough to predict noise levels in California; new REMEL data should be developed for more accurate noise prediction.

Wayson (1993) demonstrated that the FHWA model tended to under-predict for automobiles and medium trucks and over-predict for heavy trucks. The study suggested that new state-specific REMEL data in Florida differed from the FHWA model.

A new four-state (Washington, Georgia, Colorado, and Kansas) REMEL data was developed, sponsored by the University of Louisville Research Foundation. Sheffer (1994) demonstrated that STAMINA, based on national REMEL, consistently over-predicted sound levels both with and without barrier situations compared to field measurement data. However, using a composite of the four-state REMEL data tended to lower the difference.

Some researchers suggested increasing REMEL data curves for vehicle types other than autos, medium trucks and heavy trucks for better reflection of the real characteristics of vehicle noise spectrum.

Cohn (1980) analyzed two situations through a series of noise measurements, and developed noise emission levels for a typical urban bus. He suggested that bus noise was more complex in its source components than those of autos and trucks, and should use different REMEL data.

Wayson (1993) also suggested that medium trucks should be categorized into at least two more vehicle classifications, given that medium trucks are only specified as a two-axle vehicle with six tires in the FHWA model. 
Some researchers studied ground attenuation in STAMINA, and gave suggestions for better accuracy from this perspective. Harris (1984) stated that the alpha factors ( 0 for hard ground, and 0.5 for soft ground) might be a possible source of problems for inexperienced users.

Hendriks (1995) conducted a total of 541 field measurements in order to study excess attenuation rates for traffic noise propagating over acoustically absorptive ground in terms of the alpha factor. He found that the present alpha scheme (0 for hard ground, 0.5 for absorptive ground) used in the FHWA Model caused average over-predictions of

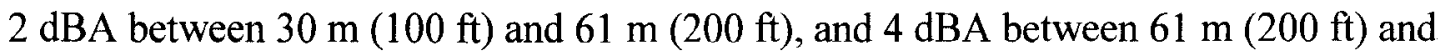
$122 \mathrm{~m}(400 \mathrm{ft})$. The study revealed that alpha factors were not only distance dependent, but also were height and vehicle type dependent.

Herman (1996) conducted a field measurement study with 41 sites along I-440 in Nashville; the result indicated that STAMINA 2.0 over-predicted the noise levels by 2.6 dBA. By using the empirically based ground attenuation algorithm-ORNAMENT, instead of using alpha factors directly, the over-prediction by STAMINA 2.0 decreased to $0.5 \mathrm{dBA}$, which was due solely to the ORNAMENT ground attenuation algorithm.

Since the release of TNM 1.0 in 1998, many researchers conducted a series of field measurements to validate model prediction accuracy by comparing both TNM and STAMINA to field measurement data.

Menge (1998) conducted TNM validation by comparing the TNM predicted insertion loss with the measured barrier insertion loss of Scholes (Scholes 1971) using the point source. The comparisons were made with a limited set of measured data with the following geometry: source height of $0.7 \mathrm{~m}(2.3 \mathrm{ft})$; barrier heights of $1.8 \mathrm{~m}(6 \mathrm{ft})$ and 4.9 
$\mathrm{m}(16 \mathrm{ft})$; microphone heights of $1.5 \mathrm{~m}(5 \mathrm{ft}), 3 \mathrm{~m}(10 \mathrm{ft}), 6 \mathrm{~m}(20 \mathrm{ft})$ and $12 \mathrm{~m}(40 \mathrm{ft})$,

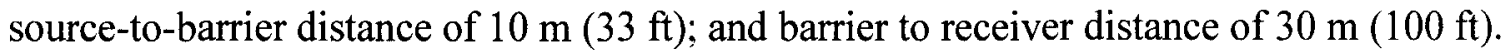
Comparisons of insertion loss with a point source showed that TNM were in agreement with field measurements. However, in some important frequency bands, TNM predicted questionable results compared with field data. In addition, only two simple cases (in California and Maryland) had been provided, both from Dec 9, 1997. A memo from the U.S Department of Transportation (USDOT, 1997) stated that TNM agreed well with the measured data, and STAMINA showed less agreement with the same measurements. However, STAMINA utilized old 1974 REMEL data in the tests (Harris, 2000). Harris (1998) conducted a project in Kansas that provided a chance to evaluate the performance of TNM versus of the performance of a version of STAMINA that utilized Kansas specific vehicle noise emission data (REMEL's). The conclusions were that STAMINA generated results that were consistent and in reasonably close agreement with measured data at the same location ( $0.5 \mathrm{dBA}$ under-predictions to $2.3 \mathrm{dBA}$ overpredictions), while TNM generated results that were less accurate than the STAMINA results at the same location ( $2.5 \mathrm{dBA}$ under-predictions to $4.1 \mathrm{dBA}$ over-predictions). Furthermore, observations were found that TNM results were very inconsistent, showing significant decreases in sound levels (1.6 dBA) when adding one lane of pavement to the existing condition, and significant increases in sound levels $(2.1 \mathrm{dBA})$ by deleting one lane.

Harris (2000) conducted a study to compare the accuracy of TNM and STAMINA in the Atlanta metropolitan area. Three sites with barriers and three sites without barriers were selected and accurately built into both models. Based on the comparison between 
measured and predicted sound levels, Harris stated that STAMINA, with GDOT's REMEL data, was more accurate than the TNM model, which over-predicted the absolute sound levels by an average of $3.2 \mathrm{dBA}$. For situations with a barrier, the results showed that STAMINA proved to be more accurate than TNM when predicting insertion loss, and TNM tended to over-predict the insertion loss by $1.2 \mathrm{dBA}$.

Cohn (2001) conducted a study to determine if Arizona state REMEL data was statistically different from the national REMEL data used in the FHWA TNM 1.0 model. Cohn compared the difference of predicted and measured noise levels using four models, which are STAMINA with 1974 national REMELs, STAMINA with the 1998 Arizona REMELs, TNM with 1995 National REMELs, and TNM with the 2000 Arizona REMELs. Cohn found that the use of Arizona state-specific REMEL data increased the accuracy of both STAMINA and TNM over national REMEL data. Results indicated that over-predictions by STAMINA 2.0 decreased from an average 3.2 to $1.1 \mathrm{dBA}$. The overpredictions by TNM decreased from an average 4.3 to $3.0 \mathrm{dBA}$. Cohn concluded that STAMINA was more accurate than TNM, and STAMINA with Arizona state-specific

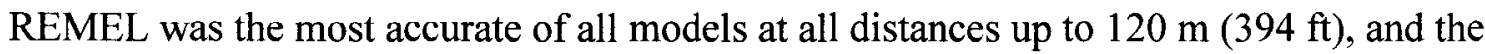
TNM model significantly over-predicted sound levels in this study. This implied that some other factors other than REMEL data influenced TNM accuracy and contributed to this $3.0 \mathrm{dBA}$ over-predictions.

Barrett et al. (2001) conducted the evaluation of nine highway noise barriers in New York State with both STAMINA 2.0 and TNM 1.0 and compared the results of two models. They concluded that in the "no barrier" cases, the noise level differences between the two models were small (on average, within about 1 to $2 \mathrm{dBA}$ ). But in all "with 
barrier" situations, TNM 1.0 predicted average greater insertion loss ( 1 to $3 \mathrm{dBA}$ ) than STAMINA did, with the noise reduction about 6 to $8 \mathrm{dBA}$ for TNM, and 5 to $7 \mathrm{dBA}$ for STAMINA. Barrett explained that two reasons accounted for this greater insertion loss. One was that STAMINA did not take any ground effects into account in "with barrier" cases, while TNM accounted for ground effects in all cases. Another reason was that a difference existed in sub-source height and their energy distributions to lower and upper sub-sources in TNM. As to the costs of mitigation, the study indicated that TNM generated greater insertion loss than STAMINA with the same barrier height, which resulted in lower mitigation costs by recommendation of lower barriers.

From July 1999 to August 2002, in support of the FHWA and the California Department of Transportation (Caltrans), the Volpe Center Acoustics Facility (VCAF) conducted a study to quantify and assess the accuracy of TNM (Rochat et al., 2002). Seventeen sites with barriers or without barriers were selected and modeled with TNM. The comparison between predicted values and measured values were evaluated. The results applied to TNM 2.0 since there were only minor changes in acoustic algorithm (0.1 to $0.2 \mathrm{dBA}$ ) for version 1.0. Overall, results showed that there was a $2.6 \mathrm{dBA}$ overprediction based on directly comparing uncalibrated TNM-predicted and measured sound levels. This over-prediction was due to vehicle emissions or site-specific bias. After calibration for the data, the over-prediction reduced to $1.0 \mathrm{dBA}$ for all-wind data.

In 2002, Hankered Environmental, Inc (Hankard, 2002) conducted a study to evaluate which model (STAMINA 2.0 or TNM 1.1) was better to predict the sound levels behind barriers, compared to the field measurement data for preparing I-25 Corridor Environment Assessment (EA). The prediction for STAMINA 2.1 ranged from $5 \mathrm{dBA}$ 
below and $8 \mathrm{dBA}$ above measured data. The average value was $1 \mathrm{dBA}$ above measured level from 75 measurements, and $73 \%$ (55 out of 75 ) of all predicted values fell within -3 to $+3 \mathrm{dBA}$. On the other hand, the prediction for TNM 1.1 ranged from $3 \mathrm{dBA}$ below, and $10 \mathrm{dBA}$ above the measured data, with averages $4 \mathrm{dBA}$ above the measured levels, and $36 \%$ percent ( 27 out of 75 ) of the predicted values fell within -3 to $+3 \mathrm{dBA}$. Overall, the study showed that TNM 1.1 predicted 2 to $5 \mathrm{dBA}$ more insertion loss than STAMINA did; the insertion loss unexpectedly increased after $213 \mathrm{~m}$ (700 ft) with TNM, but not with STAMINA. The study concluded that STAMINA 2.0 provided reasonably accurate and expected results in this case and TNM provided less accurate results.

In Florida, the effectiveness of barriers was investigated with TNM $1.0 \mathrm{~b}$ and STAMINA (Wayson et al., 2002); noise levels of 20 in-situ barriers were measured in phase 1 in three years and resulted in 844 discrete, $20 \mathrm{~min} L_{e q}$ data points. Barrier insertion loss was calculated according to the ANSI indirect barrier method. The findings for phase 1 indicated that TNM often, but not always, predicted greater insertion losses than STAMINA 2.0 or 2.1. STAMINA predicted better reference energy mean emission levels while TNM predicted better propagation losses. The author believed that it was because TNM continued to account for the ground effects in presence of a tall noise barrier, which STAMINA did not. In phase 2, seven more barriers were visited. As to the absolute predicted values, TNM performed the best in five out of six statistical categories evaluated, with an average error of $2.8 \mathrm{dBA}$ for TNM, $4.0 \mathrm{dBA}$ for STAMINA, and 3.3 dBA for STAMINA 2.1. As to the propagation loss, TNM outperformed STAMINA in all statistical categories evaluated except for maximum error. A $-4.1 \mathrm{dBA}$ average error indicated that STAMINA under-predicted propagation loss, compared with the measured 
data. By using the indirect insertion loss measurements, the predicted insertion loss could be compared with the measured insertion loss. The results showed that TNM predicted greater insertion loss without adjustment (2.1 to $3.7 \mathrm{dBA})$ at two sites and also predicted greater adjusted insertion loss (1.1 to $2.5 \mathrm{dBA})$.

In 2003, Wayson et al. (2003) conducted a continuation work of the previous Florida Department of Transportation (FDOT) study. Their investigation was evaluated on spectrum differences of TNM predicted and measured ground effects by analysis of in-situ measurements for 19 noise barriers. The results showed that TNM over-predicted sound levels, on average, by about $1 \mathrm{dBA}$ for microphone positions one and four, which were $1.5 \mathrm{~m}(5 \mathrm{ft})$ high, $15 \mathrm{~m}(50 \mathrm{ft})$ far and $1.5 \mathrm{~m}(5 \mathrm{ft})$ high, $30 \mathrm{~m}(100 \mathrm{ft})$ far from the barrier respectively.

Staiano (2003) examined relatively distant receivers and extreme topography in the entirely wooded areas with TNM. The prediction under-estimated the measured sound levels by 6 to $12 \mathrm{dBA}$. A series of parameters in TNM were evaluated to improve the agreement with measurements. Using ground impedance (forest floor, pine or hemlock, 20 to 80 Rayls) and grass did not help and TNM also showed a slight tendency to increase the excess attenuation with decreasing impedance, which did not comply with the expected results. The best result was achieved by using a tree zone, with a "field grass" default surface and additional terrain lines, with $2 \mathrm{dBA}$ average excess attenuation. In the study, excess attenuation was defined as the difference between predicted sound level (relative to the reference) and that of measured data.

Kim (2003) examined noise barrier designs in six different states (Arizona, Georgia, Kansas, Indiana, Louisiana, and Missouri) with TNM and STAMINA to make a 
direct comparison between absolute noise levels and insertion loss. The result showed that the average absolute noise level difference was within -1.0 to $+1.0 \mathrm{dBA}$ between STAMINA and TNM. However, TNM predicted about $3 \mathrm{dBA}$ greater insertion loss than STAMINA.

\subsection{Research Objectives}

Research studies in the previous section clearly indicate that the accuracy of TNM was questioned right after its release. These studies suggested a statistical difference of absolute sound levels with and without noise barriers between STAMINA 2.0 and TNM 2.5 and a difference of absolute sound levels with and without noise barrier between TNM 2.5 and field measurement data. Also, studies suggested that TNM over-predicted the insertion loss compared to STAMINA 2.0 and field measurement data.

Much work has been done on the improvement of model accuracy by identifying and characterizing the differences between national REMEL data and state-specific REMEL data. Investigations indicated that REMEL data had changed during the last 20 years, and state-specific REMEL data was more accurate than national REMEL data.

There are fewer studies on the identifying and characterizing of over-prediction of insertion loss from the perspective of diffraction and propagation algorithms of TNM. They are probably due to the following reasons:

1. TNM 2.5 uses completely different diffraction and propagation theories compared with STAMINA 2.0. Sound level calculations are based on the frequency spectrum, unlike STAMINA 2.0, which uses a dominant frequency of $550 \mathrm{~Hz}$. This provided additional complexity for the research. 
2. By using the ANSI indirect method to calculate barrier insertion loss, errors can be reduced with the help of reference receivers. However, this method is a compromise, since errors of insertion loss can be reduced for specific receiver points. On the other hand, some errors of insertion loss can be induced for other receiver points, because the ANSI indirect method is a linear method to compensate for insertion loss errors. However, the predicted sound field behind a noise barrier is a nonlinear phenomenon with complex physical interaction of sound waves and ground cover. Later work will demonstrate this point of view.

3. Without the source code of TNM 2.5, it is hard to study the diffraction and propagation algorithms in TNM 2.5 .

The basic objective of this research is to make a comparative study of the physical mechanisms related to highway noise barrier insertion loss, and evaluate the accuracy of insertion loss of TNM 2.5 compared with STAMINA 2.0 and the field measurement data from the perspective of sound diffraction and propagation theories, and recommendations will be given on how to improve the accuracy of TNM's predictions of insertion loss. In order to focus on the main objective of this research, a new model, HNP 1.0 (Highway Noise Predictor V1.0), was developed to compare to TNM 2.5, STAMINA 2.0 and the field measurement data. The following parameters will be used in the new model:

1. National REMEL data field measurements of FHWA/TSC (TSC: Transportation Systems Center), in California (CA), Florida (FL), Maryland (MD), Massachusetts (MA), Michigan (MI), New Jersey (NJ), and Tennessee (TN) during the period from July 1993 to November 1995 (Fleming et al., 1995). 
2. The same sub-source height as TNM 2.5 that is lower source height of $0 \mathrm{~m}(0 \mathrm{ft})$, upper source height of $1.5 \mathrm{~m}(5 \mathrm{ft})$ for autos and medium trucks, and lower source height of $0 \mathrm{~m}(0 \mathrm{ft})$, upper source height of $3.66 \mathrm{~m}(12 \mathrm{ft})$ for heavy trucks.

3. Average pavement will be used in both TNM 2.5 and HNP 1.0 due to the requirement of FHWA.

4. Neutral weather conditions will be used in both TNM 2.5 and HNP 1.0.

This study will be conducted with the following objectives in mind:

1. Comparing the frequency spectrum of reflection theory of a point source of HNP 1.0 with that of TNM 2.5 and the measurements by Parkin and Scholes (Parkin, 1965)

2. Comparing the frequency spectrum of insertion loss of a point source of HNP 1.0 with that of TNM 2.5 and the measurements by Scholes (Scholes, 1971)

3. Comparing the insertion loss at receiver points by a line source of HNP 1.0 with that of TNM 2.5 and STAMINA 2.0

4. Comparing the insertion loss at receiver points by multiple line sources of HNP 1.0 with that of TNM 2.5 and STAMINA 2.0

5. Comparing the insertion loss at receiver points of HNP 1.0 with TNM 2.5, STAMINA 2.0, and the field measurements on I-264

6. Comparing the absolute sound levels at receiver points of HNP 1.0 with TNM 2.5 , STAMINA 2.0, and the field measurements data on Road 111, IN

7. Comparing the insertion loss at receiver points of HNP 1.0 with Volpe Validation data 
8. Making recommendations for applications of the results of this research and further work

Based on the above analysis, conclusions and recommendations will be made as to how the results of this research can be applied to the highway noise barrier projects in the United States.

\subsection{Potential Contributions of this Research}

To date there have been no independent efforts to examine this issue in great detail. The reasons that TNM 2.5 over-predicts the insertion loss from the perspective of noise diffraction and propagation theories need to be identified, and this proposed research represents the first efforts to do just that. Corrections can be made for more accurate prediction of the insertion loss if it can be proved that the over-prediction of insertion loss by TNM 2.5 is partly caused by the diffraction and propagation theories. Since noise barriers are expensive to build, more accurate prediction of insertion loss by $1 \mathrm{dBA}$, not only means people can be protected by noise barriers with expected satisfaction, but also a significant cost saving potential can be achieved.

State highway agencies should provide noise barriers with an efficient costeffectiveness ratio. To achieve this goal, accurate noise prediction models are the fundamental requirements. New software HNP 1.0 was developed for this purpose, which predicts more accurate insertion loss for highway noise barrier. This research has the potential to make a significant contribution to this effort. 


\section{CHAPTER III}

\section{LITERATURE REVIEW}

\subsection{Noise Models}

\subsubsection{STAMINA 2.0}

STAMINA 2.0 is based on the FHWA Highway Traffic Noise Prediction Model (Report No. FHWA-RD-77-108). Just as for the other models, FHWA STAMINA 2.0 predicts a noise level through a series of adjustments to a reference sound level. In the FHWA model, the reference level is the energy mean emission level (REMEL), which is the empirical foundation of the prediction model. Adjustments are then added to the reference energy mean emission level to account for traffic flows, for varying distances from the roadway, for finite length roadways, and for shielding. This relation is given by the following equation:

$$
\begin{array}{rlr}
L_{e q}(h)_{i} & =\left(\bar{L}_{o}\right)_{E_{i}} \text { Reference energy mean emission level } \\
& +10 \log \left(\frac{N_{i} \pi D_{o}}{S_{i} T}\right) \quad \text { traffic flow adjustment } \\
& +10 \log \left(\frac{D_{o}}{D}\right)^{1+\alpha} & \text { distance adjustment } \\
& +10 \log \left(\frac{\psi_{\alpha}\left(\phi_{1}, \phi_{2}\right)}{\pi}\right) & \text { finite roadway adjustment } \\
& +\Delta_{S} & \text { shielding adjustment }
\end{array}
$$


where,

$L_{e q}(h)_{i} \quad$ the hourly equivalent sound level of the $\mathrm{i}^{\text {th }}$ class of vehicles

$\left(\bar{L}_{O}\right)_{l: i} \quad$ the reference energy mean emission level of the $\mathrm{i}^{\text {th }}$ class of vehicles

$N_{i} \quad$ the number of vehicles in the $\mathrm{i}^{\text {th }}$ class passing a specified point during some specified time period ( 1 hour)

$D$ the perpendicular distance, in meters, from the centerline of the traffic lane to the observer

$D_{0} \quad$ the reference distance at which the emission levels are measured, in the FHWA model is equal to 15 meters

$S_{i} \quad$ the average speed of the $\mathrm{i}^{\text {th }}$ class of vehicles and is measured in kilometers per hour $(\mathrm{km} / \mathrm{h})$

$T$ the time period over which the equivalent sound level is computed (1 hour)

$\alpha \quad$ a site parameter whose values depend upon site conditions

$\psi \quad$ a symbol representing a function used for segment adjustments, i.e., an adjustment for finite length roadways

$\phi_{1}, \phi_{2} \quad$ roadway angles of acoustic influence which assist in locating the roadway spatially

$\Delta_{S} \quad$ the attenuation, in dBA, provided by some type of shielding such as barriers, rows of houses, densely wooded areas, etc.

The first two lines of Equation (3-1) predict the equivalent sound level generated by a flow of vehicles of a single class traveling at a constant speed on an effectively infinite, flat roadway at a reference distance of 15 meters ( 50 feet). The last three lines of Equation (3-1) represent adjustments that deal with the site conditions between the observer and the roadway. 
Once $L_{e q}(h)_{i}$ is calculated, the total hourly equivalent sound level, $L_{e q}(h)$, can be determined. The $L_{e q}(h)$ is the sum of the acoustic contributions of the various classes of vehicles on that roadway. In the FHWA model, there are three classes of vehicles: automobiles (A), medium trucks (MT), and heavy trucks (HT). The total hourly equivalent sound level is computed as:

$$
L_{e q}(h)=10 \log \left(10^{\frac{L_{e q}(h)_{t}}{10}}+10^{\frac{L_{e q}(h)_{M T}}{10}}+10^{\frac{L_{e q}(h)_{I I T}}{10}}\right)
$$

\subsubsection{Reference Energy Mean Emission Level (REMEL)}

The first step in the prediction procedure is to determine the reference energy mean emission level (REMEL) for each class of vehicles that uses the highway. This requires the knowledge of the emission levels of the individual vehicles traveling on the highway. The emission level, $L_{o}$, is defined as the A-weighted peak pass-by noise level generated by a vehicle as measured by a microphone at a specified location. Emission levels depend on several factors, such as the type of vehicle, engine size, speed, tire type, etc. Based on the standard curve fitting and statistical techniques, STAMINA uses the following $\mathrm{A}$-weighted national reference energy mean emission levels:

$$
\begin{aligned}
& \left(\bar{L}_{o}\right)_{E_{A}}=38.1 \log (S)-2.4 \\
& \left(\bar{L}_{o}\right)_{E_{M T}}=33.9 \log (S)+16.4 \\
& \left(\bar{L}_{o}\right)_{E_{H T}}=24.6 \log (S)+38.5
\end{aligned}
$$

where,

S the average vehicle speed of the vehicle class in $\mathrm{km} / \mathrm{h}$ 
The reference energy mean emission levels shown here are plotted in Figure 3-1. The truck levels are national averages based on the truck data acquired in the report, Highway Noise Measurements for Verification of Prediction Models (FHWA, 1978).

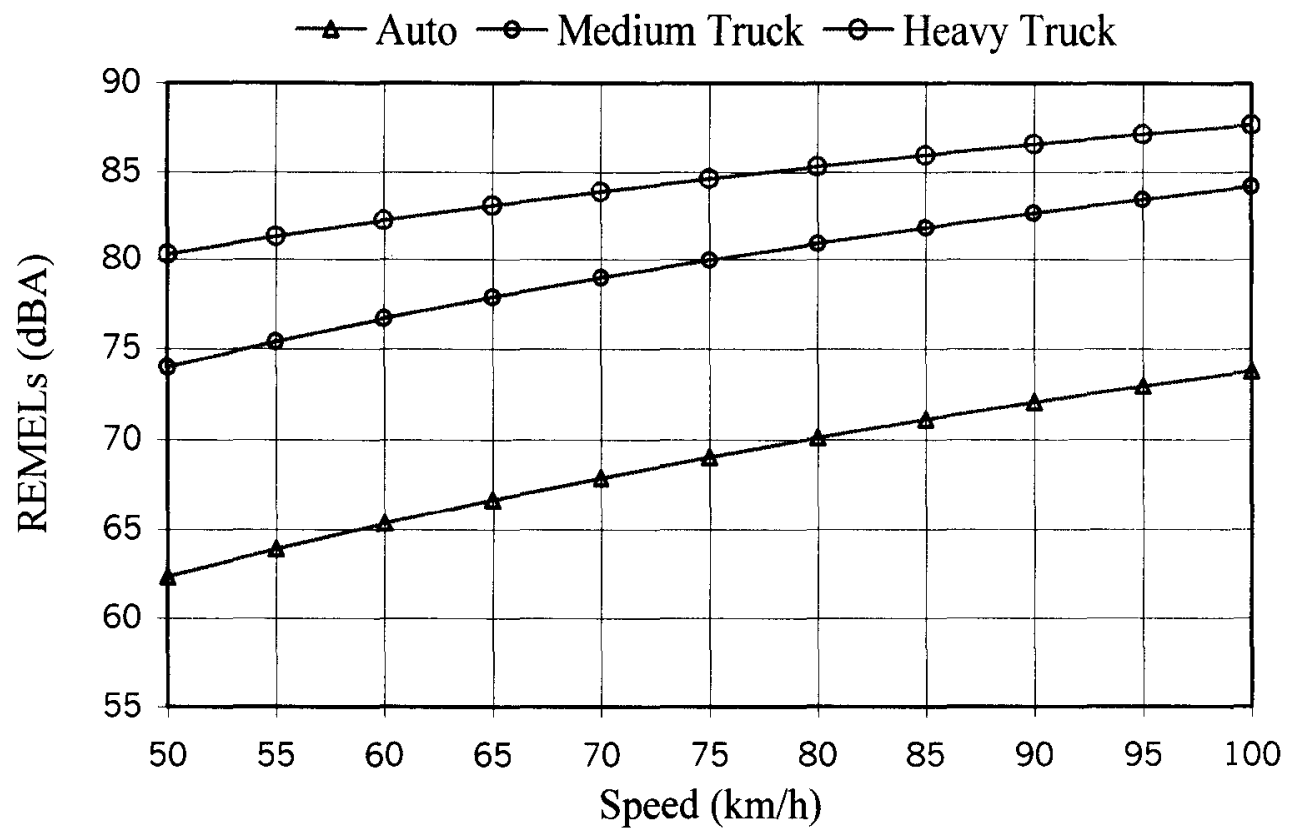

O SOURCE: "Statistical Analysis of FHWA Traffic Noise Data," FHWA-RD-78-64

$\triangle$ SOURCE: "Update of TSC Highway Traffic Noise Prediction Code (1974)," FHWA-RD-77-19

Figure 3-1. National Reference Energy Mean Emission Levels as a Function of Speed

\subsubsection{TNM 2.5}

TNM 2.5 computes highway traffic noise at receivers to help the design of highway noise barriers. It includes new REMEL data developed during 1994-1995 for the following five cruise-throttle vehicles types (shown in Figure 3-2); only the first three types are included in STAMINA 2.0.

- Automobiles

- Medium trucks

- Heavy trucks

- Buses 
- Motorcycles

According to the TNM Technical Manual (Menge et al., 1998), TNM takes the whole frequency spectrum into account and determines each vehicle type's total measured noise emissions as follows:

$$
\begin{gathered}
E_{A}\left(s_{i}\right)=\left(0.6214 s_{i}\right)^{A / 10} 10^{B / 10}+10^{C / 10} \\
L_{\text {emis, },}\left(s_{i}, f\right)=10 \times \log _{10}\left(E_{A}\right)+\left(D_{1}+0.6214 D_{2} s_{i}\right)+\left(E_{1}+0.6214 E_{2} s_{i}\right)\left[\log _{10} f\right] \\
+\left(F_{1}+0.6214 F_{2} s_{i}\right)\left[\log _{10} f\right]^{2}+\left(G_{1}+0.6214 G_{2} s_{i}\right)\left[\log _{10} f\right]^{3} \\
+\left(H_{1}+0.6214 H_{2} s_{i}\right)\left[\log _{10} f\right]^{4}+\left(I_{1}+0.6214 I_{2} s_{i}\right)\left[\log _{10} f\right]^{5} \\
+\left(J_{1}+0.6214 J_{2} s_{i}\right)\left[\log _{10} f\right]^{6}
\end{gathered}
$$

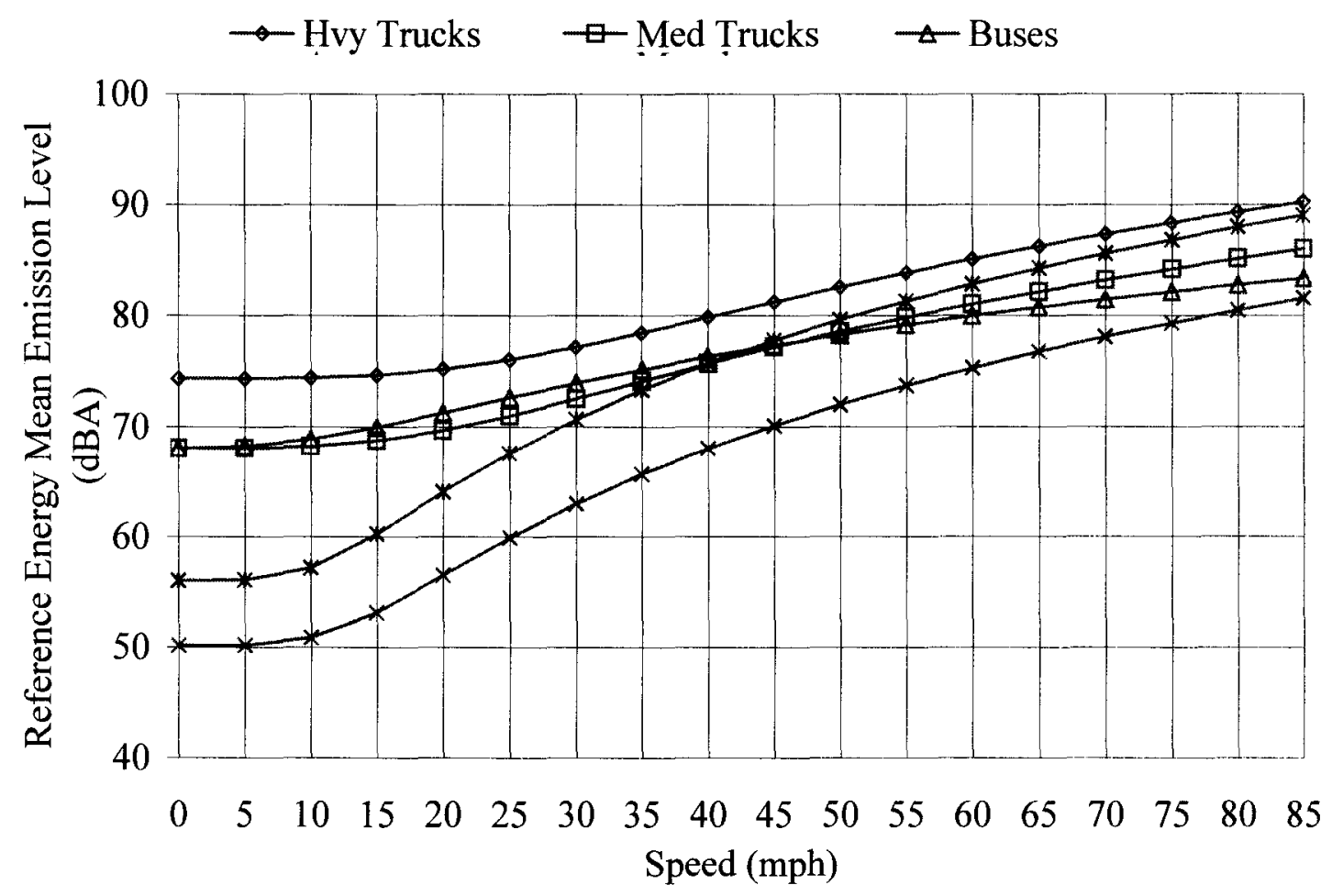

Figure 3-2. Reference Energy Mean Emission Levels as a function of speed (Fleming et al., 1995) 
Noise emission levels consist of A-weighted sound levels, 1/3-octave band spectra, and sub-source height strengths for the following pavement types:

- Dense-graded asphaltic concrete (DGAC)

- Portland cement concrete (PCC)

- Open-graded asphaltic concrete (OGAC)

- A composite pavement type consisting of data for DGAC and PCC combined TNM 2.5 calculates sound energy propagation, in 1/3-octave bands, between highway sources and nearby receivers. TNM 2.5 takes the following factors into account:

- Atmospheric absorption

- Divergence

- Intervening ground: its acoustical characteristics and its topography

- Intervening barriers: walls, berms and their combination

- Intervening rows of buildings

- Intervening areas of heavy vegetation

Like STAMINA 2.0, TNM 2.5 computes predicted noise levels through a series of adjustments to the REMEL data. Adjustments are then made to the emission levels to account for traffic flow, distance, and shielding. These factors are related by the following equation:

$$
L_{\text {Aeqlh }}=E L_{i}+A_{\text {traff }(i)}+A_{d}+A_{s}
$$

where,

$E L_{i} \quad$ the vehicle noise emission level for the $i^{\text {th }}$ vehicle type

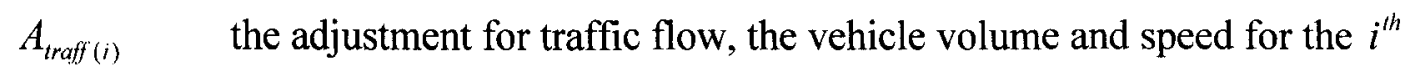
vehicle type 
$A_{d} \quad$ the adjustment for distance between the roadway and receiver and for the length of the roadway

$A_{s} \quad$ the adjustment for all shielding and ground effects between the roadway and the receiver. The TNM is based on a three-dimensional coordinate system.

\subsubsection{Traffic Flow Adiustment}

The adjustment for traffic flow is a function of vehicle volume and speed. The adjustment is computed separately for each vehicle type:

$$
A_{\text {traff }(i)}=10 \times \log _{10}\left(\frac{V_{i}}{S_{i}}\right)-13.2 \mathrm{~dB}
$$

where,

$V_{i} \quad$ the vehicle volume in vehicles per hour

$S_{i} \quad$ the vehicle speed in kilometers per hour

\subsubsection{Distance and Roadway Length Adjustment}

The adjustment for distance from the elemental roadway segment to the receiver and for the length of the segment is given by the following:

$$
A_{d}=10 \times \log _{10}\left[\left(\frac{15}{d}\right)\left(\frac{\alpha}{180}\right)\right] \mathrm{dB}
$$

where,

$d \quad$ the perpendicular distance to the line representing the roadway segment in meters

$\alpha \quad$ the angle subtended by the elemental roadway segment in degrees

TNM defines source-to-receiver elemental triangles by the closest angular spacing, at the receiver, of all object endpoints in the XY plane. When the three points of the elemental triangle are collinear ( $d=0$ and $\alpha=0$ ) or nearly collinear, TNM 2.5 uses a 
different equation for such cases. The equation is based on the distances from the receiver to the two endpoints of the elemental roadway segment $\left(d_{1}\right.$ and $\left.d_{2}\right)$ as follows:

$$
A_{d}=10 \times \log _{10}\left[\frac{\left|d_{2}-d_{1}\right|}{d_{2} d_{1}}\right]+12 \mathrm{~dB}
$$

\subsubsection{Vertical Geometry Acoustics}

TNM 2.5 uses the acoustical propagation algorithm to compute $A_{s}$, the adjustment for ground effects and shielding. All references are to the two-dimensional vertical plane, and all of the computations are based on point-source mathematics.

\subsubsection{Basis of the Acoustical Model}

The acoustical algorithms of TNM 2.5 for determining the effects of ground and shielding from barriers are based on sound propagation research from the previous 30 years. TNM 2.5 adopted the reflection theory of Chessell (1977) to calculate the effects of ground. His work expanded on previous studies, including Delany and Bazley (1970), and Embleton, et al. (1983). TNM 2.5 incorporates selected ground types with values of EFR based on their measurements.

The diffraction model is based on De Jong's model (De Jong, 1983). De Jong's diffraction theory is based on MacDonald's analytical solution. His solution works well for a limited number of reflections and diffractions in series, but it can't correctly compute the large number of components that could make up typical highway crosssection geometry. For these more complex geometries, the TNM 2.5 creates a straightline regression fit to the (two-dimensional) ground and averages the ground impedance in the vicinity of the reflection point using the approach of Boulanger (1997). This method 
has been validated through scale-model measurements, and agrees well with the De Jong's model for simpler cases, where the De Jong's model performs properly.

\subsection{Sound Absorption}

The atmosphere influences sound propagation via air absorption, refraction by wind and temperature conditions, and scattering by turbulence. Air absorption can be regarded as a property of the medium, which is a function of frequency for a given set of temperature and humidity values. Air absorption can easily be applied to a noise prediction model with lookup tables from a practical point of view. Refraction and turbulence are difficult to represent mathematically and they are not considered in either TNM 2.5 or STAMINA 2.0 .

TNM uses 1993 ISO standard (ISO 9613-1) to compute atmospheric absorption. The default temperature is $20^{\circ}$ Celsius ( $68^{\circ}$ Fahrenheit, 293.15 Kelvin) and the default humidity is 50 -percent relative humidity $(\mathrm{RH})$. Atmospheric attenuation per meter as a function of 1/3-octave band center frequency for default atmospheric conditions are given in Table 3-1.

Figure 3-3 shows air absorption attenuation as a function of distance. As can be seen, the attenuation due to air absorption gets larger and larger, especially in high frequencies when the receiver is located at further distances. For example, at the distance of $305 \mathrm{~m}(1,000 \mathrm{ft})$ from the source, there is about $6 \mathrm{dBA}$ noise attenuation due to air absorption for the frequency of $3,000 \mathrm{~Hz}$. However, we know that for most vehicles, especially for heavy trucks, low and middle frequencies dominate the noise spectrum. Noise attenuation due to air absorption is limited. Noise levels remain high. To reduce the 
noise levels to an acceptable level, many techniques including noise barriers should be assessed and effectively applied.

Table 3-1.

Atmospheric attenuation per meter as a function of $1 / 3$-octave band center frequency for default atmospheric conditions

\begin{tabular}{cccc}
\hline $\begin{array}{c}\text { 1/3-Octave Band } \\
\text { Center Freq..Hz }\end{array}$ & $\begin{array}{c}\text { Atten. } \\
\text { (dBA/m) }\end{array}$ & $\begin{array}{c}\text { 1/3-Octave Band } \\
\text { Center Freq.,Hz }\end{array}$ & $\begin{array}{c}\text { Atten. } \\
\text { (dBA/m) }\end{array}$ \\
\hline 50 & $7.8081 \mathrm{e}-05$ & 800 & $3.9070 \mathrm{e}-03$ \\
63 & $1.2245 \mathrm{e}-04$ & 1000 & $4.6647 \mathrm{e}-03$ \\
80 & $1.9355 \mathrm{e}-04$ & 1250 & $5.7114 \mathrm{e}-03$ \\
100 & $2.9387 \mathrm{e}-04$ & 1600 & $7.4506 \mathrm{e}-03$ \\
125 & $4.3979 \mathrm{e}-04$ & 2000 & $9.8870 \mathrm{e}-03$ \\
160 & $6.7073 \mathrm{e}-04$ & 2500 & $1.3640 \mathrm{e}-02$ \\
200 & $9.5388 \mathrm{e}-04$ & 3150 & $1.9711 \mathrm{e}-02$ \\
250 & $1.3097 \mathrm{e}-03$ & 4000 & $2.9666 \mathrm{e}-02$ \\
315 & $1.7436 \mathrm{e}-03$ & 5000 & $4.4239 \mathrm{e}-02$ \\
400 & $2.2389 \mathrm{e}-03$ & 6300 & $6.7625 \mathrm{e}-02$ \\
500 & $2.7281 \mathrm{e}-03$ & 8000 & $1.0529 \mathrm{e}-01$ \\
630 & $3.2678 \mathrm{e}-03$ & 10000 & $1.5884 \mathrm{e}-01$ \\
\hline
\end{tabular}

Source: (TNM Technical Manual)

$\rightarrow$ Frequency $1 \mathrm{~K} \rightarrow$-Frequency $2 \mathrm{~K} \rightarrow$ Frequency $3 \mathrm{~K} \rightarrow$ Frequency $4 \mathrm{~K}$

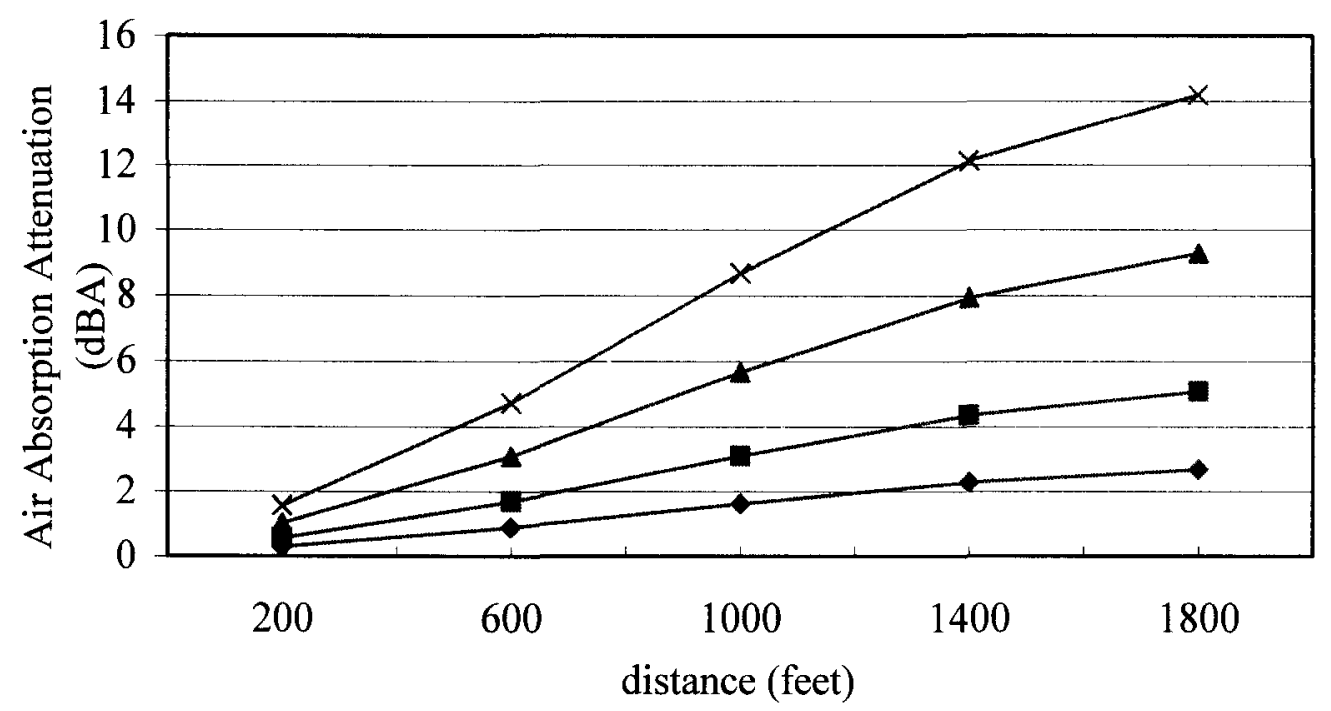

Figure 3-3. Air absorption attenuation as a function of distance 


\subsection{Insertion Loss}

In practical situations, it is important to have a reliable method of measuring the acoustical performance of roadside barriers. In the United States, the method for determining the insertion loss of outdoor noise barriers is documented in the American National Standard ANSI S12.8-1998 and Measurement of Highway-Related Noise (Lee, 1996). These standards adopt insertion loss as the basis to evaluate the acoustical effectiveness of an outdoor noise barrier. Methods are provided to determine the insertion loss of outdoor noise barriers at the selected receiver locations and under conditions of interest.

Three methods for determining the insertion loss of outdoor noise barriers are proposed in ANSI S12.8-1998: direct measured method, indirect measured method, and indirect predicted method. The preferred method is the "direct measured" method and it is the most accurate method of the three; however, this method may be used only if the barrier has not yet been installed or can be removed for the BEFORE measurements. The alternative method for direct measured method is indirect measured method. This method applies when the BEFORE condition may be simulated at a site that is equivalent to the site with the barrier. To validly determinate insertion loss, several circumstances should be met for the equivalent conditions. For example, the equivalence of the sound sources for the BEFORE and AFTER measurements. The sound sources observed in this study are mainly due to highway traffic noise that is difficult to control. In order to minimize the effect of source characteristics, a reference microphone was used to monitor the sound source for equivalence in the BEFORE and AFTER tests. The standards require that when the outdoor noise barrier is more than $15 \mathrm{~m}(50 \mathrm{ft})$ from the near edge of the 
sound-source region, the reference microphone be placed $1.5 \mathrm{~m}(5 \mathrm{ft})$ directly above the top of the barrier. Maintaining a high position of the microphone can minimize the influence by sound scattering on the noise barrier. In addition, the ground influence due to sound reflection on the varying ground impedance can be minimized. Furthermore, the equivalence of the terrain, the ground, and the atmospheric conditions at the BEFORE and AFTER sites shall be achieved. The background noise level also shall be determined and reported. When the sound level from the source of interest is at least $10 \mathrm{dBA}$ greater than the level of the corresponding background noise, no adjustment for background noise contribution need to be made. If the background noise level is $4 \mathrm{dBA}$ to $9 \mathrm{dBA}$ below the measured data, an adjustment should be added to the measured source sound level. If the background noise level is within $4 \mathrm{dBA}$ of a measured sound level, no background noise adjustment needs to be made. However, the unadjusted source sound levels shall be identified as "masked" by the background noise and lower bound for insertion loss needs to be determined.

The indirect predicted method is the last resort for determination of insertion loss due to prediction inaccuracy. This method can be applied when it is not possible to measure actual BEFORE sound levels or to have an equivalent site for the BEFORE measurements. The prediction of sound level of BEFORE condition is then calculated based on the prediction model. The standards do not prescribe specific prediction models for indirect predicted method. However, the prediction method shall consider the ground types, air conditions and include field validation in the Test Report. 
For each measurement repetition and BEFORE/AFTER pair, the insertion loss, or its lower bound, should be determined by subtracting the difference adjusted reference receiver levels (Cohn, 1998):

$$
I L=\left(L_{\text {Aref }}+L_{\text {edge }}-L_{\text {Arec }}\right)-\left(L_{\text {Bref }}-L_{\text {Brec }}\right)
$$

where,

$I L \quad$ the insertion loss at the receiver

$L_{\text {Bref }}, L_{\text {Aref }}$ the BEFORE and AFTER adjusted reference levels, respectively

$L_{\text {edge }} \quad$ the adjustments to the measured AFTER sound level at the reference microphone for effects of reflects or edge diffraction

$L_{\text {Brec }}, L_{\text {Arec }}$ the BEFORE and AFTER adjusted source levels at the receivers, respectively

For example,

$L_{\text {Aref }}=76.2 \mathrm{dBA}$

$L_{\text {edge }}=-0.5 \mathrm{dBA}$

$L_{\text {Arec }}=54.3 \mathrm{dBA}$

$L_{\text {Bref }}=75.7 \mathrm{dBA}$

$L_{\text {Brec }}=63.0 \mathrm{dBA}$

Therefore,

$\mathrm{IL}=(76.2-0.5-54.3)-(75.7-63.0)=21.4-(12.7)=8.7 \mathrm{dBA}$ 


\section{CHAPTER IV}

\section{THEORETICAL ANALYSIS}

\subsection{Introduction}

The idea of diffraction was first suggested by Young and Fresnel in the $18^{\text {th }}$ century. Wave diffraction problems were initially introduced in the area of optics, and then extended to acoustics. The exact solutions for diffraction are hard to obtain. In 1896, Sommerfeld formulated the first mathematically rigorous solution of a half plane diffraction problem. Subsequently, MacDonald (1915) extended Sommerfeld's approach to solve generalized wedge diffraction problems for cylindrical and spherical incident waves by using a spherical polar coordinate system. The solution contained integrals that were related to representation of the Hankel function (Sommerfeld, 1954). When both a source and a receiver were far from the half plane, MacDonald derived an asymptotic solution for this case in terms of Fresnel integrals. Later, Bowman and Senior (1969) recast MacDonald's solution into the cylindrical polar system to give the total field as the sum of two contour integrals. Hadden and Pierce (1974) investigated diffraction patterns around a semi-infinite wedge and gave an accurate integral solution, in which four diffracted paths contributed to the diffraction field. Their exact solution allowed for reflections of the source and receivers on the faces of the wedge by incorporating appropriate spherical wave reflection coefficients for the paths from the image sources. 
Among many approximate analytical solutions, Fresnel-Kirchhoff's approximation (Bowman, 1969) is the most common for diffraction by a half plane. It is a mathematical representation of the Huygens-Fresnel principle. By solving the Helmholtz equation with the help of Green's theorem, the sound field behind the screen can be expressed as the surface integrals of the open aperture above the screen.

In the mid-20th century, many empirical formulas were developed through indoor scale-model experiments for practical engineering applications. In the 1940s, Redfearn (1940) introduced his graph for sound attenuation behind a rigid barrier due to a point source. The parameters, the effective height of barriers normalized by the wavelength and the angle of diffraction, were used as a function for noise attenuation. A family of curves with varying diffraction angles was given by Redfearn for noise attenuation. In 1968, Maekawa (1968) presented his famous design chart that plotted the attenuation against a single parameter as Fresnel number. The Fresnel number was the numerical ratio of the path difference to the half of a sound wavelength. Not only his method can be applied for semi-infinite screen, but also can be applied for finite size screen. Ground effect can also be included for calculation. Keller applied the geometrical theory of diffraction to address barrier problems. Kurze and Anderson (1971) reviewed Keller's diffraction theory (1962) and developed empirical formulas for barrier attenuation with the help of Maekawa's and Redfearn's experimental data. Due to their simplicity, Maekawa's design chart and Kurze-Anderson's formulas have been widely used in practical engineering areas. Later, Yamamoto and Takagi (1992) improved Maekawa's original chart by developing a set of more accurate formulas, with maximum difference of less than $0.5 \mathrm{dBA}$ from the Maekawa chart. More recently, Menounon (2001) modified Maekawa's chart from a 
single curve with one parameter to a family of curves with two Fresnel numbers. Menounon also modified the Kurze-Anderson empirical formula and the Kirchhoff approximate solution for better accuracy.

Before the 1970s, interest was focused on the semi-infinite rigid plane without interference of ground. However, because much more attention was put on environmental protection, considerable outdoor noise barriers have been established since then. As a matter of fact, outdoor noise barriers were erected on the ground, and this would change the attenuation behind the barrier. Therefore, study interests changed to the overall performance of outdoor noise barriers based on practical geometrical configurations.

The ground effect in sound propagation is determined by the impedance of the ground and by the geometrical conditions. The ground behavior is strongly dependent on frequency. Delany and Bazley (1970) developed an impedance model as a function of frequency and the effective flow resistivity (EFR) to describe how the impedance varied with frequency.

The solution for the total sound pressure due to a point source above a locally reacting plane has been studied widely. The propagation of spherical sound waves over the ground was first solved approximately by Weyl (1919). By assuming that the ground surface was locally reacting and thus can be characterized by its acoustic point impedance, Ingard (1951) achieved an exact solution by using Weyl's theories. Later, other authors such as Chessell (1977), Embleton (1976), Chien and Soroka (1980) have made additional modifications. The laboratory measurements on the acoustic wave propagated along a sound-absorbing boundary that were performed by Delany and Bazley (1970) were in excellent agreement with Ingard's theories. Extensive 
measurements of the horizontal propagation of jet engine noise over the grassland were carried out by Parkin and Scholes (1964) and Scholes and Parkin (1967).

Ground type between the source and the receiver will not always be of single impedance. For example, when considering a roadway with a grass field next to it, the sound source is above the high impedance surface, the asphalt, and the receiver will be above a low impedance surface, the grass. The sound wave will interact with two different ground types. Therefore, ground with impedance discontinuities will affect the total sound attenuation. To address this practical issue, De Jong (1983) presented a semiempirical model to deal with impedance discontinuities, and it worked well with most cases. Rasmussen (1981) also developed a numerical method to deal with impedance discontinuities. This method was more complex and generated more accurate results, yet it was much more time consuming than De Jong's method. In 1995, Hothersall (1995) developed the Fresnel-zone model, which was a rather straightforward method to deal with impedance discontinuities.

To combine these two effects, barrier diffraction and ground attenuation, into a single model for the sound propagation around screens on the ground, a "heuristic" solution should be found for practical applications. Jonasson (1972) proposed a model to calculate the total sound pressure at the receiver by a superposition of four contributions, corresponding to the propagation paths between source (image source) and receiver (image receiver). This model was validated by the scale model. In the following sections, a theoretical review on the noise propagation and diffraction by semi-infinite screen will be made. 


\subsection{Free Field}

To study the sound propagation over ground surface and over noise barriers, point source propagation in free field is fundamental. Neglecting attenuation and non-linearity, free field sound propagation in air is given by wave equation (Pierce, 1989):

$$
\left(\nabla^{2}+k^{2}\right) \Phi=0
$$

where,

$\Phi \quad$ the velocity potential

$k \quad$ the wave number

$\nabla^{2} \quad$ the divergence of the gradient

A time-dependence, $e^{-i w t}$, is assumed and omitted. The sound pressure $p$ and particle-velocity $u$ are given by:

$$
\begin{gathered}
p=-\rho_{0} \frac{\partial \Phi}{\partial t} \\
u=\nabla \Phi
\end{gathered}
$$

where,

$\rho_{0} \quad$ the density of air

Solving in spherical polar co-ordinates, a point source thus produces spherical waves described as follows:

$$
p=e^{i k r} / r
$$

So the pressure amplitude falls off inversely with radial distance $r$. 


\subsection{Reflection on Level Terrain}

The simplest form for sound propagation is from a point source propagating over a level impedance surface. Assuming noise propagation from a point source above a rigid ground, which is regarded as an infinitely rigid boundary, two propagation paths can be formulated. One is the direct path from the source to the receiver; the other is the reflected path from the image source to the receiver. These two paths will interfere with each other, which result in ground effect dips. If the ground is of specific acoustic impedance, the reflected ray will be affected both in amplitude and in phase, which changes the overall frequency responses.

\subsubsection{Definition of Acoustic Impedance}

The specific acoustic (surface) impedance is defined as the complex ratio of the effective sound pressure at a point of an acoustic medium to the effective particle velocity normal to the surface at that point. Specific acoustic impedance is expressed in Equation (4-3):

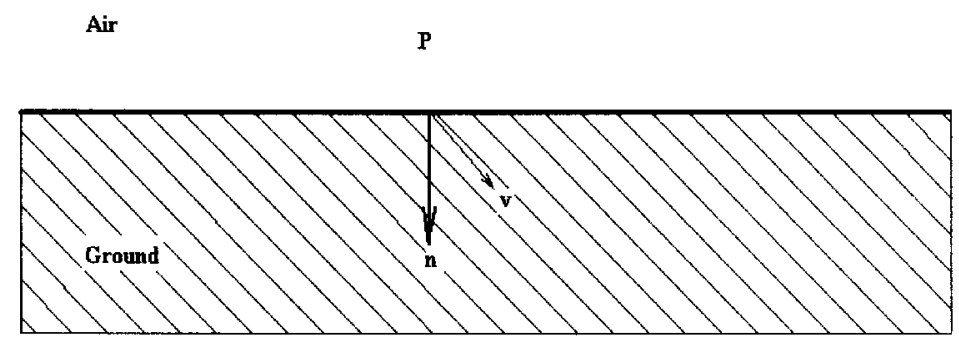

Figure 4-1. Definition of specific acoustic impedance 


$$
Z=\frac{p}{v \cdot n}
$$

Generally, surface impedance is characterized by normalizing to the impedance of

air.

$$
Z_{\text {norm }}=Z / \rho_{0} c
$$

where,

$$
\begin{aligned}
& \rho_{0} \quad \text { the density of air } \\
& c \quad \text { the speed of sound }
\end{aligned}
$$

There are two kinds of reactions by which the boundary surface responds to an incident sound pressure. One is local reaction, which means the particle velocity at a point depends only on the pressure, $p$, at the same point. The assumption that a surface is local reaction is the same as the assumption that the impedance of the surface is independent of the angle of incidence.

The other reaction is called extended reaction. Under this condition, the wave propagation within the boundary medium needs to be taken into account. In most cases local reaction at the ground surface is assumed, because the extended reaction is much more difficult to treat mathematically. Rasmussen demonstrated that the extended reaction expression yielded results very similar to the corresponding local reaction calculations with Chessell's model, and their difference was less than $0.4 \mathrm{dBA}$ in the frequency range from $100 \mathrm{~Hz}$ to $4 \mathrm{kHz}$ (Rasmussen, 1981).

\subsubsection{Impedance Models}

The ground impedance is frequency dependent and is also related to other physical parameters, such as flow resistivity or porosity. Several different ground models 
described the acoustical surface impedance of the ground. The simplest model was originated with Delany and Bazley (1971) based on their semi-empirical theories describing porous materials (1970). This model was based on the assumptions that the ground may be described as an infinitely thick layer of porous material, which can be described by flow resistance. Later, Chessell (1977) extended these ideas and used them with the concept of local reaction. According to Delany and Bazley's model, the normalized ground impedance is given as:

$$
Z_{\infty}=1+9.08\left(\frac{f}{\sigma}\right)^{-0.75}+i 11.9\left(\frac{f}{\sigma}\right)^{-0.73}
$$

where,

$f \quad$ the frequency in $\mathrm{Hz}$

$\sigma \quad$ the specific flow resistance per unit thickness in cgs units, cgs unit is based on the centimeter, the gram, and the second system

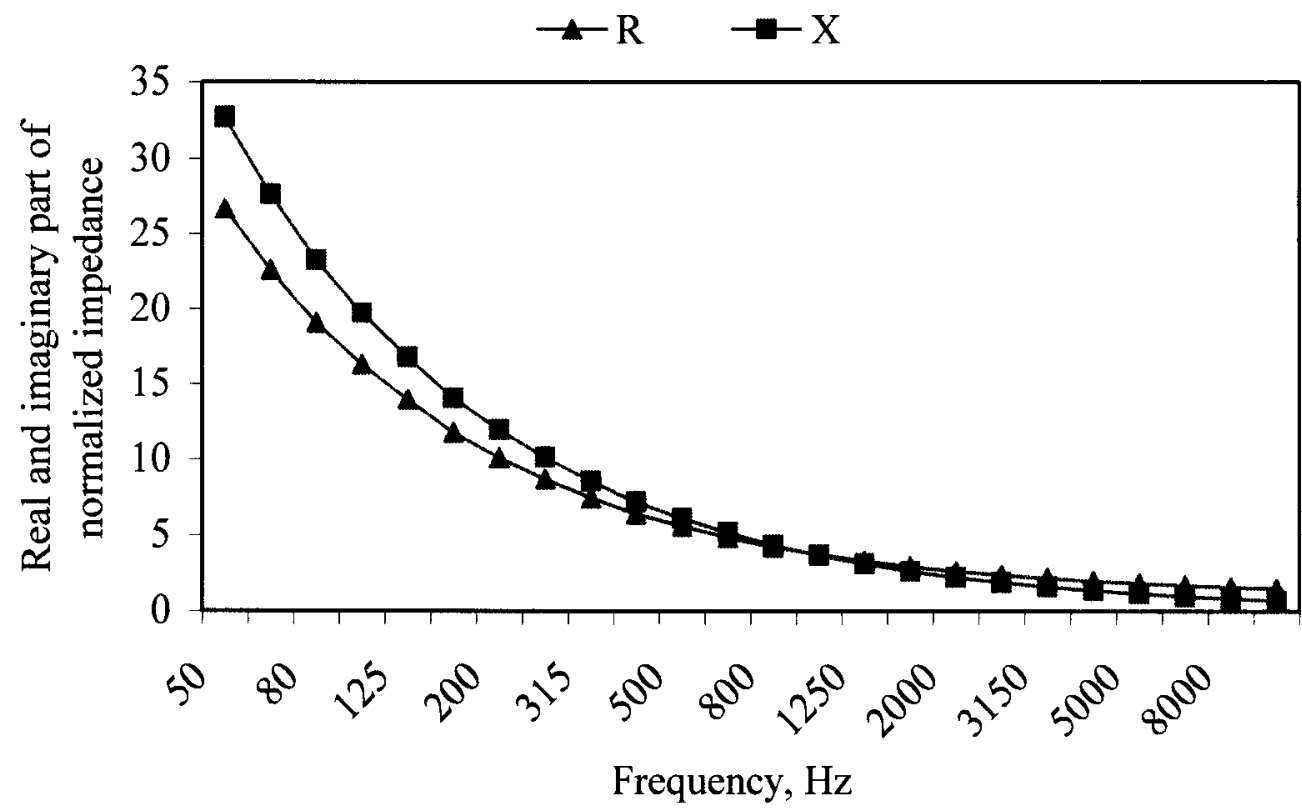

Figure 4-2. Normalized impedance value $(Z=R+i X)$ with $\sigma=200 \mathrm{cgs}$ Rayls 
Figure 4-2 is a graphical representation of normalized impedance value with $\sigma=200$ cgs Rayls. As can be seen, the acoustic impedance of the ground surface increases at low frequencies, acting as the surface becomes "harder."

This is a one-parameter model to describe ground impedance. An extension to this model used by Chessell is based on the assumptions that the porous layer is of finite depth and backed by a hard surface. In this case, the impedance model of a layer of thickness $L$ becomes:

$$
Z_{L .}=Z_{\infty} i \cot (L k), \quad k=(2 \pi f / c)\left[1+10.8\left(\frac{f}{\sigma}\right)^{-0.70}+i 10.3\left(\frac{f}{\sigma}\right)^{-0.5}\right]
$$

where,

$c \quad$ the speed of sound in air

$k \quad$ the complex wave number given from Delany and Bazley (1970)

Another two-layer impedance model developed by Thomasson (1977) is given as follows:

$$
Z_{T}=i(1+i c / \omega)^{1 / 2} /\left(a e^{i b} \tan \left[e^{i b}(1+i c / \omega)^{1 / 2} \omega / 2 \pi d\right)\right.
$$

where,

$a, b, c, d$ constants and needed to be determined by means of curve-fitting

$\omega \quad$ the radian frequency

This model utilizes $\alpha$, the effective flow resistivity (EFR), as a parameter. This model predicts high impedance for low frequencies and low impedance for high frequencies. See table 4-1 for flow resistivities of various ground surfaces. 
Table 4-1. Ground Type and Effective Flow Resistivity

\begin{tabular}{lc}
\hline Ground Type Name & Effective Flow Resistivity (cgs Rayls) \\
\hline Pavement & 20,000 \\
Water & 20,000 \\
Hard Soil (\& dirt road) & 5,000 \\
Loose Soil (\& gravel) & 500 \\
Lawn & 300 \\
Field Grass & 150 \\
Granular Snow & 40 \\
Powder Snow & 10 \\
\hline
\end{tabular}

Source: TNM Technical Manual

The three impedance models described above have one, two, and four degrees of freedom respectively. By using curve-fitting procedure (least squares method) to determine the parameters based on the experimental data, one would expect that more parameters would result in a more accurate model. That is to say, unless the model is completely erroneous, the best possible fit should be derived by the model with the greatest number of parameters (Rasmussen, 1981).

Rasmussen (1981) compared these three impedance models with field measurement data with both local reaction as well as the extended reaction. Rasmussen concluded that (i) the Chessell's ground model appeared to be a reasonable approximation to the actual properties of grass ground; (ii) the first interference dip around $500 \mathrm{~Hz}$ is not fully reproduced by Chessell's model, and the porous layer models tend to overestimate the sound pressure levels at frequencies below the first interference dip; and (iii) no significant effect of extended reaction was found since the results from the extended reaction and local reaction differ no more than $0.5 \mathrm{dBA}$. Therefore, 
Chessell's ground model and local reaction assumption are both applied to TNM and HNP.

\subsubsection{Plane Wave Reflection Coefficient}

Assuming a plane wave propagating towards an infinite plane, the reflection angle will be the same as the incident angle according to Snell's law, as shown in Figure 4-3. By assuming that the surface is of local reaction, the resulting reflection coefficient is as follows.

$$
R_{p}(\theta)=\frac{\cos (\theta)-Z_{1} / Z_{2}}{\cos (\theta)+Z_{1} / Z_{2}}
$$

where,

$\theta \quad$ the angle of incidence

$Z_{1} \quad$ the characteristic impedance of air

$Z_{2} \quad$ the characteristic impedance of the boundary medium

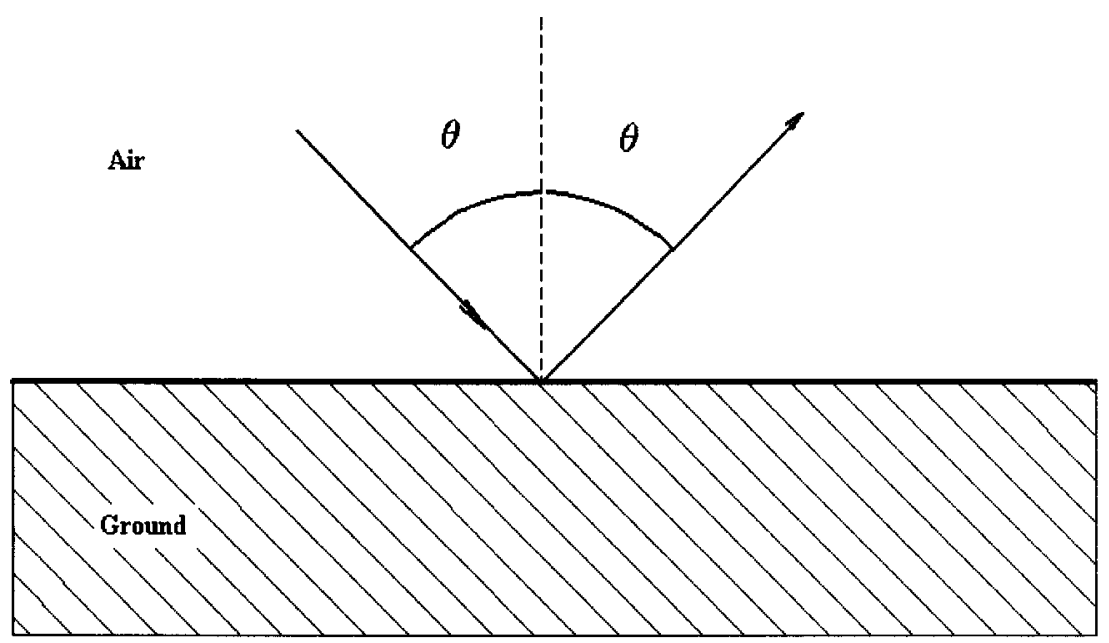

Figure 4-3. Geometry for the plane wave reflection 
In the case of extended reaction, the wave propagation within the boundary medium needs to be considered. The reflection coefficient is given by (Jonasson, 1972):

$$
R_{p}(\theta)=\frac{\cos \theta-Z_{1} / Z_{2}\left\{1-\left(c_{m} / c\right)^{2} \sin ^{2} \theta\right\}^{1 / 2}}{\cos \theta+Z_{1} / Z_{2}\left\{1-\left(c_{m} / c\right)^{2} \sin ^{2} \theta\right\}^{1 / 2}}
$$

where,

$c \quad$ the velocity of sound in air

$c_{m} \quad$ the complex wave propagation velocity

When $\left|c_{m}\right| \ll<$, Equation (4-9) can be reduced to Equation (4-8), and the boundary surface will be of local reaction.

Consider the source-receiver geometry in Figure 4-4. The sound pressure $p$ at the receiver is often expressed as:

$$
p=\frac{e^{i k R}}{R}+R \frac{e^{i k S}}{S} \approx \frac{e^{i k R}}{R}\left(1+R_{p} e^{i k \Delta r}\right)
$$

where,

$\Delta r \quad$ the path length difference

$R \quad$ the distance of the direct path from the source to the receiver

$S \quad$ the distance of the reflected path from the image source to the receiver 


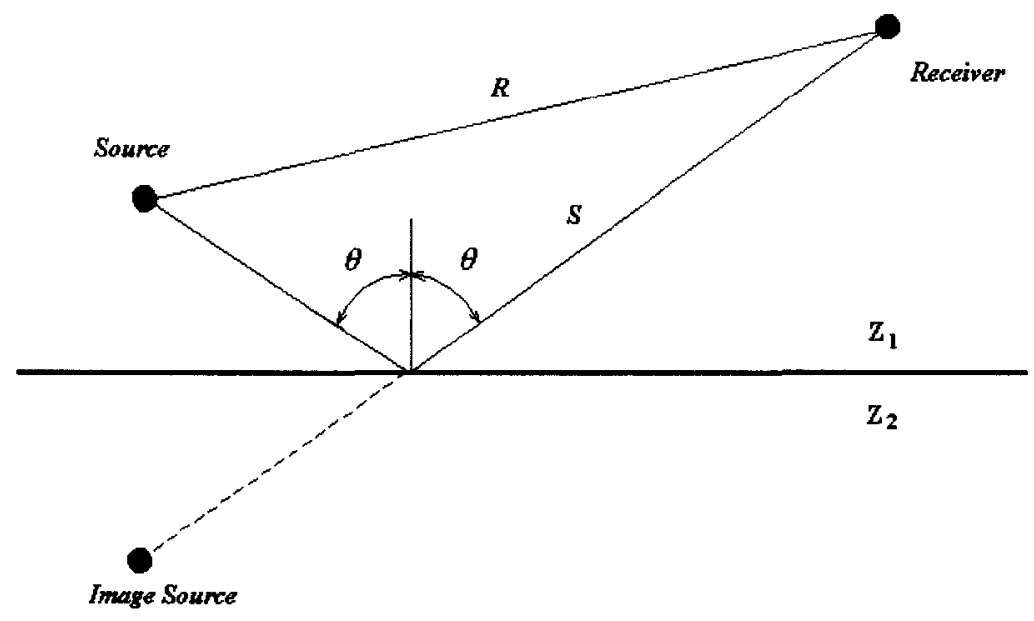

Figure 4-4. Source-receiver geometry for direct and reflected paths (De Jong, 1983)

For acoustically hard surfaces, $Z_{2}$ exceeds $Z_{1}$ by several orders of magnitude so that $R_{p}=1$ for all but large values of $\theta$. Near grazing incidence however, $\cos \theta \approx 0$, so that $R_{p}=-1$ (by Equation (4-8)). The sound pressure would vanish from Equation (4-10) since $\Delta r \approx 0$. This result is contrary to experience (Chessell, 1977).

This anomaly is resolved when the reflection of a spherical wave at the boundary instead of plane wave is considered. The sound pressure, $p$, in the case of a spherical incident wave can be expressed as:

$$
\begin{gathered}
p=\frac{e^{i k R}}{R}\left[1+Q e^{i k \Delta r}\right] \\
Q=R_{p}+F(w)\left(1-R_{p}\right)
\end{gathered}
$$

where,

$Q \quad$ the spherical reflection coefficient, or image source strength

$F(w)$ the boundary loss factor

$w \quad$ the numerical distance 
The numerical distance $w$ can be reduced to Equation (4-13) when considering the locally reacting case. The term $\cos \theta \cdot Z_{1} / Z_{2}$ in the denominator of Equation (4-13) can as a rule be neglected (Jonasson, 1972).

$$
w=\frac{1}{2} i k_{1} r_{2} \frac{\left(\cos \theta+Z_{1} / Z_{2}\right)^{2}}{\left(1+\cos \theta \cdot Z_{1} / Z_{2}\right)}
$$

According to Chessell, Equation (4-14) is used to express $F(w)$ for reasonably small values of $w$.

$$
\begin{aligned}
F(w) & =1+i \exp (-w)(\pi w)^{1 / 2}-2 \exp (-w) \sum_{n=1}^{\infty} \frac{w^{n}}{(n-1) !(2 n-1)} \\
& =1+i \exp (-w)(\pi w)^{1 / 2}-2 w \exp (-w)\left[1+\frac{w}{1 ! 3}+\frac{w^{2}}{2 ! 5}+\frac{w^{3}}{3 ! 7} \cdots\right]
\end{aligned}
$$

For larger values of $w(|w|>10)$, the asymptotic series is shown in Equation (4$15)$.

$$
F(w)=-\sum_{n=1}^{\infty} \frac{(2 n) !}{2^{n} n !(2 w)^{n}}=-\left[\frac{1}{2 w}+\frac{1 \cdot 3}{(2 w)^{2}}+\frac{1 \cdot 3 \cdot 5}{(2 w)^{3}}+\cdots\right]
$$

According to Equation (4-14) and Equation (4-15), $F(w)$ is a simplified asymptotic series. This may lead to inaccuracy when calculating reflection coefficients for certain source-receiver configurations. Figure 4-5 shows an example to calculate $F(w)$ by TNM's method. The distance between source and receiver is $100 \mathrm{~m}$; source height is $1 \mathrm{~m}$; receiver height is $1.52 \mathrm{~m}$; ground EFR is 200 cgs Rayls. 


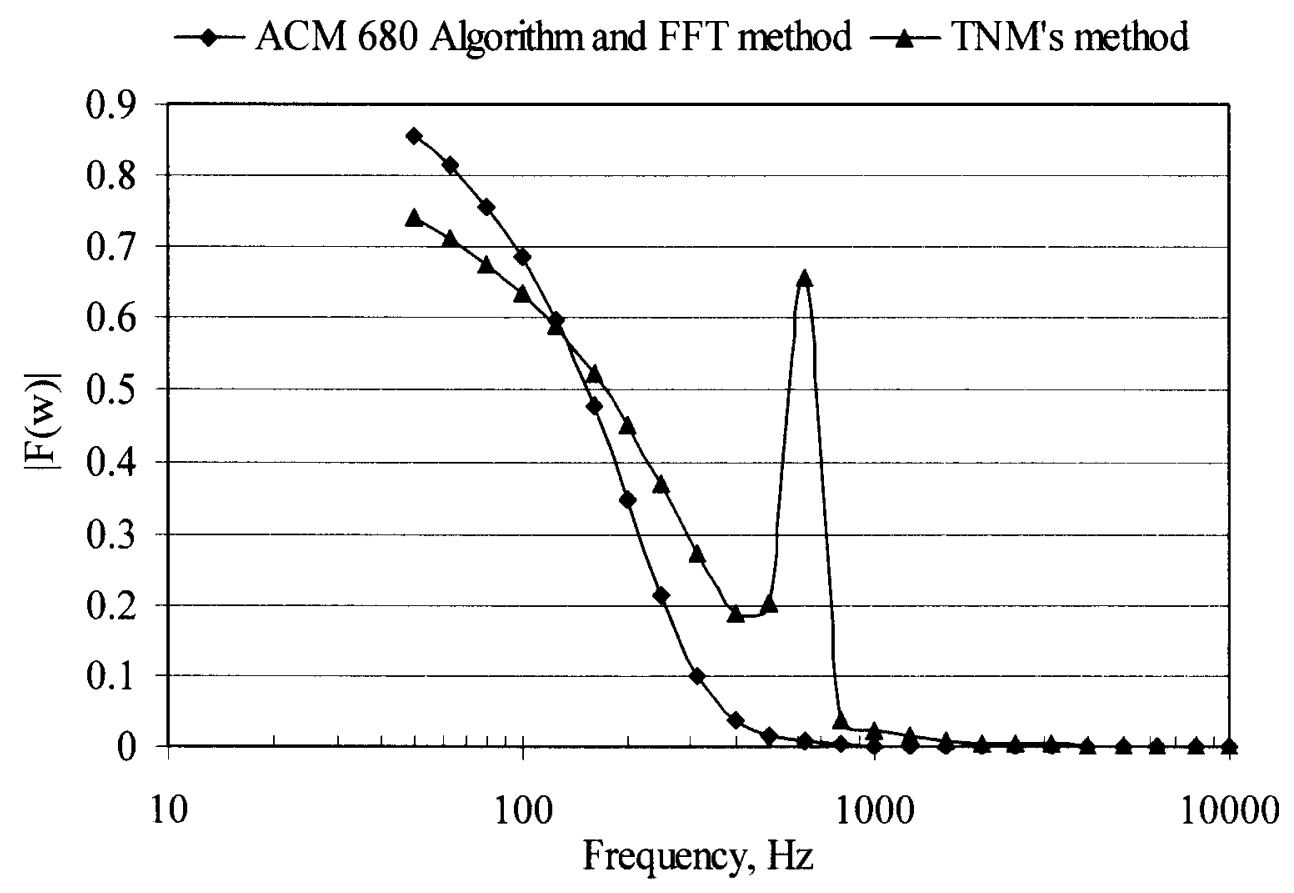

Figure 4-5. Comparison TNM's method, ACM 680 Algorithm and FFT method to calculate $F(w)$

As can be seen in Figure 4-5, y-axis denotes the absolute value of $F(w)$, which is a strictly decreasing function with increasing frequencies. However, the curve of $F(w)$ generated by TNM's method shows a sudden increase at points where the value of $w$ approaches 10.

According to Chien and Soroka (1980), the original expression for $F(w)$ can be expressed as follows:

$$
F(z)=1+i \sqrt{\pi} z e^{-z^{2}} \operatorname{erfc}(-i z)
$$

where,

$\operatorname{erfc}(z)$ the complementary error function extended for complex arguments $w(z)=e^{-z^{2}} \operatorname{erf} c(-i z)$ the complex error function

Many researchers (Chien and Soroka, 1980), (Weideman, 1994), (Poppe and 
Wijers, 1990), (M.Abramowitz and I.A.Stegun, 1970) described how to calculate the complex error function. The method developed by Poppe (1990) for mathematical computing was adopted. The algorithm 680 associated with the method was used in HNP 1.0. This method is an improved version of Gautschi's algorithm. By modifying the tuning of the algorithm and testing the relative rather than the absolute error, the accuracy of this algorithm can be improved to 14 significant digits throughout almost the whole of the complex plane. In addition, the efficiency of this algorithm is further enhanced by using a different approximation in the neighborhood of the origin.

Another method developed by Weideman is also used in the study for comparison. This method computes the complex error function using rational expansions. The polynomial coefficients can be computed by a single Fast Fourier Transform (FFT). The accuracy of this method is about 16 digits by using Matlab software.

As can be seen in Figure 4-5, the two methods are compared and agreed with each other and the calculated $F(w)$ by these two methods decrease monotonously. In addition, they behave well numerically for most normal source and receiver positions. Last but not the least, these two methods comply with the results of the Handbook of Mathematical Functions (M.Abramowitz and I.A.Stegun, 1970). On the other hand, TNM's method deviates a large error compared with ACM algorithm 680 and FFT method. Therefore, ACM algorithm 680 is applied to HNP 1.0 instead of the method of TNM. The calculated $F(w)$ for this case by TNM's method, ACM 680 algorithm and FFT method are shown in Table 4-2, Table 4-3 and Table 4-4 in Appendix I.

Figure 4-6 shows the difference between spherical and plane wave reflection coefficients. As can be seen, the noise attenuation by grassland is almost the same for the 
plane wave and the spherical wave in high frequencies, but large differences exist in low frequencies. The minimum interference occurs at the same frequency for both spherical and plane waves.

$$
\multimap \text { spherical }(\mathrm{Q}=\mathrm{Q}) \rightarrow \text { plane }(\mathrm{Q}=\mathrm{R}) \quad \begin{gathered}
\text { Source } h t .=1 \mathrm{~m} \\
\text { Receiver } h t .=1 \mathrm{~m}
\end{gathered}
$$

$\mathrm{EFR}=325 \mathrm{cgs}$ rayls, corresponding to a grass surface, distance $=100 \mathrm{~m}$

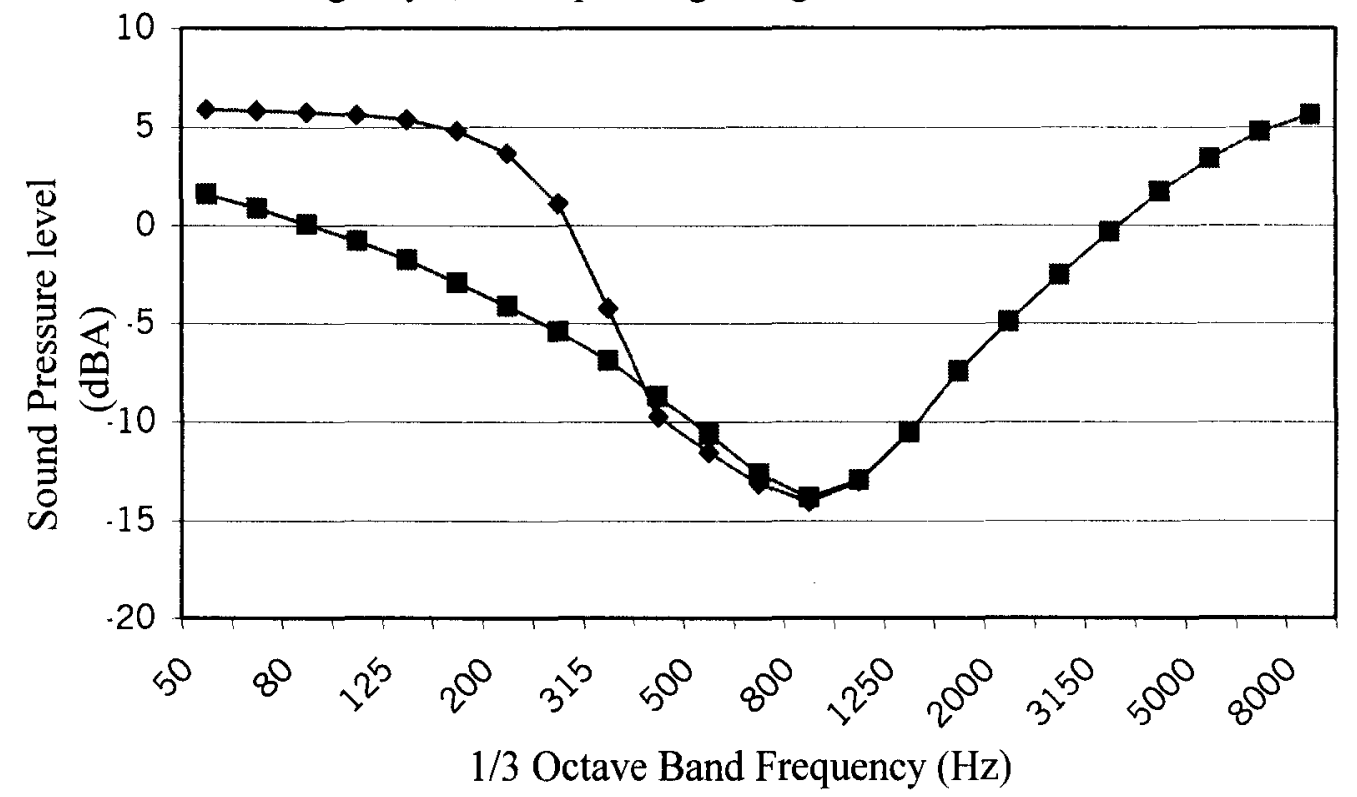

Figure 4-6. Difference between spherical and plane wave reflection coefficients

Figure 4-7 shows a graphic representation of a set of predicted spectra for various values of flow resisitivity of ground surface. Reference sound pressure level is in the free field. Source height is $0.5 \mathrm{~m}$, receiver height is $1.5 \mathrm{~m}$, and horizontal distance is $20 \mathrm{~m}$.

As can be seen in Figure 4-7, y-axis denotes sound level relative to free field, also known as the excess attenuation. The excess attenuation is a function of frequency. An increase in the flow resistivity causes a reduction in the excess attenuation and a shift in the peak attenuation value to higher frequencies when flow resistivity ranges from $10 \mathrm{cgs}$ 
Rayls to 1,000 cgs Rayls. However, when flow resistivity is 10,000 cgs Rayls, the excess attenuation becomes greater.

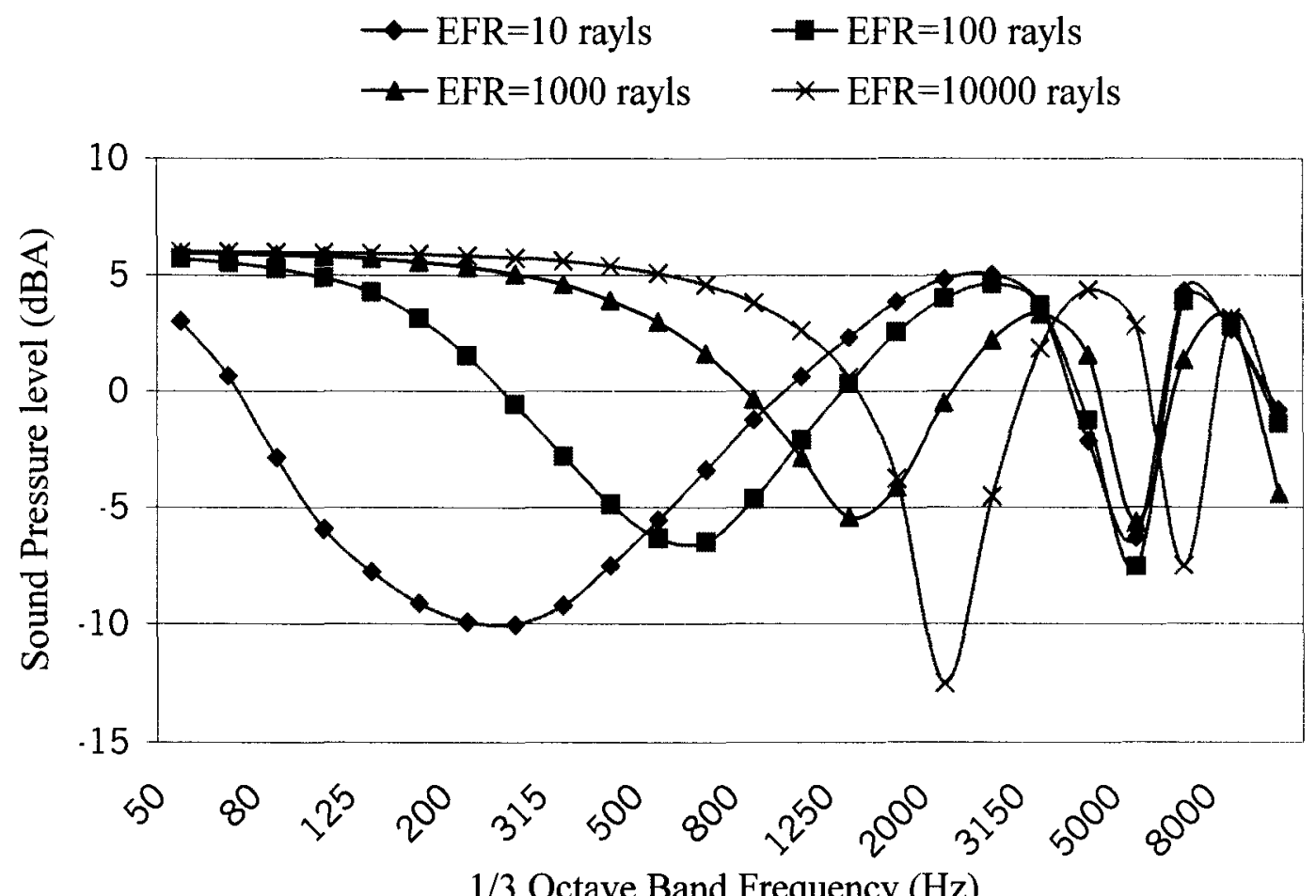

Figure 4-7. Set of predicted spectra for various values of flow resisitivity of ground surface

Another conclusion related to Figure 4-7 is that the attenuation at an interference minimum is a relatively rapidly varying function of effective flow resistivity; however, it depends very little on the source and receiver heights, or their separation distance. The frequency of at which the interference minimum occurs may depend to a greater extent on such geometrical considerations (Embleton, 1983).

\subsection{Mathematical Formulation for Diffraction}

In order to seek a solution for the sound field due to a point source in the vicinity of a semi-infinite plane, first a proper coordinate is selected and the geometrical configuration of the problem needs to be considered. A cylindrical polar coordinate 
system is selected for convenience in describing the relation of source, receiver and the semi-infinite plane. The origin is located at the edge and $(r, \theta, z)$ is the cylindrical polar coordinates. The z-axis coincides with the edge of the half plane, the initial line of the polar coordinates $(\theta=0)$ lies on the right-hand surface of the half plane, and the end line of the polar coordinates $(\theta=2 \pi)$ lies on the left-hand surface of the half plane. All radial distances are measured from the edge of the half plane and all angular positions are measured in a counter-clockwise direction.

Using the principle of geometrical acoustics, the total sound pressure consists of the pressure due to the diffracted wave, $p_{d}$, and a geometrical solution that combines the pressure of direct and reflected waves, $p_{i}$, and $p_{r}$, as shown in Figure 4-8. The dashed lines at $\theta=3 \pi-\theta_{s}$ and $\theta=\theta_{s}-\pi$ divide the sound field into three separate regions I, II and III. The reflected ray's path cannot be constructed in regions II and III. The direct ray's path cannot be constructed in region III because of the presence of the thin plane that prevents direct line-of-sight between source and receiver. The diffracted ray's path can be constructed in all three regions. The dividing line at $\theta=\theta_{s}-\pi$ separates region II from region III and is called the shadow boundary. When the source is located to the lefthand surface of the thin plane, the occurrence of each wave type can be illustrated as follows:

1. A direct wave is included if $\pi-\left|\theta_{r}-\theta_{s}\right| \geq 0$

2. A reflected wave from the face of $\theta=2 \pi$ if $\left(\theta_{r}+\theta_{s}\right)-3 \pi \geq 0$

3. A diffracted wave from edge of the plane if $0 \leq \theta_{s}, \theta_{r} \leq 2 \pi$ 


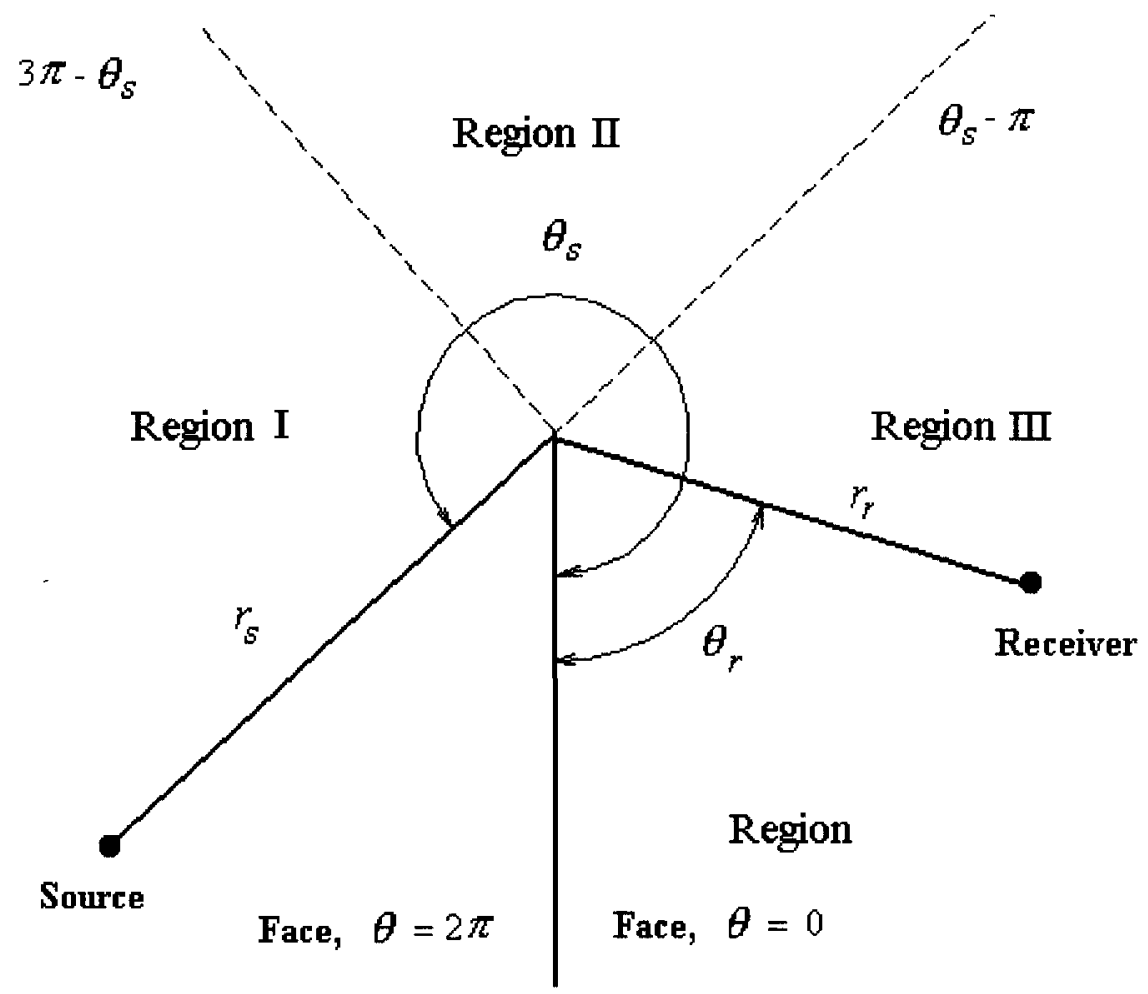

Figure 4-8. Schematic diagram for the different section of sound field

Therefore, from the perspective of geometrical acoustics, the sound field in the vicinity of the half plane can be represented in terms of three parts. The first term accounts for the contribution of the direct wave $p_{i}$, the second term accounts for the contribution of the reflected wave $p_{r}$ and the last term accounts for the contribution of the diffracted wave at the edge $p_{d}$. The total sound field $p_{T}$ in each region is given as follows:

Region I: $p_{T}=p_{i}+p_{r}+p_{d}$

Region II: $p_{T}=p_{i}+p_{d}$

Region III: $p_{T}=p_{d}$

One should note that if only the diffracted wave is considered, it will not be continuous at the boundaries between the regions. 


\subsubsection{The Fresnel Integral}

In the theory of diffraction of a semi-infinite plane, the Fresnel integral and other related functions are very important for numerical computations. The definition and the plot of the Fresnel integral are shown in Equation (4-17), Figure 4-9, and Figure 4-10:

$$
F(x)=\int_{x}^{\infty} e^{i t^{2}} d t
$$

When calculating the Fresnel integral with a negative number, Equation (4-18) can be used:

$$
F(-x)=\sqrt{\frac{\pi}{2}}(1+i)-F(x)
$$

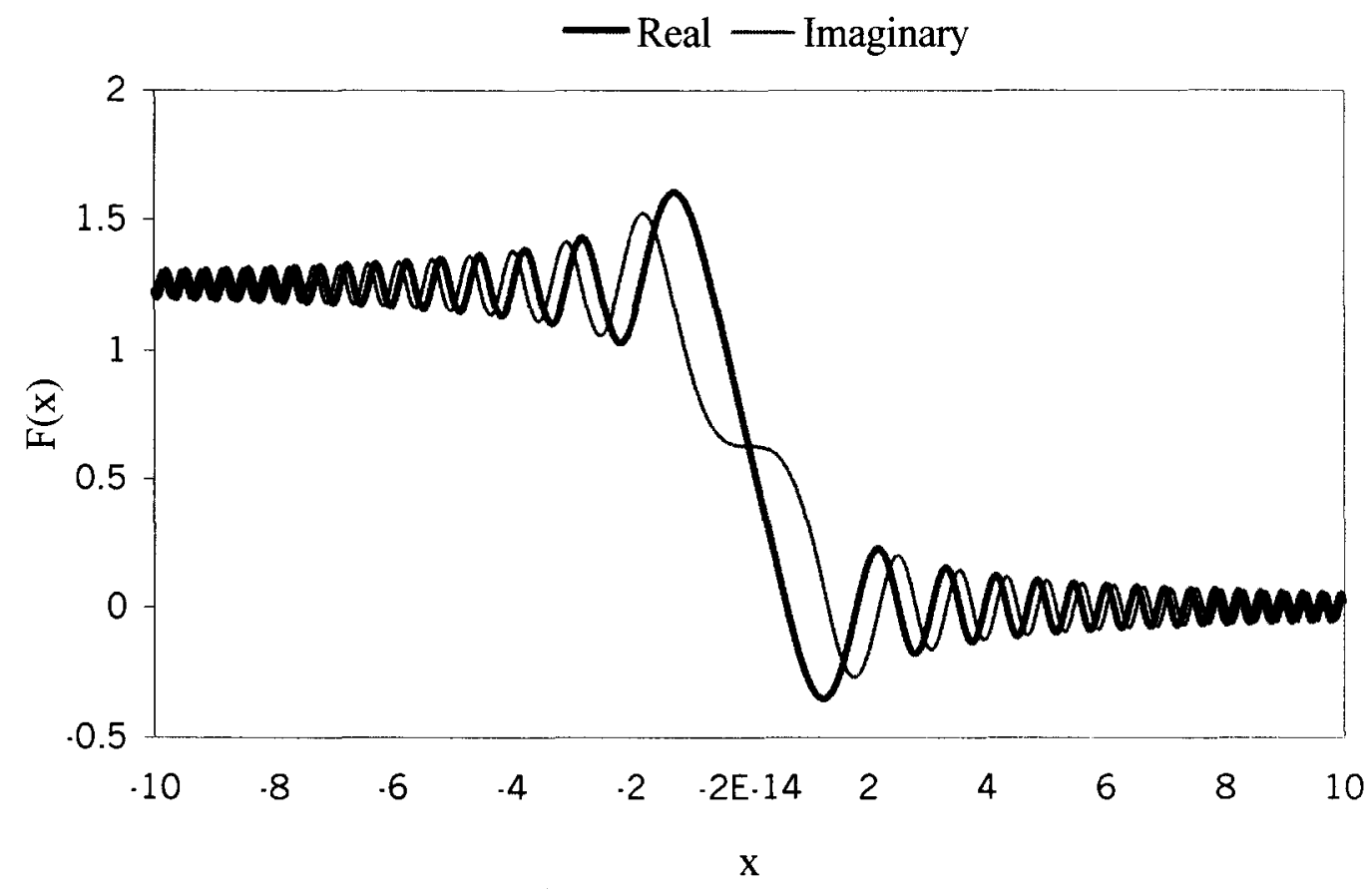

Figure 4-9. Real and imaginary parts of the Fresnel integral with $x$ between -10 and 10 


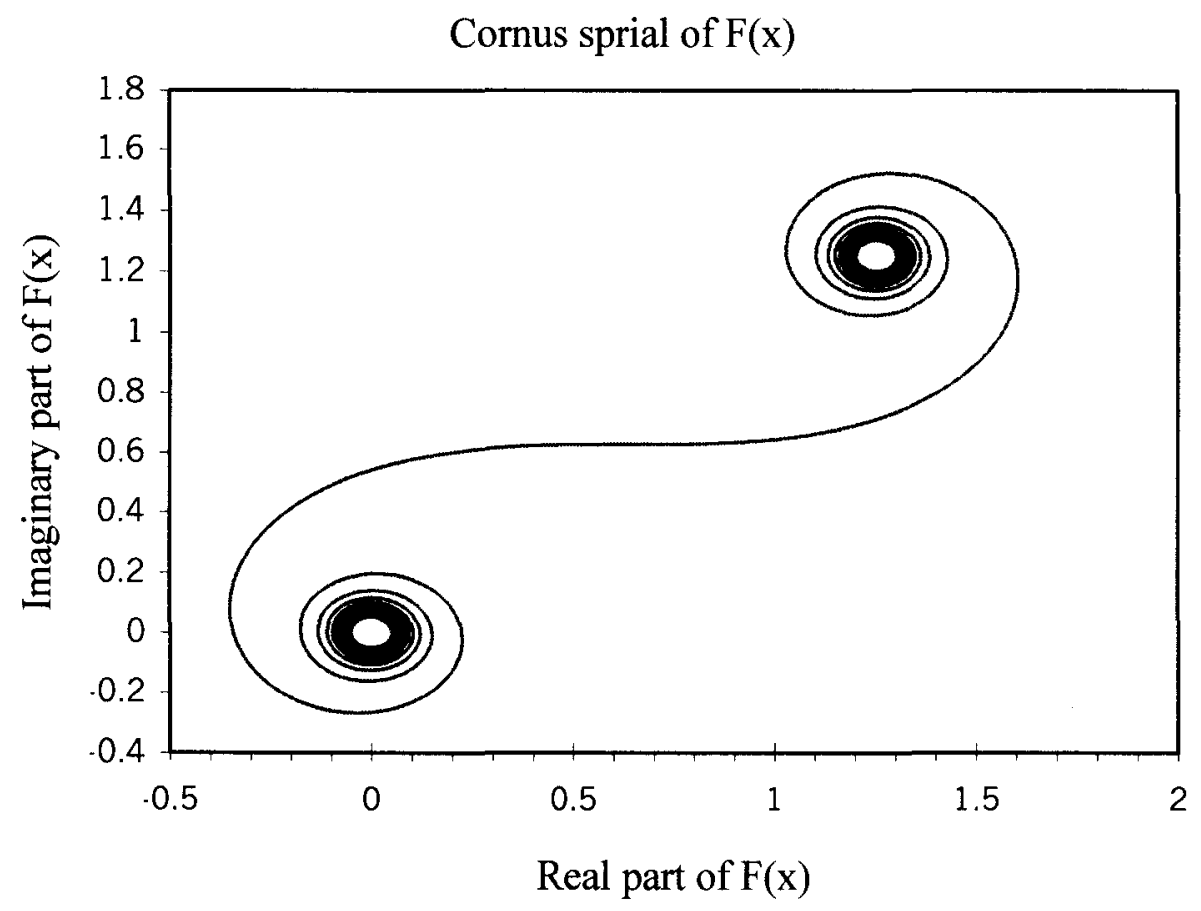

Figure 4-10. Alternative plot for the Fresnel integral $F(x)$

\subsubsection{The Fresnel-Kirchhoff Solution}

Assuming the sound wave, starts from a point source, propagates through an above the semi-infinite screen, and then reaches to the receiver, shown in Figure 4-11. According to Fresnel-Kirchhoff's diffraction theory, the sound attenuation by the screen is given as (Maekawa, 1965):

$$
U=-i A \int_{u_{1}}^{\mu_{2}} e^{i \frac{\pi}{2} u^{2}} d u \int_{l_{1}}^{v_{2}} e^{i \frac{\pi}{2} v^{2}} d v
$$

In the free field, substituting $u_{1}=-\infty, v_{1}=-\infty, u_{2}=+\infty, v_{2}=+\infty$ in Equation (419) yields Equation (4-20):

$$
U_{f f}=-i A(1+i)^{2}=2 A
$$

The ratio of sound pressure with the screen relative to free field pressure can be 
expressed by Equation (4-21):

$$
\begin{gathered}
\frac{U}{U_{f f}}=\frac{-i}{2} \int_{u_{1}}^{u_{2}} e^{i \frac{\pi}{2} u^{2}} d u \int_{d_{1}}^{v_{2}} e^{i \frac{\pi}{2} v^{2}} d v \\
=\frac{-i}{2}\left\{C\left(u_{2}\right)-C\left(u_{1}\right)+i\left(S\left(u_{2}\right)-S\left(u_{1}\right)\right)\right\} \times\left\{C\left(v_{2}\right)-C\left(v_{1}\right)+i\left(S\left(v_{2}\right)-S\left(v_{1}\right)\right)\right\}
\end{gathered}
$$

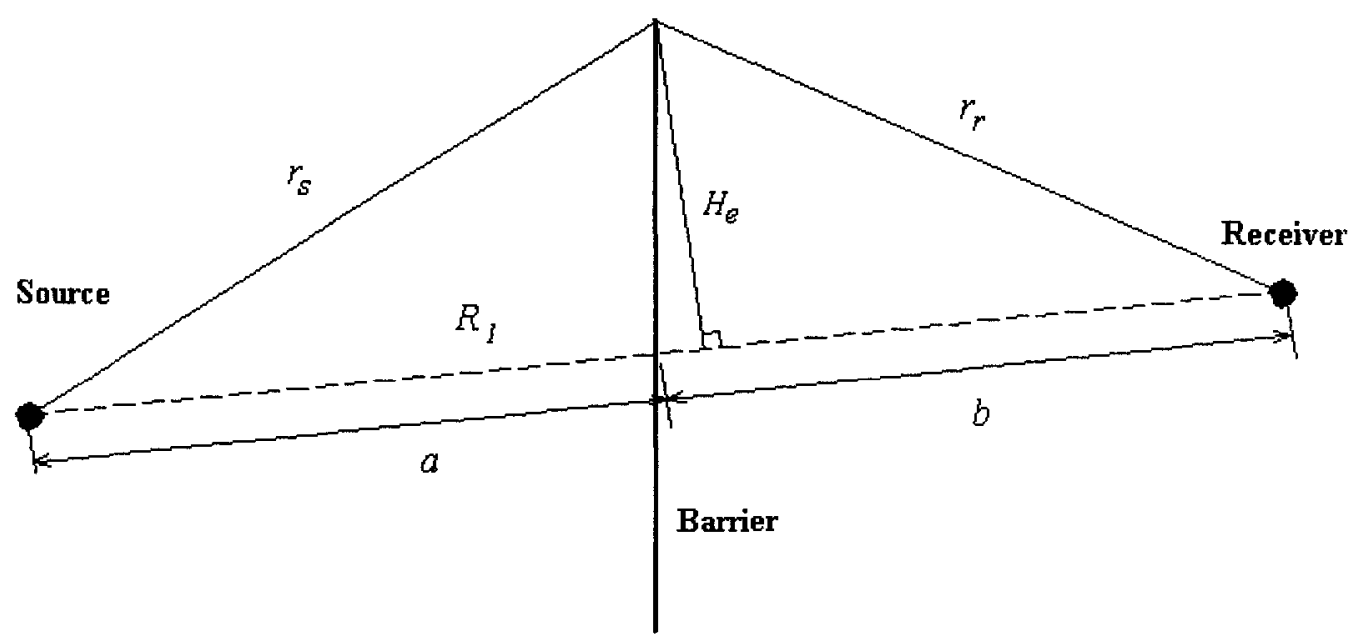

Figure 4-11. Diffraction of sound by semi-infinite plane for Fresnel-Kirchhoff's Solution

By substituting $u_{1}=-\infty, u_{2}=+\infty, v_{2}=+\infty$ in Equation (4-21), the Kirchhoff diffraction theory can be applied to the semi-infinite screen as shown in Equation (4-22):

$$
\frac{U}{U_{f f}}=\frac{-i}{2}\{1+i\}\left\{\left(\frac{1}{2}-C\left(v_{1}\right)\right)+i\left(\frac{1}{2}-S\left(v_{1}\right)\right)\right\}
$$

The insertion loss by this semi-infinite screen is given by Equation (4-23):

$$
I L=-10 \log \left|\frac{U}{U_{f f}}\right|^{2}=-20 \log \left|\frac{U}{U_{f f}}\right|
$$

where,

$C$ and $S \quad$ the Fresnel's integrals for variable $v_{1}$

$H_{e} \quad$ the effective height of the screen 
$a$ and $b \quad$ the distances from the screen to the source and the receiver, respectively

$$
\begin{gathered}
C\left(u_{1}\right)=\int_{0}^{u_{1}} \cos \left(\frac{\pi}{2} u^{2}\right) d u \\
S\left(u_{1}\right)=\int_{0}^{u_{1}} \sin \left(\frac{\pi}{2} u^{2}\right) d u \\
v_{1}=H_{e} \sqrt{\frac{2}{\lambda}\left(\frac{1}{a}+\frac{1}{b}\right)}
\end{gathered}
$$

When $v_{1}>0$, the receiver is in the shadow zone, where the line of sight between the source and the receiver is blocked by the screen. Otherwise, the receiver is in the illuminated zone.

\subsubsection{The Macdonald Solution}

De Jong (1983) presented a way to calculate the semi-infinite diffraction for an acoustically hard wedge based on an exact solution by Macdonald. The diffracted field in the shadow zone is given by:

$$
\begin{gathered}
P_{d}=D_{i} p_{i}+D_{r} p_{r} \\
p_{i}=\frac{e^{i k R}}{k R}, p_{r}=\frac{e^{i k S}}{k S} \\
D_{i}=\frac{R}{L} \frac{e^{-i \pi / 4}}{\sqrt{\pi}} e^{i k(l-R)} e^{-i \chi_{-}^{2}} F\left(\chi_{-}\right) \\
D_{r}=\frac{S}{L} \frac{e^{-i \pi / 4}}{\sqrt{\pi}} e^{i k(L-S)} e^{-i \chi_{+}^{2}} F\left(\chi_{+}\right) \\
\chi_{ \pm}=\left(\frac{k r r_{0}}{2 L}\right)^{1 / 2} \frac{\cos (\pi / v)-\cos \left[\left(\phi \pm \phi_{0}\right) / v\right]}{(1 / v) \sin (\pi / v)}
\end{gathered}
$$




$$
\begin{gathered}
L^{2}=\left(r+r_{0}\right)^{2}+\left(Z-Z_{0}\right)^{2} \\
v=(2 \pi-T) / \pi \\
F(\chi)=\int_{\chi}^{\infty} e^{i t^{2}} d t
\end{gathered}
$$

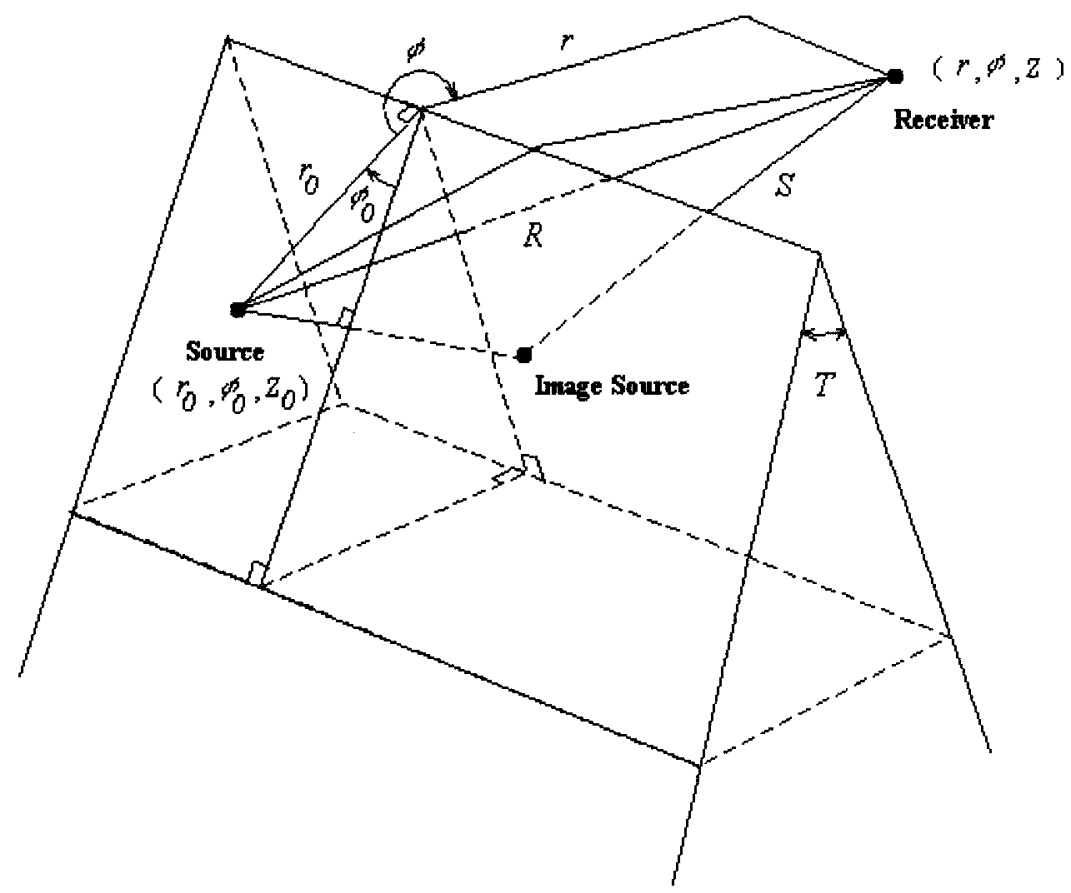

Figure 4-12. Geometry of diffraction at a hard wedge (De Jong, 1983)

where,

$D_{i} \quad$ diffraction coefficient for real source

$D_{r} \quad$ diffraction coefficient for image source

$T \quad$ top angle of the wedge

$L \quad$ distance of path from the source to the receiver over the barrier

$X_{ \pm} \quad$ argument for the relative position of the source, receiver and the edge of barrier

$Z, Z_{0} \quad \mathrm{z}$ coordinate along the barrier 
As can be seen in Figure 4-12, for an acoustically hard wedge, there exists a image source of the real source in the hard wedge. In Equation (4-27), $p_{d}$ includes two terms. The first term can be interpreted physically as the diffraction field due to the incident wave $p_{i}$, and the second term can be interpreted as the diffraction field due to the reflected wave $p_{r}$.

Theoretically these equations hold only for $k r>>1$, and this condition is always fulfilled in practical situations in which $k r>10$.

\subsubsection{Diffraction at a Semi-Infinite Screen}

The semi-infinite screen is a special case of the acoustically hard wedge described in the previous section, as shown in Figure 4-13. In the semi-infinite screen case, $v=2$ (top angle $=0$ ), the diffraction coefficients can be simplified to:

$$
\begin{gathered}
D_{i}=\frac{R}{L} \frac{e^{-i \pi / 4}}{\sqrt{\pi}} F\left(\chi_{-}\right) \\
D_{r}=\frac{S}{L} \frac{e^{-i \pi / 4}}{\sqrt{\pi}} F\left(\chi_{+}\right)
\end{gathered}
$$

One can write the diffracted field $p_{d}$ in the shadow zone as:

$$
\begin{aligned}
& p_{d}=\frac{e^{-i \pi / 4}}{\sqrt{\pi}}\left[\frac{e^{i k R}}{k L} F\left(\chi_{-}\right)+\frac{e^{i k S}}{k L} F\left(\chi_{+}\right)\right] \\
& \chi_{-}=-\frac{1}{2}\left(\frac{k r_{0} r}{2 L}\right)^{1 / 2} \cos \left(\frac{\phi-\phi_{0}}{2}\right)=\sqrt{k(L-R)} \\
& \chi_{+}=-\frac{1}{2}\left(\frac{k r_{0} r}{2 L}\right)^{1 / 2} \cos \left(\frac{\phi+\phi_{0}}{2}\right)=\sqrt{k(L-S)}
\end{aligned}
$$




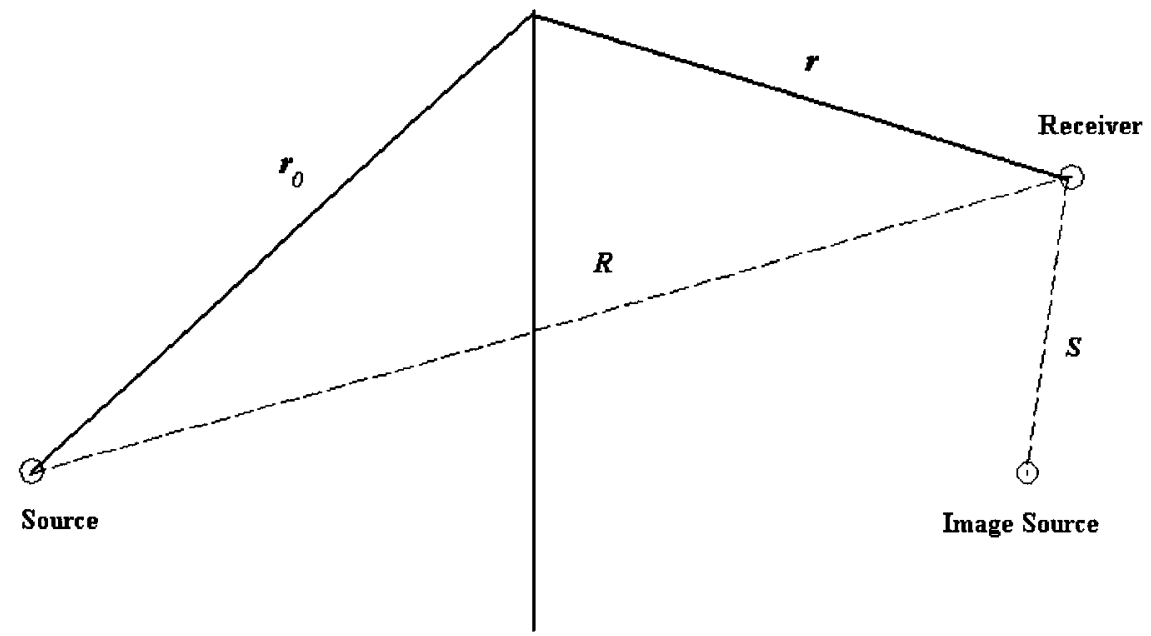

Figure 4-13. Geometry of diffraction at a semi-infinite plane

where,

$r_{0} \quad$ distance from the source to the top of the barrier

$r \quad$ distance from the top of the barrier to the receiver

$R \quad$ direct distance from the source to the receiver

$S \quad$ direct distance from the image source in the barrier to the receiver

\subsubsection{The Pierce Solution}

Pierce and Hadden (1981) derived their asymptotic solution for the diffraction of sound by a semi-infinite wedge. If the wedge angle $\beta$ is $2 \pi$, this corresponds to the semi-infinite plane case. The diffracted field can be represented by:

$$
p_{d}=\left(\frac{e^{i k L}}{K L}\right)\left(\frac{e^{i \pi / 4}}{\sqrt{2}}\right)\left[A_{D}\left(X_{+}\right)+A_{D}\left(X_{-}\right)\right]
$$

where,

$$
X_{+}=X\left(\theta+\theta_{0}\right), X_{-}=X\left(\theta-\theta_{0}\right)
$$




$$
\begin{gathered}
A_{D}(X)=\frac{\sqrt{2}}{2 \pi} \int_{-\infty}^{\infty} \frac{e^{-u^{2}}}{(\pi / 2)^{1 / 2} x-e^{-i \pi / 4} u} d u=\operatorname{sign}(x)[f|x|-i g|x|] \\
X(\theta)=\sqrt{\frac{2 r_{0} r}{\lambda L}} \frac{\cos (v \pi)-\cos (v \theta)}{v \sin (v \pi)}
\end{gathered}
$$

Based on the original version of Pierce and Hadden's solution of diffraction, the diffracted sound $p_{d}$ is made up of four possible diffraction paths:

1. from the source to the receiver

2. from the image source(due to barrier surface) to the receiver

3. from the source to the image receiver (due to barrier surface)

4. from the image source (due to barrier surface) to the image receiver (due to barrier surface)

The contribution of the first and the fourth diffraction paths are considered in terms of $A_{D}\left(X_{-}\right)$while the contribution of the second and the third diffraction paths are considered in terms of $A_{D}\left(X_{+}\right)$. The functions $f(X)$ and $g(X)$ in Equation (4-45), (446) are called auxiliary Fresnel functions and can be defined in terms of Fresnel integrals $C(X)$ and $S(X)$. Their relationship can be represented as follows:

$$
\begin{gathered}
C(X)=\int_{0}^{X} \cos \left(\frac{\pi t^{2}}{2}\right) d t \\
S(X)=\int_{0}^{X} \sin \left(\frac{\pi t^{2}}{2}\right) d t \\
f(X)=\left(\frac{1}{2}-S(X)\right) \cos \left(\frac{\pi X^{2}}{2}\right)-\left(\frac{1}{2}-C(X)\right) \sin \left(\frac{\pi X^{2}}{2}\right) \\
g(X)=\left(\frac{1}{2}-C(X)\right) \cos \left(\frac{\pi X^{2}}{2}\right)+\left(\frac{1}{2}-S(X)\right) \sin \left(\frac{\pi X^{2}}{2}\right)
\end{gathered}
$$


The use of $f(X)$ and $g(X)$ simplifies the writing of the equations and is more convenient for numerical computations. In addition, these functions are monotonic and their asymptotic values are computed readily. Therefore, $f(X)$ and $g(X)$ are used in much of the literature. Figure 4-14 shows the graphic representation of $f(X)$ and $g(X)$.

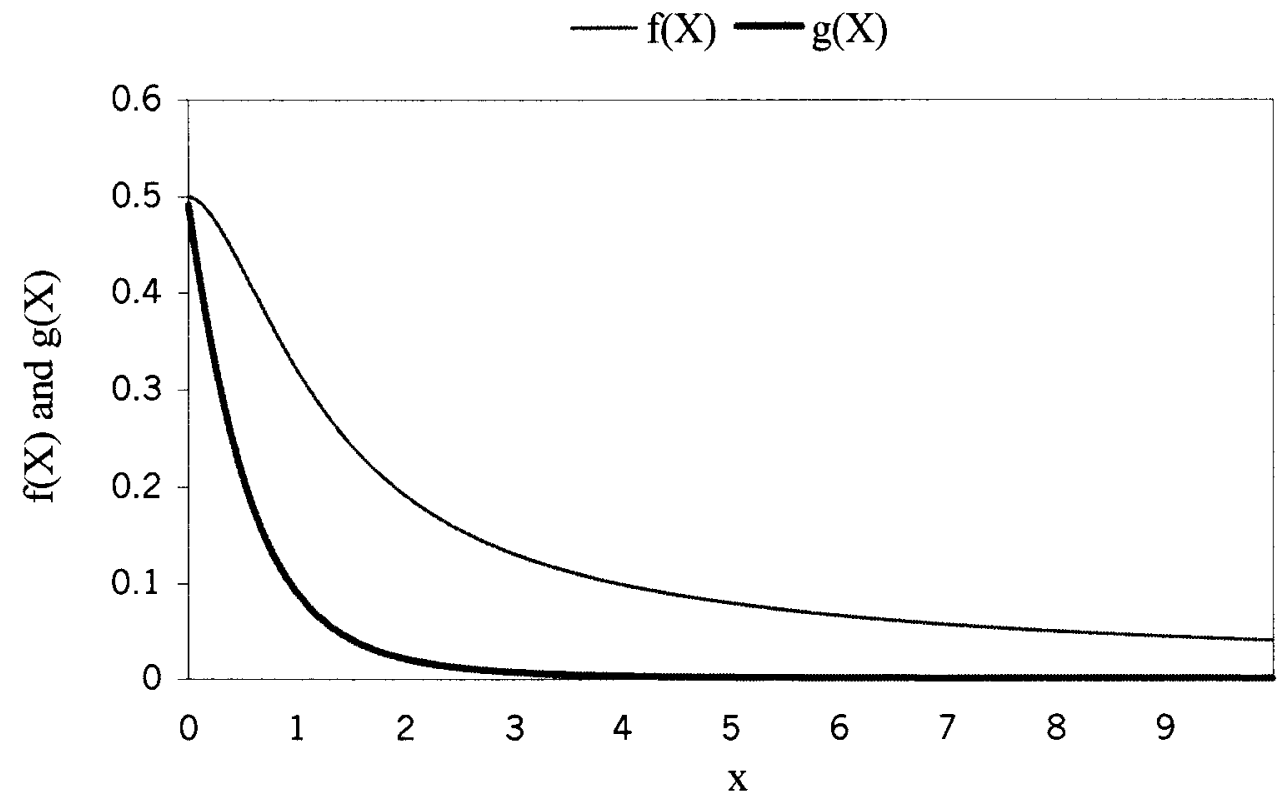

Figure 4-14. The auxiliary Fresnel function $f(X)$ and $g(X)$

\subsection{Diffraction at an Impedance Discontinuity}

In the previous section, a homogeneous ground surface is assumed for the ground reflection. However, this assumption is not always the case in practical situations. For example, highway noise, generated above a hard road surface (e.g. asphalt or concrete), propagates over a soft ground surface (e.g. grassland or lawn) and then reaches the receivers. To account for the effect of these different ground surfaces for sound propagation, it is necessary to take the diffraction occurring at the impedance discontinuity (the transition point from hard surface to soft surface) into account. 


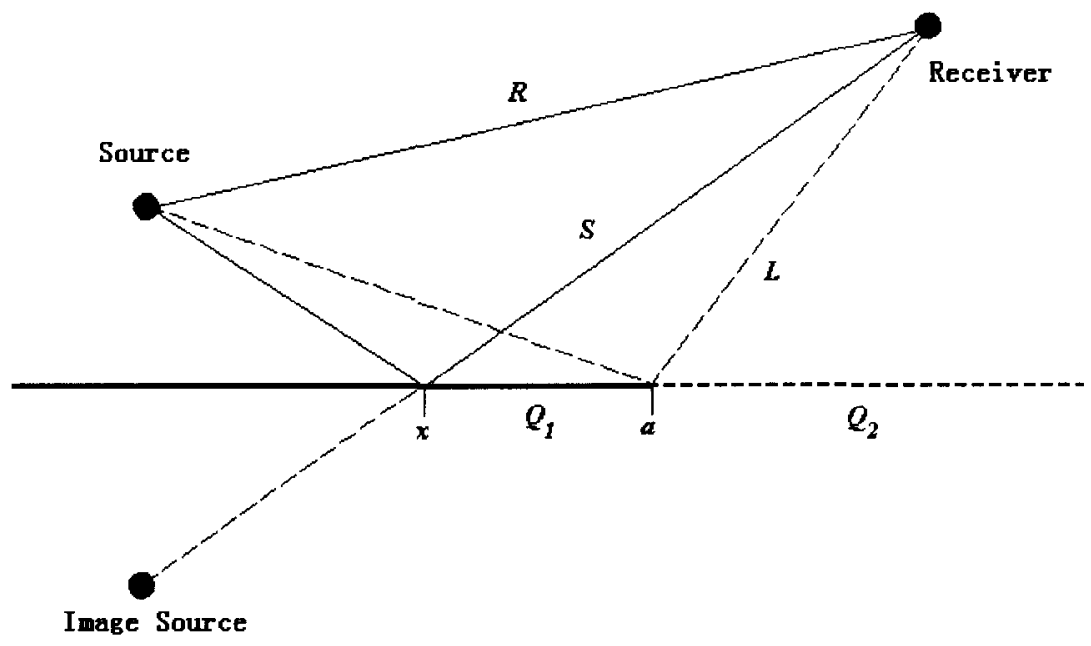

Figure 4-15. Geometry of diffraction at a screen (De Jong, 1983)

In the 1970s, extensive measurements of highway traffic noise were carried out; however, it was hard to establish a connection between the theoretical results and the measured results. One of the reasons is that the influence of the hard road surface has often been neglected. However, this neglect will lead to errors of some magnitude (Attenborough, 1982). In the following section, several models dealing with diffraction at an impedance discontinuity will be discussed.

In this section, a heuristic semi-empirical solution derived by De Jong for such a problem will be presented. This model is based on an expression for the diffraction by a plane hard screen, assumed to be located in the ground. Another model developed by Rasmussen was based on Kirchhoff-Rayleigh's approximation to the sound field over the transition line between the two impedance values of the ground. This model was more accurate compared with De Jong's model but also more complex and was time consuming for numerical computation. To evaluate the proportions of impedance of each ground type contributing significantly to the excess attenuation at the receiver point, 
Horthersall and Harriott (1995) developed the Fresnel-zone model, which is a rather straightforward method. This method was later improved by Birger Plovsing to give a better result in the high frequency region.

\subsubsection{De Jong's Impedance Model (De Jong, 1983)}

Suppose we have two semi-infinite planes having admittances $\beta_{1}$ and $\beta_{2}$, and reflection coefficients $Q_{1}$ and $Q_{2}$. The total sound field at the receiver can be described as a component of geometrical acoustics for a direct and reflected term $p_{i}+Q_{2} p_{r}$ (for $x>a)$, and a diffraction term, although the exact formula for this term at the impedance discontinuity is still unknown; hence:

$$
p_{i}=p_{i}+Q_{2} p_{r}+p_{d i f f}^{\prime}
$$

To construct the solution for $p_{d i f f}^{\prime}$, we made an extrapolation for the known special case of a semi-infinite screen, which can be regarded as an impedance step from an acoustically hard surface to air. Shown as Figure 4-15, the total sound field can be given as:

$$
\begin{aligned}
p_{t}=p_{i}\left(1-D_{i}\right)+Q_{1} p_{r}-D_{r} p_{r} & \text { for } x<a \\
p_{t}=p_{i}\left(1-D_{i}\right)+D_{r} p_{r} & \text { for } x>a
\end{aligned}
$$

Since the solution is continuous for all $x, D_{r}$ should equal $1 / 2$ when $x=a$.

For the original problem, we construct a formula of total sound field, so that $p_{\text {diff }}$ in Equation (4-47) can be split into two diffraction terms as follows:

$$
\begin{aligned}
& p_{t}=p_{i}\left(1-D_{i}^{\prime}\right)+Q_{1} p_{r}-D_{r}^{\prime} p_{r} \text { for } x<a \\
& p_{t}=p_{i}\left(1-D_{i}^{\prime}\right)+Q_{2} p_{r}+D_{r}^{\prime} p_{r} \text { for } x>a
\end{aligned}
$$


When the reflection point is exactly at the impedance discontinuity $(x=a)$, Equation (6) in Ingard's paper can be changed into an integration from 0 to $\pi$ with $\beta=\beta_{1}$, and an integration from $\pi$ to $2 \pi$ with $\beta=\beta_{2}$. So the total sound field can be written as follows:

$$
p_{t}=p_{i}+Q_{t o t} p_{r}=p_{i}+\frac{1}{2}\left(Q_{1}+Q_{2}\right) p_{r} \quad \text { for } x=a
$$

Comparing Equation (4-52) and (4-48), (4-52) and (4-49), they should be the same equation with $D_{r}=1 / 2$ when $Q_{1}=1$ and $Q_{2}=0$. However, this is not true since in most of the cases $D_{i} \neq 0$. Obviously the diffraction term is lack in Equation (4-52). By incorporating a diffraction term of the direct field, the total sound field can be described as follows:

$$
p_{t}=p_{i}\left(1-D_{i}^{\prime}\right)+\frac{1}{2}\left(Q_{1}+Q_{2}\right) p_{r} \text { for } x=a
$$

Equation (4-53), (4-48) and (4-49) agree with each other, since Equation (4-53), (4-50), and (4-51) should be of the same form when requiring continuity at $x=a$, which yields

$$
D_{r}^{\prime}=\frac{1}{2}\left(Q_{1}-Q_{2}\right) \text { for } x=a
$$

So the most plausible solution for diffraction coefficient $D_{r}^{\prime}$ when considering each point along $\mathrm{x}$-axis is as follows:

$$
D_{r}^{\prime}=\left(Q_{1}-Q_{2}\right) D_{r} \text { for all } x
$$

Similarly, diffraction coefficient $D_{i}^{\prime}$ can be deducted in the same way.

$$
D_{i}^{\prime}=\left(Q_{1}-Q_{2}\right) D_{i} \text { for all } x
$$


As a summary, when considering sound propagation over different ground types with impedance discontinuity, the De Jong's impedance model described above leads to the following solution for the total sound field:

$$
\begin{gathered}
p_{t}=p_{i}-\left(1-Q_{\text {soft }}\right) D_{i} p_{i}+Q_{\text {hard }} p_{r}-\left(1-Q_{\text {soft }}\right) D_{r} p_{r} \text { for } x<a \\
p_{t}=p_{i}-\left(1-Q_{\text {sofit }}\right) D_{i} p_{i}+Q_{\text {sfor }} p_{r}+\left(1-Q_{\text {soff }}\right) D_{r} p_{r} \text { for } x>a
\end{gathered}
$$

where,

$Q_{\text {soft }}$ the spherical reflection coefficient on the soft ground surface, such as grassland or lawn

$Q_{\text {hard }}$ the spherical reflection coefficient on the hard ground surface, such as asphalt or concrete pavement

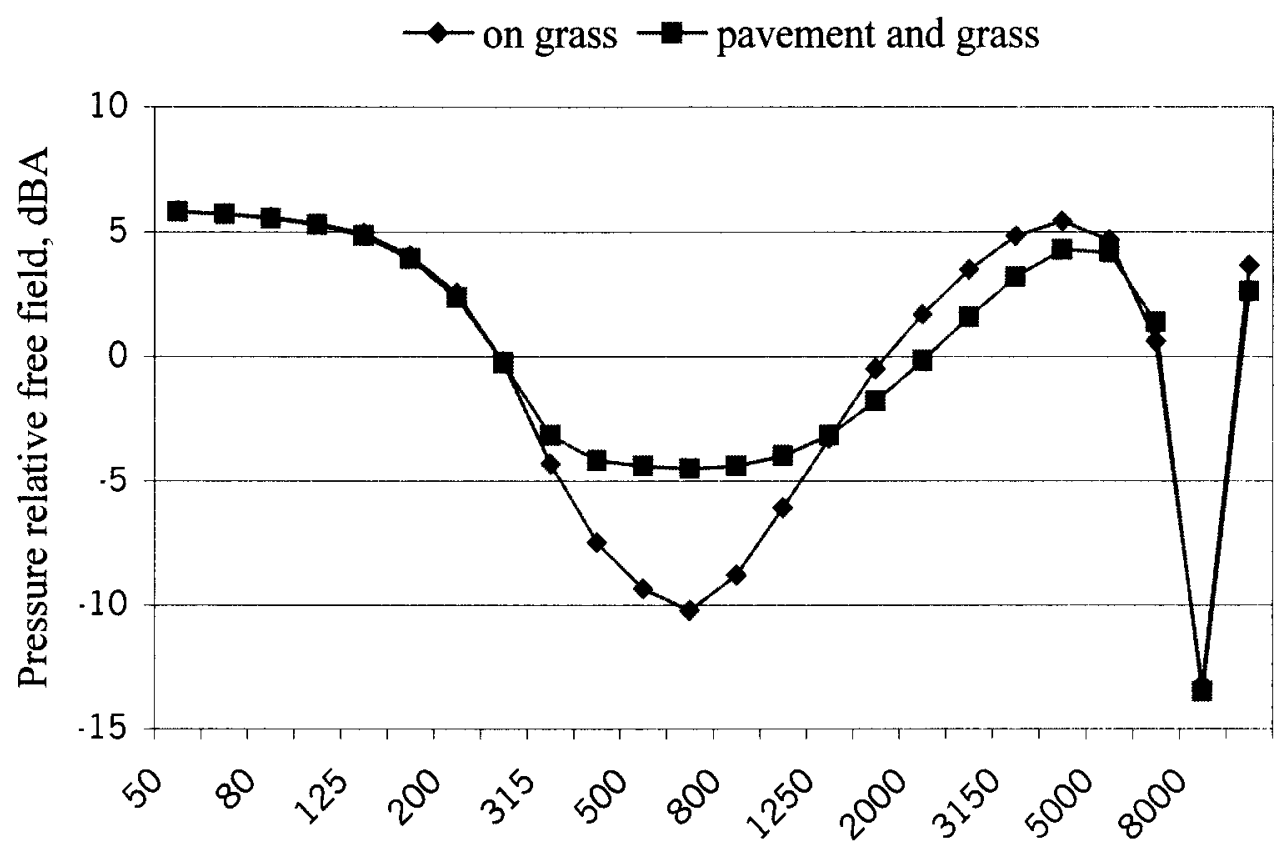

Frequency, $\mathrm{Hz}$

Figure 4-16. De Jong's model compared to ignoring the hard surface

Figure 4-16 shows the excess attenuation of De Jong's model compared to that of ignoring the hard surface, with $E F R_{1}=20,000 \mathrm{cgs}$ Rayls, $E F R_{2}=325 \mathrm{cgs}$ Rayls, 
corresponding to an asphalt and a grass surface, $h_{s}=h_{r}=1.5 \mathrm{~m}(5 \mathrm{ft}), d=100 \mathrm{~m}(328$ $\mathrm{ft})$, discontinuity point $40 \mathrm{~m}(131 \mathrm{ft})$ from the source.

De Jong's impedance model is a semi-empirical model based on physical arguments and intuition. It works quite well in many cases, but not in all cases. As illustrated in Fig 4-17 by Ögren (1997), De Jong's model gives questionable results compared to the results of ignoring the soft surface, and to PROPAG program, which uses a numerical method developed by Rasmussen.

Boulanger (1997) pointed out that De Jong's impedance model predicted excess attenuation poorly in the case of multiple impedance discontinuities, where the depth of the first dip is over-predicted by more than $20 \mathrm{dBA}$.

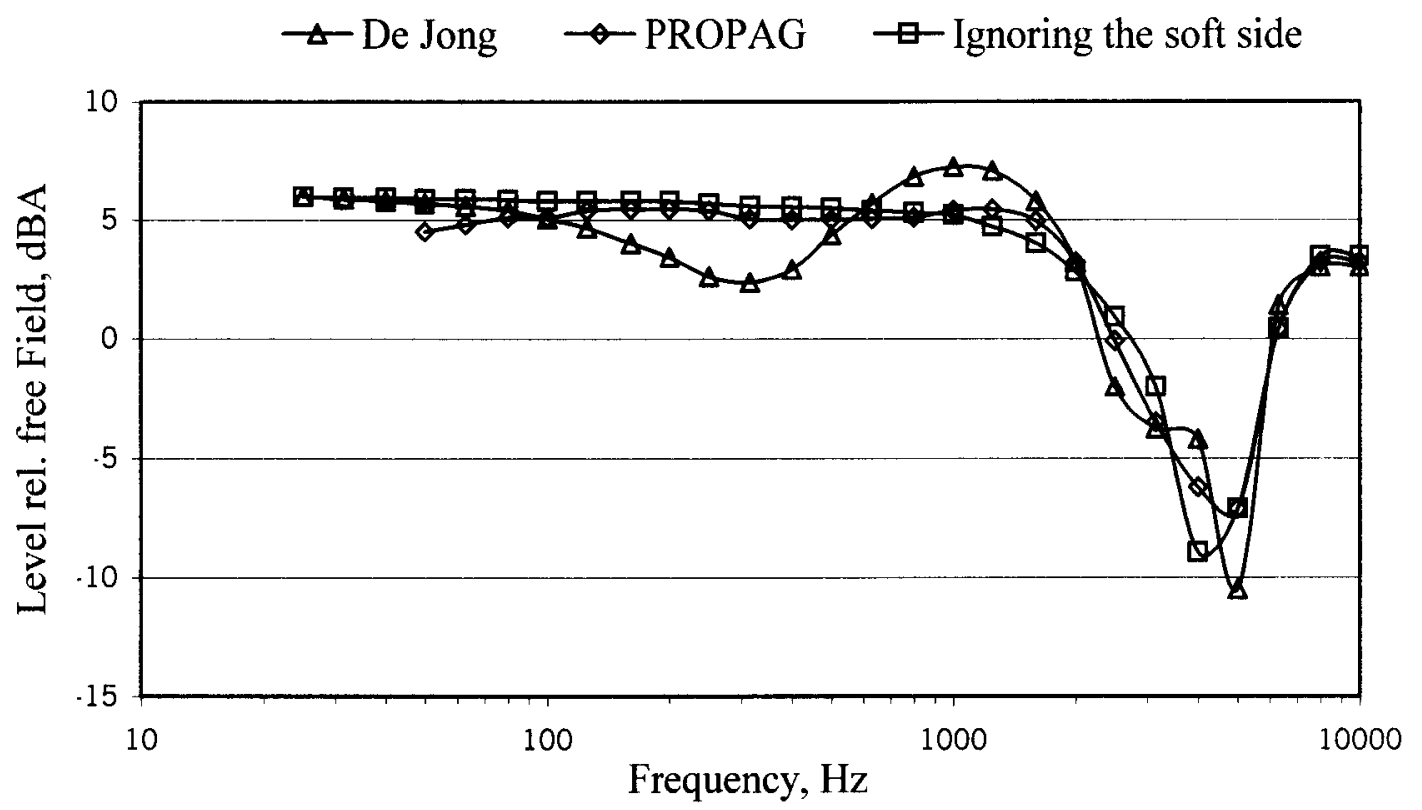

Figure 4-17. De Jong compared to ignoring the soft surface, and to the PROPAG from Delta (Ögren, 1997) 


\subsubsection{The Fresnel-Zone Model}

In this session, a brief introduction of the Fresnel-zone model will be given.

Figure 4-18 shows the definition of symbols for the Fresnel-zone model. In principle this model uses equations as follows:

$$
20 \log \left(\left|p_{T}\right|\right)=r 20 \log \left(\left|p_{s}\right|\right)+(1-r) 20 \log \left(\left|p_{R}\right|\right)
$$

where,

$p_{T} \quad$ the solution above the plane with the impedance discontinuity

$p_{s} \quad$ the solution with the source side impedance over the whole surface

$p_{r} \quad$ the solution with the receiver side impedance over the whole surface

$r \quad$ the ratio of $x_{1}$ (if $x_{1}>0$ ) and the total length of the Fresnel zone

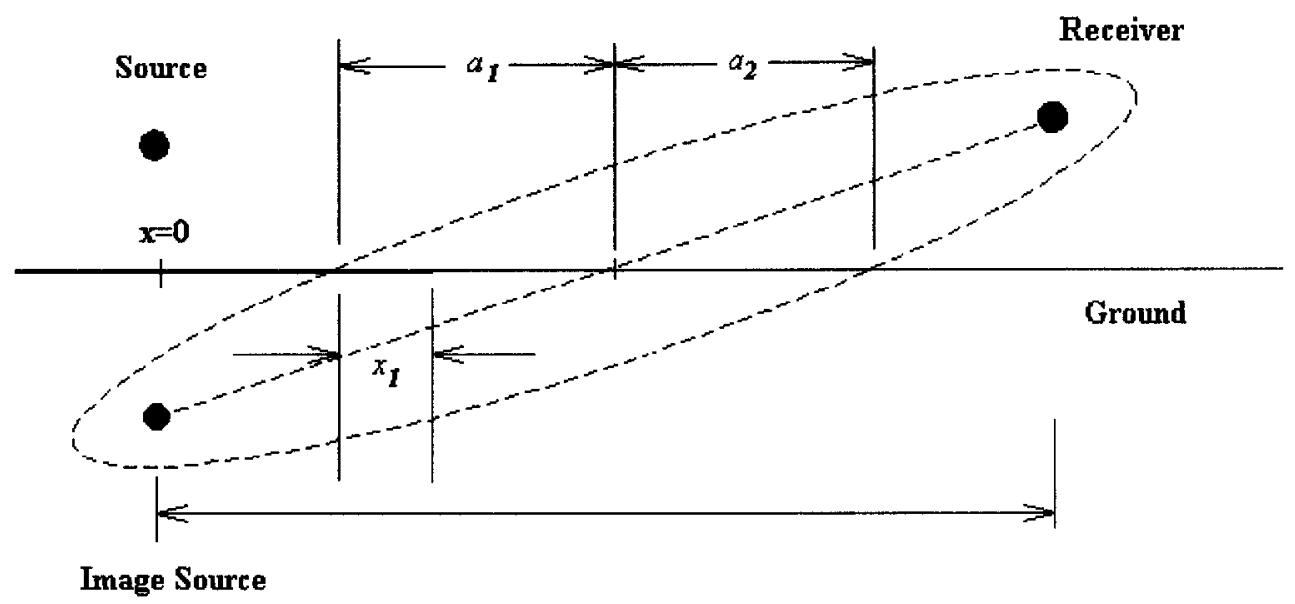

Figure 4-18. Definition of symbols for the Fresnel zone model (Ögren, 1997)

$r$ is defined as follows: 


$$
r= \begin{cases}\frac{x_{1}}{a_{1}+a_{2}} & 0<x_{1} \leq a_{1}+a_{2} \\ 1 & x_{1}>a_{1}+a_{2} \\ 0 & x_{1} \leq 0\end{cases}
$$

From Equation (4-60), we can see that if $x_{1}$ is less than 0 , then $r=0$, the total sound field will totally depend on the receiver side impedance. If $x_{1}$ is greater than $a_{1}+a_{2}$, then $r=1$, only source side impedance will affect the total sound field.

By making an ellipse around the image source and the receiver, the Fresnel zone can be determined. The horizontal intersection distance of the ellipse with the ground, $a_{1}$, $a_{2}$, can be given as follows after some approximations:

$$
\begin{aligned}
& a_{1}=\frac{d}{2} \frac{\frac{h_{s}-h_{r}}{h_{s}+h_{r}}+\sqrt{1+\frac{2 h_{s} h_{r}}{C \lambda d}}}{1+\frac{\left(h_{s}+h_{r}\right)^{2}}{2 C \lambda d}} \\
& a_{2}=\frac{d}{2} \frac{\frac{h_{r}-h_{s}}{h_{s}+h_{r}}+\sqrt{1+\frac{2 h_{s} h_{r}}{C \lambda d}}}{1+\frac{\left(h_{s}+h_{r}\right)^{2}}{2 C \lambda d}}
\end{aligned}
$$

where,

$h_{s} \quad$ source height

$h_{r} \quad$ receiver height

$d$ the horizontal distance between source and receiver

C $\quad 1 / 4$ is recommended

De Jong's impedance model predicted results similar to those of the Fresnel-zone 
model in some cases, as shown in Figure 4-19. But this is not true for all cases. Figure 420 shows that De Jong's impedance model will not only change the amplitude of ground attenuation, but also change the frequency position of the interference dip. The Fresnelzone model just changes the amplitude of the ground attenuation. The interference dip is at the same frequency as that of ignoring the hard surface.

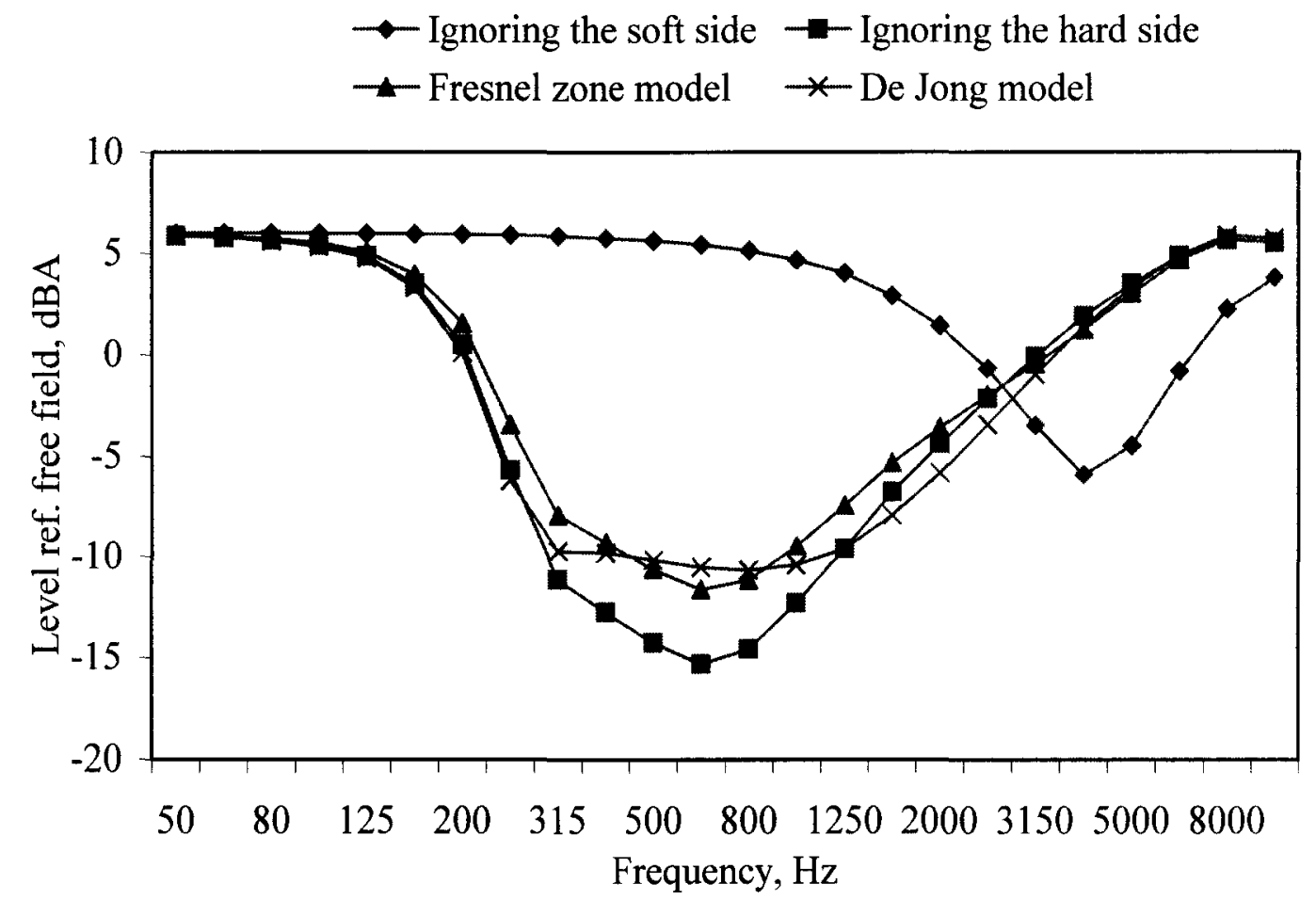

Figure 4-19. Fresnel-zone model compared to De Jong's model, distance $=100 \mathrm{~m}$, impedance discontinuity at $20 \mathrm{~m}, h_{r}=h_{s}=1 \mathrm{~m}$ 


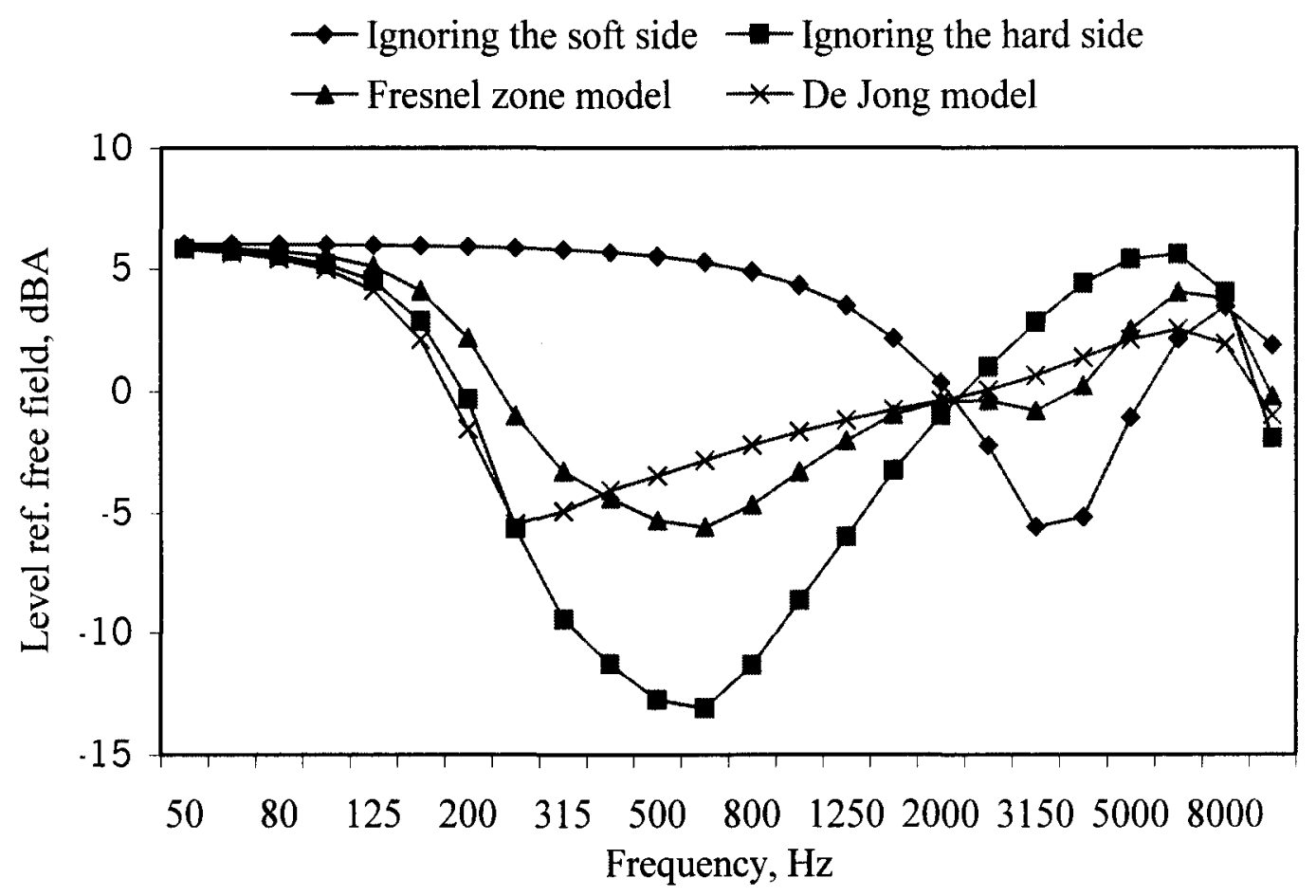

Figure 4-20. Fresnel-Zone model compared to De Jong's model, distance $=100 \mathrm{~m}$, impedance discontinuity at $50 \mathrm{~m}, h_{r}=h_{s}=1.5 \mathrm{~m}$

\subsubsection{Rasmussen's Impedance Model}

Rasmussen (1982) developed a direct approach to sound propagation over an impedance discontinuity, which was based on the ideas employed by Thomasson (1978) with Rayleigh approximations. This model assumes that the sound field may be regarded as consisting of two parts, the first part being calculated as sound propagation over infinite plane with impedance $Z_{1}$, and the second part being calculated as sound propagation over infinite plane with impedance $Z_{2}$, shown in Figure 4-21. $R_{1}, R_{2}, R_{3}$, and $R_{4}$ denote the ray paths located in the vertical plane containing source and receiver. $Q_{1}$ and $Q_{2}$ denote the reflection coefficients on the source side and the receiver side. The total sound field is calculated by joining the two parts over the impedance discontinuity; the total velocity potential is given as follows: 


$$
\phi=2 \int_{0}^{\infty} \int_{0}^{\infty}\left[\frac{e^{i k R_{1}}}{R_{1}}+Q_{1} \frac{e^{i k R_{2}}}{R_{2}}\right] \times\left[\left(-i k+1 / R_{3}\right) \frac{e^{i k R_{3}}}{R_{3}^{2}} x_{R}+\left(-i k+1 / R_{4}\right) Q_{2} \frac{e^{i k R_{4}}}{R_{4}^{2}} x_{R}\right] d y d z
$$

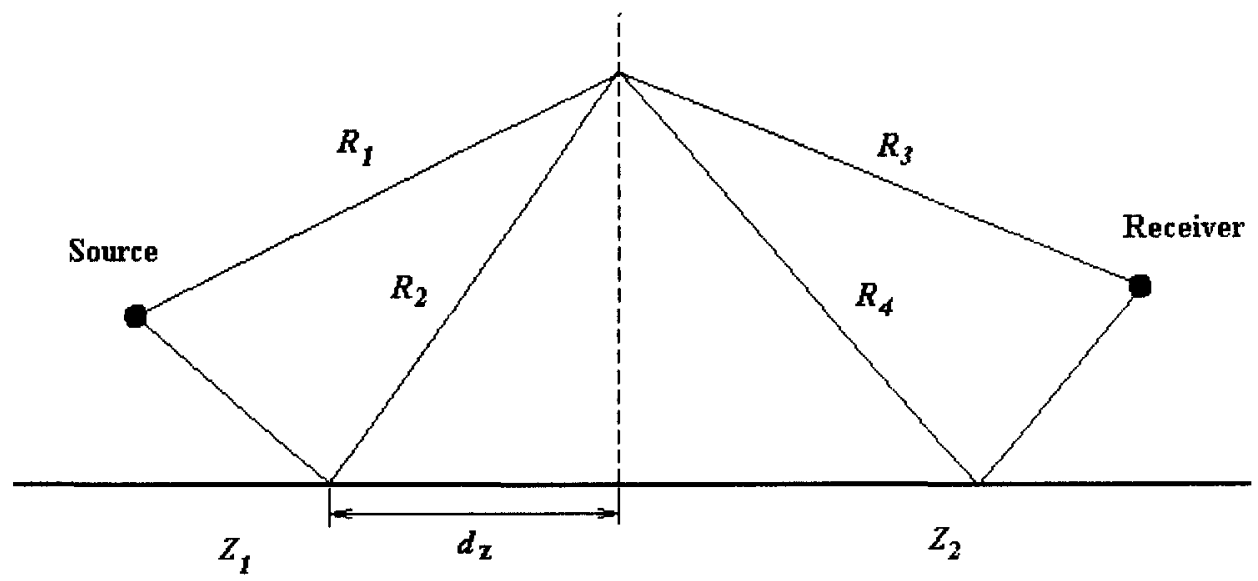

Figure 4-21. Step-wise calculation of sound field (Rasmussen, 1982)

Rasmussen also developed a numerical method for the possible numerical computation. This was done by means of 20,000 points located over the discontinuity and covering an area of $40 \lambda$ wide and $20 \lambda$ tall. For each point, $Q_{1}$ and $Q_{2}$ needs to be calculated once. He showed that his model agreed well with measurement data and even better than De Jong's impedance model. However, this model needs much more computer time than De Jong's impedance model.

\subsection{Empirical Formulas}

Many researchers carried out extensive studies on acoustical performance of a noise barrier through indoor scale-model experiments. Indoor scale-model experiments have many advantages when compared with full-scale field measurements. They have low cost and are free of weather influence. The first known graph for barrier attenuation, 
due to a point source in a shadow zone, was developed by Redfearn (1940). Two parameters were used in his graph, namely, the effective height of barriers normalized by the wavelength and the angle of diffraction. In 1968, Maekawa (1968) developed his famous design chart for a thin, rigid barrier based on measurements of extensive configuration of source and receiver locations. The barrier attenuation was given as a function of a single Fresnel number. In 1971 Keller derived his equation for barrier attenuation based on the geometrical acoustics; however, his theory is an asymptotic expansion valid in the far field. Hence, calculated results deviate from precise values at low frequencies or low Fresnel numbers. Kurze and Anderson (1971) reviewed diffraction theory from Keller, and utilized Maekawa's and Redfearn's experimental data to develop his empirical formulas for barrier attenuation. Kawai et al. (1978) developed a simple, approximate expression from Bowman and Senior's formula using the Fresnel number, which was based on Macdonald's rigorous solution. Kawai also discussed the effect of the image source in a perfectly reflecting barrier using the second term of the approximate expression of Macdonald's solution. Li et al. (2005) obtained comprehensive data based on their own measurements at the anechoic room in order to determine the barrier attenuation relative to free field. This free field level is the standard reference for outdoor sound propagation. Different geometrical configurations were used with varying frequency ranges from $500 \mathrm{~Hz}$ to $10 \mathrm{KHz}$. Their experimental data were consistent with the data in Maekawa's chart. Accurate analytical solutions and empirical formulas were also compared with his measurement data. Due to their simplicity, the Kurze-Anderson formula and Maekawa's design chart have been used extensively for practical engineering. 
In the following section, Maekawa's design chart will be discussed in more detail. In Maekawa's measurements, receivers were located not only in the shadow zone but also in the illuminated zones. In the shadow zone, the line of sight from the source point to receiver was blocked by the barrier and the Fresnel number became a positive value. In the illuminated zone, the source point can see the receiver and the Fresnel number became a negative value. When the receiver is in the transition point from the shadow zone to illumination zone, the attenuation is $5 \mathrm{dBA}$ and Fresnel number is 0 according to Maekawa's design chart.

A simple function of barrier attenuation that fits the Maekawa data quite well is as follows:

$$
I L=10 \log \left(3+20 N_{1}\right)
$$

The above formula is originally defined for $N_{1}>0$, but is often used for $N_{1}>-0.05$, where the illuminated zone is also considered.

The simple formula derived by Kurze and Anderson (1971) has been used widely based on the Maekawa's data, shown as follows:

$$
I L=5+20 \log \frac{\sqrt{2 \pi N_{1}}}{\tanh \sqrt{2 \pi N_{1}}} \quad \text { for } \quad \mathrm{N}_{1}>0 .
$$

Maekawa's results indicated that Fresnel-Kirchhoff's theory over-predicted the noise attenuation by a few dBA than those of Redfearn's and Maekawa's curve (Maekawa, 1965). Maekawa's curve was in close agreement with those of Redfearn's, but in a simple representation form.

The extensive experiments based on the scale model by $\mathrm{Li}$ (2005) also demonstrated MacDonald's solution predicted more accurate insertion loss than 
Maekawa's chart, which under-predicted the insertion loss for Fresnel number ranged from 0.1 to 10, as shown in Figure 4-22. As a matter of fact, Kurze-Anderson's formula has additional under-prediction of insertion loss within $1 \mathrm{dBA}$ compared to Maekawa's chart. Furthermore, Li demonstrated that MacDonald's solution better predicted insertion loss than Fresnel-Kirchhoff's approximation, which over-predicted the insertion loss compared with measurement data.

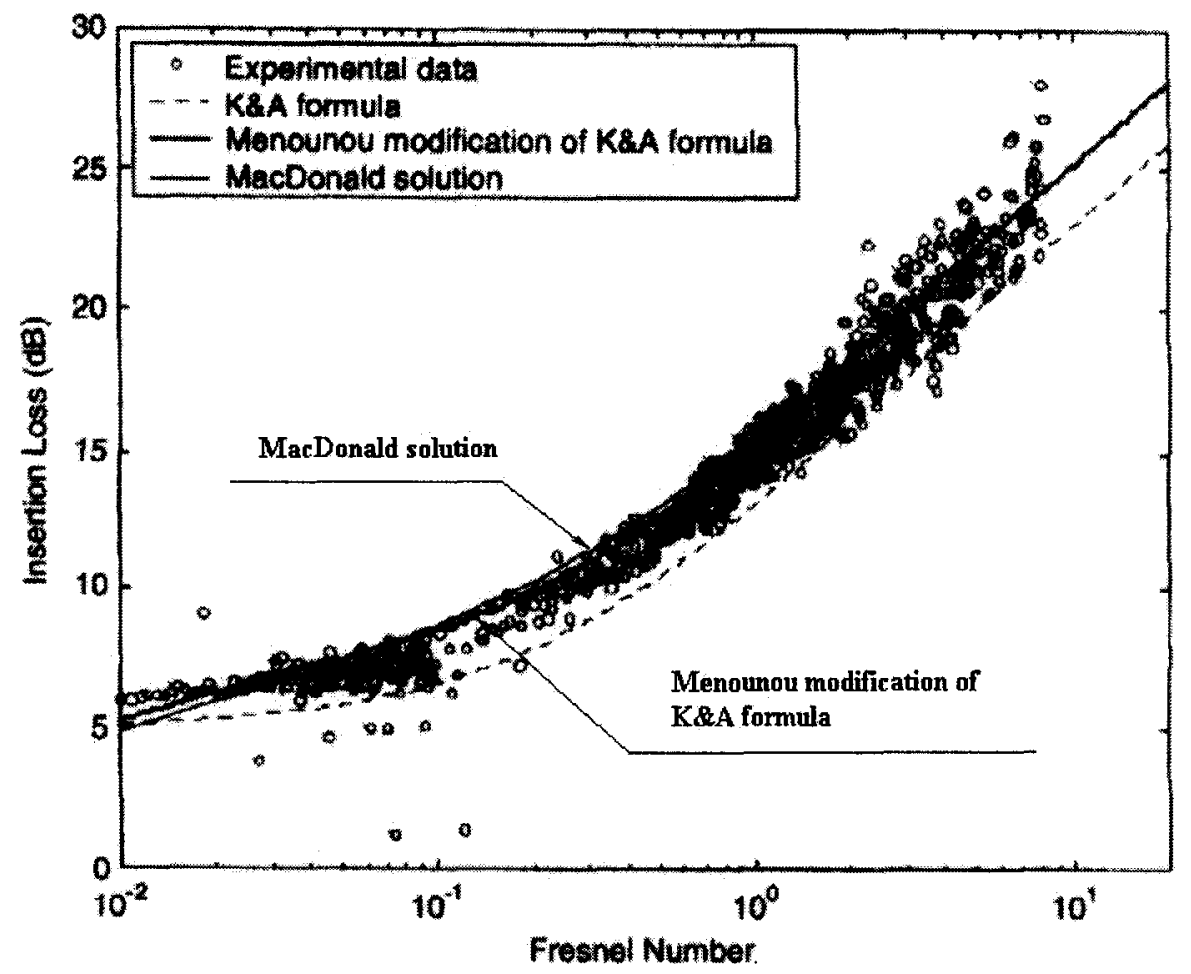

Figure 4-22. Comparison of the Kurze-Anderson's formula with experimental data ( $\mathrm{Li}$, 2005)

In 1992, Yamamoto and Takagi (1992) proposed more accurate formulas with four different types of approximate expressions, based on the Maekawa's design chart. Their formulas are as follows:

$$
I L= \begin{cases}10 \log \left[1+G\left(N_{1}\right)\left(N_{1}+0.3\right)\right] & \text { for } N_{1}>-0.3, \\ 0 & \text { for } N_{1}<-0.3\end{cases}
$$


where,

$$
G\left(N_{1}\right)=3.621\left\{\tan \left(\frac{N_{1}-5 \times 10^{-3}}{1.45 \times 10^{-2}}\right)+\frac{\pi}{2}\right\}+6.165\left\{1-e^{-0.205\left(N_{1}+0.3\right)}\right\}+2.354
$$

(ii)

$$
I L= \begin{cases}10 \log N_{1}+13 & \text { for } N_{1}>1 \\ 5+8 N_{1} /\left|N_{1}\right|^{0.55+0.143\left|N_{1}\right|} & \text { for }-0.3<\mathrm{N}_{1}<1 \\ 0 & \text { for } N_{1}<-0.3\end{cases}
$$

(iii)

$$
I L= \begin{cases}10 \log N_{1}+13 & \text { for } N_{1}>1 \\ 5 \pm 8\left|N_{1}\right|^{0.438} & \text { for }-0.3<N_{1}<1 \\ 0 & \text { for } N_{1}<-0.3 .\end{cases}
$$

(iv)

$$
I L= \begin{cases}10 \log N_{1}+13 & \text { for } N_{1}>1 \\ 5 \pm 9.07674 \times \sinh ^{-1}\left|N_{1}\right|^{0.485} & \text { for }-0.3<N_{1}<1 \\ 0 & \text { for } \quad N_{1}<-0.3\end{cases}
$$

Formulas (i) - (iv) closely agreed with the data from Maekawa's design chart with a maximum difference of less than $0.5 \mathrm{dBA}$ in (i) and is no more than $0.3 \mathrm{dBA}$ in cases (ii) - (iv).

Menounou (2000) improved Kurze-Anderson's empirical formula by introducing two Fresnel numbers. The first Fresnel number, $N_{1}$, is the conventional Fresnel number, which is associated with the relative position of the source to the barrier and the receiver. The second Fresnel number, $N_{2}$, denoted to the relative position of the image source to the barrier and the receiver. The improved Kurze-Anderson's formula is given by:

$$
I L=I L_{s}+I L_{b}+I L_{s b}+I L_{s p}
$$

where, 


$$
\begin{gathered}
I L_{s}=20 \log \frac{\sqrt{2 \pi N_{1}}}{\tanh \sqrt{2 \pi N_{1}}}-1 \\
I L_{b}=20 \log \left[1+\tanh \left(0.6 \log \frac{N_{2}}{N_{1}}\right)\right], \\
I L_{s h}=\left(6 \tanh \sqrt{N_{2}}-2-A t t_{b}\right)\left(1-\tanh \sqrt{10 N_{1}}\right), \\
I L_{s p}=-10 \log \frac{1}{\left(R^{\prime} / R_{1}\right)^{2}+\left(R^{\prime} / R_{1}\right)}
\end{gathered}
$$

\subsection{Jonasson's Four-Path Model}

By combining the effect of noise barrier and ground surfaces, Jonasson developed a four-path model to calculate noise levels at receivers, as shown in Figure 4-23. Suppose the spherical coefficient on the ground surface of the source side is $Q_{1}$ and $Q_{2}$ on the receiver side, the total sound pressure in the shadow zone behind the noise barrier is written as:

$$
p_{t}=p_{\text {diff }, 11}+Q_{1} p_{\text {diff }, 21}+Q_{2} p_{\text {diff }, 12}+Q_{1} Q_{2} p_{d i f f, 22}
$$

where,

$p_{\text {diff }, 11} \quad$ the diffracted sound pressure with propagation path from the source to the receiver

$p_{\text {diff }, 21} \quad$ the diffracted sound pressure with propagation path from image source to the receiver

$p_{\text {diff }, 12}$ the diffracted sound pressure with propagation path from the source to the image receiver

$p_{\text {diff }, 22}$ the diffracted sound pressure with propagation path from the image source to the image receiver 
For each propagation path, the diffracted sound pressure is calculated with the equations as:

$$
p_{d i f f, n m}=D_{i} \frac{e^{i k R_{n m}}}{k R_{n m}}+D_{r} \frac{e^{i k S_{n m}}}{k S_{n m}}, \quad \text { with }\left\{\begin{array}{l}
n=1,2 \\
m=1,2
\end{array}\right\}
$$

where,

$D_{i}$ and $D_{r}$ defined by Equations (4-29) and (4-30)

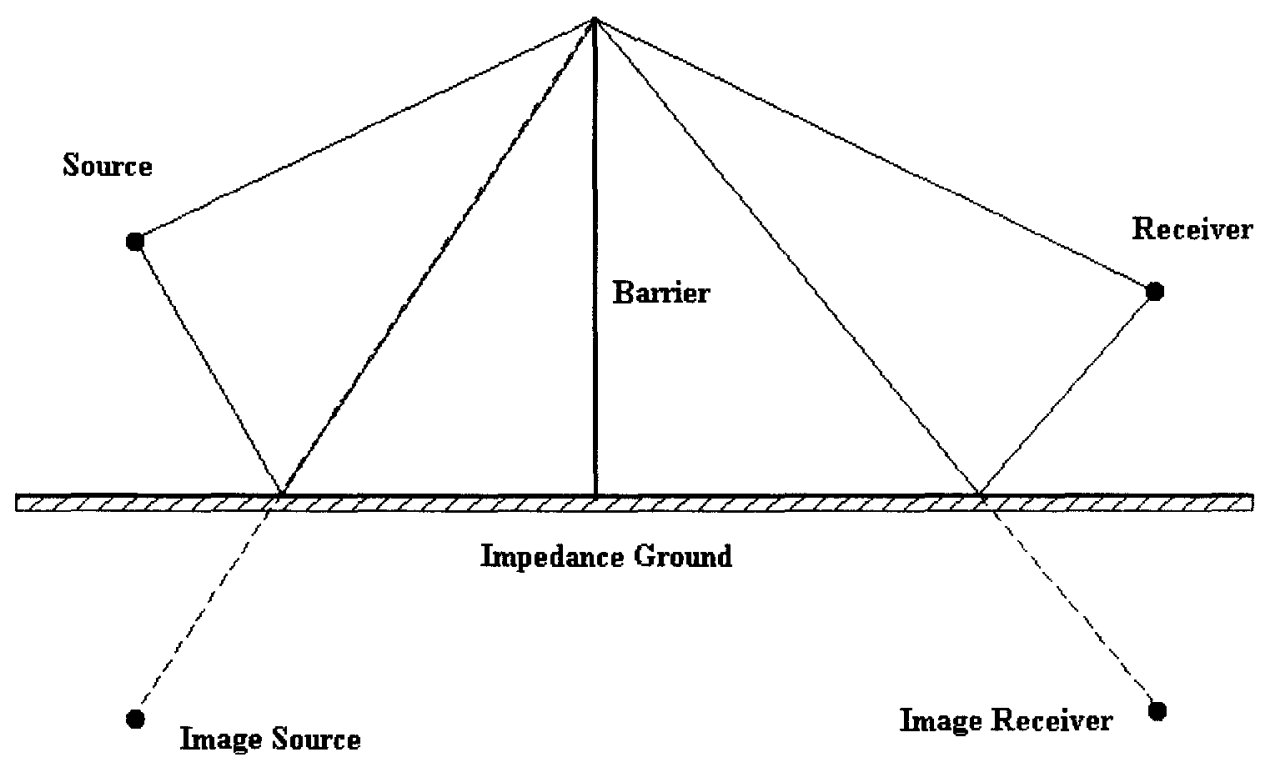

Figure 4-23. The four propagation paths for Janasson's model (De Jong, 1983)

\subsection{Diffraction Theory in STAMINA 2.0}

The attenuation provided by a barrier in STAMINA 2.0 can be expressed as a function of the Fresnel number, the barrier shape, and the barrier length in the following form:

$$
\Delta_{B_{i}}=10 \log \left[\frac{1}{\phi_{R}-\phi_{I_{l}}} \int_{\phi_{l}}^{\phi_{R}} 10^{\frac{-\Delta_{i}}{10}} d \phi\right]
$$

where, 
$\Delta_{B_{l}} \quad$ the attenuation provided by the barrier for the $\mathrm{i}^{\text {th }}$ class of vehicles.

$\phi_{R}, \phi_{l}$ the angles that establish the relationship (position) between the barrier and the observer.

$$
\Delta_{i}= \begin{cases}0 & N_{i} \leq-0.1916-0.0635 \varepsilon \\ 5(1+0.6 \varepsilon)+20 \log \frac{\sqrt{2 \pi\left|N_{o}\right|_{i} \cos \phi}}{\tan \sqrt{2 \pi\left|N_{0}\right|_{i} \cos \phi}} & (-0.1916-0.0635 \varepsilon) \leq N_{i} \leq 0 \\ 5(1+0.6 \varepsilon)+20 \log \frac{\sqrt{2 \pi\left(N_{0}\right)_{i} \cos \phi}}{\tanh \sqrt{2 \pi\left(N_{0}\right)_{i} \cos \phi}} & 0 \leq N_{i} \leq 5.03 \\ 20(1+0.15 \varepsilon) & N_{i} \geq 5.03\end{cases}
$$

where,

$\Delta_{i} \quad$ the point source attenuation for the $\mathrm{i}^{\text {th }}$ class of vehicles

$$
N_{i}=\left(N_{0}\right)_{i} \cos \phi
$$

$\varepsilon \quad$ a barrier shape parameter, 0 for a freestanding wall and 1 for an earth berm

$N_{0} \quad$ the Fresnel number determined along the perpendicular line between the source and receiver

$N_{0 i} \quad$ the Fresnel number of the $\mathrm{i}^{\text {th }}$ class of vehicles determined along the perpendicular line between the source and receiver

Mathematically the Fresnel number, $N_{0}$, is defined as

$$
N_{0}=2\left(\frac{\delta_{0}}{\lambda}\right)
$$

where,

$\delta_{0} \quad$ the path length difference measured along the perpendicular line between the source and receiver

$\lambda \quad$ the wavelength of the sound radiated by the source 
The path length difference, $\delta_{o}$, is the difference between a perpendicular ray traveling directly to the observer and a ray diffracted over the top of the barrier:

$$
\delta_{o}=A_{o}+B_{o}-C_{o}
$$

If the height of the noise source or the observer changes, the path length difference will also change.

For barrier calculations only, the vehicle noise sources are assumed to be located at the following positions:

(1) Automobiles: $\quad 0$ meters above the centerline of the lane

(2) Medium Trucks: 0.7 meters above the centerline of the lane

(3) Heavy Trucks: 2.44 meters above the centerline of the lane

\subsection{Diffraction Theory and Propagation Theory in TNM 2.5}

\subsubsection{Propagation Path Calculations and Mathematical Description}

The mathematical model used to calculate the attenuation due to the vertical geometry between the source and the receiver was in large part developed from work by De Jong (1983), Chessell (1977), Delany (1970), Boulanger (1997) and Foss (1976). The diffraction term for TNM 2.5 is defined as follows:

$$
D=\frac{R}{L} \frac{e^{-i \frac{\pi}{4}}}{\sqrt{\pi}} e^{i k(L-R)} e^{-i \chi^{2}} F(\chi)
$$

where,

$L$ is defined as the propagation path length. $D$ is multiplied by a sign function that is positive when the receiver is in the shadow zone and negative when the receiver is in the illuminated zone. TNM 2.5 uses an adjustment factor, $A$, to adjust the diffraction 
field to make it consistent with empirical results, $A$ is currently set to 1.2 . The factor $Q$ is included to account for the surface impedances at the diffracting edge. This results in the following equation:

$$
D=(\operatorname{sgn}) A D Q
$$

The $\chi$ function has the following equation:

$$
\chi=\left(\frac{k r r_{0}}{2 L}\right)^{1 / 2}\left|\frac{\cos (\pi / v)-\cos \left(\left(\phi-\phi_{0}\right) / v\right)}{(1 / v) \sin (\pi / v)}\right|
$$

where,

$$
v=\frac{(2 \pi-T)}{\pi}
$$

$T$ is the positive "top angle" of the wedge ( $T=0$ for a barrier, and $T=\pi$ for a flat surface). For diffracting edges like barriers, where the interior wedge angle, $T$, is 0 , $\chi$ can be simplified to the following equation:

$$
\chi=\sqrt{\frac{k(L-R)(L+R)}{2 L}}
$$

\subsubsection{Single Diffraction}

The following equation is used to calculate the sound pressure for a propagation path with a single diffraction, assuming that material at the diffraction point is acoustically hard:

$$
P_{\text {path }}=p_{\text {free-field }} D
$$

If the material at the diffraction point is other than acoustically hard, $D$ is multiplied by the reflection coefficient, $Q$. 


\subsubsection{Reflection Coefficients}

Coefficients of reflection $Q$ are calculated with Chessell's model (1977). This model takes frequency, effective flow resistivity (EFR) of the reflecting segment, and the geometry configurations into account. $Q$ is computed according to the following equation:

$$
Q=R_{p}+F(w)\left(1-R_{p}\right)
$$

where $R_{p}$ is the term for the incident wave and is calculated with the following equation:

$$
R_{p}=\frac{\sin \phi-\frac{Z_{0}}{Z}}{\sin \phi+\frac{Z_{0}}{Z}}
$$

where,

$Z_{0} \quad$ the impedance of air

$\varphi \quad$ the angle of incidence of the propagation path on the reflecting segment

$Z \quad$ the acoustic impedance of the reflection surface

$$
Z=\left[1+.051\left(\frac{f}{\sigma}\right)^{-.75}+0.77\left(\frac{f}{\sigma}\right)^{-.73} i\right] Z_{0}
$$

where,

$\sigma \quad$ the effective flow resistivity (EFR) for the reflecting segment in MKS Rayls, MKS unit is based on meter, kilogram and second system

$F(w)$ the ground wave function. It is defined by the following equation: 


$$
F(w)=\left[\begin{array}{cc}
1+i e^{-w} \sqrt{\pi w}-2 e^{-w} \sum_{n=1}^{\infty} \frac{w^{n}}{(n-1) !(2 n-1)} & |w|<10 \\
-\sum_{n=1}^{\infty} \frac{(2 n) !}{2^{n} n !(2 w)^{n}} & |w| \geq 10
\end{array}\right]
$$

where,

$w \quad$ the numerical distance, is defined as follows:

$$
w=\frac{1}{2} i k r \frac{\left(\sin \phi+Z_{0} / Z\right)^{2}}{1+\sin \phi Z_{0} / Z}
$$

where,

$k \quad$ the wave number

$r \quad$ the total distance between the source and the receiver through the medium.

However, according to other original papers (Chessell, 1977), (Jonasson, 1972), $r^{\prime}$, the total distance between the image source and the receiver, should be used instead of $r$.

\subsubsection{Reflected Path}

The reflection coefficient $Q$ is multiplied with the propagation path to account for energy loss and phase shift due to the reflection. A propagation path is multiplied by one coefficient for each segment that reflects the path. As pointed out by Menge (1996), TNM 1.0 does not account for the reflection point on the receiver side. TNM does not change this feature in later versions according to the TNM Technical Manual (Menge et al., 1998). That means TNM 2.5 reduces four propagation paths by Janasson's model to two propagation paths model. This modification has not been validated before and may result in inaccuracy in some cases. Chapter VII will discuss this problem in more detail. 


$$
P_{\text {path }}=p_{\text {free-field }} Q D
$$

TNM 2.5 applies De Jong's (1983) impedance model to compute the impedance discontinuities. Propagation paths with diffractions at impedance discontinuities are multiplied by the difference between the segment impedance on the source's side and the receiver's side.

$$
P_{\text {path }}=p_{\text {free-field }}\left(Q_{1}-Q_{2}\right) D
$$

TNM also considers attenuation made by tree zones, rows of buildings and atmospheric absorptions; these approaches are from ISO standards. We will not discuss these approaches here in detail.

The total sound pressure for a given vertical geometry is calculated by summing over all the propagation paths for that geometry. The equation is as follows:

$$
P_{\text {rotal }}=\sum_{i=1}^{N} P_{p a t h_{i}}
$$

The final attenuation for a vertical geometry, $A_{s}$, is calculated in reference to the free-field sound pressure as:

$$
A_{s}=20 \times \log _{10}\left|\frac{P_{\text {Total }}}{P_{\text {free-field }}}\right|
$$




\section{CHAPTER V \\ METHODOLOGY}

\subsection{Field Measurements}

In order to evaluate the accuracy of TNM 2.5 and STAMINA 2.0, a series of field measurements with barriers and without barriers were conducted from July 2004 to October 2004. Procedures for field measurements were strictly based on the ANSI Standards (ANSI, 1998) and the Measurement of Highway-Related Noise (Lee, 1996). To determine the effectiveness of the insertion loss of the barriers, two methods for calculating the insertion loss with the barrier were used. One is the indirect BEFORE method at an equivalent site, which requires performing measurements at a site with a barrier to determine AFTER noise levels, and another set of measurements at an "equivalent" site without the presence of the barrier to determine the equivalent BEFORE levels. The BEFORE and AFTER cases for the "indirect" BEOFRE method should be measured simultaneously to insure equivalent conditions of traffic and meteorology. However, it is hard to find an ideal equivalent site. Good engineering judgment should be used on whether or not the adjacent site without barrier is equivalent enough for ground surface or potential influencing factors.

Another method to calculate the insertion loss with a barrier is the "indirect" prediction method, which requires performing measurements at a site with a barrier to determine AFTER noise levels, and predicting the noise level for BEFORE situations by 
using a highway traffic noise-prediction model. Models that comply with certain requirements can be used as the prediction tools. For our research work, the Federal Highway Administration's Traffic Noise Model (FHWA TNM 2.5) and STAMINA 2.0 served this purpose. Since this method depends on the accuracy of the prediction model, it is inherently less accurate compared with the indirect BEFORE method. In both methods, it is required that a reference microphone be set up $1.5 \mathrm{~m}(5 \mathrm{ft})$ at the top of the barrier to account for the traffic equivalence, as shown in Figure 5-1.

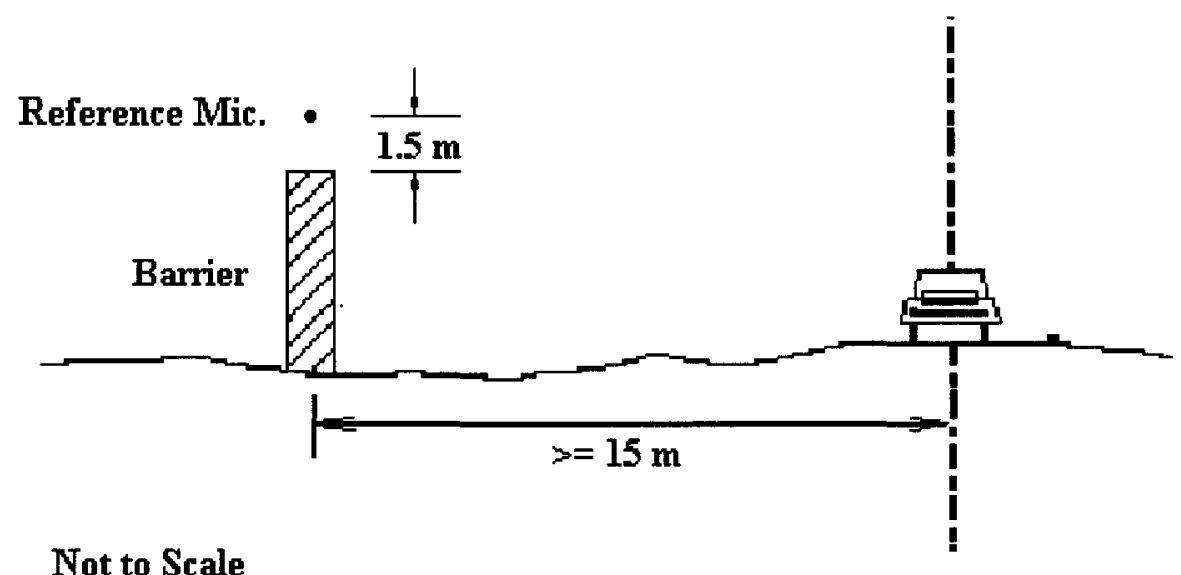

Figure 5-1. Reference microphone at measurement site (Lee, 1996)

In addition to field measurements of insertion loss with barriers, field measurements of ground attenuation on level, flat grassland were also carried out. The procedures complied with the Measurement of Highway-Related Noise (Lee, 1996). Microphones distance up to 122 meters ( $400 \mathrm{ft}$ ) from the centerline of the near lane were measured and compared with predicted noise levels. More detail will be described Chapter VI. 


\subsection{Noise Modeling}

After the field measurements were finished and all the related data for modeling was obtained, noise analysis for specific site by the traffic noise model was carried out. A series of steps can be involved as follows:

1. An assessment of existing conditions and noise levels including field measurement of noise levels, traffic volume, vehicle types, vehicle speeds, roadway layout, area topography etc.

2. Building scenarios for existing conditions by using the FHWA's traffic noise prediction model STAMINA 2.0 and TNM 2.5

3. Prediction of existing and future noise absolute levels and insertion loss

4. Comparison of predicted results with the field measured data for validation. Model accuracy was examined based on the results of noise monitoring and modeling

\subsection{Software Design}

The inconsistencies in TNM 2.5 may be caused by errors in the application of theory and the way by which TNM is handling data. In order to help identify potential errors, new software, HNP 1.0, was designed with Graphic User Interface (GUI), using modifications of the TNM theories contained in the TNM Technical Manual and related sources. Noise levels predicted by HNP 1.0 will be compared to the field measurement data, TNM 2.5, and STAMINA 2.0. Based on the comparisons, parameters related to diffraction and propagation algorithms can be easily adjusted in HNP 1.0 to determine the problem, so as to improve the accuracy of insertion loss in TNM 2.5. 
HNP 1.0 allows the following input types: roadways (including traffic), receivers, barriers, and terrain lines in three dimensions. These input types can be viewed from plan view function. HNP has the function of input file and output file. In addition, HNP 1.0 has the function of zoom in and zoom out for efficient data input. As for the source of noise, it uses the same REMEL data and sub-source height energy splits as TNM 2.5. HNP 1.0 computes the effect of intervening ground by its effective flow with modified ground reflection theory and barrier attenuation with modified diffraction theory compared to TNM 2.5. Atmospheric absorption was also included in HNP 1.0. The interface of HNP 1.0 is shown in Figure 5-2 and Figure 5-3 in Appendix II.

\subsection{Statistical Methods}

With the field measurement data in one barrier site, and two open area sites, comparisons were made to evaluate the model accuracy by comparing the predicted noise levels and measured noise levels. Three models were evaluated:

1. Traffic Noise Model (TNM 2.5)

2. STAMINA 2.0 with national reference energy mean emission level (REMELs)

3. Highway Noise Predictor (HNP 1.0)

These models were selected for the following reasons:

1. the TNM 2.5 is the noise prediction model of latest version, which has been phased in at this time to substitute STAMINA 2.0.

2. STAMINA 2.0 is the noise prediction model which has been used and validated for more than 20 years. Although old, its simplicity for usage and algorithm still excels for engineering applications. 
3. HNP 1.0 is a new noise prediction model with modifications based on the diffraction theory and propagation theory compared to TNM 2.5. It can make a direct comparison with TNM 2.5 for the problem determination and for the later improvements in TNM 2.5 .

To compare these three models, statistical analysis is used to compare the measured noise levels with modeled results. Several statistical tests are used, with methods that are included the followings:

- Minimum and maximum error

- Standard deviation

- Mean

- Range

\subsubsection{Paired two-tailed t-test}

In order to statistically compare the predicted results of TNM 2.5 and HNP 1.0 with the measured results, a paired two-tailed t-test method is used. This method is chosen for the comparison of highway noise levels for obvious reasons as following: the data represents a small sample size. The calculated and measured sound levels from each combination of TNM 2.5 or HNP 1.0 and measured data represent samples from a single point source; and the sample can be assumed to be normally distributed (Cohn, 2001).

For each receiver distance, the sample consists of the mean difference between the measured and predicted values. The mean difference is then used to calculate the standard deviation of the sample, which is in turn used to calculate a t-value. The calculated $t$ value is then compared with a tabulated critical $t$-value for the desired level 
of significance. If the calculate $t$-value is grater than the critical t-value, then it can be stated that the difference between predicted and measured value is caused by some factors other than chance. This indicates that the difference between the predicted and measured values is significant. On the other hand, if the calculated t-value is less than the critical t-value, it means that the difference between predicted and measured data can be attributed to chance and the difference is not significant.

In this research, a level of significance of $5 \%$ will be used, which indicates that the probability of error at any given distance is $5 \%$ and $95 \%$ confidence level in the results of the predicted data can be achieved. 


\section{CHAPTER VI}

\section{FIELD MEASUREMENTS OF HIGHWAY BARRIER INSERTION LOSS}

\subsection{Measurement Sites}

Field measurement of highway noise is a direct and effective way for model validation. Four sites were selected for both measurements and modeling, including two barrier sites along Interstate 264 in Louisville, $\mathrm{KY}$, and two open area sites, one along Interstate 264, the other near roadway 111 in New Albany, IN. Specific site parameters (geometry of site, traffic volume, traffic classification, traffic speed, wind speed, wind direction, temperature, relative humidity) were carefully recorded during measurements and modeling with FHWA TNM 2.5 and STAMINA 2.0. The predicted noise levels were then compared with the field measured data. Based on the comparison, propagation theories and barrier effectiveness can be evaluated and validated. To obtain the most accurate and high quality acoustical data, sites should be selected based on certain requirements.

\subsection{Site Requirements}

Measurement sites should have the common characteristics that can be modeled by TNM 2.5 and it should be relatively simplistic to isolate individual features of TNM (Rochat, 2004). The selection of our sites included two barrier sites and two open area sites as follows: 


\subsubsection{Site 01KY}

The measurement site was located near Exit 18 of Interstate 264. Measurements were taken on several days from May 2004 to August 2004. The highway noise barrier was built to protect resident houses on its Northern Side. It had a height of $3.9 \mathrm{~m}(12.7 \mathrm{ft})$ at the measurement location. The site was influenced by heavy traffic volume and experienced high noise levels. At the receiver height of $1.5 \mathrm{~m}(5 \mathrm{ft})$, the line of sight for heavy truck stacks was blocked by the barrier. The main ground type at the receiver side was lawn, up to a $30 \mathrm{~m}(100 \mathrm{ft}$ ) distance from the barrier. Receivers were set up $15 \mathrm{~m}$ (50 $\mathrm{ft}), 30 \mathrm{~m}(100 \mathrm{ft}), 61 \mathrm{~m}(200 \mathrm{ft})$, and $122 \mathrm{~m}(400 \mathrm{ft})$ away from the barrier. A reference microphone was placed $1.5 \mathrm{~m}(5 \mathrm{ft})$ above the barrier, as shown in Figure 6-1 in Appendix III. Because the first row of houses was $37 \mathrm{~m} \mathrm{(120} \mathrm{ft)} \mathrm{away} \mathrm{from} \mathrm{the} \mathrm{barrier,}$ possible problems in modeling may have included the close spacing of resident houses,

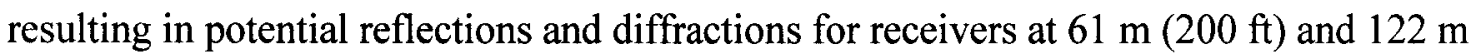
$(400 \mathrm{ft})$. Therefore, receivers at a distance of $15 \mathrm{~m}(50 \mathrm{ft})$, and $30 \mathrm{~m}(100 \mathrm{ft})$ were selected as ideal receiver positions for noise measurements. Receiver distances of $61 \mathrm{~m}(200 \mathrm{ft})$

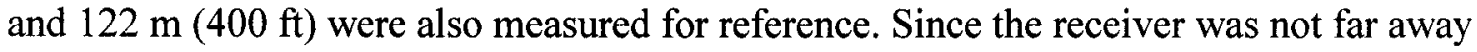
from the barrier and there were no other unknown noise sources located near the barrier sites, such as airports, construction sites, etc, therefore, the background noise did not contaminate the noise levels at the receivers.

\subsection{2 $\underline{\text { Site 02KY }}$}

The measurement site was located near Exit 17 of Interstate 264. Measurements were taken on the day of August 27, 2004. The highway noise barrier was built to protect 
resident houses on its Southern Side. It had a height of $5.2 \mathrm{~m} \mathrm{(17} \mathrm{ft)} \mathrm{at} \mathrm{the} \mathrm{measurement}$ location. The site was influenced by heavy traffic volume and experienced a high noise level. At the receiver height of $1.5 \mathrm{~m}(5 \mathrm{ft})$, the line of sight for heavy truck stacks was blocked by the barrier. The main ground type at the receiver side was lawn, up to a $30 \mathrm{~m}$ (100 ft) distance from the barrier. Receivers were set up $15 \mathrm{~m}(50 \mathrm{ft}), 30 \mathrm{~m}(100 \mathrm{ft})$ away

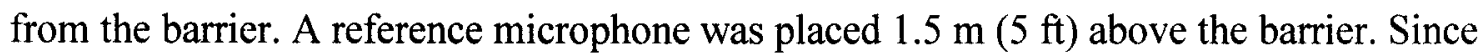
the receiver was not far away from the barrier and there were no other unknown noise sources located near the barrier sites, such as airports, construction sites, etc, therefore, the background did not contaminate the noise levels at the receivers.

\subsubsection{Site 03KY}

According to the ANSI 1998, an equivalent site $03 \mathrm{KY}$ for barrier site $01 \mathrm{KY}$ was also selected for the measurement of BEFORE noise levels. This site was a recreation park with large open grassland. It was on the same side of barrier site 01KY. It had similar ground conditions and similar meteorological conditions and the same traffic conditions as those of site $01 \mathrm{KY}$. This site enabled the calculation of indirect method for insertion loss.

\subsubsection{Site 04IN}

Measurements were taken along roadway 111 in New Albany, $\mathbb{I N}$ on three days: October 1,3 , and 7,2004 . The roadway was a two-lane minor roadway with free flow traffic volume; the dominant traffic speed was above $50 \mathrm{mph}$. The traffic volume on this roadway was light. On the South Side of this roadway was a large, even grassland space 
for our measurements, as shown in Figure 6-2 in Appendix III. On the North Side of the roadway was a large cornfield. This site was free of large reflecting surfaces, such as

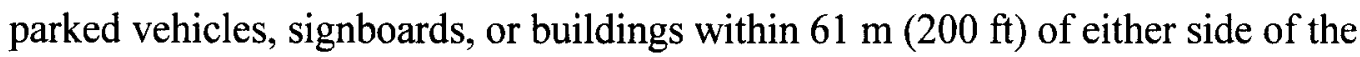
highway traffic path or the microphones. The background noise level at this site was very low, composed of sounds from birds and tree leaves rustling. The site was not located near known noise sources.

These site plans were obtained from related local agencies and digitized with GIS software; these site plans were of sufficient detail to facilitate accurate modeling in TNM 2.5. In addition, photos were taken to assist in the selection process.

The ground type of the open area and the barrier site at the receiver site was acoustically soft. In TNM 2.5 modeling, lawn ( 300 cgs Rayls) was selected as the ground type. The barrier sites were mostly flat, although there was a ditch of about $1.2 \mathrm{~m}(4 \mathrm{ft})$ deep between the shoulder of the pavement and the barrier.

\subsection{Instrumentation}

This section describes field measurement instrumentations, including acoustic measurement devices, a traffic volume recorder, and a meteorological device.

\subsubsection{Microphone System}

A microphone transforms sound pressure variations into electrical signals, and is measured by a sound level meter. A compatible preamplifier should also be used for high-input impedance and constant, low-noise amplification over a wide frequency range. The microphone system needs to be supported by a tripod, but the position of the 
microphone should be placed behind the tripod to minimize the reflection from the support device (Cohn, 1998). The Larson Davis 824 and 812 microphone systems with preamplifiers were used in the current research. Foam windscreens were placed atop each microphone to reduce the effects of wind-generated noise. A cable of $15 \mathrm{~m}(50 \mathrm{ft})$ was used for reference microphone $1.5 \mathrm{~m}(5 \mathrm{ft})$ above the top of the noise barrier. Typically, measurement frequencies from $50 \mathrm{~Hz}$ to $10 \mathrm{kHz}$ in the $1 / 3$-octave band will satisfy the objectives of highway noise study. The sound level meter was set up to continuously measure the overall A-weighted equivalent sound levels in a 15 minute period.

\subsubsection{Digital Audio Tape (DAT) Recorder}

The DAT recorder is an ancillary device for highway noise recording. Samples of noise levels on the reference receiver and on receivers at varying distance from the barrier were recorded during each measurement. The data can be transferred to computers in the lab for later frequency analysis.

\subsubsection{Traffic Analysis Instrumentation}

The instrumentation for real time traffic recording included a video camera and the speed gun. The video camera was deployed to continuously record traffic volume. Later the data were processed to determine vehicle counts and categorization by manual analysis in the lab. The video camera was placed on the side of the highway at a higher point, recording the traffic from an angle. Each videotape had a length of two hours for recording time. The speed gun was used for dominant traffic speed measurements. The 
speed gun was shot at different types of vehicles several times in a run. The speed for each type can be obtained by averaging the traffic speeds.

\subsubsection{Meteorological Instrumentation}

In addition to acoustical and traffic instrumentation, a meteorological instrumentation was also deployed. The device was set up at certain height above the ground to measure temperature, relative humidity, wind speed and direction. The data were recorded in sufficient detail to aid in possible future incorporation by TNM 2.5.

\subsubsection{GIS Software}

Software for the Geography Information System (ArcView) was used to extract the coordinates of all important site features, including microphone positions, roadways, barriers, and undulations of ground surface. With the help of GIS software, it is easy to build the model for TNM 2.5 noise prediction.

\subsection{Field Measurement Procedures}

Since wide variation in site geometries influenced the measurement system setup, placement of the measurement instrumentation, including microphones, meteorological systems and traffic analysis systems, depended greatly on the presence of a noise barrier, terrain features, and accessibility. All of the data were collected and analyzed in general conformance the ANSI standards (1998) and the FHWA's procedures (Lee, 1996). 


\subsubsection{Measurement System Setup}

For the acoustical measurements, the microphones were placed in a line perpendicular to the roadway at up to three distances from the centerline of the near lane for one open area site or at up to four distances from the centerline of the noise barriers for barrier sites. For the open area site, the reference receiver was place at $15 \mathrm{~m}(50 \mathrm{ft})$ from the centerline of the near lane. Another microphone was placed at $30 \mathrm{~m}(100 \mathrm{ft})$ and $61 \mathrm{~m}(200 \mathrm{ft})$ consecutively, because of the site permitting. All microphone heights were

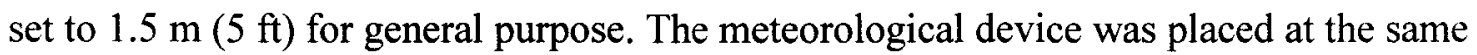
height as the receiver. For the barrier site, since the distance from the barrier to the near lane of roadway was greater than $15 \mathrm{~m}(50 \mathrm{ft})$, the reference microphone was placed 1.5 $\mathrm{m}(5 \mathrm{ft})$ above the noise barrier. The farthest distance for the receiver is at $122 \mathrm{~m}(400 \mathrm{ft})$ away from the noise barrier. The meteorological device was placed at the same height. All microphones except for the reference microphone were set up $1.5 \mathrm{~m}(5 \mathrm{ft})$ above the ground. The meteorological device was placed at the height of $1.5 \mathrm{~m}(5 \mathrm{ft})$.

Following is a step-by-step description of the measurement system setup for our measurement at each measurement site:

(1). For each site, microphone positions were predetermined based on the highway plan and adjusted in the field. Markers were used to mark the position of each receiver point. If possible, a short length of rebar was driven into the ground at each position to secure tripods and masts.

(2). The microphone system with preamplifiers and windscreens, was set up and attached the microphone system to a tripod at specified height directly above the local ground 
surface. The microphone system with sound level meters was connected and put aside the sound level meter in sufficient distance to minimize influence to the microphone.

(3). The meteorological device was positioned in a place that can be representative of the meteorological conditions at the nearby microphones. Each meteorological device was placed at a sufficient distance from the microphone location so as to allow personnel to make periodic checks of system functionality without influencing the acoustical measurements.

(4). To avoid acoustical contamination of acoustical instrumentation, microphone cable was sufficient long and all the clock of all related measurement instrumentation was synchronized.

(5). A preliminary sound level calibration of the system was performed to ensure that each equipment functioned properly. The error of calibration for each equipment was within requirements.

(6). The windscreen was then deployed and the preamplifier cable was secured to ensure stability of the equipment.

(7). The video camera was positioned on the side of the highway at the highest point possible in order to have a clear image of all traffic volume, if there is no overpass above the highway. The cable of video camera was connected to the power device that connected to the car cigarette lighter or mobile power device.

(8). Continuous meteorological data collection was then initiated.

(9). Traffic noise level measurements with the sound level meter, and DAT were initiated. (10). Continuous video recordings were initiated. 
(11). After measurements, a post-measurement sound level meter calibration was performed and changes in calibration data were documented.

(12). All instrumentation was powered down and the entire system disconnected and stored.

\subsection{Data Reduction and Data Analysis}

Since TNM 2.5 currently calculated sound levels for a windless environment, the measurement data beyond this requirement was reduced for quality insurance of data, according to the wind qualifications in current ANSI specifications (ANSI 1998), "calm" for speeds never exceeding $1 \mathrm{~m} / \mathrm{s}$.

As for input to TNM 2.5, the video traffic data were analyzed in 15 -minute blocks using manual counts in the lab. Data extracted from the videotapes were supplied to vehicle categories and traffic volume for each category. Since the volume of motorcycles and buses was comparatively small, three vehicle categories were applied: automobiles, medium trucks, and heavy trucks. The 15-minute totals were scaled to vehicles per hour for TNM 2.5 input. Average speeds were calculated with the data measured by the speed gun in the field, and then applied to all present vehicle categories for that data block.

\subsubsection{Results and Analysis}

\subsubsection{Measurement of Absolute Sound Levels}

The absolute sound levels are defined as the actual sound levels measured at a

microphone location. Table 6-1 contains the average values for all 15 minute $L_{A e q}$ sound levels measured at each of the standard microphone positions for the two barrier sites and 
two open sites. The Reference levels ranged from 82.5 to $82.7 \mathrm{dBA}$, the noise levels behind the barrier ranged from 58.0 to $67.9 \mathrm{dBA}$ for the site $01 \mathrm{KY}$ and ranged from 64.8 to $65.2 \mathrm{dBA}$ at the site $02 \mathrm{KY}$.

\subsubsection{Computer Modeling of Absolute Sound Levels}

Field measurement data were compared with computer models TNM 2.5 and STAMINA 2.0 to test the accuracy of the prediction model. Table 6-1 includes the average error summaries of this testing for TNM 2.5 and STAMINA 2.0 (using the older National REMELs)

Table 6-1.

Measured and Predicted noise levels (dBA) at site $01 \mathrm{KY}, 02 \mathrm{KY}, 03 \mathrm{KY}$, and 04IN

\begin{tabular}{lcccccc}
\hline Site ID & $\begin{array}{c}\text { Mic } \\
\text { Location }\end{array}$ & $\begin{array}{c}\text { Measured } \\
\text { levels }\end{array}$ & $\begin{array}{c}\text { Predicted } \\
\text { TNM 2.5 }\end{array}$ & $\begin{array}{c}\text { Predicted } \\
\text { STAMINA2.0 }\end{array}$ & $\begin{array}{c}\text { TNM - } \\
\text { Measured }\end{array}$ & $\begin{array}{c}\text { STAMINA - } \\
\text { Measured }\end{array}$ \\
\hline $01 \mathrm{KY}$ & Ref & 82.7 & 78.6 & 80.1 & -4.1 & -2.6 \\
(barrier site) & $50 \mathrm{ft}$ & 67.9 & 64.6 & 68.4 & -3.3 & 0.5 \\
& $100 \mathrm{ft}$ & 66.8 & 64.0 & 68.5 & -2.8 & 1.7 \\
& $200 \mathrm{ft}$ & 63.0 & 63.3 & 67.1 & 0.3 & 4.1 \\
& $400 \mathrm{ft}$ & 58.0 & 61.8 & 64.3 & 3.8 & 6.3 \\
$02 \mathrm{KY}$ & $\mathrm{Ref}$ & 82.5 & 78.0 & 79.8 & -4.5 & -2.7 \\
(barrier site) & $50 \mathrm{ft}$ & 65.2 & 62.5 & 65.6 & -2.7 & 0.4 \\
& $100 \mathrm{ft}$ & 64.8 & 61.7 & 65.2 & -3.1 & 0.4 \\
$03 \mathrm{KY}$ & $50 \mathrm{ft}$ & 82.1 & 79.7 & 80.9 & -2.4 & -1.2 \\
(open area) & $100 \mathrm{ft}$ & 76.7 & 77.0 & 75.5 & 0.3 & -1.2 \\
& $200 \mathrm{ft}$ & 71.8 & 74.0 & 72.0 & 2.2 & 0.2 \\
& $400 \mathrm{ft}$ & 67.0 & 69.5 & 68.0 & 2.5 & 1.0 \\
$04 \mathrm{IN}$ & $50 \mathrm{ft}$ & 65.8 & 67.0 & 67.0 & 1.2 & 1.2 \\
(open area) & $100 \mathrm{ft}$ & 60.4 & 62.3 & 62.7 & 1.9 & 2.3 \\
& $200 \mathrm{ft}$ & 53.4 & 56.6 & 58.6 & 3.2 & 5.2 \\
\hline
\end{tabular}


Table 6-2. Statistical analysis of errors at sites $01 \mathrm{KY}, 02 \mathrm{KY}, 03 \mathrm{KY}$, and $04 \mathrm{IN}$

\begin{tabular}{lcc}
\hline Variables & TNM 2.5 dBA: $L_{A e q}$ & STAMINA 2.0 dBA: $L_{A e q}$ \\
\hline Min & -4.50 & -2.70 \\
Max & 3.80 & 6.30 \\
Mean & -0.50 & 1.04 \\
variance & 8.26 & 6.82 \\
\hline
\end{tabular}

Table 6-2 shows that TNM performs the best in two out of the four statistical categories evaluated. STAMINA 2.0 outperformed TNM 2.5 with an average minimum error of $-2.70 \mathrm{dBA}$, and with variance of $6.8 \mathrm{dBA}$. TNM 2.5 out-performed STAMINA 2.0 with an average maximum error of $3.8 \mathrm{dBA}$ and mean of $-0.5 \mathrm{dBA}$. Large variance in both models shows significant scatter in the data, this means that the accuracy in both models still needs to be improved.

\subsubsection{Insertion Loss Calculation}

Table 6-3.

Predicted insertion loss (dBA) by TNM 2.5 and STAMINA 2.0 at the site $01 \mathrm{KY}, 02 \mathrm{KY}$

\begin{tabular}{lllll}
\hline Site & Mic. & Predicted & Predicted & TNM 2.5 - \\
& Location & TNM 2.5 & STAMINA2.0 & STAMINA 2.0 \\
\hline $01 \mathrm{KY}$ & $50 \mathrm{ft}$ & 11.7 & 6.4 & 5.3 \\
(barrier site) & $100 \mathrm{ft}$ & 10.7 & 4.4 & 6.3 \\
& $200 \mathrm{ft}$ & 8.5 & 3 & 5.5 \\
& $400 \mathrm{ft}$ & 6.2 & 2.1 & 4.1 \\
$02 \mathrm{KY}$ & $50 \mathrm{ft}$ & 12.6 & 8.8 & 3.8 \\
(barrier site) & $100 \mathrm{ft}$ & 9.7 & 7.1 & 2.6 \\
\hline
\end{tabular}

Table 6-3 shows that the TNM 2.5 over-predicts insertion loss ranging from 2.6 dBA to 6.3 dBA compared with STAMINA 2.0, averaging $4.6 \mathrm{dBA}$ for these two barrier sites. Insertion loss depends on many factors, not only on barrier attenuation, but also on 
ground effects, site geometry, even on the selection of receivers. However, from the measurements and compared with predicted noise levels, it has been proved that TNM 2.5 still over-predicts the insertion loss by several dBA.

For the barrier site $01 \mathrm{KY}$, an equivalent site $03 \mathrm{KY}$ was available to make a more direct comparison of insertion loss. This equivalent site was on the same side of the barrier site and it had no barrier. The measured insertion loss at $15 \mathrm{~m}(50 \mathrm{ft})$ behind barrier was average $6.1 \mathrm{dBA}$ and less on further distances from the barrier. It was concluded that the insertion loss predicted by STAMINA 2.0 in this case was in very good agreement with the measured insertion loss. On the contrary, TNM 2.5 gave too much credit on the effectiveness of the barrier, which led to unrealistic over-prediction of the insertion loss.

Since the measurement data for AFTER barrier situation were collected, the indirect ANSI method was used to determine the insertion loss. The insertion loss was calibrated to the reference microphone. This calibration value was the difference between model prediction sound levels and measured sound levels at the reference microphone. Table 6-4 shows the adjusted insertion loss for TNM 2.5, STAMINA 2.0, and the insertion loss difference of the two models.

\subsubsection{Adjusted Insertion Loss Calculation}

Table 6-4 shows that TNM 2.5 over-predicts insertion loss compared with STAMINA 2.0 ranging from $0.8 \mathrm{dBA}$ to $3.4 \mathrm{dBA}$, averaging $2.6 \mathrm{dBA}$ for these two barrier sites after calibration. However, the adjusted insertion loss in both TNM 2.5 and STAMINA 2.0 deviate from the measured insertion loss further and further for all the 
receivers, ranging from $10.9 \mathrm{dBA}$ to $13.9 \mathrm{dBA}$ for TNM 2.5 and 8.3 to $10.7 \mathrm{dBA}$ for

STAMINA 2.0. Based on engineering judgment, it is hard to achieve a $12 \mathrm{dBA}$ insertion loss for a $3.9 \mathrm{~m}(12.7 \mathrm{ft})$ height noise barrier under such conditions. In addition, we can see that after calibration, the insertion loss at further distance from the barrier is larger than the insertion loss at near distance in both STAMINA 2.0 and TNM 2.5.

Table 6-4.

Adjusted Insertion loss (dBA) by TNM 2.5 and STAMINA 2.0 at the site $01 \mathrm{KY}, 02 \mathrm{KY}$

\begin{tabular}{lllll}
\hline Site & $\begin{array}{l}\text { Mic. } \\
\text { Location }\end{array}$ & $\begin{array}{l}\text { Predicted } \\
\text { TNM 2.5 }\end{array}$ & $\begin{array}{l}\text { Predicted } \\
\text { STAMINA2.0 }\end{array}$ & $\begin{array}{l}\text { TNM 2.5 - } \\
\text { STAMINA 2.0 }\end{array}$ \\
\hline $01 \mathrm{KY}$ & $50 \mathrm{ft}$ & 12.2 & 9.2 & 3.0 \\
(barrier site) & $100 \mathrm{ft}$ & 11.7 & 8.3 & 3.4 \\
& $200 \mathrm{ft}$ & 12.4 & 9.2 & 3.2 \\
& $400 \mathrm{ft}$ & 13.7 & 10.7 & 3.0 \\
$02 \mathrm{KY}$ & $50 \mathrm{ft}$ & 13.9 & 11.5 & 2.4 \\
(barrier site) & $100 \mathrm{ft}$ & 10.9 & 10.1 & 0.8 \\
\hline
\end{tabular}

These inconsistencies may be due to the following reasons: first, the ANSI indirect method mainly based on the reference receiver for calibration. If there exists a large difference between the predicted sound level at the reference receiver and the measured sound level at the reference receiver, this error may contribute to the total error for the insertion loss. Second, the ANSI indirect method is a linear method for calibration, but the sound interaction behind the barrier is a complex phenomenon, especially considering receivers at varying distances from the barrier. By using linear method with a nonlinear system will result in excessive errors. Therefore, in order to obtain more accurate predicted insertion loss, more concerns should be put on the accuracy of the model itself. 


\section{CHAPTER VII}

\section{MODEL VERIFICATION}

\subsection{Verification of TNM Validation Cases with a Point Source}

The first part provides the comparisons and verifications of the HNP 1.0 to the results of TNM 2.5 and the measurements. Comparisons are made to five different data sets: three involved point source geometries and the remaining two involved comparisons of simple single line source and multiple line sources. The first verification is to compare with Embleton's model for the reflection from the ground of finite impedance (Embleton, 1983). The second verification is to compare ground effect with the measurements by Parkin and Scholes over grassland (Parkin, 1965). The third verification is to compare the insertion loss of a noise barrier by Scholes, also over grassland (Scholes, 1971). Since TNM validations are mainly based on the above three verification cases of point source, HNP will also provide detailed comparisons for all these cases. Overall, the agreement between the results of HNP and the measurements is found to be very satisfactory and out-performs that of TNM 2.5.

The second part provides the comparison of the diffraction theory in TNM 2.5, STAMINA 2.0 and HNP 1.0.

The third part provides the comparisons of insertion loss for the four propagation path model by Janasson (De Jong, 1983) and the two propagation path model. HNP 1.0 
applies the four propagation paths to calculate noise levels behind the barrier and TNM 2.5 uses the two propagation path model.

\subsubsection{Ground Reflection Model}

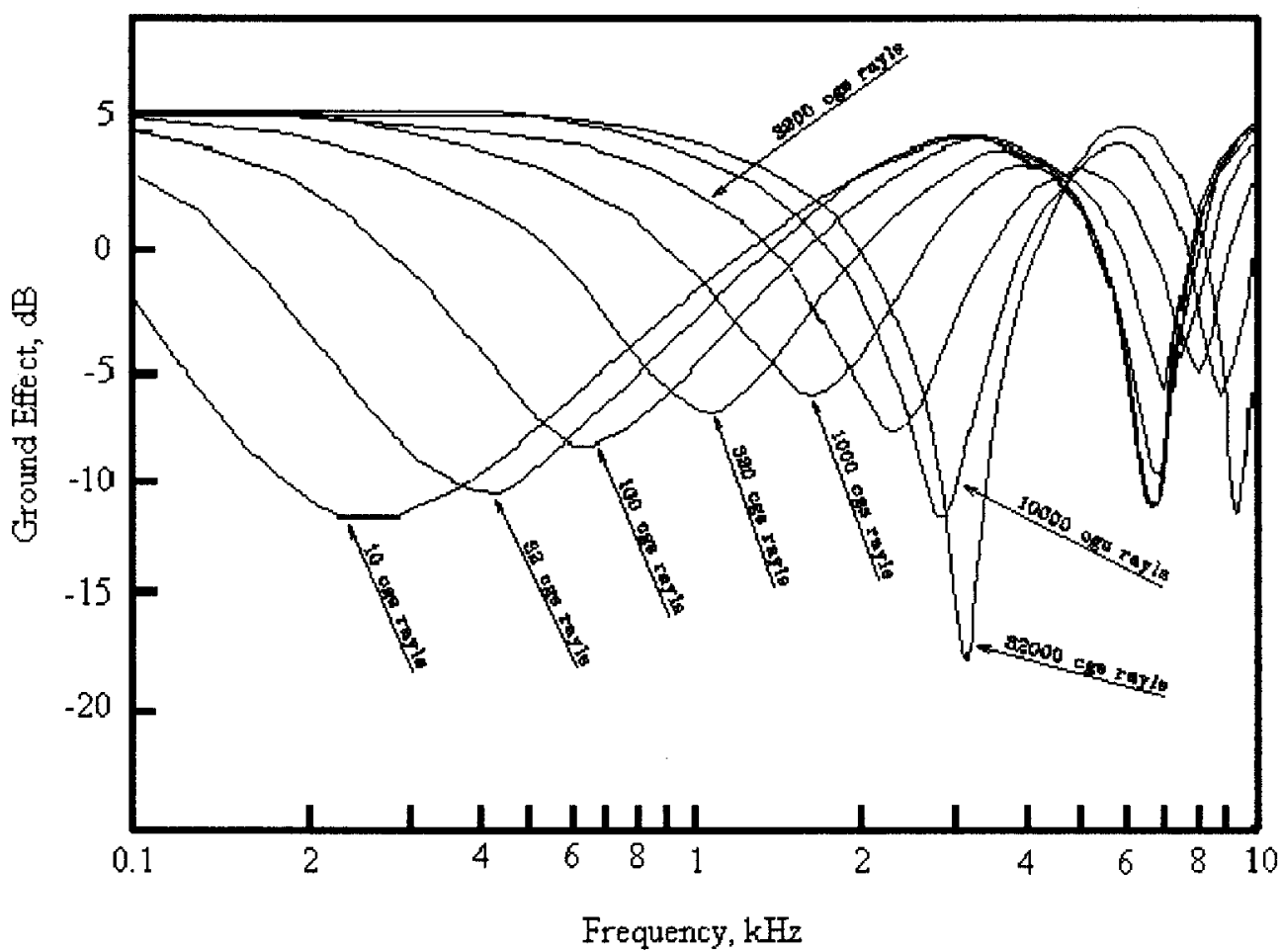

Figure 7-1. Set of predicted spectra for various values of flow resistivity of the ground surface. Reference sound pressure level is that which would exist at the receiver in free field (Embleton, 1983)

TNM deploys the ground reflection model which is based on the approach of

Chessell (Chessell, 1977). This model incorporates the single-parameter ground impedance model first proposed by Delany and Bazley (Delany, 1970). Later, Embleton, Piercy and Daigle conducted the measurements to determine the empirical relationship between ground types and effective flow resistivity (EFR) (Embleton, 1983). Figure 7-1 presents theoretical plots of Embleton's model for the following published geometry configurations and values of EFR: Source height $=0.31$ meters $(1.0$ foot $)$; receiver height 
$=1.22$ meters $(4.0$ feet $)$; source-to-receiver distance $=15.2$ meters $(50$ feet $)$. The values of EFR are from very soft ground type (powder snow, EFR $=10 \mathrm{cgs}$ Rayls) to hard ground type (10,000 cgs Rayls).

In Figure 7-1, y-axis denotes excess attenuation by the ground effect in dBA, which stands for the difference between the free-field (no-ground) condition and the condition with the ground for the given frequency band. At low frequency bands, there is a $6 \mathrm{dBA}$ addition to the ground effects due to doubling of sound pressure. In the middle frequency bands, there exists a "ground-effect dip", or "frequency dip", due to the complex interaction between sound reflection on the ground and the direct sound wave.

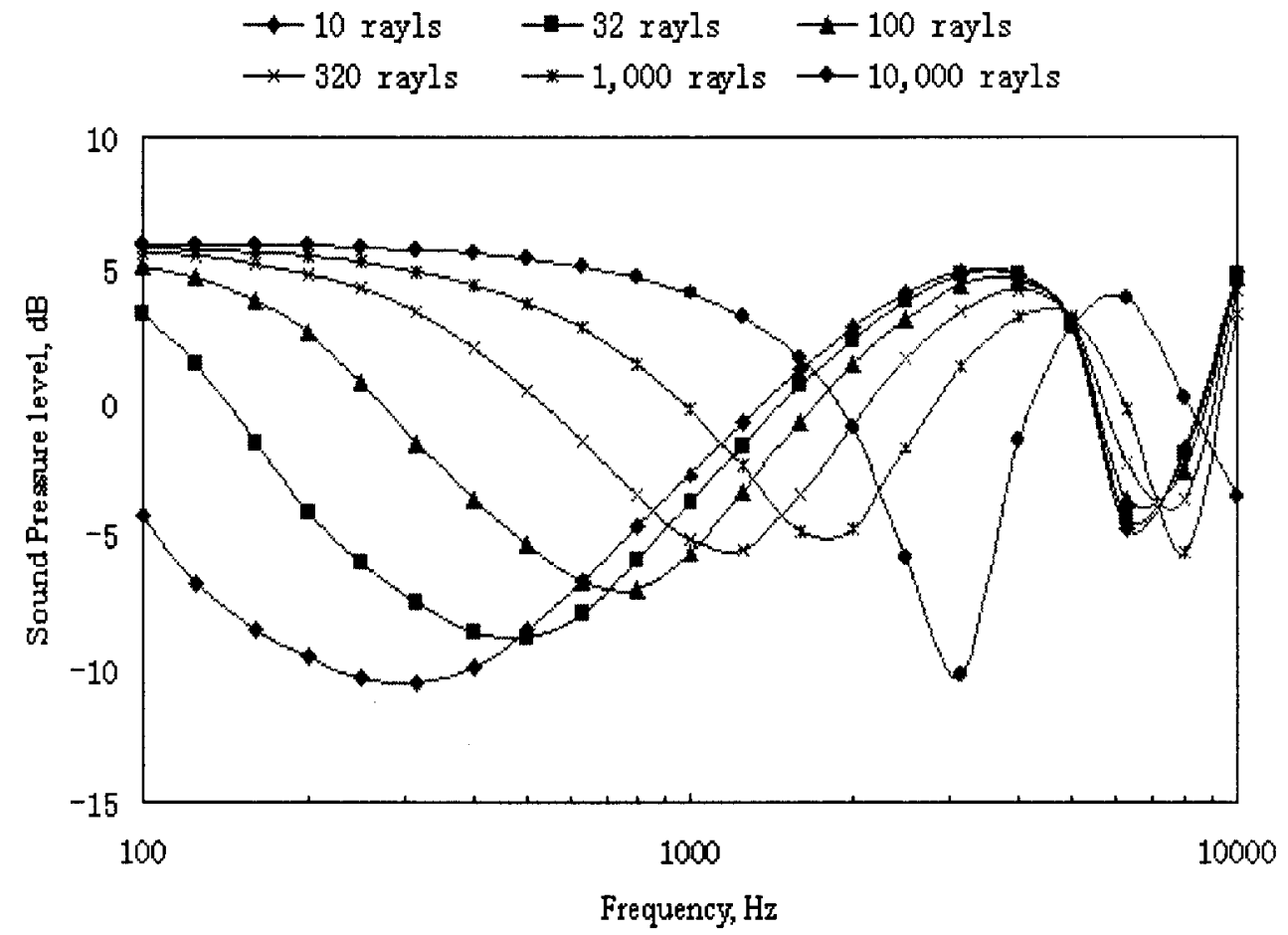

Figure 7-2. Set of HNP predicted spectra for the same values of flow resistivity of the ground surface of Embleton's model

The HNP is also based on the approach of Chessell (1977); however, it utilizes a different method to calculate the ground wave function, as described in Chapter IV. The plot for the comparison of the ground effect for HNP by using same geometry 
configurations and EFR values of Embleton is shown in Figure 7-2. The prediction of the ground effect by HNP is in agreement with the Embleton's plot.

\subsubsection{Measurements over Grassland}

In this section, the reflection model of HNP 1.0 is compared with that of TNM 2.5 and measurements of sound propagation over grassland by Parkin and Scholes (Parkin, 1965), which was conducted on the site at Hatfield. Hatfield was an airport with large grassland up to $0.05 \mathrm{~m}$ ( 2 inches) high. The ground was especially flat, within \pm 0.3 meters ( \pm 1 foot) for more than 1098 meters ( 3600 feet). A series of measurements were made with different geometry configurations and under varying meteorological conditions. Since TNM 2.5 does not take into account the meteorological conditions, comparisons are performed based on normal atmospheric conditions, that is, normal temperature gradient with no strong lapse or inversion and zero vector wind with no components in the source-to-receiver direction. The source was a jet engine at a height of 1.8 meters ( 6 feet) and the microphone was located at a height of 1.5 meters ( 5.0 feet) above the ground surface. The following distances are selected for the comparisons in

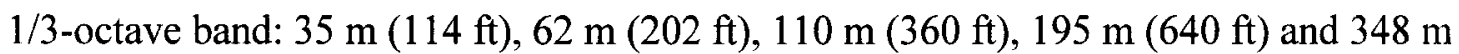
(1140 ft). An EFR of 325 cgs Rayls is selected as most likely to represent the characteristics of the ground type at Hatfield. Figure 7-3 through Figure 7-7 presents the comparisons of measured and modeled ground effect by TNM 2.5 and HNP 1.0 in 1/3octave bands.

The main features of these curves can be summarized as follows: (1) At low frequencies, the excess attenuation curves show positive excess attenuation values due to the in-phase addition of the direct and reflected waves. (2) These curves exhibit a 
minimum (a maximum excess attenuation) at approximately $500 \mathrm{~Hz}$ due to the phase shift of the reflected wave. The depth of minimum increases with increased sourcereceiver separation.

Overall, TNM 2.5 is in good agreement with the measurements in low frequency bands and high frequency bands. However, in the middle frequency bands around $500 \mathrm{~Hz}$, where the maximum excess attenuation occurs as a result of direct sound waves interacting with the reflected waves, together with an absorptive ground surface, TNM 2.5 predicts less satisfactory results compared with the measurements. In most cases, the errors are more than $5 \mathrm{dBA}$ at the ground effect dip. However, HNP 1.0 outperforms TNM 2.5 compared with the measurements, especially at the ground effect dip.

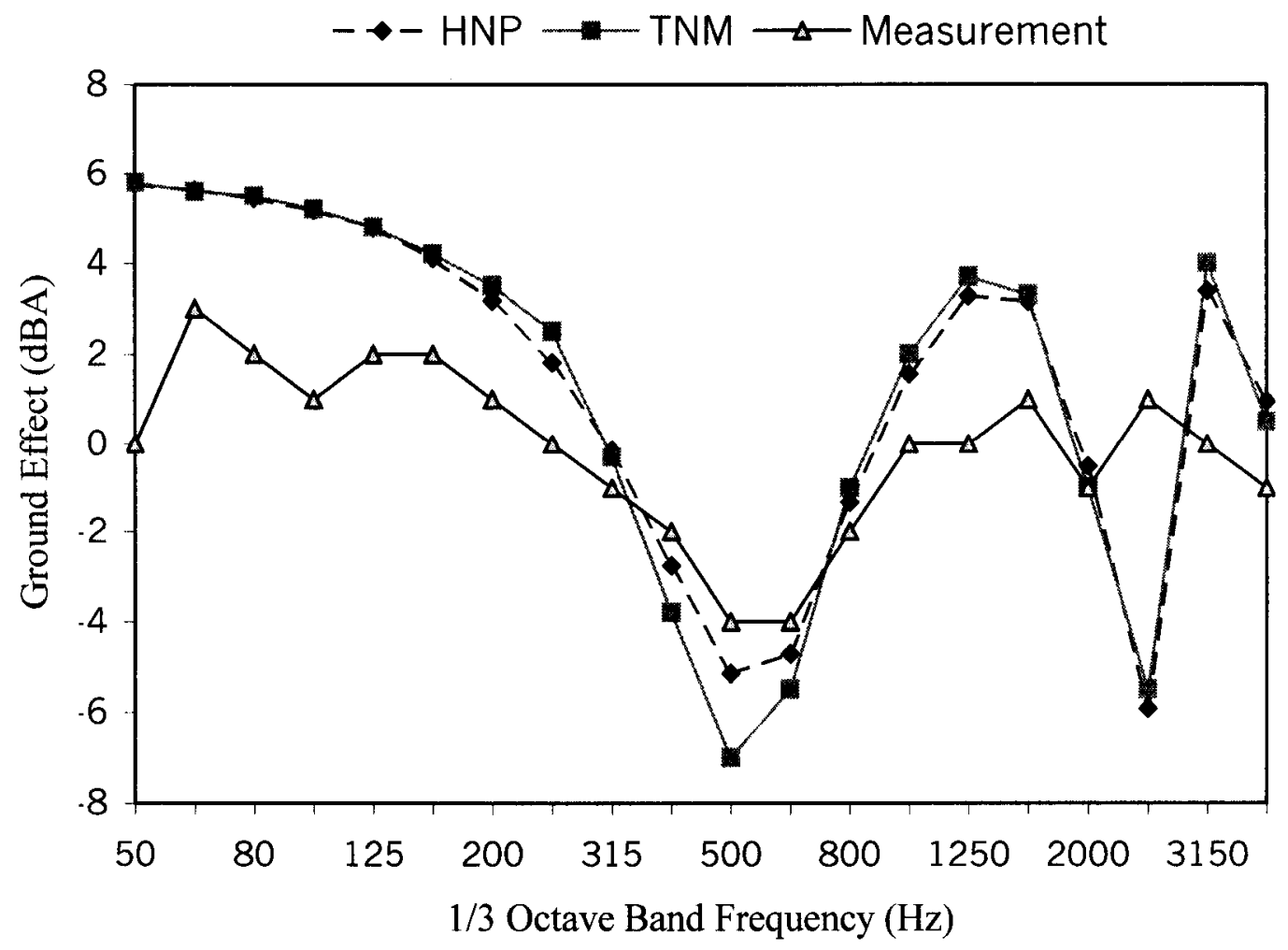

Figure 7-3. Comparison with the measurement by TNM and HNP over grassland, source receiver distance $35 \mathrm{~m}(114 \mathrm{ft})$ 
As can be seen in Figure 7-3, TNM 2.5 and HNP 1.0 demonstrate almost the same ground effect in the low frequency bands up to $315 \mathrm{~Hz}$ and high frequency bands from $1000 \mathrm{~Hz}$. In the middle frequency bands, both models predict the exact frequency position for the first ground effect dip, which occurs at $500 \mathrm{~Hz}$. However, the error at this ground effect dip for TNM 2.5 is about $3 \mathrm{dBA}$ compared to the measurement data, based on the assumption that the measurements of the ground effect are correct. The error between HNP 1.0 and the measurement data at ground effect dip is about $1 \mathrm{dBA}$.

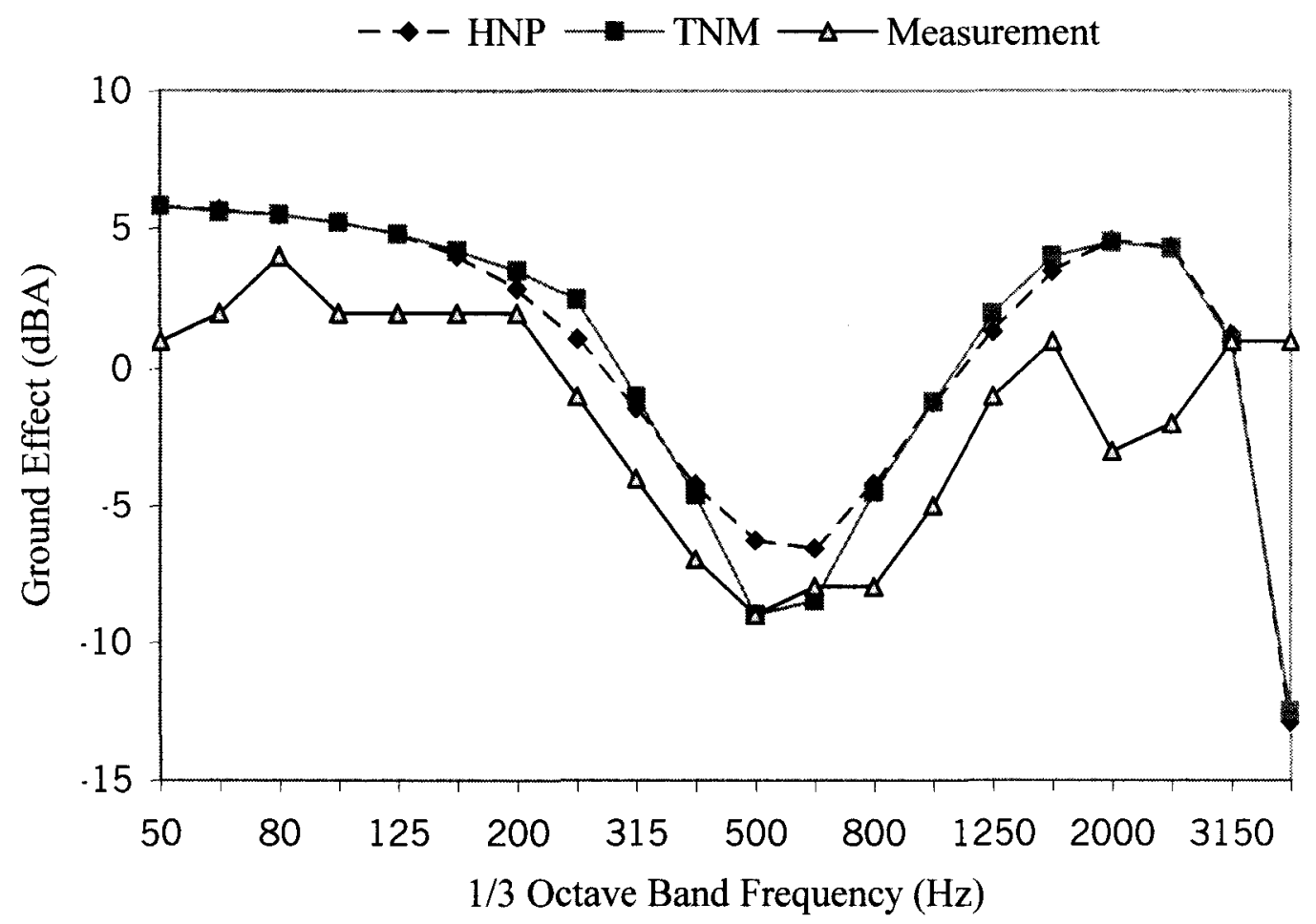

Figure 7-4. Comparison with the measurement by TNM and HNP over grassland, source receiver distance $62 \mathrm{~m}(202 \mathrm{ft})$

As can be seen in Figure 7-4, TNM 2.5 and HNP 1.0 demonstrate almost the same ground effect in the low frequency bands up to $400 \mathrm{~Hz}$ and high frequency bands from $800 \mathrm{~Hz}$. In the frequency of $250 \mathrm{~Hz}, \mathrm{HNP} 1.0$ out-performs TNM 2.5 by $1.5 \mathrm{dBA}$ compared with the measured data. In the middle frequency bands, both models predict 
the exact frequency for the first ground effect dip, which occurs at $500 \mathrm{~Hz}$. The error at this ground effect dip for HNP is $2.7 \mathrm{dBA}$ compared to the measurement data, based on the assumption that the measurements of the ground effect are correct. TNM is in good agreement with the measurements in this case.

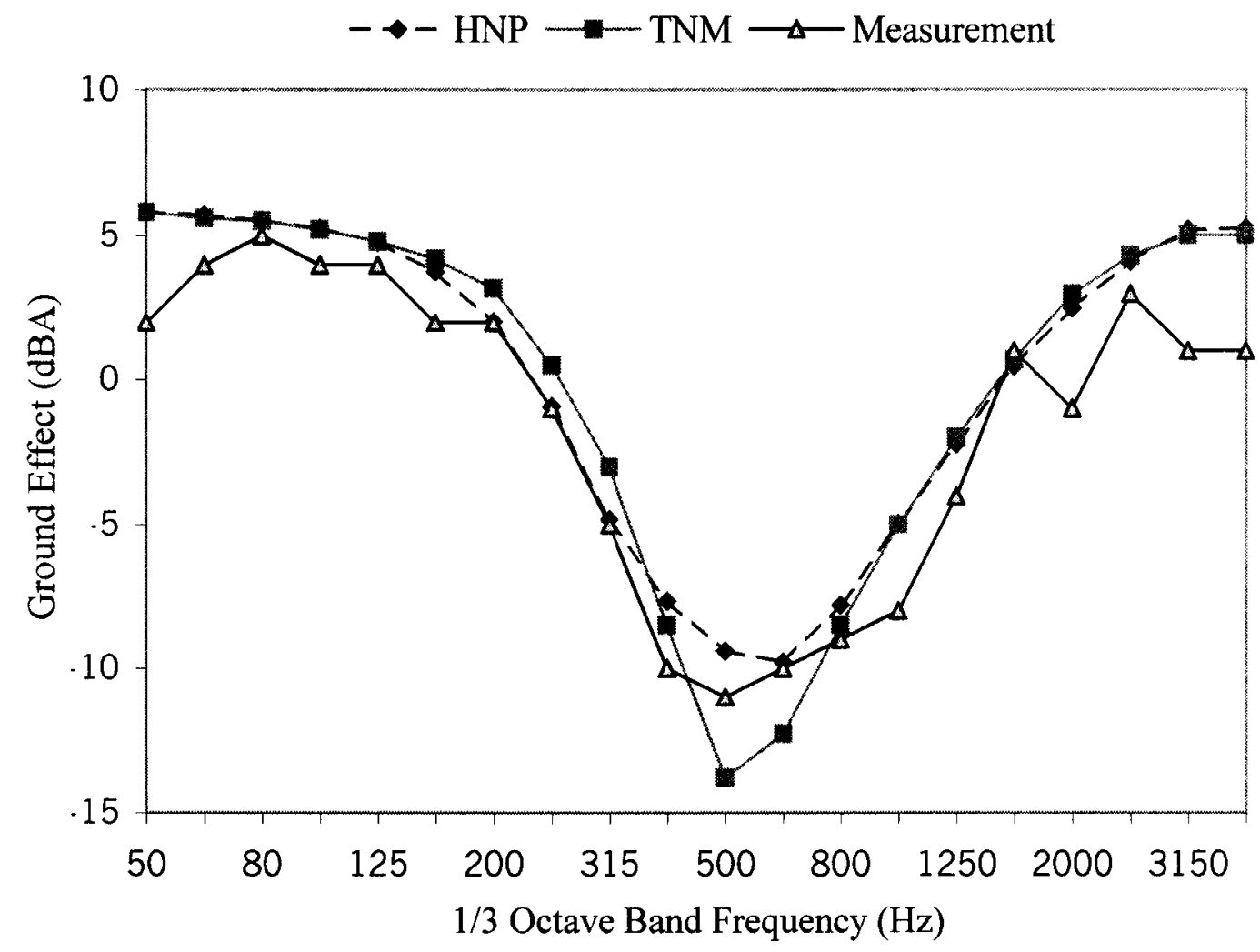

Figure 7-5. Comparison with the measurement by TNM and HNP over grassland, source receiver distance $110 \mathrm{~m}(360 \mathrm{ft})$

As can be seen in Figure 7-5, TNM and HNP demonstrate almost the same ground effect in the high frequency bands from $1000 \mathrm{~Hz}$ and in low frequency bands up to $315 \mathrm{~Hz}$. In the middle frequency bands, both models predict the exact frequency for the first ground effect dip, which occurs at $500 \mathrm{~Hz}$. However, TNM has an error by 4.4 $\mathrm{dBA}$ at this ground effect dip compared to the measurement data, and HNP has an error 
by $1.6 \mathrm{dBA}$, based on the assumption that the measurements of the ground effect are correct.

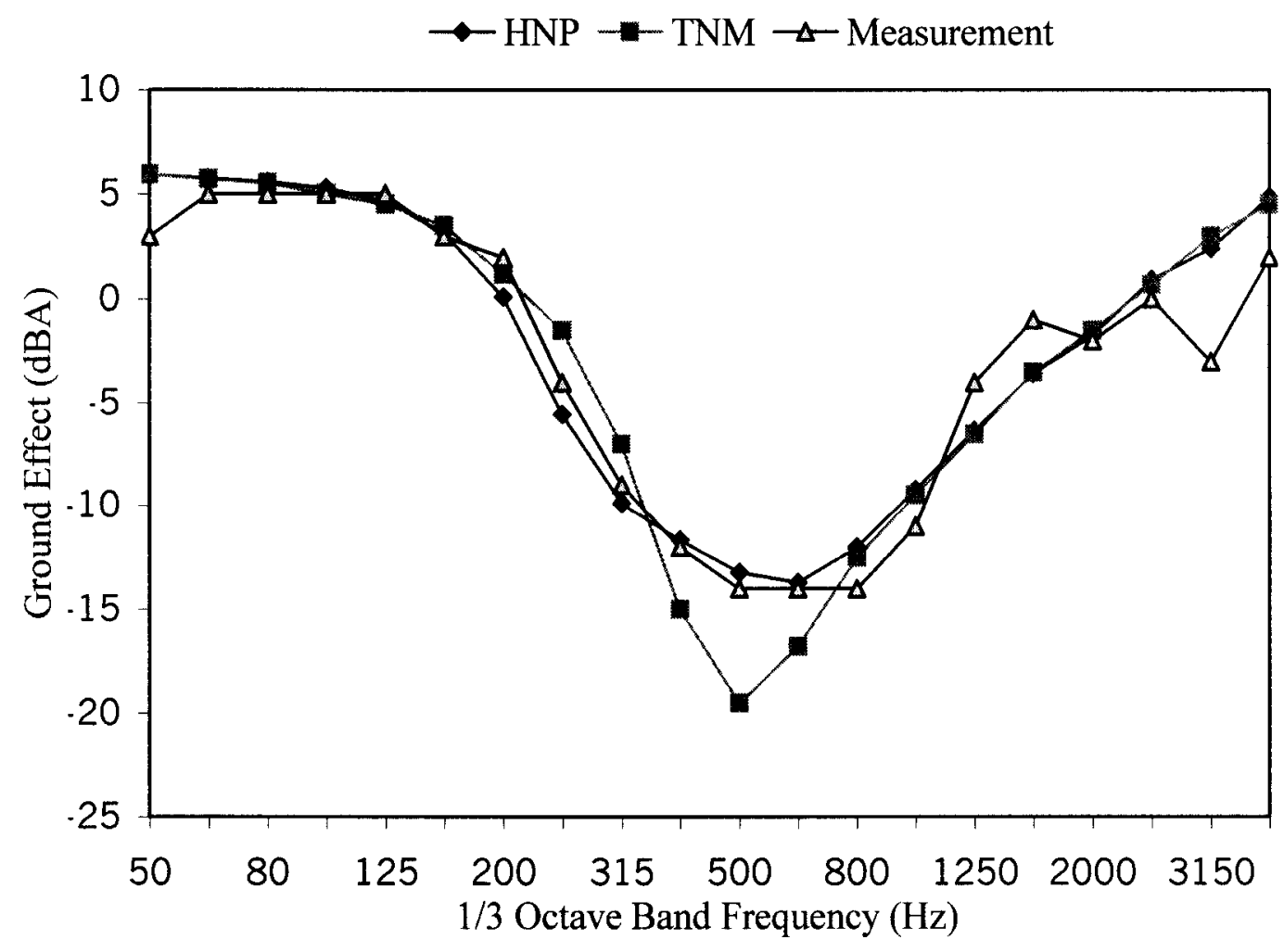

Figure 7-6. Comparison with the measurement by TNM and HNP over grassland, source receiver distance $195 \mathrm{~m}(640 \mathrm{ft})$

As can be seen in Figure 7-6, TNM and HNP demonstrate almost the same ground effect in high frequency from $1000 \mathrm{~Hz}$ and in low frequency bands up to $200 \mathrm{~Hz}$, HNP is in very good agreement with the measured data. In the middle frequency bands, both models predict the exact frequency for the first ground effect dip, which occurs at $500 \mathrm{~Hz}$. However, TNM has a large error by $5.5 \mathrm{dBA}$ at this ground effect dip compared to the measurement data, and HNP has an error by $0.8 \mathrm{dBA}$, based on the assumption that the measurements of the ground effect are correct. 


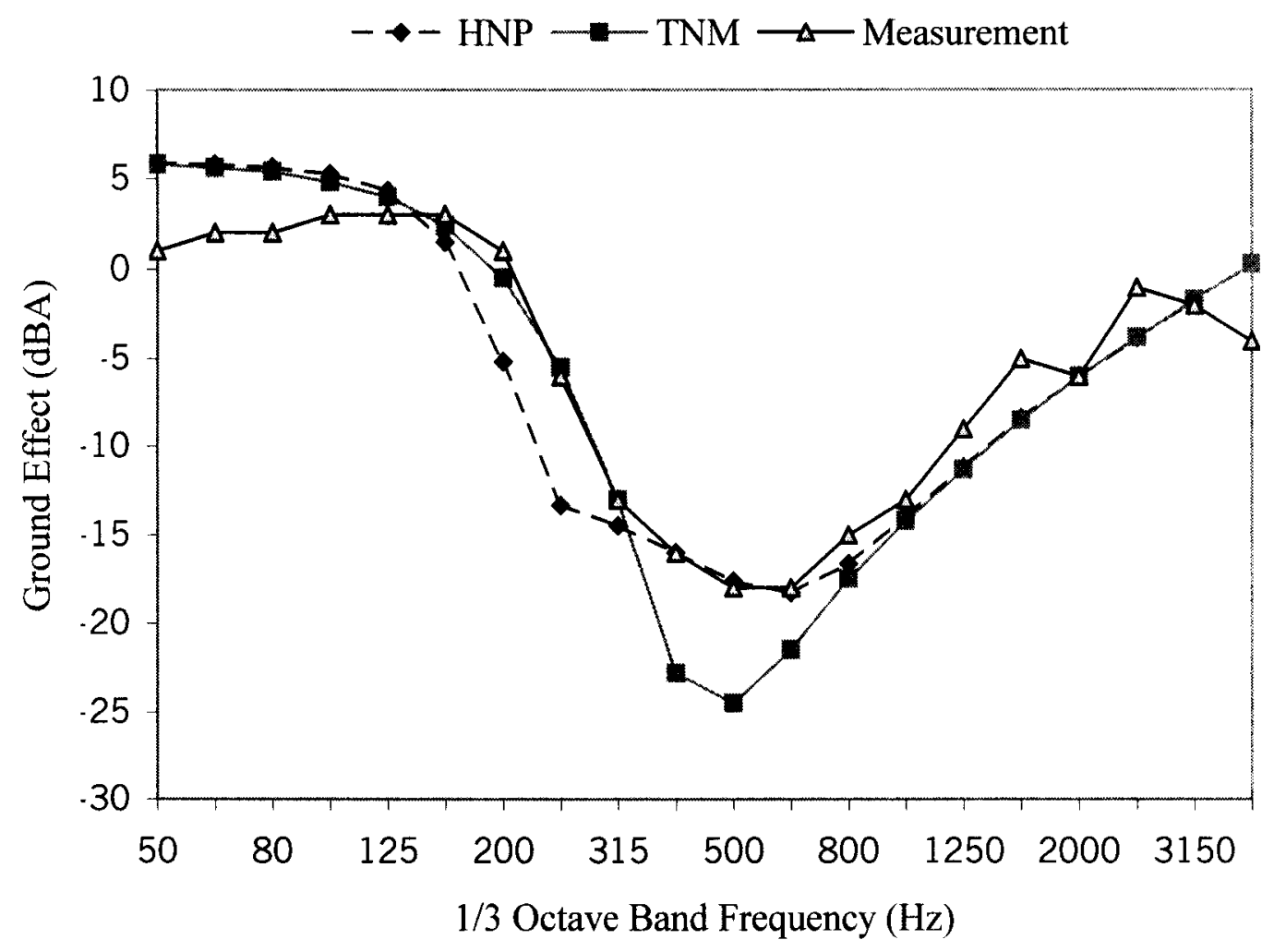

Figure 7-7. Comparison with the measurement by TNM and HNP over grassland, source receiver distance $348 \mathrm{~m}(1140 \mathrm{ft})$

As can be seen in Figure 7-7, TNM and HNP demonstrate almost the same ground effect in high frequency bands from $1000 \mathrm{~Hz}$ and in low frequency bands up to $160 \mathrm{~Hz}$. In the middle frequency bands, both models predict the exact frequency for the first ground effect dip, which occurs at $500 \mathrm{~Hz}$. However, TNM has a large error by 6.5 $\mathrm{dBA}$ at this ground effect dip compared to the measurement data, and HNP has an error by $0.4 \mathrm{dBA}$, based on the assumption that the measurements of the ground effect are correct. 


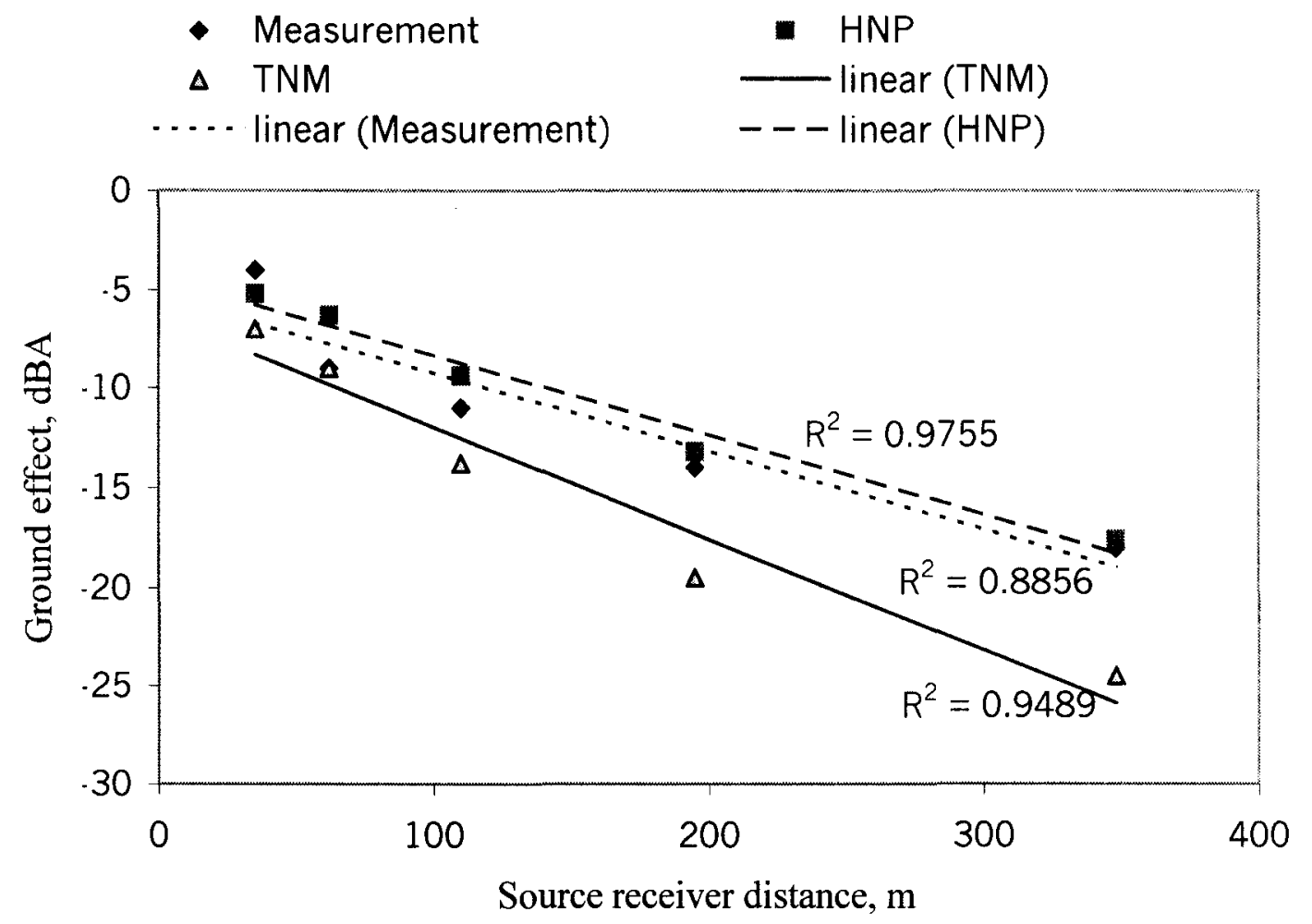

Figure 7-8. Best fit method for TNM, HNP and measurements at the ground effect dip for ground effect

Overall, best fit for linear trends was used for these three sets of data:

measurements, TNM predicted data, and HNP predicted data, as shown in Figure 7-8.

Linear trend for the HNP predicted values shows an excellent fit with $R^{2}=0.98$, Linear

trend for TNM predicted values shows a pretty good relationship with $R^{2}=0.95$, and

linear trend for measurement data shows a fairly good relationship with $R^{2}=0.89$.

Table 7-1.

Difference between mean measured and predicted sound levels at the ground effect dip for ground effect

\begin{tabular}{lllllll}
\hline \multirow{2}{*}{ Model } & \multicolumn{5}{c}{ Difference in Mean, $L_{\text {Aeqh }}(\mathrm{dBA})$} \\
\cline { 2 - 7 } & $35 \mathrm{~m}$ & $62 \mathrm{~m}$ & $110 \mathrm{~m}$ & $195 \mathrm{~m}$ & $348 \mathrm{~m}$ & Average \\
\hline TNM & 3 & 0 & 2.8 & 5.5 & 6.5 & 3.56 \\
HNP & 1.2 & -2.7 & -1.6 & -0.8 & -0.4 & -0.86 \\
\hline
\end{tabular}


As a preliminary analysis tool, the mean differences between the measured and the predicted sound levels at each receiver distance for each model combination are calculated, as shown in Table 7-1. The average difference for TNM is $3.56 \mathrm{dBA}$, and the average difference for $\mathrm{HNP}$ is less than $1 \mathrm{dBA}$. It means that $\mathrm{HNP}$ predicts the ground better than TNM compared with the measurements, and TNM 2.5 has a large error at the ground effect dip for a point source. The trends also show that the further distance the receiver is away from the point source, the greater the difference that occurs between the measured and the predicted ground effect by TNM.

A pair two-tailed t-test is used to compare whether the predicted ground effect of TNM and HNP at the ground effect dip is significantly different from the measured ground effect for all the distances. The summary of t-values is shown in Table 7-2.

Table 7-2. Two-tailed t-test for TNM and HNP at the ground effect dip for ground effect

\begin{tabular}{ll}
\hline & Calculated t-Value \\
\hline Data source & Average \\
Tabulated t $5 \%$ & 2.776 \\
TNM & 3.124 \\
HNP & 1.328 \\
\hline
\end{tabular}

The results contained in Table 7-2 indicate that there is no statistically significant difference between the measured ground effect and the predicted ground effect by HNP for all the distances up to $348 \mathrm{~m}$ ( $1140 \mathrm{ft}$ ) from the point source at a $95 \%$ confidence level. On the other hand, the measured and the predicted ground effect by TNM indicate that the difference is statistically significant, and this difference is caused by some factors 
other than chance. From the analysis in chapter IV, this difference may be due to the following reasons:

- When calculating $w$, the numerical distance, TNM used the improper parameter, $r$, the total distance between the source and the receiver. According to the original papers (Chessell, 1977), (Jonasson, 1972), $r^{\prime}$, the total distance between the image source and the receiver, should be used instead of $r$.

- When calculating the ground wave function $F(w)$, TNM utilizes a simplified asymptotic equation; however, this may induce errors when calculating the ground attenuation. See Chapter IV for detail.

\subsubsection{Comparisons of Barrier Insertion Loss}

The complete HNP reflection and diffraction models are applied to compare with the barrier insertion loss measurements of Scholes (Scholes, 1971) and the predicted TNM insertion loss. The edge diffraction model proposed by De Jong is not used here since there was no impedance discontinuity between the point source (speaker) and the receiver over the flat grassland measured by Scholes. The barrier insertion loss measurements were also conducted at the Hatfield site. Numerous measurements were conducted at different source-receiver distances, with different barrier and receiver heights, with some receivers located in the shadow zone and others located in the illuminated zone. Measurements under different meteorological conditions were conducted. The comparisons with TNM and HNP are made with a limited set of the measured data, since both models do not take meteorological conditions into account except for atmospheric absorption. Therefore, neutral meteorological conditions with 
zero vector wind are selected for verification. Comparisons are made by HNP with the published data contained in the TNM Technical Manual with the following geometry:

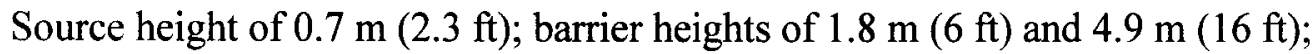
microphone heights of $1.5 \mathrm{~m}(5 \mathrm{ft}), 3 \mathrm{~m}(10 \mathrm{ft}), 6 \mathrm{~m}(20 \mathrm{ft})$, and $12 \mathrm{~m}(40 \mathrm{ft})$; horizontal

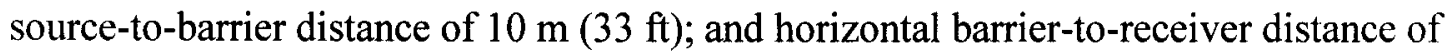
$30 \mathrm{~m}(100 \mathrm{ft})$. For a direct comparison with the measured data and the TNM predicted data, the HNP's $1 / 3$-octave band values are combined (energy sum prior to subtraction) to obtain the octave-band values. An EFR of 325 cgs Rayls is assumed for the grassland at Hatfield site. Figures 7-9 through 7-16 present the barrier insertion loss comparisons.

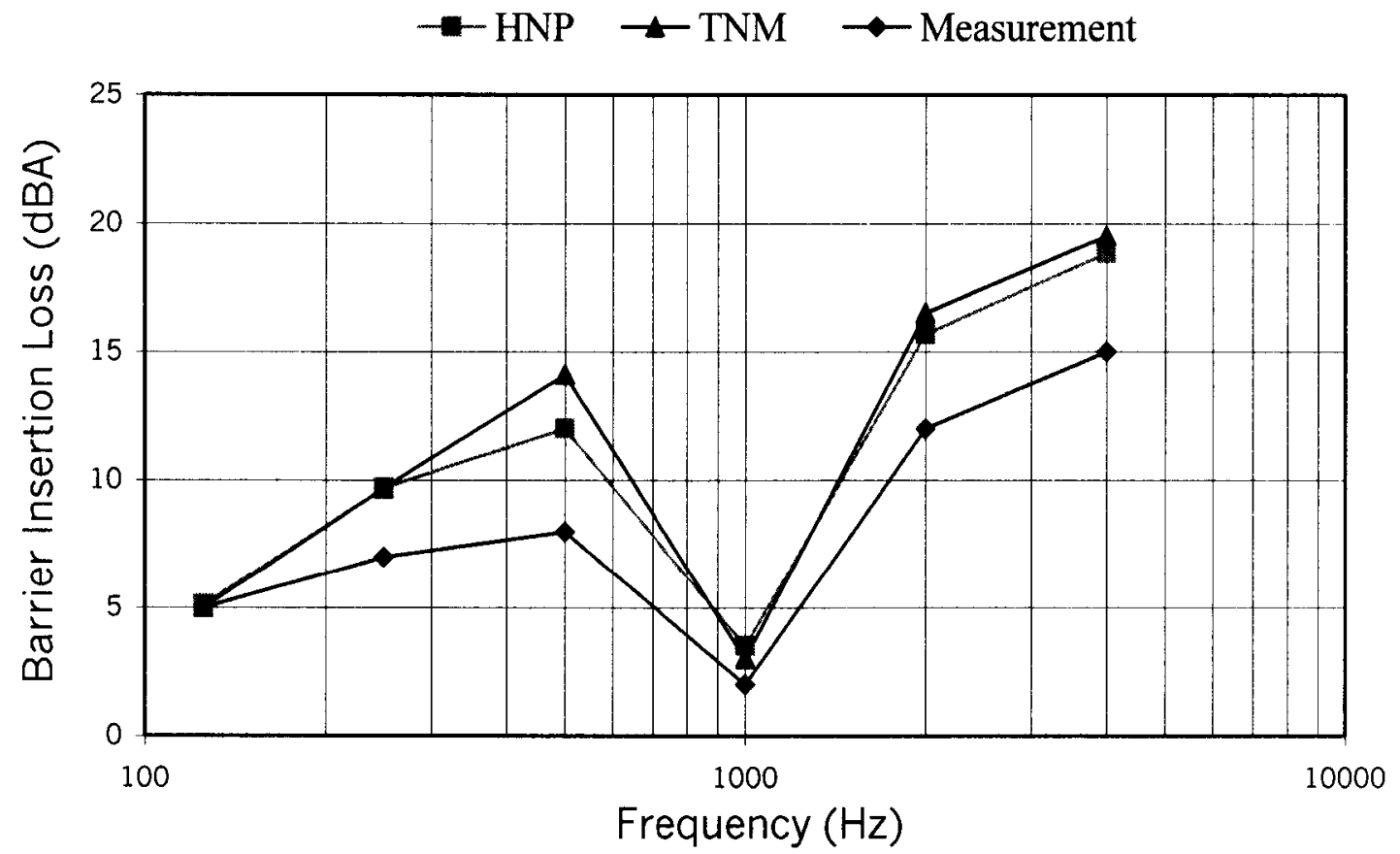

Figure 7-9. Comparison of barrier insertion loss in octave bands, source height $0.7 \mathrm{~m}$, receiver height $1.5 \mathrm{~m}$, barrier height $1.8 \mathrm{~m}$

As can be seen in Figure 7-9, TNM and HNP demonstrate similar barrier insertion loss in all frequency bands. Both models predict the exact frequency for the first minimum insertion loss in the middle frequency band, which occurs at $1000 \mathrm{~Hz}$. The 
difference of insertion loss at this minimum insertion loss between TNM and the measurement, $\mathrm{HNP}$ and the measurement are less than $1.5 \mathrm{dBA}$.

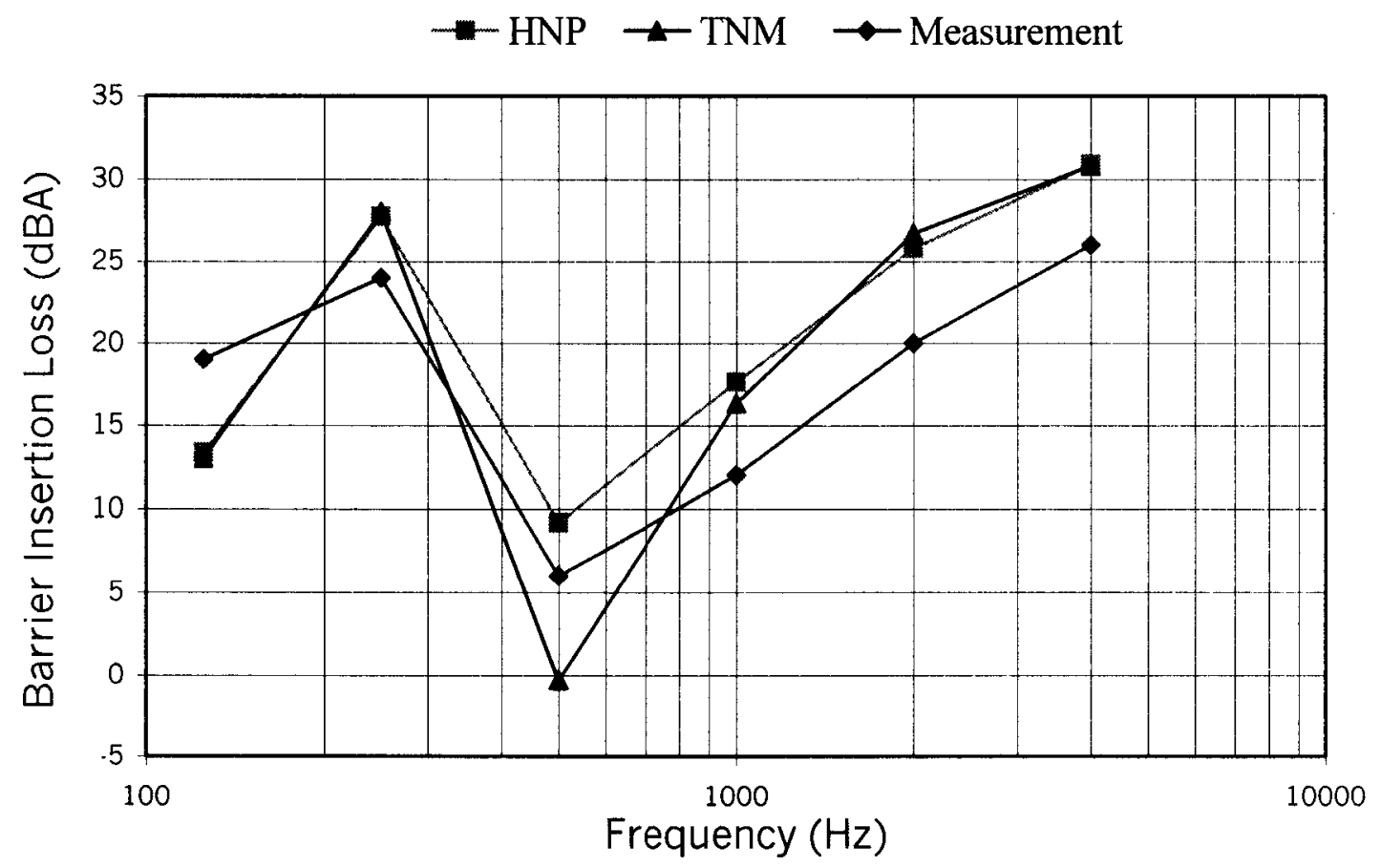

Figure 7-10. Comparison of barrier insertion loss in octave bands, source height $0.7 \mathrm{~m}$, receiver height $1.5 \mathrm{~m}$, barrier height $4.9 \mathrm{~m}$

As can be seen in Figure 7-10, TNM and HNP demonstrate almost the same insertion loss in high frequency bands from $1000 \mathrm{~Hz}$ to $4000 \mathrm{~Hz}$ and in low frequency bands from $125 \mathrm{~Hz}$ to $250 \mathrm{~Hz}$. In the middle frequency bands, both models predict the exact frequency for the first minimum insertion loss, which occurs at $500 \mathrm{~Hz}$. However, TNM demonstrates a large error of insertion loss at this frequency by $6.3 \mathrm{dBA}$ compared to the measurement data, and HNP tends to reduce the error to $3.2 \mathrm{dBA}$.

As can be seen in Figure 7-11, TNM and HNP demonstrate almost the same insertion loss in high frequency bands from $1000 \mathrm{~Hz}$ to $2000 \mathrm{~Hz}$ and in low frequency bands from $125 \mathrm{~Hz}$ to $250 \mathrm{~Hz}$. In the middle frequency bands, both models predict the exact frequency for the minimum insertion loss, which occurs at $500 \mathrm{~Hz}$. However, TNM 
demonstrates a large error of insertion loss at this critical frequency by $3 \mathrm{dBA}$ compared to the measurement data, and HNP tends to reduce the error to $0.4 \mathrm{dBA}$.

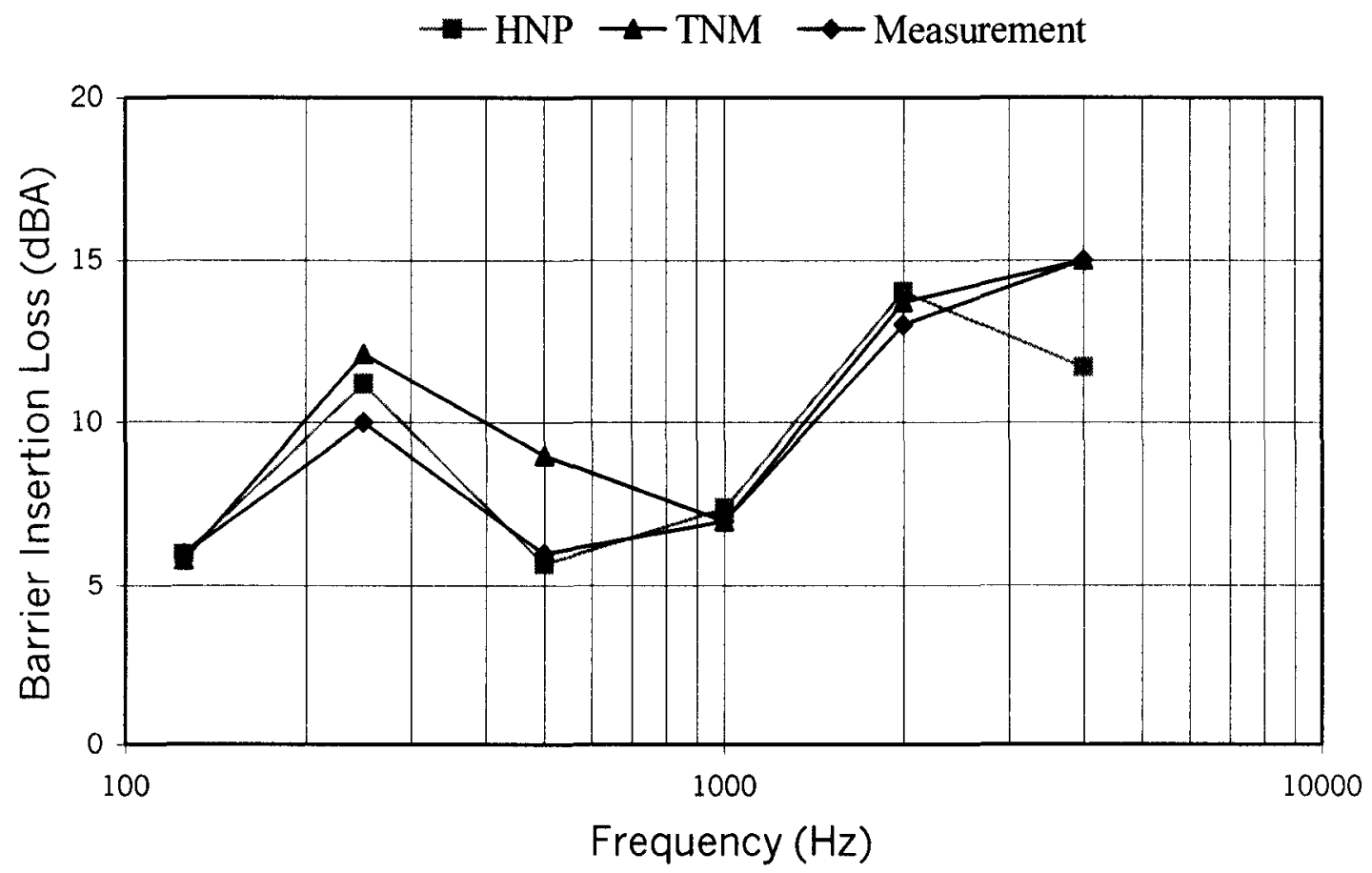

Figure 7-11. Comparison of barrier insertion loss in octave bands, source height $0.7 \mathrm{~m}$, receiver height $3 \mathrm{~m}$, barrier height $1.8 \mathrm{~m}$

$\rightarrow-\mathrm{HNP} \rightarrow$ TNM $\rightarrow$ Measurement

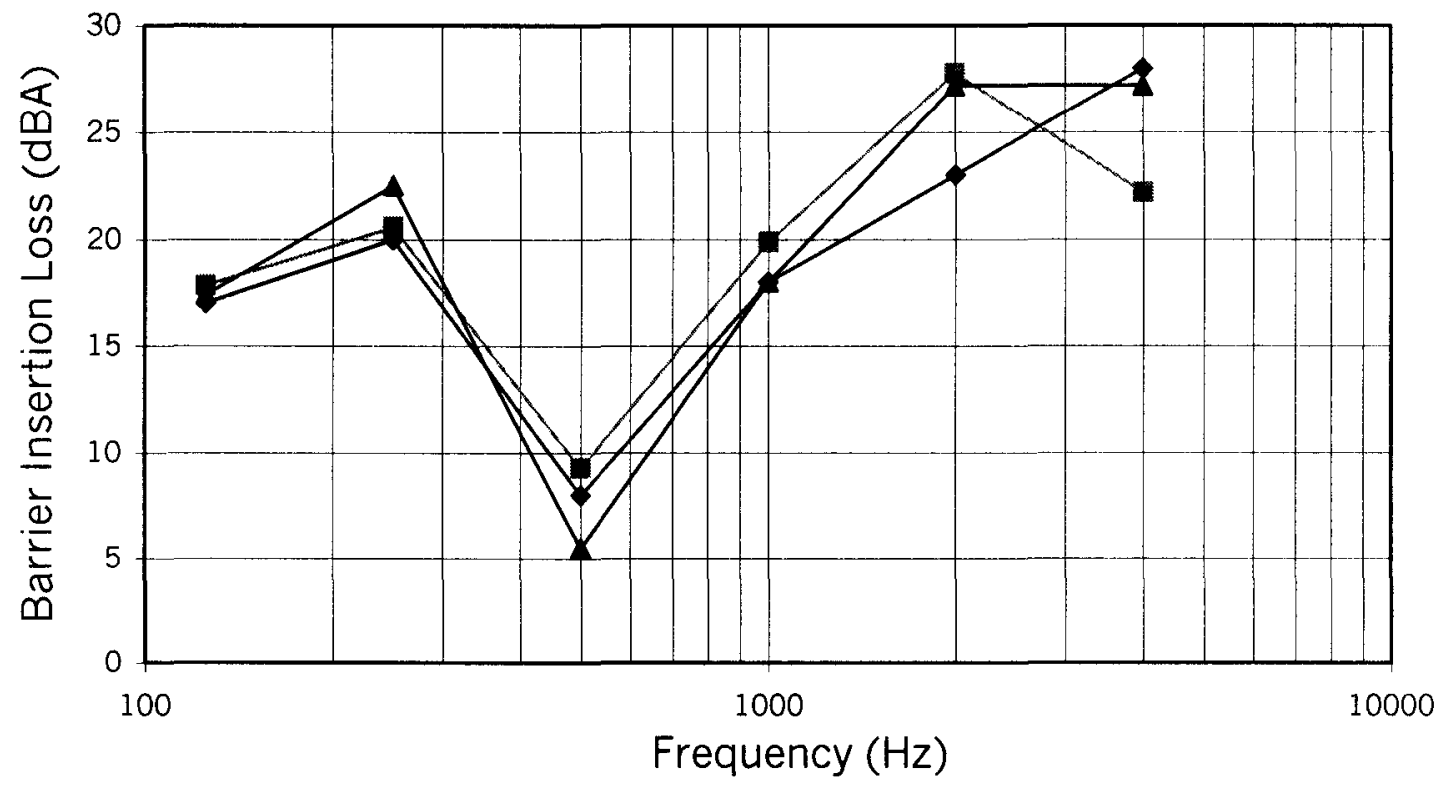

Figure 7-12. Comparison of barrier insertion loss in octave bands, source height $0.7 \mathrm{~m}$, receiver height $3 \mathrm{~m}$, barrier height $4.9 \mathrm{~m}$ 
As can be seen in Figure 7-12, TNM and HNP demonstrate almost the same insertion loss in high frequency bands from $1000 \mathrm{~Hz}$ to $2000 \mathrm{~Hz}$ and in low frequency bands from $125 \mathrm{~Hz}$ to $250 \mathrm{~Hz}$. In the middle frequency bands, both models predict the exact frequency for the minimum insertion loss, which occurs at $500 \mathrm{~Hz}$. However, TNM has an error of insertion loss by $2.5 \mathrm{dBA}$ at this critical frequency compared to the measurement data, and HNP tends to reduce the error to $1.3 \mathrm{dBA}$.

As can be seen in Figure 7-13, TNM demonstrates the better prediction of insertion loss in high frequency bands from $1000 \mathrm{~Hz}$ to $4000 \mathrm{~Hz}$ and HNP predicts better results in low frequency bands from $125 \mathrm{~Hz}$ to $250 \mathrm{~Hz}$. In the middle frequency bands, both models predict the exact frequency for the minimum insertion loss, which occurs at $500 \mathrm{~Hz}$. However, TNM has a large error of insertion loss at this critical frequency by 11.5 dBA compared to the measurement data, and HNP tends to reduce the error to 0.7 dBA.

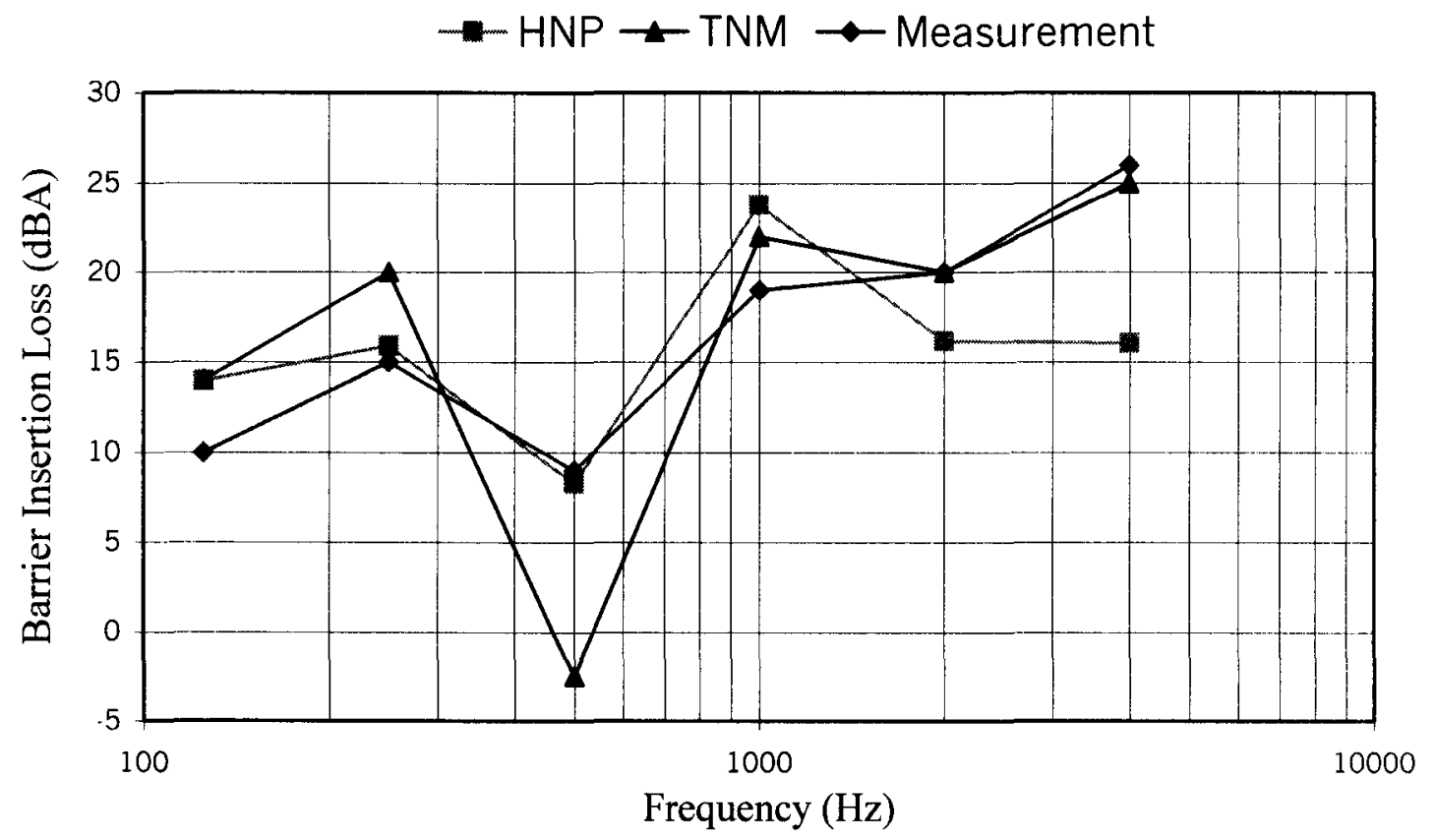

Figure 7-13. Comparison of barrier insertion loss in octave bands, source height $0.7 \mathrm{~m}$, receiver height $6 \mathrm{~m}$, barrier height $4.9 \mathrm{~m}$ 


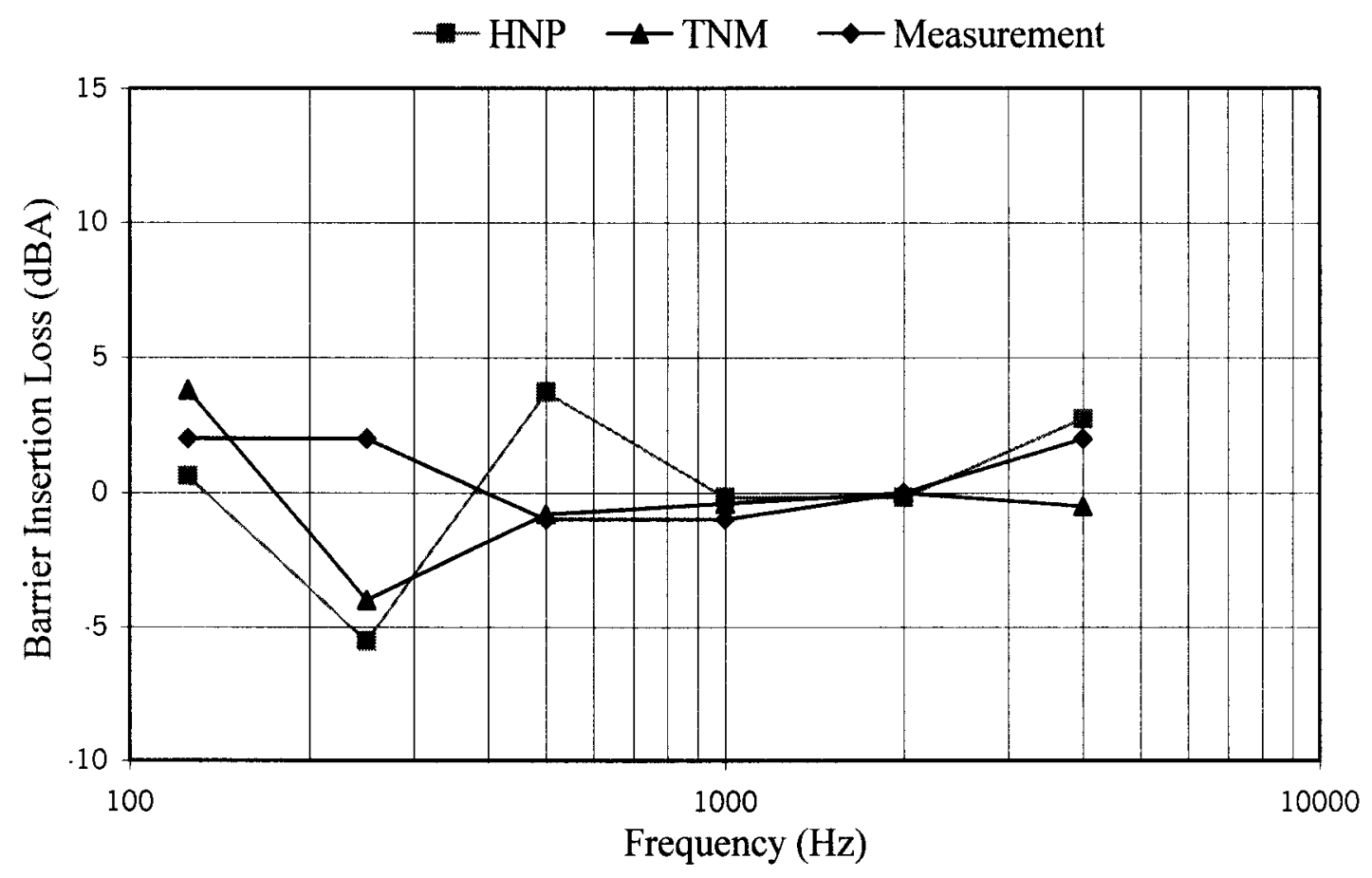

Figure 7-14. Comparison of barrier insertion loss in octave bands, source height $0.7 \mathrm{~m}$, receiver height $12 \mathrm{~m}$, barrier height $1.8 \mathrm{~m}$

As can be seen in Figure 7-14, TNM and HNP demonstrate similar insertion loss in high frequency bands from $1000 \mathrm{~Hz}$ to $2000 \mathrm{~Hz}$. However, both models predict differently at the frequency of $125 \mathrm{~Hz}, 500 \mathrm{~Hz}$ and $4000 \mathrm{~Hz}$. For example, TNM is in pretty good agreement with the measurement at the frequency of $500 \mathrm{~Hz}$ and $\mathrm{HNP}$ has an error of $4.7 \mathrm{dBA}$. At the frequency of $4000 \mathrm{~Hz}$, TNM shows an error of $2.5 \mathrm{dBA}$ and the error for HNP is within $1.0 \mathrm{dBA}$.

As can be seen in Figure 7-15, TNM and HNP demonstrate almost the same insertion loss in high frequency bands from $2000 \mathrm{~Hz}$ to $4000 \mathrm{~Hz}$ and in low frequency bands at $125 \mathrm{~Hz}$. However, TNM differs largely from the measurements at frequency 250 $\mathrm{Hz}, 500 \mathrm{~Hz}$, and $1000 \mathrm{~Hz}$, with an error of $3 \mathrm{dBA}$ at $250 \mathrm{~Hz}, 7.5 \mathrm{dBA}$ at $500 \mathrm{~Hz}$, and 5.3 $\mathrm{dBA}$ at $1000 \mathrm{~Hz}$. HNP predicts better insertion loss than TNM at these frequency bands, with an error of $1 \mathrm{dBA}$ at $250 \mathrm{~Hz}, 4.9 \mathrm{dBA}$ at $500 \mathrm{~Hz}$, and $0.7 \mathrm{dBA}$ at $1000 \mathrm{~Hz}$. 


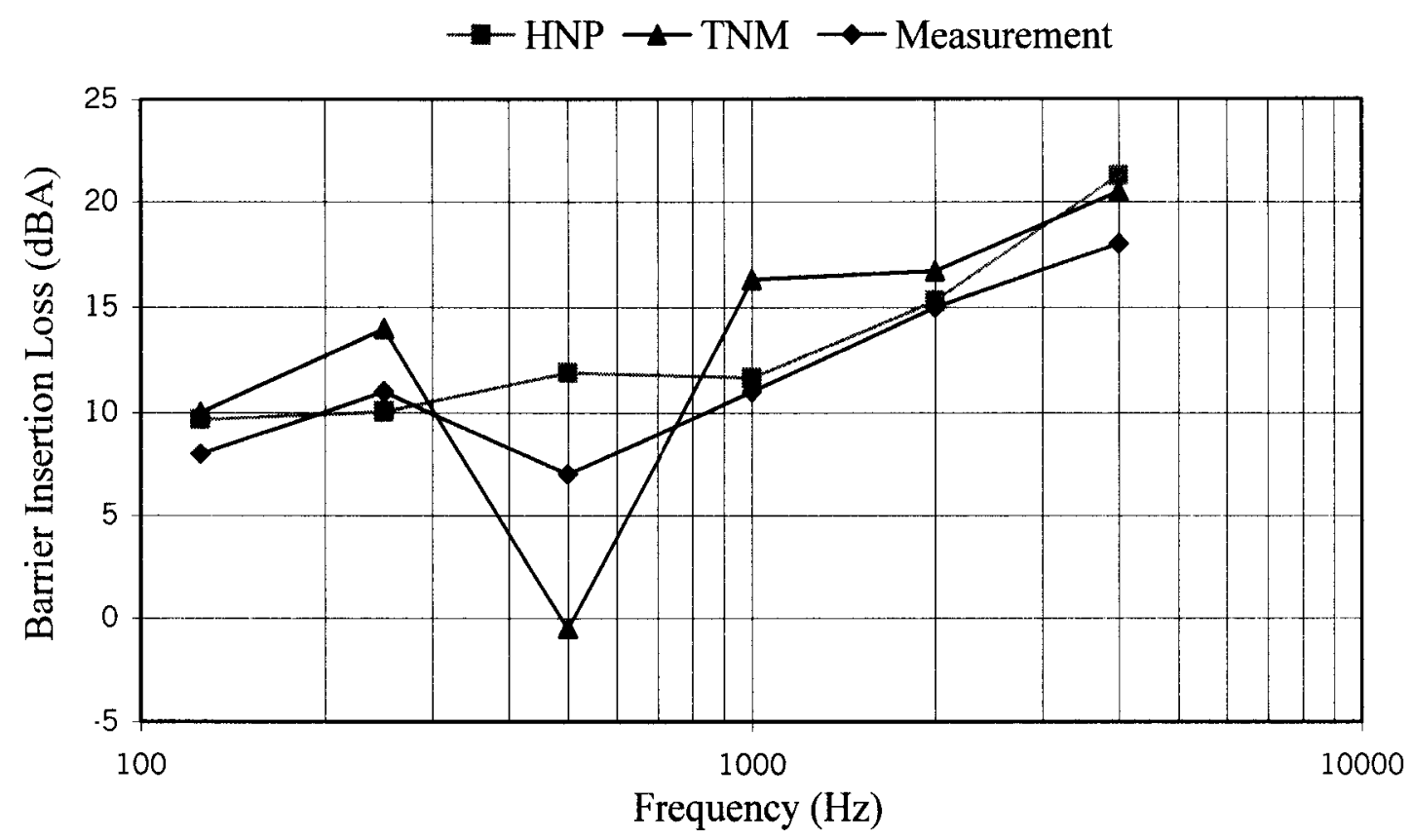

Figure 7-15. Comparison of barrier insertion loss in octave bands, source height $0.7 \mathrm{~m}$, receiver height $12 \mathrm{~m}$, barrier height $4.9 \mathrm{~m}$

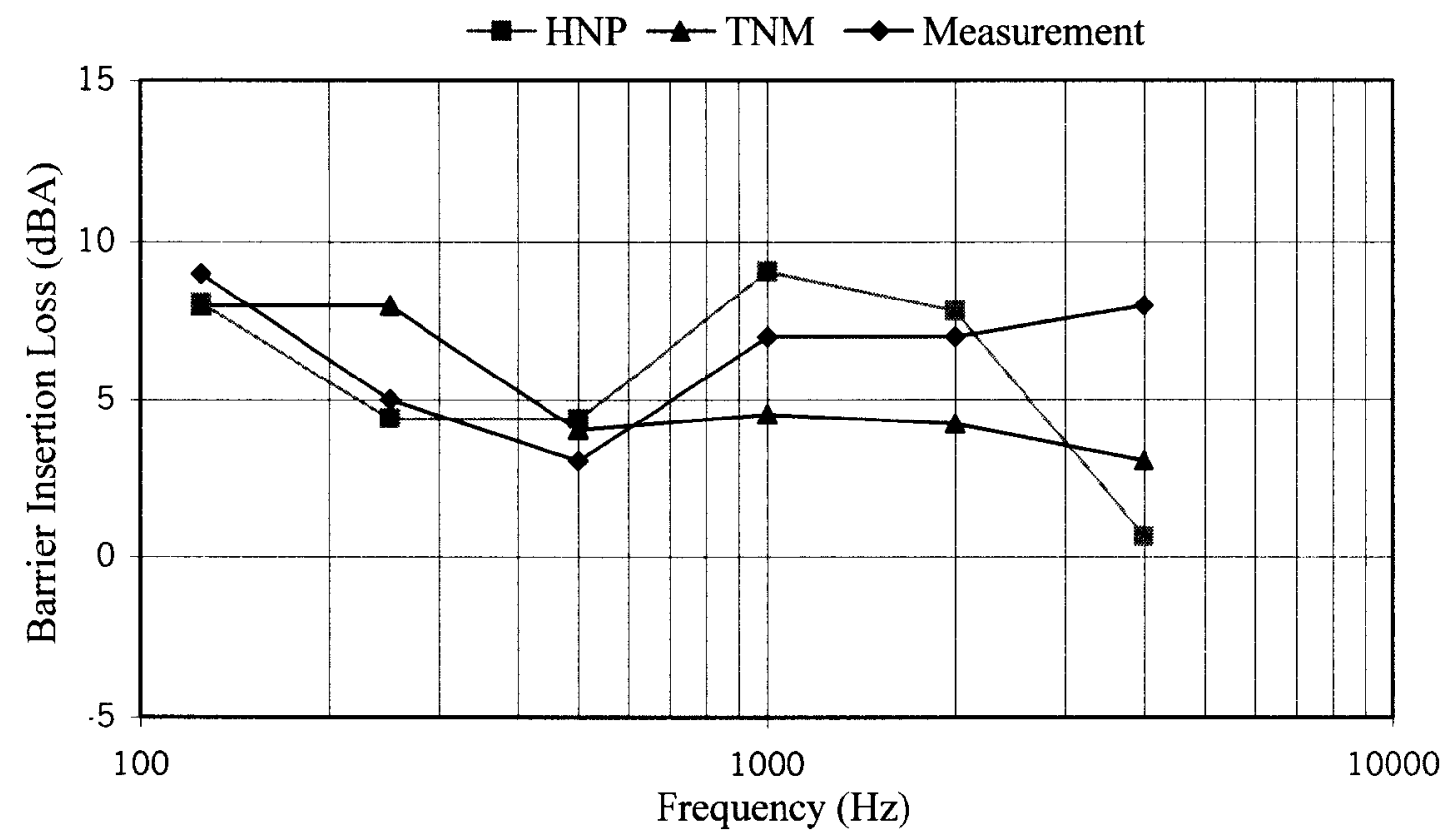

Figure 7-16. Comparison of barrier insertion loss in octave bands, source height $0.7 \mathrm{~m}$, receiver height $6 \mathrm{~m}$, barrier height $1.8 \mathrm{~m}$

As can be seen in Figure 7-16, TNM and HNP demonstrate the similar insertion loss at the frequency of $125 \mathrm{~Hz}$ and $500 \mathrm{~Hz}$. In the middle frequency bands, both models 
predict the exact frequency for the minimum insertion loss, which occurs at $500 \mathrm{~Hz}$. The error at this frequency is $1.0 \mathrm{dBA}$ for TNM and $1.3 \mathrm{dBA}$ for HNP. TNM differs largely from $\mathrm{HNP}$ and measurement at frequency $250 \mathrm{~Hz}, 1000 \mathrm{~Hz}, 2000 \mathrm{~Hz}$ and $4000 \mathrm{~Hz}$, with an error of $3.0 \mathrm{dBA}$ at $250 \mathrm{~Hz}, 2.5 \mathrm{dBA}$ at $1000 \mathrm{~Hz}, 2.8 \mathrm{dBA}$ at $2000 \mathrm{~Hz}$ and $5.0 \mathrm{dBA}$ at $4000 \mathrm{~Hz}$ compared with the measurement. $\mathrm{HNP}$ has an error of $0.6 \mathrm{dBA}$ at $250 \mathrm{~Hz}, 2.1$ dBA at $1000 \mathrm{~Hz}, 0.8 \mathrm{dBA}$ at $1000 \mathrm{~Hz}$ and $7.4 \mathrm{dBA}$ at $4000 \mathrm{~Hz}$ compared with the measurement.

\subsubsection{Descriptive Statistics}

Figure 7-17 shows the comparisons of predicted insertion loss with the measurements at the minimum insertion loss for the above eight scenarios. Descriptive statistical methods including mean error, standard deviation, error range, minimum error, and maximum error are selected to test the prediction accuracy of insertion loss by HNP and TNM, shown in Table 7-3.

Table 7-3.

Descriptive statistics for comparison of predicted insertion loss at frequency dip by HNP and TNM

\begin{tabular}{lll}
\hline & HNP & TNM \\
\hline Mean & 2.25 & 4.13 \\
Standard Deviation & 1.79 & 3.95 \\
Range & 4.6 & 11.3 \\
Minimum & 0.3 & 0.2 \\
Maximum & 4.9 & 11.5 \\
\hline
\end{tabular}




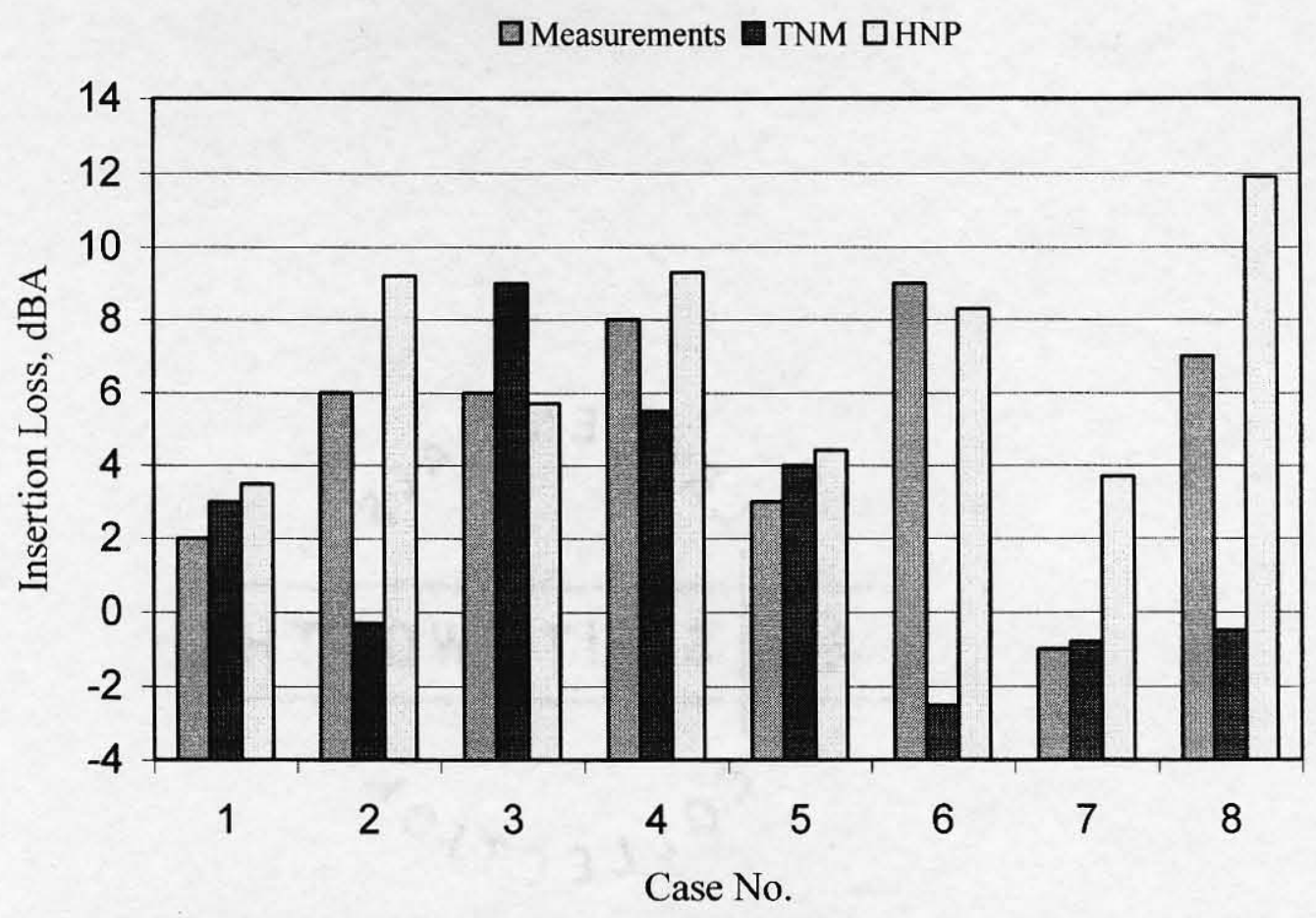

Figure 7-17. Comparison of predicted insertion loss with the measurements at minimum insertion loss by TNM and HNP

Table 7-3 demonstrates that HNP performs the best in four out of the five statistical categories evaluated. TNM has the best value for minimum error of $0.2 \mathrm{dBA}$, versus $0.3 \mathrm{dBA}$ for $\mathrm{HNP}$. It can be seen that, on average, $\mathrm{HNP}$ has an error of $2.25 \mathrm{dBA}$ while TNM is $4.13 \mathrm{dBA}$. TNM shows a large standard deviation of $3.95 \mathrm{dBA}$, and it is $1.79 \mathrm{dBA}$ for HNP. Overall, it can be concluded that HNP predicts the insertion loss better than TNM compared to the measurements for a point source at the minimum insertion loss.

\subsection{Single Line Source Verification}

In this section, insertion loss will be compared by HNP 1.0 and TNM 2.5 for a single lane roadway. The geometry configurations are as follows: length of single lane roadway $610 \mathrm{~m}(2000 \mathrm{ft})$, width of single lane roadway $3.66 \mathrm{~m}(12 \mathrm{ft})$, traffic volume 
1000 autos, $60 \mathrm{~m} / \mathrm{h}, 100 \mathrm{HT}, 55 \mathrm{~m} / \mathrm{h}$, roadway to barrier distance $15 \mathrm{~m}(50 \mathrm{ft})$, barrier height $4.5 \mathrm{~m}(15 \mathrm{ft})$, receiver distances $15 \mathrm{~m}(50 \mathrm{ft}), 30 \mathrm{~m}(100 \mathrm{ft}), 61 \mathrm{~m}(200 \mathrm{ft})$, and 122 $\mathrm{m}(400 \mathrm{ft})$ from barrier, receiver height $1.5 \mathrm{~m}(5 \mathrm{ft})$, and the ground type as lawn.

Table 7-4.

The predicted insertion loss (dBA) of a one-lane line source by TNM 2.5, STAMINA 2.0 , and HNP 1.0 with varying distance

\begin{tabular}{llll}
\hline Mic. Location & TNM 2.5 & STAMINA 2.0 & HNP 1.0 \\
\hline $50 \mathrm{ft}$ & 11.1 & 9.9 & 9.9 \\
$100 \mathrm{ft}$ & 9.3 & 8.0 & 8.3 \\
$200 \mathrm{ft}$ & 7.5 & 6.6 & 7.0 \\
$400 \mathrm{ft}$ & 6.6 & 4.3 & 6.2 \\
\hline
\end{tabular}

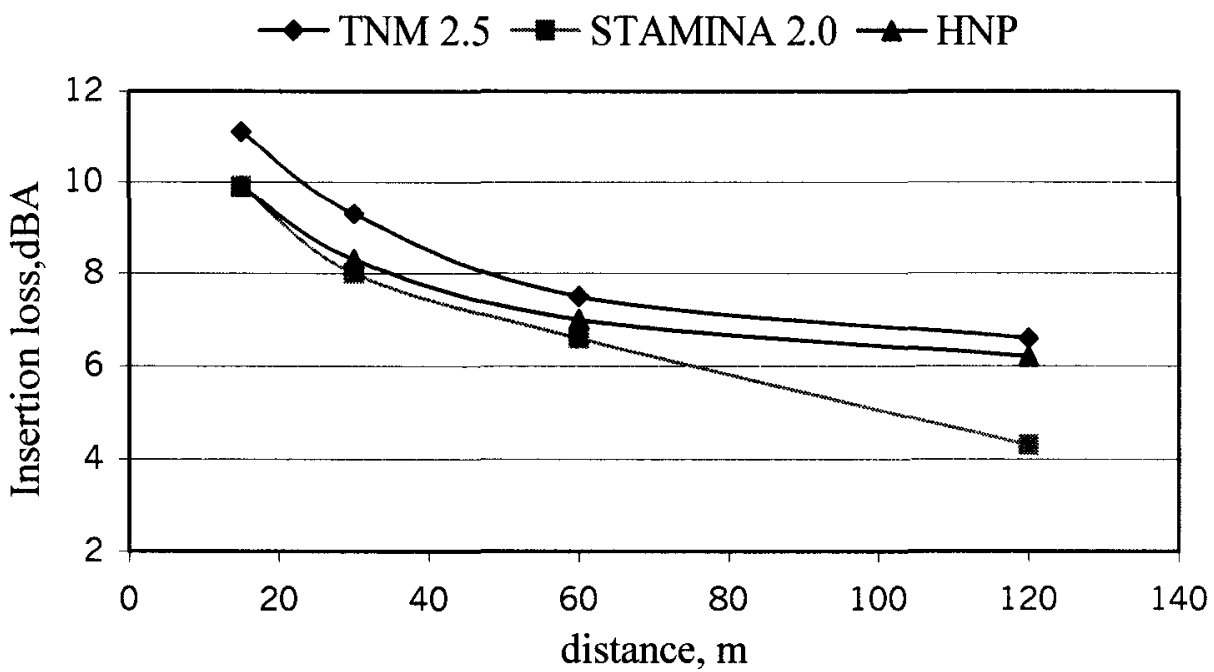

Figure 7-18. Plot for the predicted insertion loss of a one-lane line source by TNM 2.5, STAMINA 2.0, and HNP 1.0 with varying distance

As can be seen in Table 7-4 and Figure 7-18, TNM 2.5 over-predicts the insertion loss compared to STAMINA 2.0 and HNP 1.0, and STAMINA 2.0 under-predicts the insertion loss compared to HNP 1.0 for a single line source. The predicted insertion loss ranges from $6.6 \mathrm{dBA}$ to $11.1 \mathrm{dBA}$ for TNM 2.5. The predicted insertion loss ranges from 
$4.3 \mathrm{dBA}$ to $9.9 \mathrm{dBA}$ for STAMINA 2.0 and the predicted insertion loss ranges from 6.2 dBA to $9.9 \mathrm{dBA}$ for HNP 1.0 .

\subsection{Multiple Line Source Verification}

In this section, insertion loss will be compared by HNP and TNM for a four-lane roadway. The geometry configurations are as follows: length of the four-lane roadway

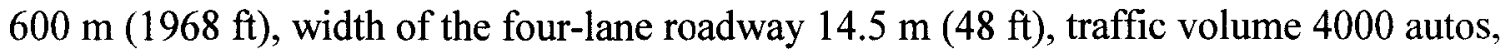
$60 \mathrm{~m} / \mathrm{h}, 400 \mathrm{HT}, 55 \mathrm{~m} / \mathrm{h}$, roadway to barrier distance $15 \mathrm{~m}(50 \mathrm{ft})$, barrier height $4.5 \mathrm{~m}$ $(15 \mathrm{ft})$, receiver distances $15 \mathrm{~m}(50 \mathrm{ft}), 30 \mathrm{~m}(100 \mathrm{ft}), 61 \mathrm{~m}(200 \mathrm{ft})$, and $122 \mathrm{~m}(400 \mathrm{ft})$ from barrier, receiver height $1.5 \mathrm{~m}(5 \mathrm{ft})$, and the ground type as lawn.

As can be seen in Table 7-5 and Figure 7-19, TNM 2.5 over-predicts the insertion loss compared to STAMINA 2.0 and HNP 1.0 and STAMINA 2.0 under-predicts the insertion loss compared to HNP 1.0 for 4-lane line sources. The predicted insertion loss ranges from $7.0 \mathrm{dBA}$ to $12.6 \mathrm{dBA}$ for TNM 2.5. The predicted insertion loss ranges from 4.9 dBA to $9.5 \mathrm{dBA}$ for STAMINA 2.0 and the predicted insertion loss ranges from 5.7 dBA to $10.5 \mathrm{dBA}$ for HNP 1.0 .

Table 7-5.

The predicted insertion loss (dBA) of a four-lane line sources by TNM 2.5, STAMINA 2.0 , and HNP 1.0 with varying distance

\begin{tabular}{llll}
\hline Mic. Location & TNM 2.5 & STAMINA 2.0 & HNP 1.0 \\
\hline $50 \mathrm{ft}$ & 12.6 & 9.5 & 10.5 \\
$100 \mathrm{ft}$ & 11.1 & 7.5 & 8.5 \\
$200 \mathrm{ft}$ & 8.9 & 6.0 & 6.7 \\
$400 \mathrm{ft}$ & 7.0 & 4.9 & 5.7 \\
\hline
\end{tabular}




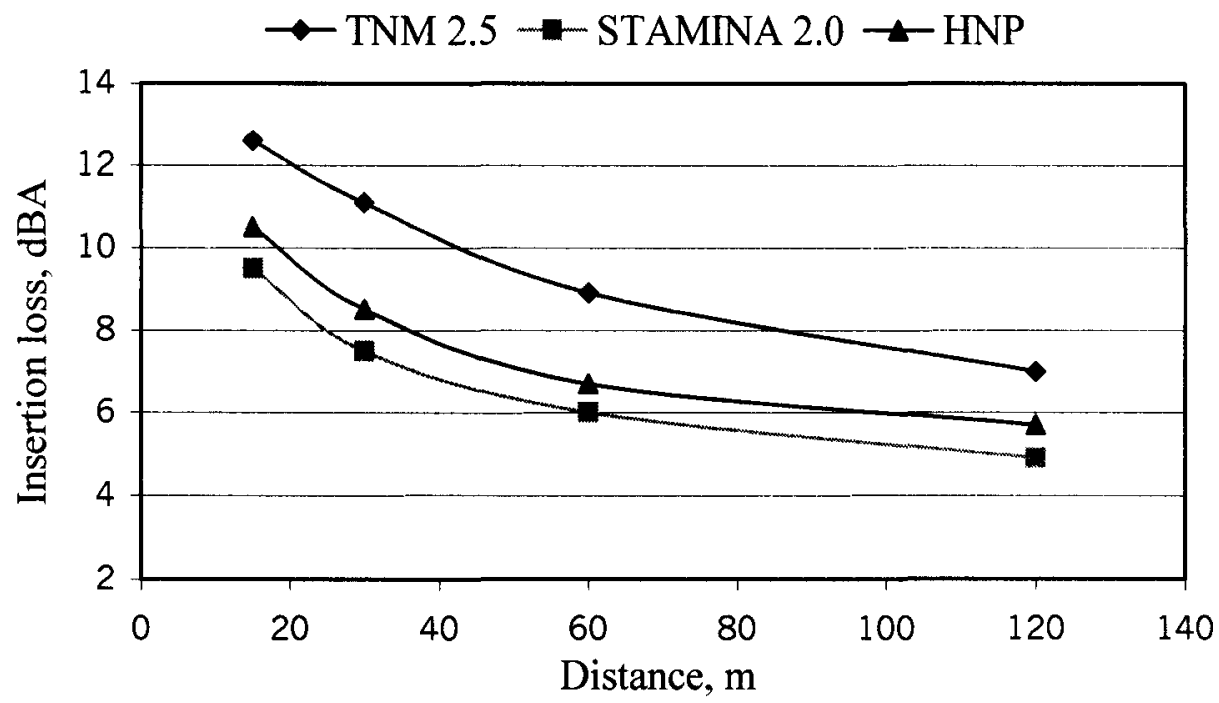

Figure 7-19. Plot for the predicted insertion loss of a four-lane line sources by TNM 2.5, STAMINA 2.0, and HNP 1.0 with varying distance

\subsection{Comparison of Diffraction Theory in TNM 2.5 and STAMINA 2.0}

In this section, comparisons are made to verify the diffraction theory in TNM 2.5 and STAMINA 2.0. TNM adopts De Jong's model, which is based on the MacDonald diffraction theory. As discussed in Chapter IV, according to De Jong's model (1983), the diffracted field at the receiver behind a semi-infinite screen can be expressed as follows:

$$
\begin{aligned}
& p_{d}=\frac{e^{-i \pi / 4}}{\sqrt{\pi}} \frac{e^{i k R}}{k L} F\left(\chi_{-}\right)+\frac{e^{-i \pi / 4}}{\sqrt{\pi}} \frac{e^{i k s}}{k L} F\left(\chi_{+}\right) \\
& \chi_{-}=-\frac{1}{2}\left(\frac{k r_{0} r}{2 L}\right)^{1 / 2} \cos \left(\frac{\phi-\phi_{0}}{2}\right)=\sqrt{k(L-R)} \\
& \chi_{+}=-\frac{1}{2}\left(\frac{k r_{0} r}{2 L}\right)^{1 / 2} \cos \left(\frac{\phi+\phi_{0}}{2}\right)=\sqrt{k(L-S)}
\end{aligned}
$$

where,

$L \quad$ distance of path from the source to the receiver over the barrier

$X_{ \pm}$argument for the relative position of the source, receiver and the edge of barrier

$r_{0} \quad$ distance from the source to the top of the barrier

$r \quad$ distance from the top of the barrier to the receiver 
$R \quad$ direct distance from the source to the receiver

$S \quad$ direct distance from the image source in the barrier to the receiver

Equation (7-1) can be explained that the first term is the contribution of real source and the second term is the contribution of image source. The free field pressure at the same point without the barrier is expressed in Equation (7-4):

$$
P_{\text {free- field }}=\frac{e^{i k R}}{k R}
$$

In order to compute the insertion loss behind the barrier, the ratio of $P_{d}$ and $P_{\text {free_field }}$ is needed and given in Equation (7-5):

Since,

$$
\frac{P_{d}}{P_{\text {free_field }}}=\frac{e^{-i \pi / 4}}{\sqrt{\pi}}\left[\frac{R}{L} F\left(X_{-}\right)+\frac{R}{L} e^{i k(S-R)} F\left(X_{+}\right)\right]
$$

$$
N_{1}=2 \frac{L-R}{\lambda}, N_{2}=2 \frac{L-S}{\lambda}, k=\frac{2 \pi}{\lambda}
$$

$N_{1}$ is the Fresnel number for the real source, $N_{2}$ is the Fresnel number for the image source. Therefore, De Jong's model based on MacDonald solution can be expressed in terms of Fresnel Number $N_{1}$ and $N_{2}$ in Equation (7-7):

$$
\frac{P_{d}}{P_{\text {free_field }}}=\frac{e^{-i \pi / 4}}{\sqrt{\pi}} \frac{R}{L}\left[F\left(\sqrt{\pi N_{1}}\right)+e^{i k(S-R)} F\left(\sqrt{\pi N_{2}}\right)\right]
$$

According to the TNM Technical Manual, the diffraction term is defined in Equation (7-8):

$$
D=\frac{R}{L} \frac{e^{-i \frac{\pi}{4}}}{\sqrt{\pi}} e^{i k(L-R)} e^{-i \chi^{2}} F(\chi)
$$


Comparing Equation (7-8) with Equation (7-1), we can see that, TNM just deployed MacDonald solution with the real source contribution only. TNM does not take into account the image source contribution. It was pointed out that the contribution from image source in the barrier should not be overlooked (T Isei, 1980). In the following section, we will discuss how this modification influences the insertion loss. In addition, Kurze-Anderson's formula will be compared at the same time, since it is deployed in the STAMINA model for the diffraction.

Figure 7-20 and Figure 7-21 are two plots for the insertion loss comparison based on different geometry configurations. The $\mathrm{x}$-axis represents Fresnel Number from 0.01 to 10. The $y$-axis stands for insertion loss in dBA. For general highway barrier geometry settings, the Fresnel Number is often greater than 0.1. The curve for MacDonald's solution with the real source and the image source is below that of MacDonald's solution without image source, and above that of Maekawa's chart.

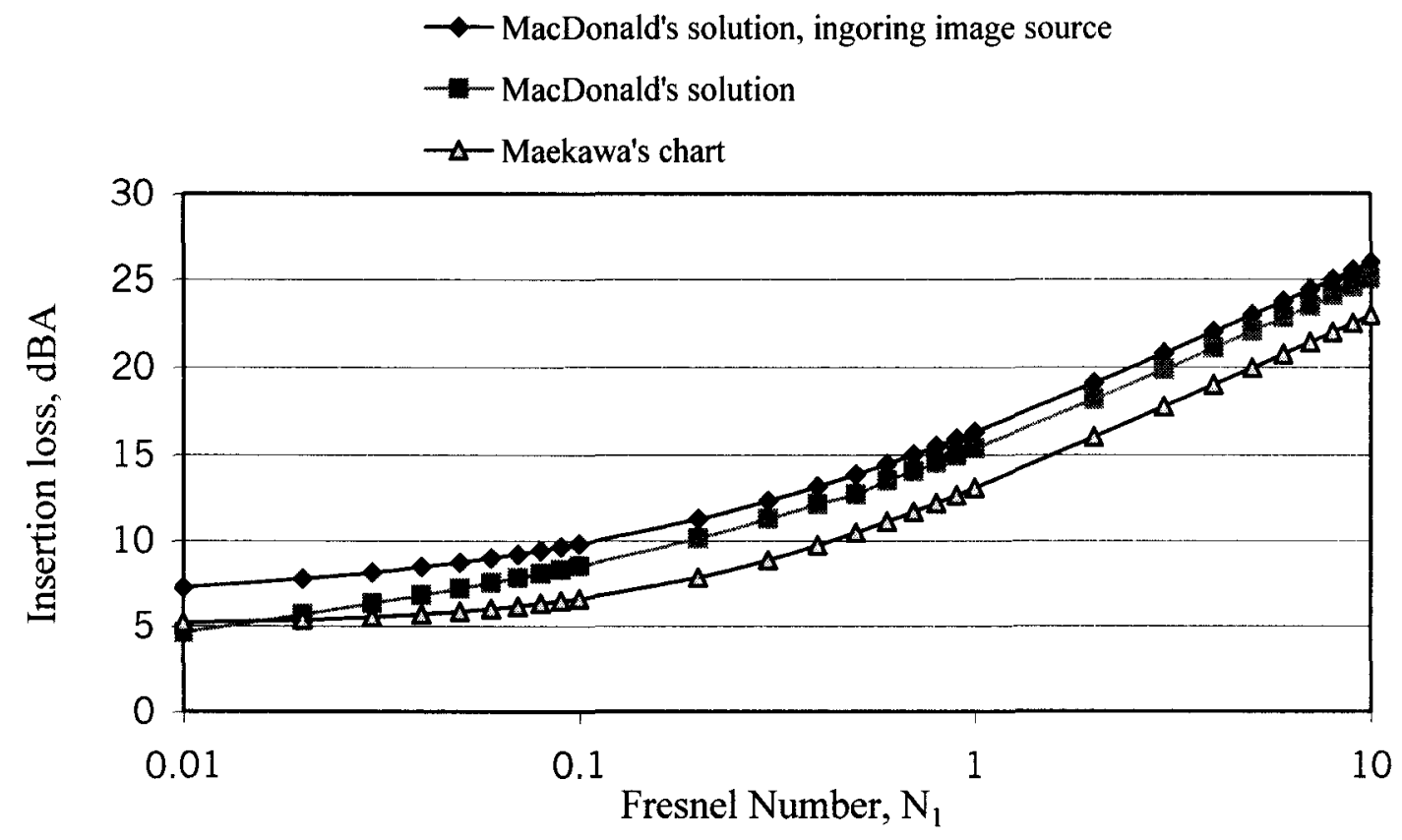

Figure 7-20. Comparison of Maekawa's chart and MacDonald's solution, with $\mathrm{N}_{2}: \mathrm{N}_{\mathrm{I}}=$ $80: 1$ 


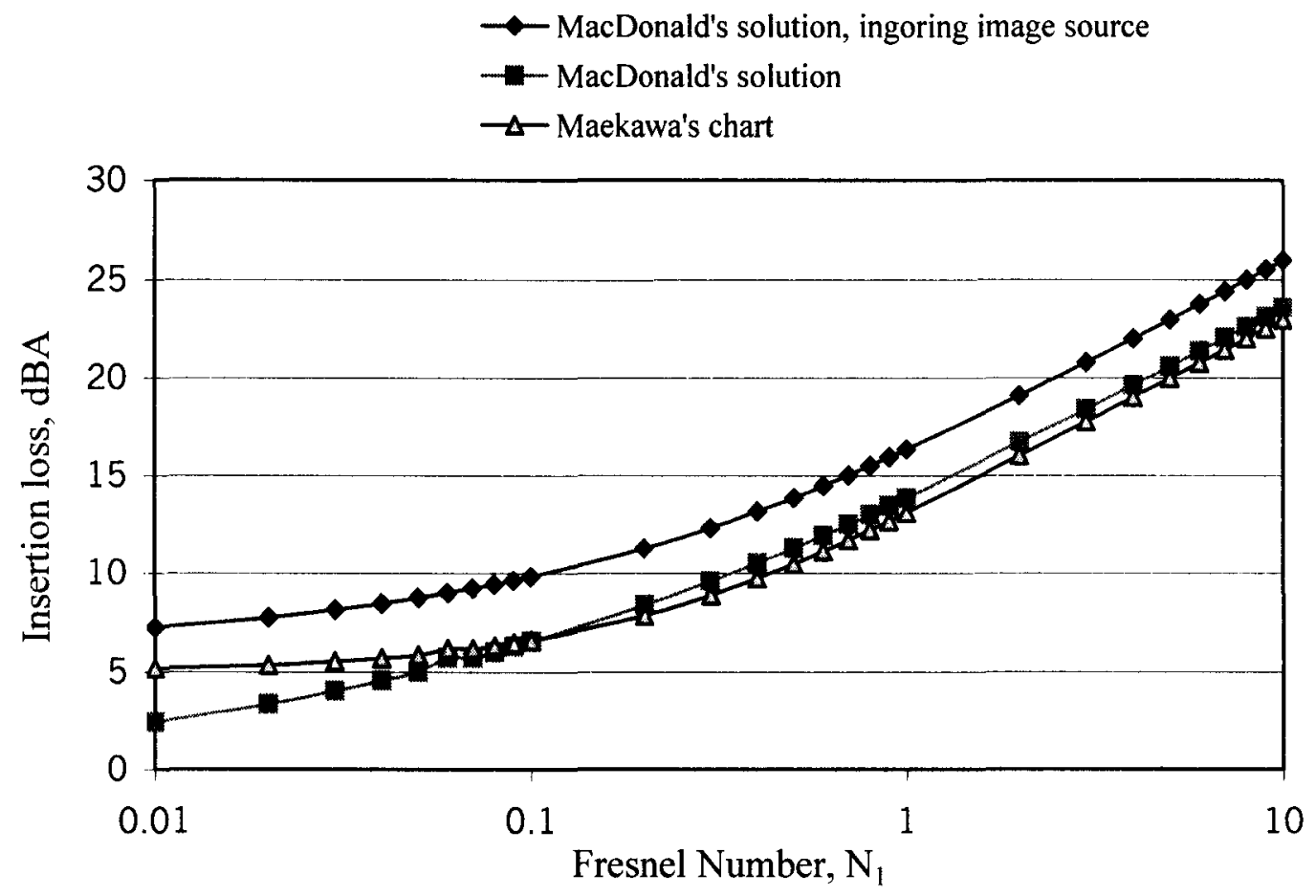

Figure 7-21. Comparison of Maekawa's chart and MacDonald's solution, with $\mathrm{N}_{2}: \mathrm{N}_{1}=$ $10: 1$
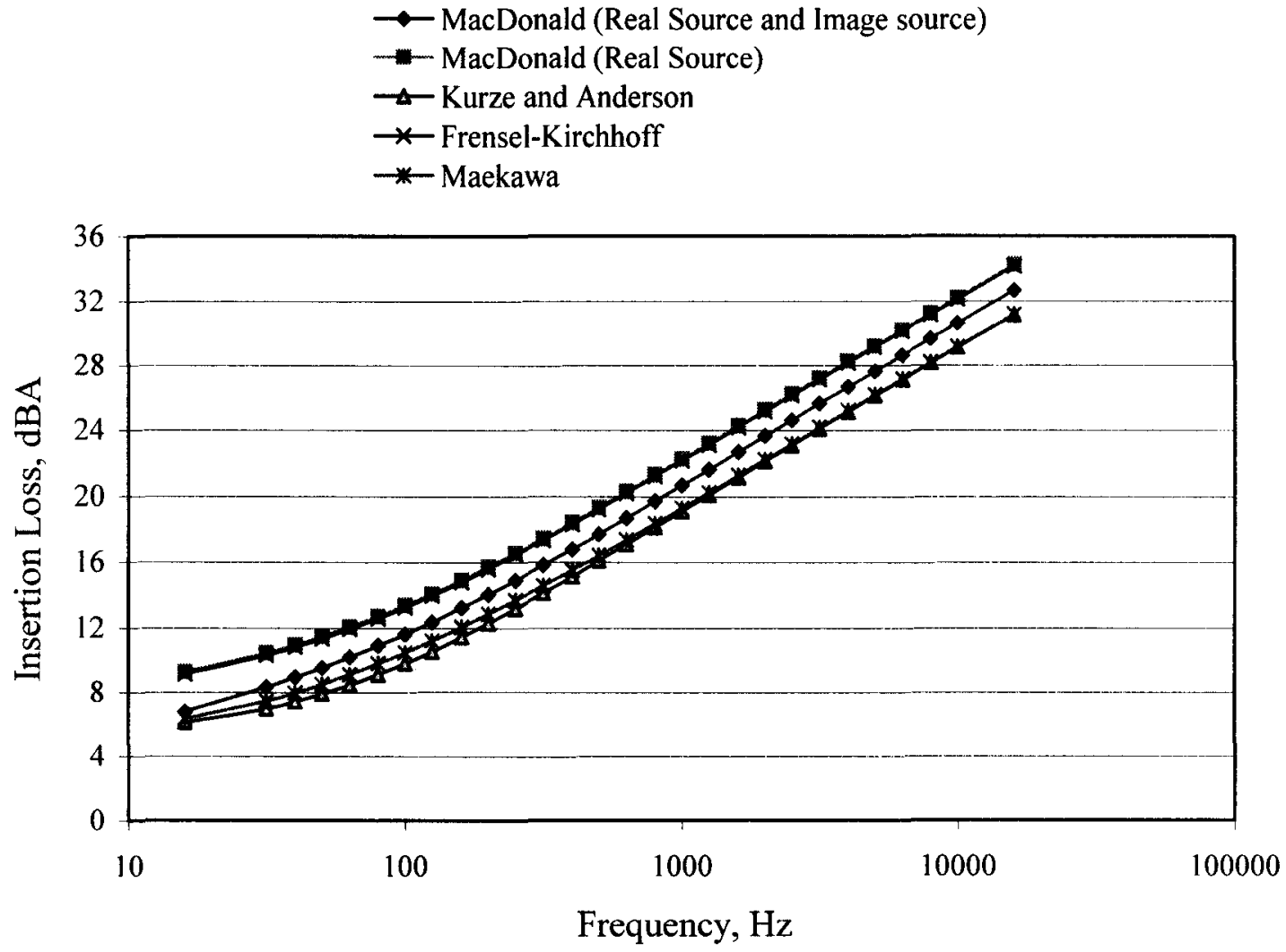

Figure 7-22. Insertion loss comparison by different diffraction theories 
By transforming the $\mathrm{x}$-axis from Fresnel Number to frequency, the difference of insertion loss by diffraction curves can be observed in frequency domain. Five curves representing different diffraction theories are illustrated in Figure 7-22, mainly the complete MacDonald's solution with real and image source contributions, MacDonald's solution without image source, Kurze-Anderson's curve, Fresnel-Kirchhoff's theory and Maekawa's curve. When ignoring image source contribution, MacDonald solution is very similar to the Fresnel-Kirchhoff theory, since Kawai (1978) stated that FresnelKirchhoff's theory does not consider the image source contribution either, which results in insertion loss over-predictions (Maekawa, 1968). Therefore, MacDonald's solution without image source contribution also has the tendency of over-predicting the insert ion loss. Kurze-Anderson's curve is developed based on the Maekawa's measurements. The deviation between these two curves is within $1.0 \mathrm{dBA}$ for the low frequency bands in this case. The insertion loss of MacDonald's solution is greater than that of Maekawa's curve and less than that of MacDonald's solution without image source contribution.

In the following part, the differences of insertion loss will be examined in detail based on ten cases. At this point, we focus on MacDonald's solution and KurzeAnderson's theory, because they are the diffraction theories used in TNM, STAMINA and HNP.

Case 1 to case 5 will examine the varying distances of the source from the barrier. Other parameter related to the geometrical configuration is as follows: source height 1.5 $\mathrm{m}(5 \mathrm{ft})$, barrier height $5 \mathrm{~m}(16 \mathrm{ft})$, receiver to barrier distance $15 \mathrm{~m}(50 \mathrm{ft})$, and receiver height $1.5 \mathrm{~m}(5 \mathrm{ft})$. 


\section{Case 1:}

In this case, source to barrier distance is $5 \mathrm{~m}(16 \mathrm{ft})$. Figure 7-23 shows the plot of insertion loss for the three models and their differences are shown in Table 7-6 for the given configurations.

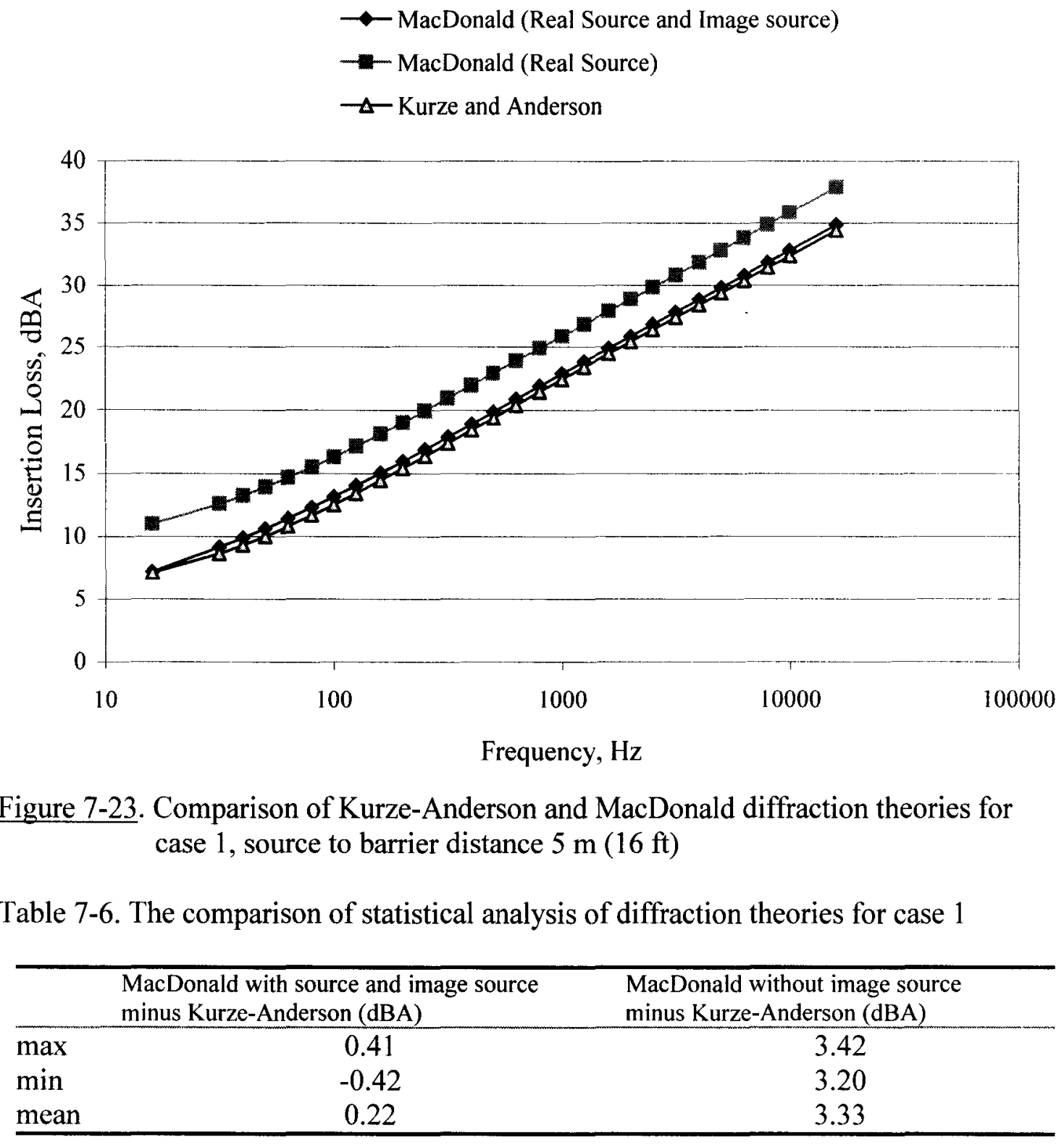


Case 2:

In this case, source to barrier distance is $20 \mathrm{~m}(66 \mathrm{ft})$. Figure 7-24 shows the plot of insertion loss for the three models and their differences are shown in Table 7-7 for the given configurations.

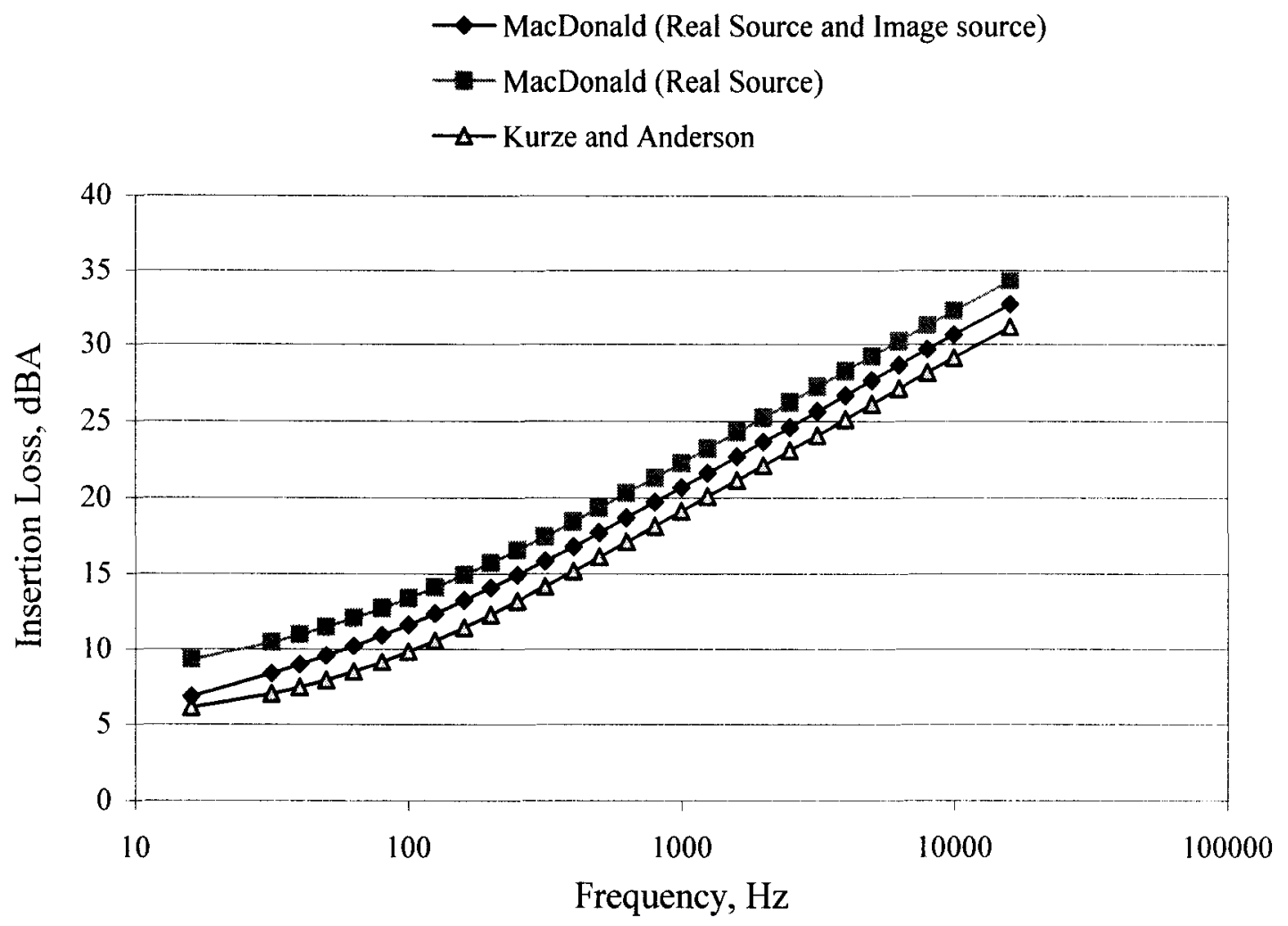

Figure 7-24. Comparison of Kurze-Anderson and MacDonald diffraction theory for case 2 , source to barrier distance $20 \mathrm{~m}(66 \mathrm{ft})$

Table 7-7. The comparison of statistical analysis of diffraction theories for case 2

\begin{tabular}{lcc}
\hline & $\begin{array}{l}\text { MacDonald with source and image source } \\
\text { minus Kurze-Anderson (dBA) }\end{array}$ & $\begin{array}{l}\text { MacDonald without image source } \\
\text { minus Kurze-Anderson (dBA) }\end{array}$ \\
\hline $\max$ & 1.49 & 3.07 \\
$\min$ & 0.46 & 2.84 \\
mean & 1.26 & 2.96 \\
\hline
\end{tabular}




\section{Case 3:}

In this case, source to barrier distance is $35 \mathrm{~m}(115 \mathrm{ft})$. Figure 7-25 shows the plot of insertion loss for the three models and their differences are shown in Table 7-8 for the given configurations.

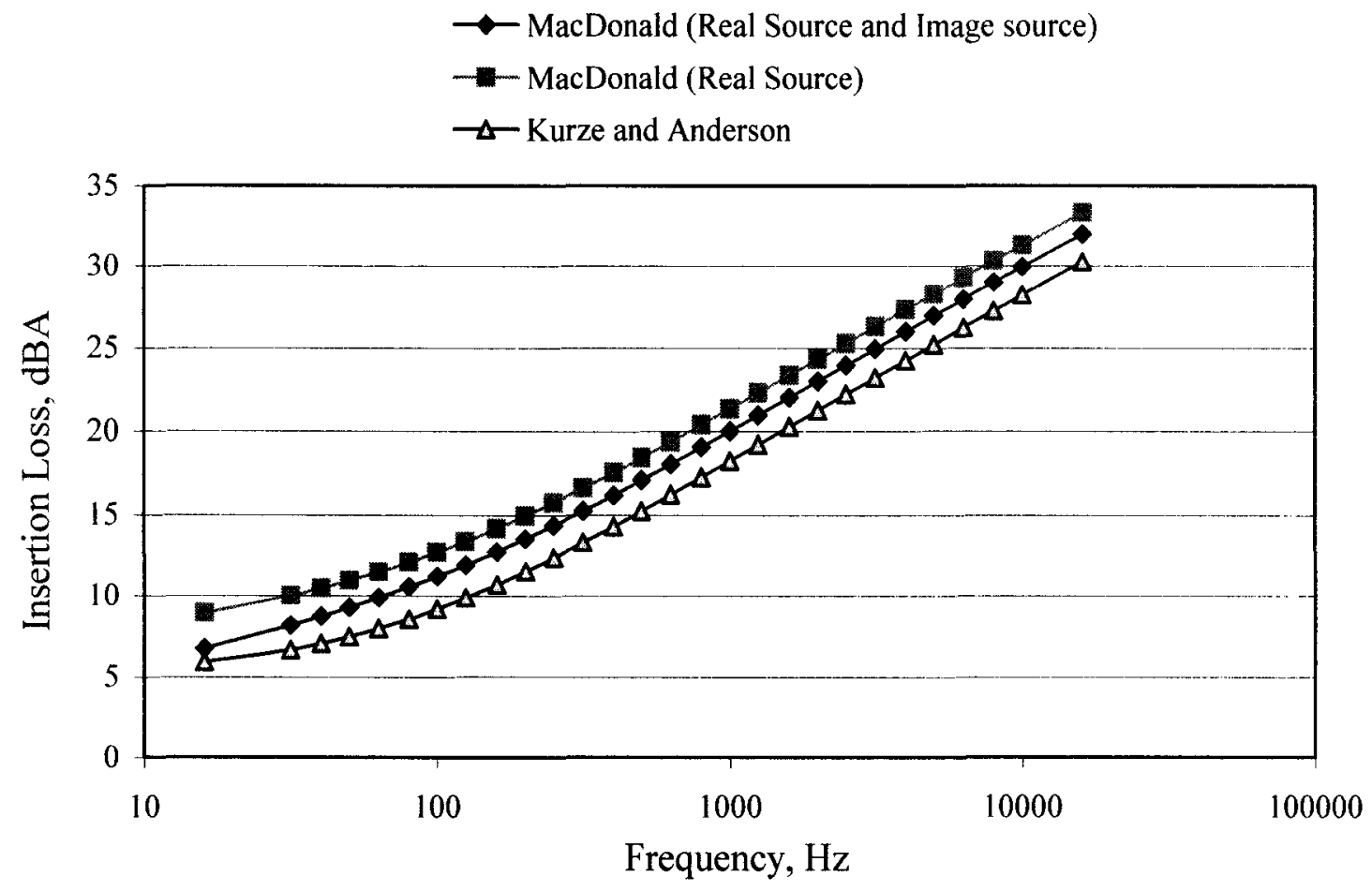

Figure 7-25. Comparison of Kurze-Anderson and MacDonald diffraction theory for case 3 , source to barrier distance $35 \mathrm{~m}(115 \mathrm{ft})$

Table 7-8. The comparison of statistical analysis of diffraction theories for case 3

\begin{tabular}{lcc}
\hline & $\begin{array}{l}\text { MacDonald with source and image source } \\
\text { minus Kurze-Anderson (dBA) }\end{array}$ & $\begin{array}{l}\text { MacDonald without image source } \\
\text { minus Kurze-Anderson (dBA) }\end{array}$ \\
\hline $\max$ & 1.70 & 3.02 \\
$\min$ & 0.64 & 2.78 \\
mean & 1.46 & 2.90 \\
\hline
\end{tabular}


Case 4:

In this case, source to barrier distance is $50 \mathrm{~m}(164 \mathrm{ft})$. Figure 7-26 shows the plot of insertion loss for the three models and their differences are shown in Table 7-9 for the given configurations.

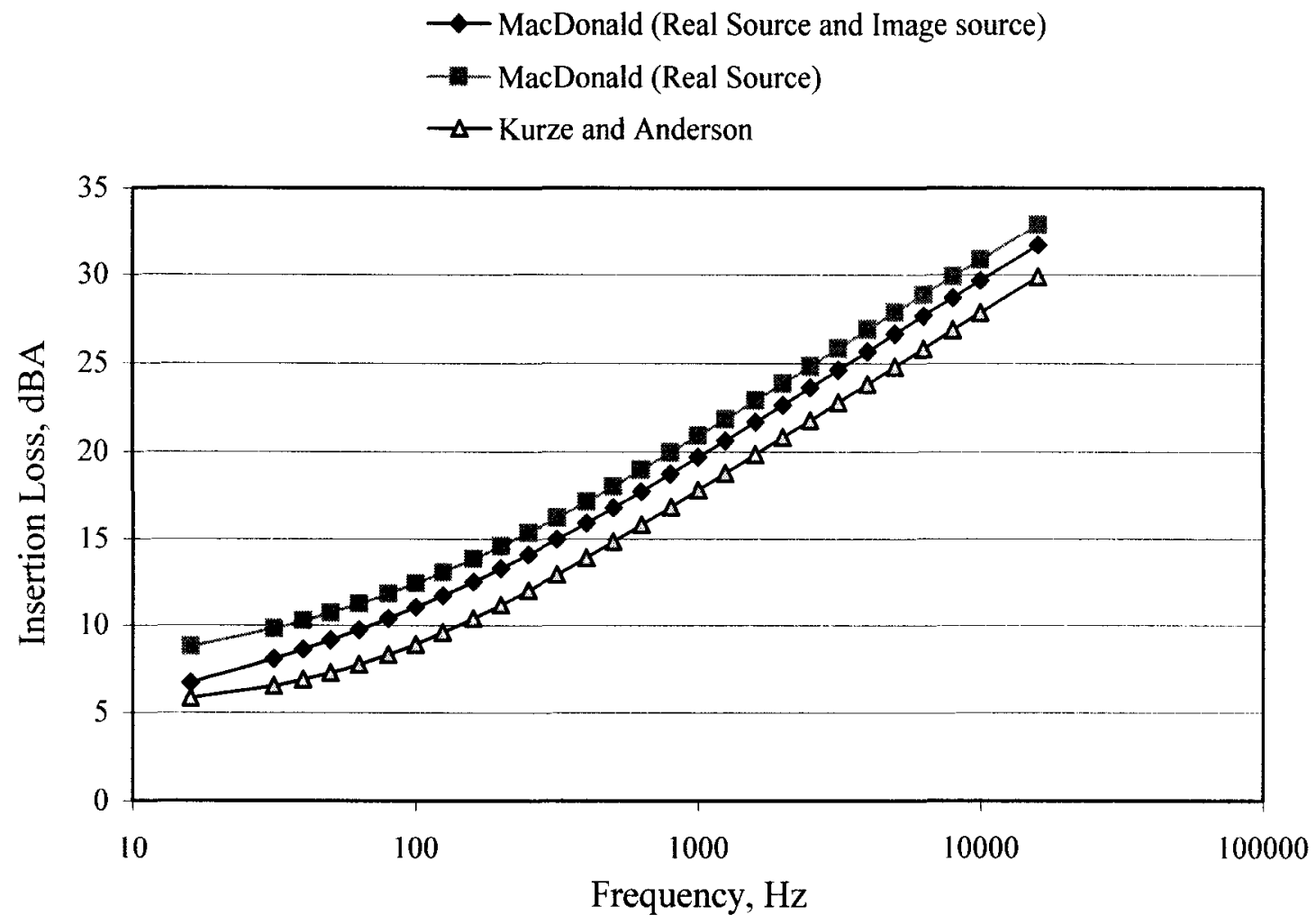

Figure 7-26. Comparison of Kurze-Anderson and MacDonald diffraction theory for case 4 , source to barrier distance $50 \mathrm{~m}(164 \mathrm{ft})$

Table 7-9. The comparison of statistical analysis of diffraction theories for case 4

\begin{tabular}{lcc}
\hline & $\begin{array}{c}\text { MacDonald with source and image source } \\
\text { minus Kurze-Anderson (dBA) }\end{array}$ & $\begin{array}{l}\text { MacDonald without image source } \\
\text { minus Kurze-Anderson (dBA) }\end{array}$ \\
\hline $\max$ & 1.78 & 2.99 \\
$\min$ & 0.71 & 2.76 \\
mean & 1.54 & 2.87 \\
\hline
\end{tabular}




\section{Case 5:}

In this case, source to barrier distance is $65 \mathrm{~m}(213 \mathrm{ft})$. Figure 7-27 shows the plot of insertion loss for the three models and their differences are shown in Table 7-10 for the given configurations.

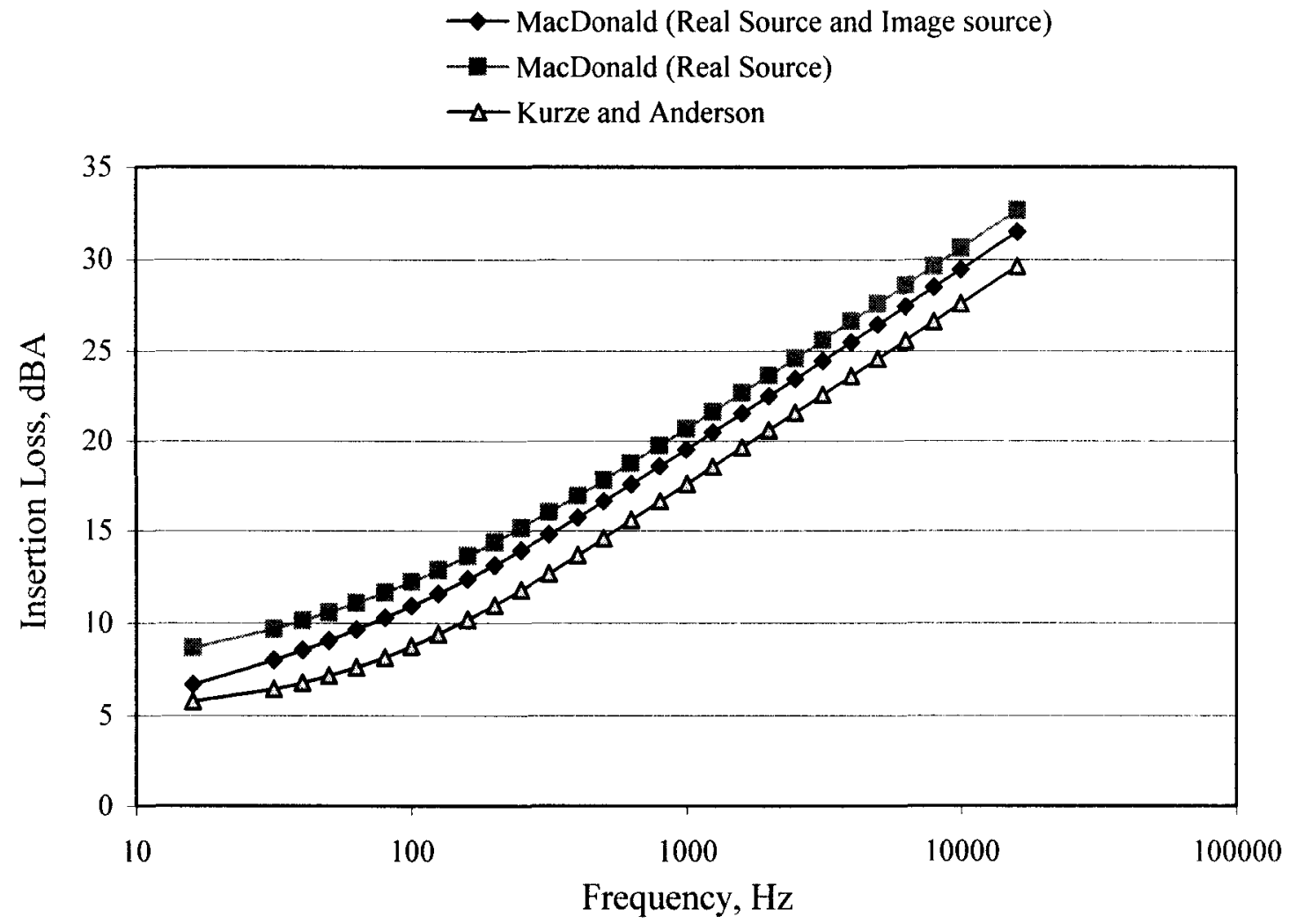

Figure 7-27. Comparison of Kurze-Anderson and MacDonald diffraction theory for case 5 , source to barrier distance $65 \mathrm{~m}(213 \mathrm{ft})$

Table 7-10. The comparison of statistical analysis of diffraction theories for case 5

\begin{tabular}{lcc}
\hline & $\begin{array}{l}\text { MacDonald with source and image source } \\
\text { minus Kurze-Anderson (dBA) }\end{array}$ & $\begin{array}{l}\text { MacDonald without image source } \\
\text { minus Kurze-Anderson (dBA) }\end{array}$ \\
\hline max & 1.83 & 2.98 \\
min & 0.75 & 2.74 \\
mean & 1.59 & 2.85 \\
\hline
\end{tabular}


Figure 7-28 represents the difference of insertion loss by MacDonald and KurzeAnderson curve with varying source distances. Based on case 1 to case 5, the following findings can be made: When the source is close to the barrier, the curve of MacDonald's solution is close to that of Maekawa's theory, with an error of $0.2 \mathrm{dBA}$ in all frequency bands. When the distance from the source to the barrier becomes greater, the curve of MacDonald's solution is moving towards the curve of MacDonald's solution without image source. However, their difference is about $2 \mathrm{dBA}$ when the source distance from the barrier is greater than $20 \mathrm{~m}(66 \mathrm{ft})$. Based on this finding, we can conclude that from the perspective of diffraction theory, the average difference for insertion loss of TNM and STAMINA is about $3 \mathrm{dBA}$. If MacDonald's solution with source and image source is applied in TNM, the difference of insertion loss tends to be smaller, especially when the source is close to the barrier, compared with STAMINA.

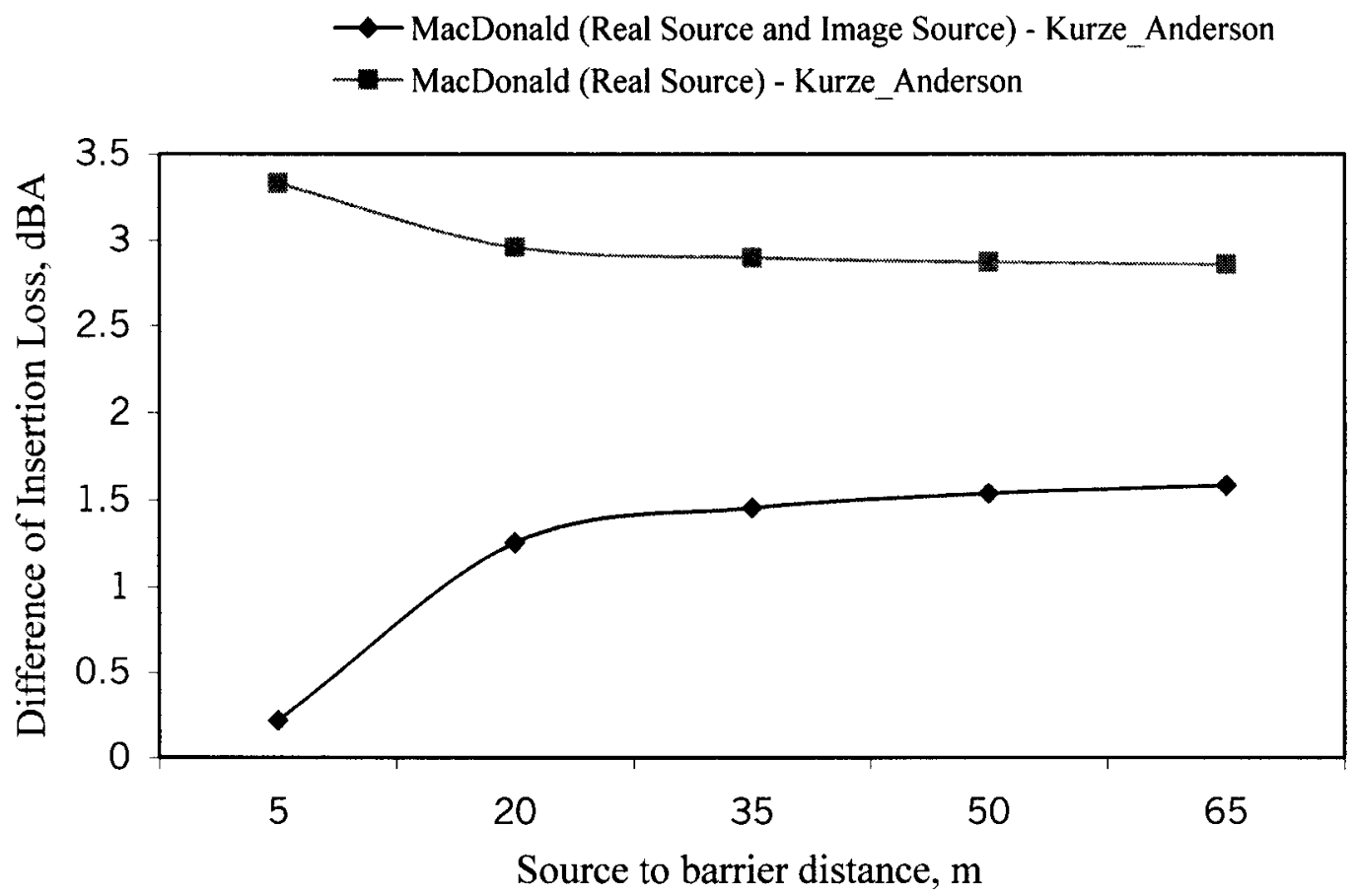

Figure 7-28. Difference of insertion loss by MacDonald's curve and Kurze-Anderson's curve with varying source distances 
Case 6 to case 10 will examine the varying distances of the receiver from the barrier. Other parameter related to the geometrical configuration is as follows: source to barrier distance15 $\mathrm{m}(50 \mathrm{ft})$, source height $1.5 \mathrm{~m}(5 \mathrm{ft})$, barrier height $5 \mathrm{~m}(16 \mathrm{ft})$, and receiver height $1.5 \mathrm{~m}(5 \mathrm{ft})$.

Case 6:

In this case, receiver to barrier distance is $5 \mathrm{~m}(16 \mathrm{ft})$. Figure 7-29 shows the plot of insertion loss for the three models and their differences are shown in Table 7-11 for the given configurations.
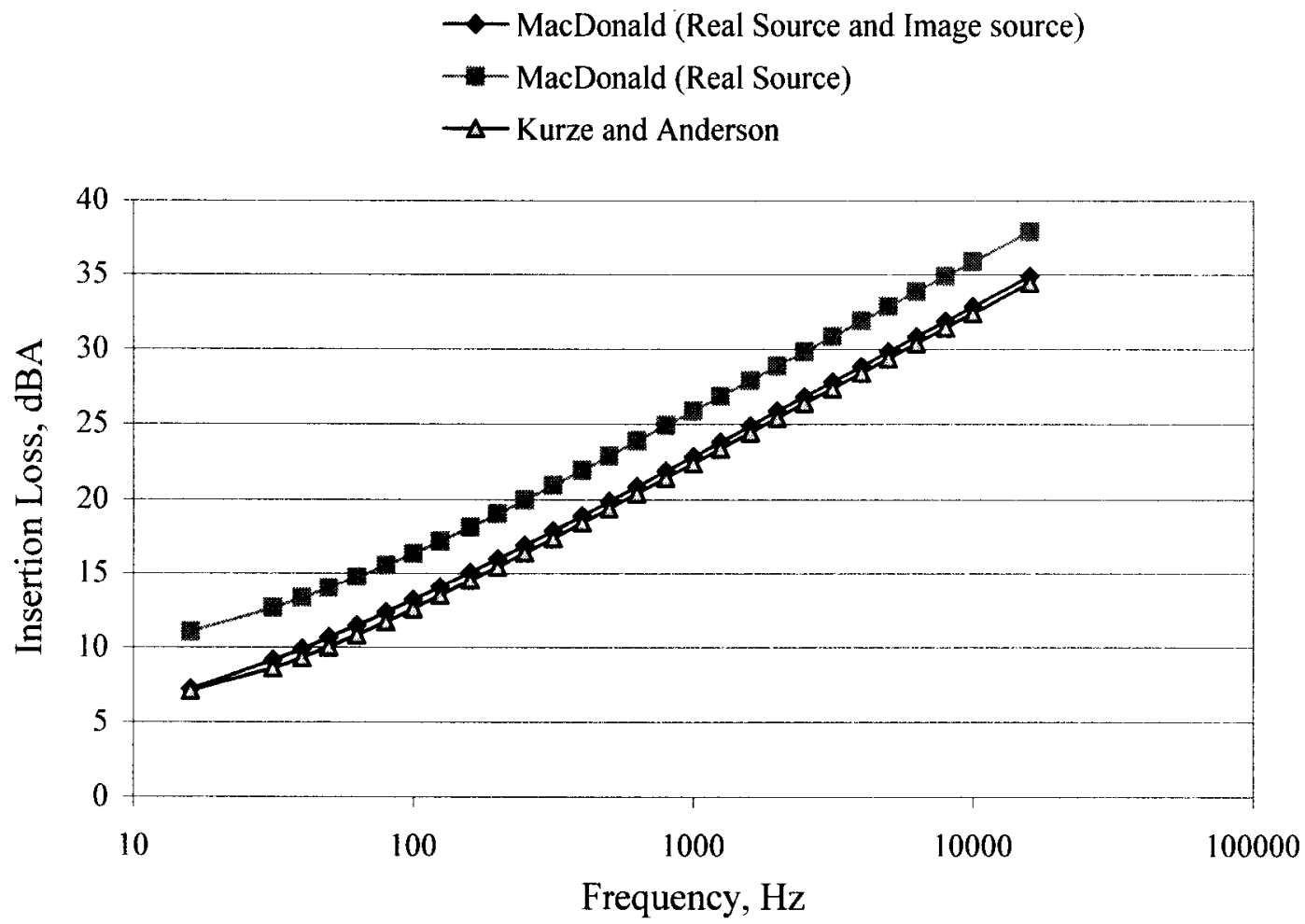

Figure 7-29. Comparison of Kurze-Anderson and MacDonald diffraction theory for case 6 , receiver to barrier distance $5 \mathrm{~m}(16 \mathrm{ft})$ 
Table 7-11. The comparison of statistical analysis of diffraction theories for case 6

\begin{tabular}{lcc}
\hline & $\begin{array}{l}\text { MacDonald with source and image source } \\
\text { minus Kurze-Anderson (dBA) }\end{array}$ & $\begin{array}{l}\text { MacDonald without image source } \\
\text { minus Kurze-Anderson (dBA) }\end{array}$ \\
\hline $\max$ & 0.41 & 3.42 \\
$\min$ & -0.42 & 3.20 \\
mean & 0.22 & 3.33 \\
\hline
\end{tabular}

\section{Case 7:}

In this case, receiver to barrier distance is $20 \mathrm{~m}(66 \mathrm{ft})$. Figure $7-30$ shows the plot of insertion loss for the three models and their differences are shown in Table 7-12 for the given configurations.
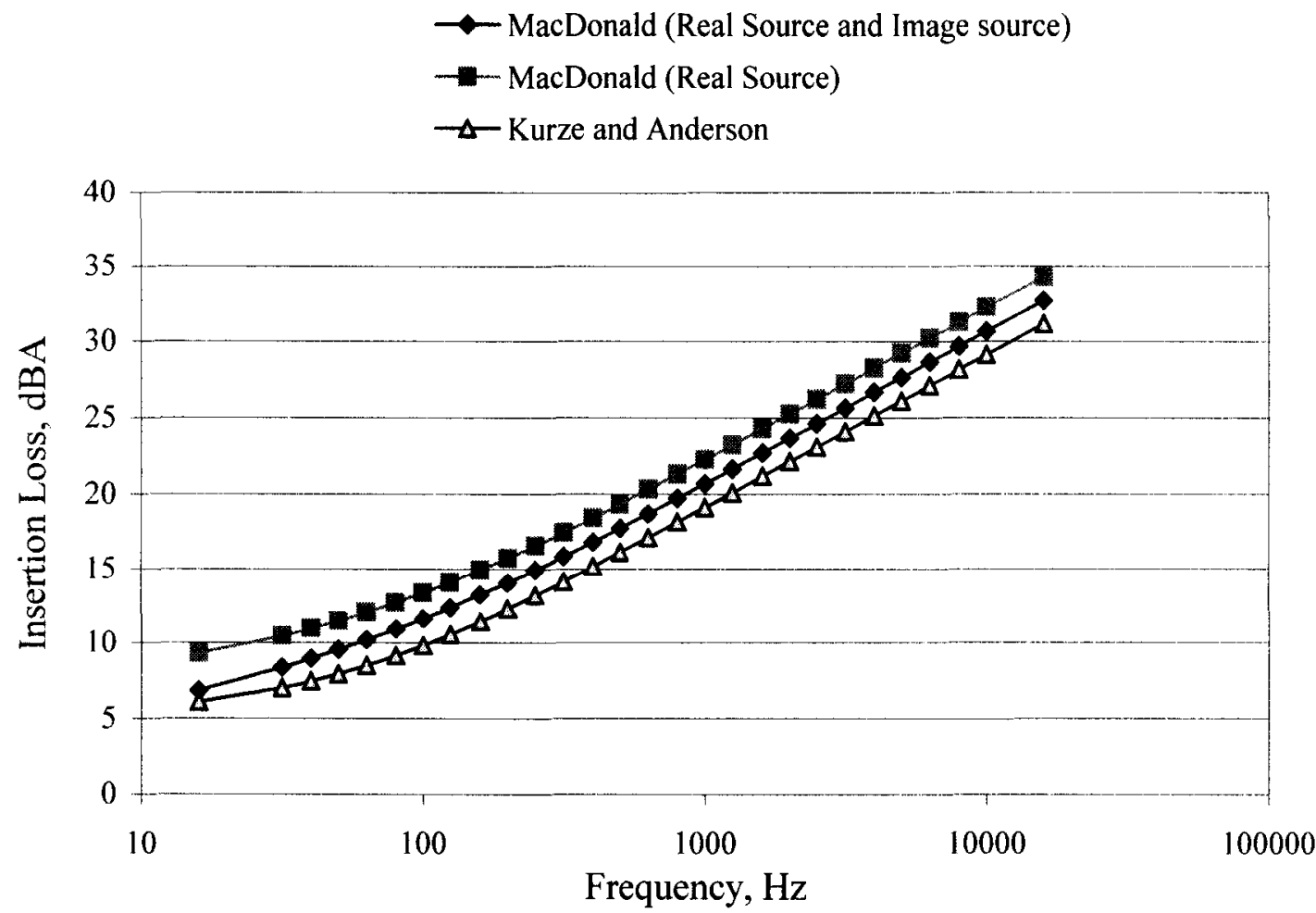

Figure 7-30. Comparison of Kurze-Anderson and MacDonald diffraction theory for case 7 , receiver to barrier distance $20 \mathrm{~m}(66 \mathrm{ft})$ 
Table 7-12. The comparison of statistical analysis of diffraction theories for case 7

\begin{tabular}{lcc}
\hline & $\begin{array}{l}\text { MacDonald with source and image source } \\
\text { minus Kurze-Anderson (dBA) }\end{array}$ & $\begin{array}{l}\text { MacDonald without image source } \\
\text { minus Kurze-Anderson (dBA) }\end{array}$ \\
\hline $\max$ & 1.49 & 3.07 \\
$\min$ & 0.46 & 2.84 \\
mean & 1.26 & 2.96 \\
\hline
\end{tabular}

Case 8:

In this case, receiver to barrier distance is $35 \mathrm{~m}(115 \mathrm{ft})$. Figure 7-31 shows the plot of insertion loss for the three models and their differences are shown in Table 7-13 for the given configurations.

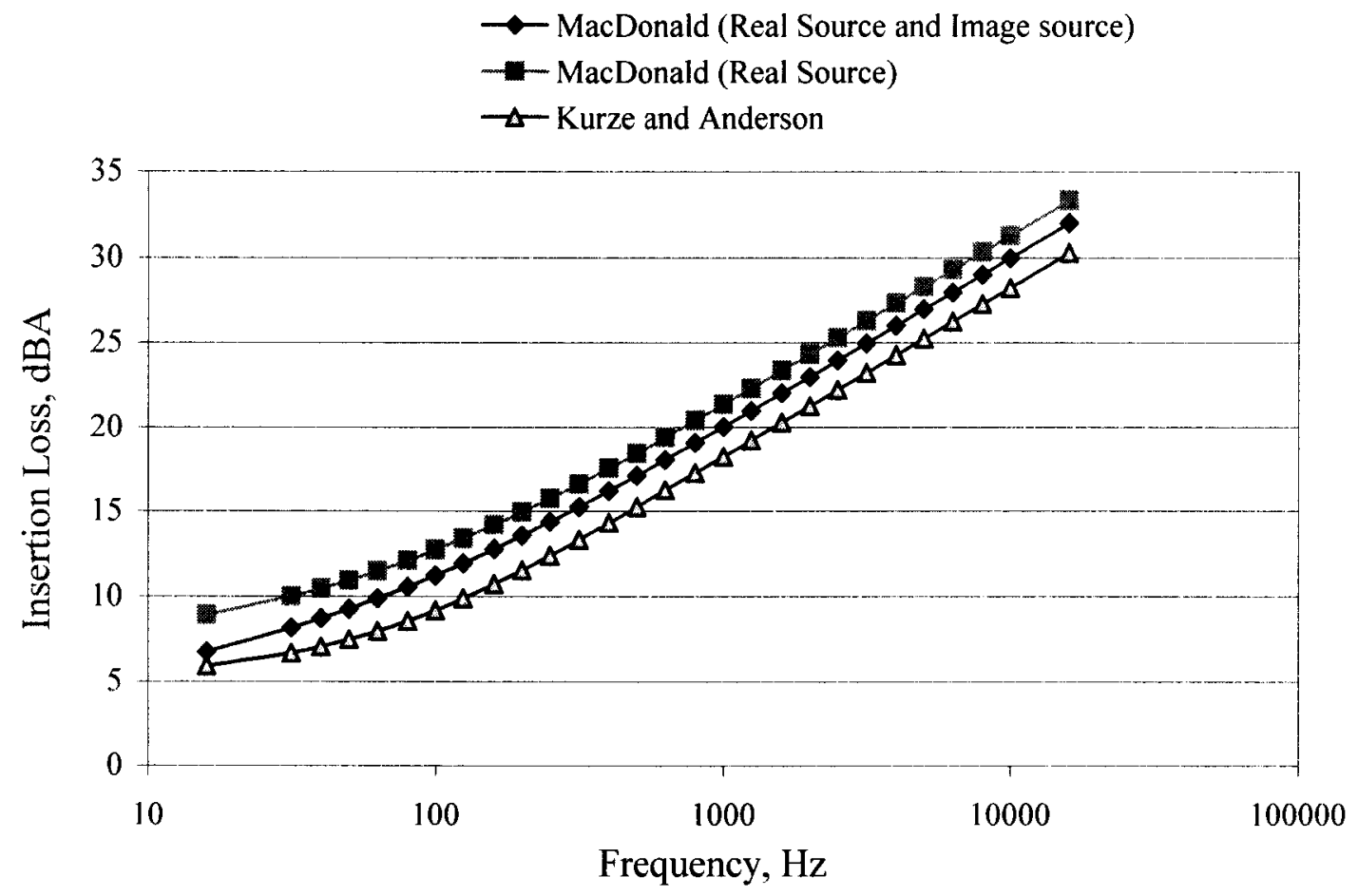

Figure 7-31. Comparison of Kurze-Anderson and MacDonald diffraction theory for case 8 , receiver to barrier distance $35 \mathrm{~m}(115 \mathrm{ft})$ 
Table 7-13. The comparison of statistical analysis of diffraction theories for case 8

\begin{tabular}{lcc}
\hline & $\begin{array}{l}\text { MacDonald with source and image source } \\
\text { minus Kurze-Anderson (dBA) }\end{array}$ & $\begin{array}{l}\text { MacDonald without image source } \\
\text { minus Kurze-Anderson (dBA) }\end{array}$ \\
\hline $\max$ & 1.70 & 3.02 \\
$\min$ & 0.64 & 2.78 \\
mean & 1.46 & 2.90 \\
\hline
\end{tabular}

Case 9:

In this case, receiver to barrier distance is $50 \mathrm{~m}(164 \mathrm{ft})$. Figure 7-32 shows the plot of insertion loss for the three models and their differences are shown in Table 7-14 for the given configurations.

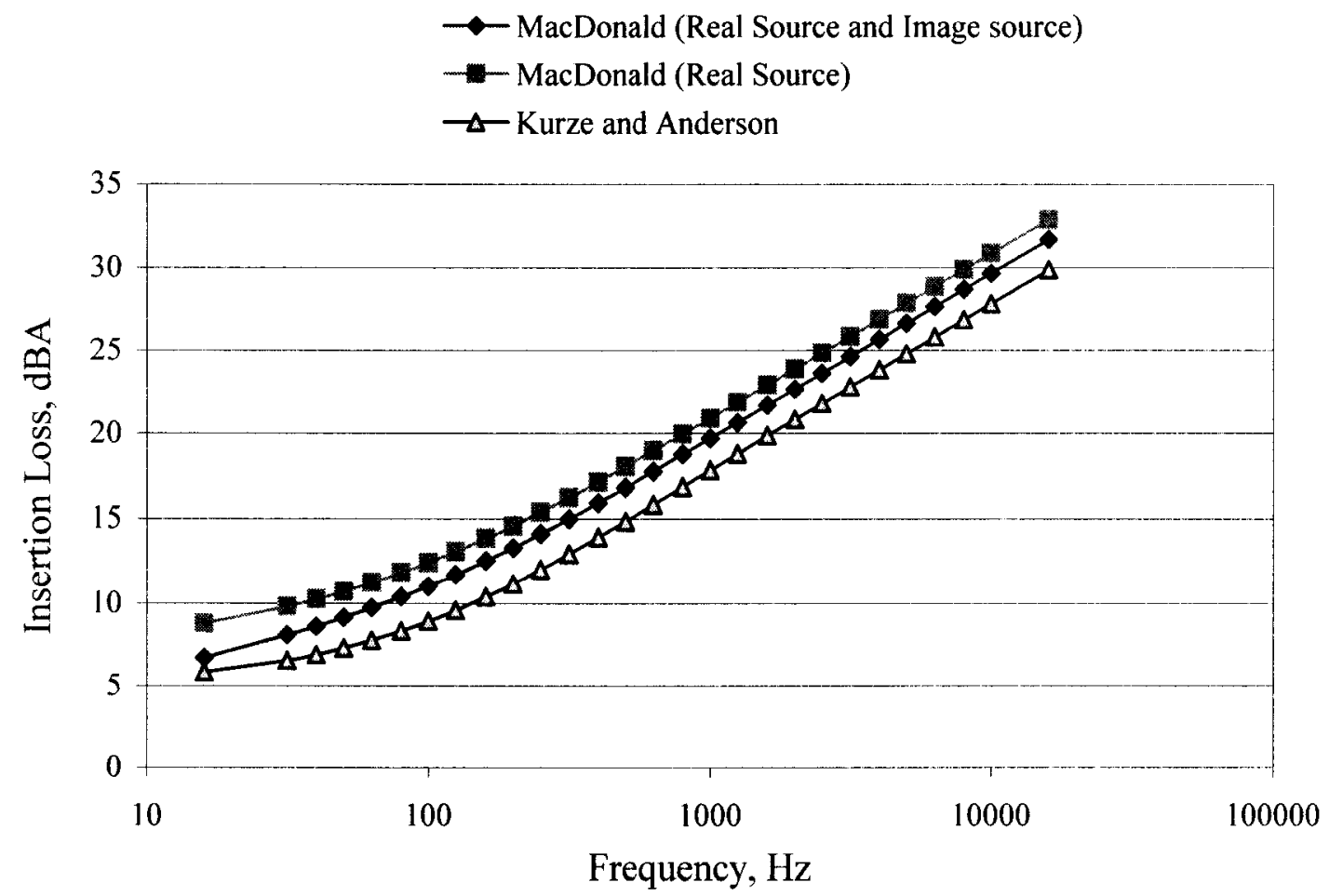

Figure 7-32. Comparison of Kurze-Anderson and MacDonald diffraction theory for case 9 , receiver to barrier distance $50 \mathrm{~m}(164 \mathrm{ft})$ 
Table 7-14. The comparison of statistical analysis of diffraction theories for case 9

\begin{tabular}{lcc}
\hline & $\begin{array}{l}\text { MacDonald with source and image source } \\
\text { minus Kurze-Anderson (dBA) }\end{array}$ & $\begin{array}{l}\text { MacDonald without image source } \\
\text { minus Kurze-Anderson (dBA) }\end{array}$ \\
\hline $\max$ & 1.78 & 2.99 \\
$\min$ & 0.71 & 2.76 \\
mean & 1.54 & 2.87 \\
\hline
\end{tabular}

Case 10:

In this case, receiver to barrier distance is $65 \mathrm{~m}(213 \mathrm{ft})$. Figure 7-33 shows the plot of insertion loss for the three models and their differences are shown in Table 7-15 for the given configurations.

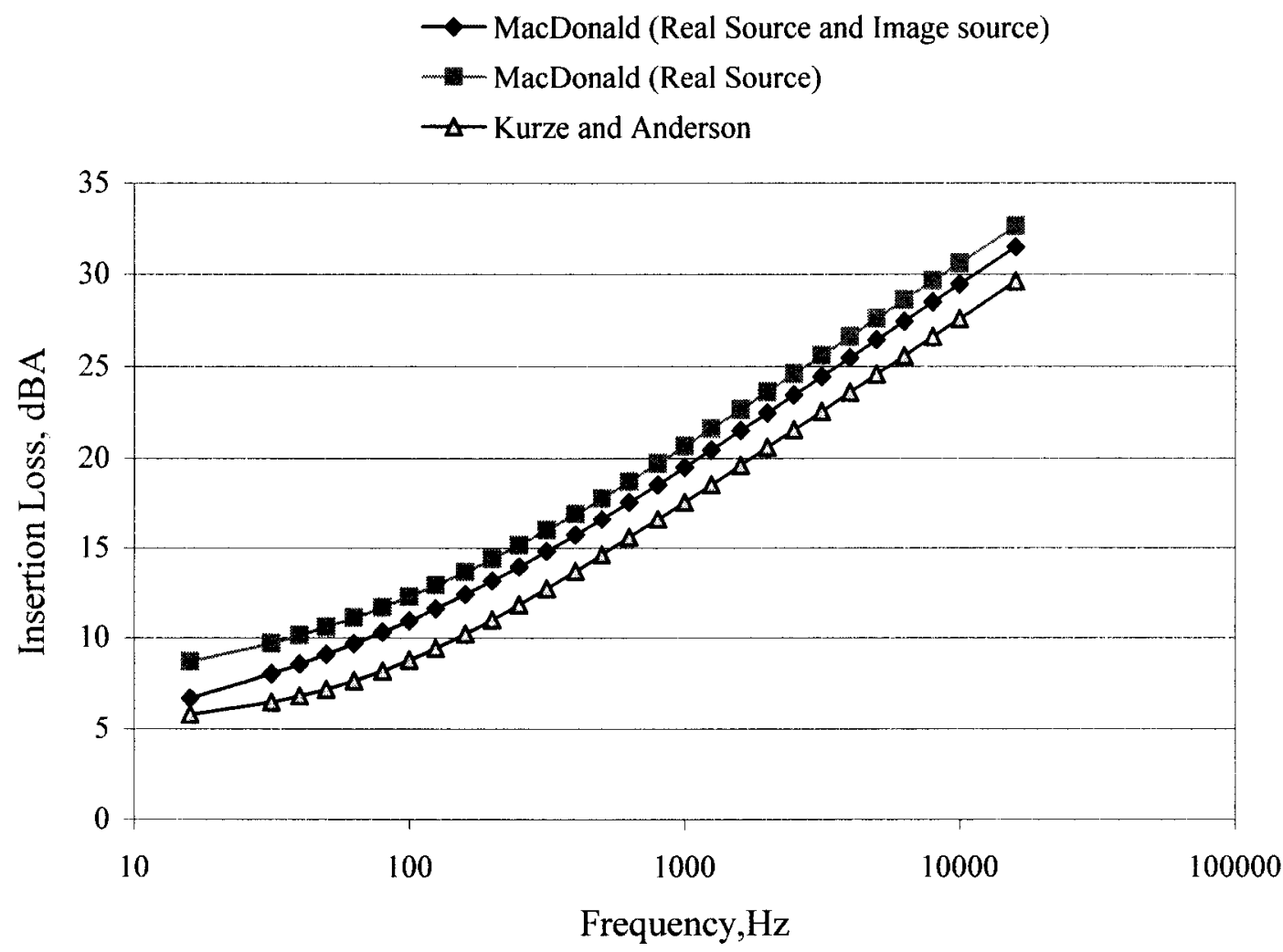

Figure 7-33. Comparison of Kurze-Anderson and MacDonald diffraction theory for case 10 , receiver to barrier distance $65 \mathrm{~m} \mathrm{(213 \textrm {ft } )}$ 
Table 7-15. The comparison of statistical analysis of diffraction theories for case 10

\begin{tabular}{lcc}
\hline & $\begin{array}{l}\text { MacDonald with source and image source } \\
\text { minus Kurze-Anderson (dBA) }\end{array}$ & $\begin{array}{l}\text { MacDonald without image source } \\
\text { minus Kurze-Anderson (dBA) }\end{array}$ \\
\hline $\max$ & 1.83 & 2.98 \\
$\min$ & 0.75 & 2.74 \\
mean & 1.59 & 2.85 \\
\hline
\end{tabular}

Figure 7-34 suggests the following findings based on case 6 to case 10 . When the receiver is close to the barrier, the curve for the MacDonald's solution is close to Maekawa's curve, with an average difference of $0.2 \mathrm{dBA}$ in all frequency bands. When the distance from the receiver to the barrier becomes greater, the curve for the MacDonald's solution is moving towards the curve of MacDonald's solution without image source. However, their difference is still about $2 \mathrm{dBA}$ when the receiver distance from the barrier is larger than $20 \mathrm{~m}$. Conclusion can be made that from the perspective of diffraction theory, the average difference of insertion loss of TNM and STAMINA is about $3 \mathrm{dBA}$. If MacDonald's solution with source and image source is deployed in TNM, the difference of insertion loss tends to be smaller, especially when the receiver is close to the barrier. 


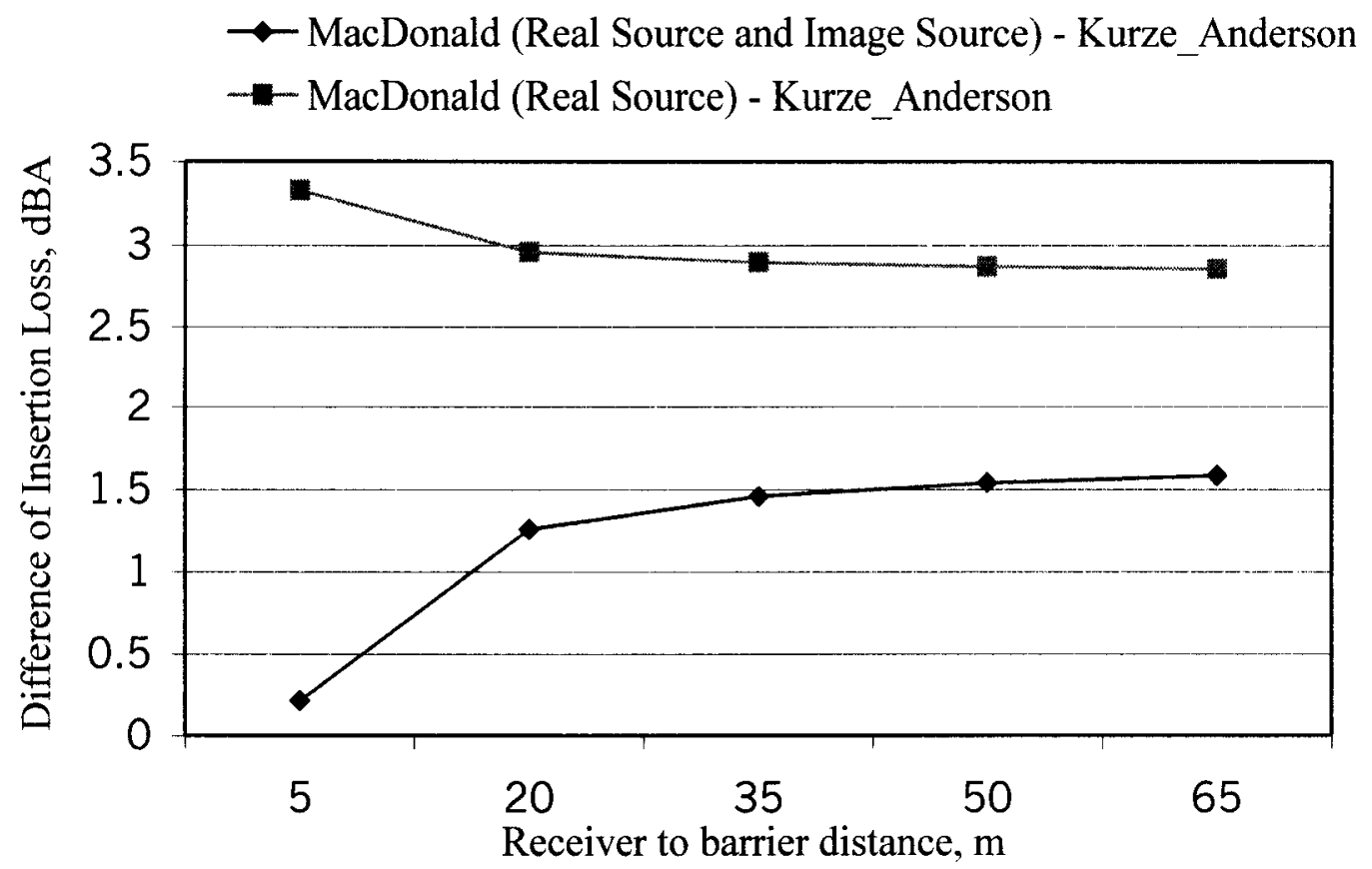

Figure 7-34. Difference of insertion loss by MacDonald and Kurze-Anderson curve with varying receiver distances

\subsection{Effect on Insertion Loss by Reduction from Four Propagation Paths to Two Propagation Paths}

According to Menge (1996) and the TNM Technical Manual (1998), TNM does not take into account the noise reflection on the receiver side behind the barrier. For a common geometrical configuration with a source point and a receiver point on both sides of a single noise barrier, only two propagation paths are considered in TNM, shown as in Figure 7-35. 


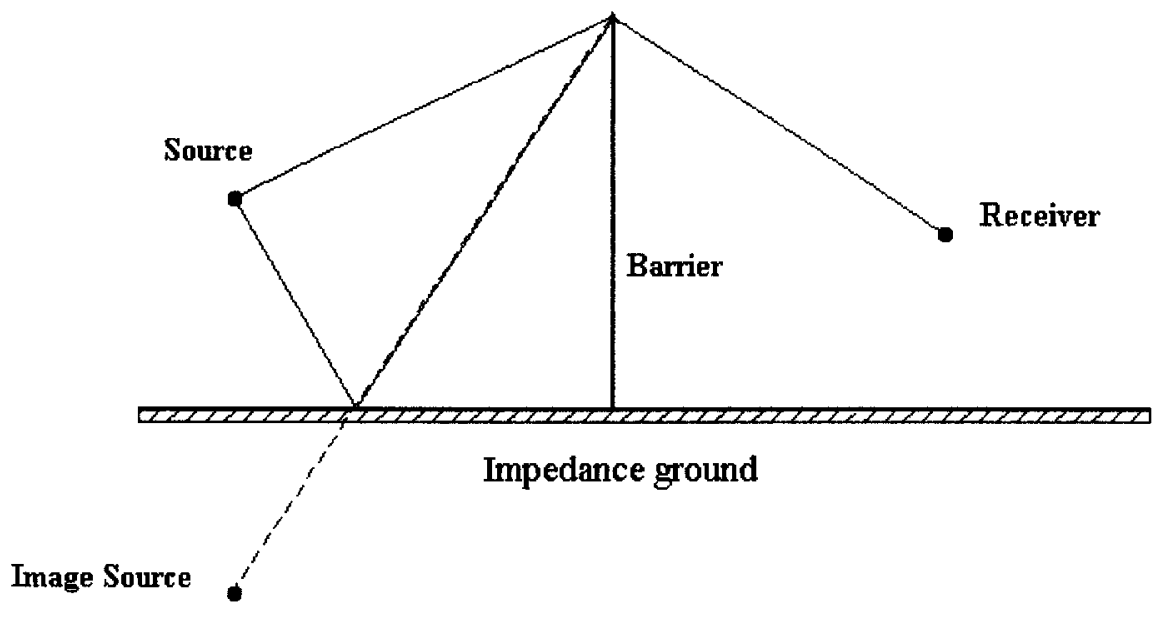

Figure 7-35. Two propagation paths in TNM

TNM computes the sound levels based on the complex manner within all the frequency bands. In some specific frequency bands, a small change will result in a large change in the sound levels, especially when considering the interference of the reflected wave with the direct wave.

In the following part, ten cases will be discussed in detail about the frequency changes by modification from four propagation paths to two propagation paths. Study will focus on the frequency bands ranged from $500 \mathrm{~Hz}$ to $2000 \mathrm{~Hz}$, since traffic noise often dominates by frequencies around $1000 \mathrm{~Hz}$ after A-weighting (Jonasson, 1971). In addition to frequency analysis of a point source for both models, overall absolute noise levels and insertion loss will be compared with both models for a one-lane line source by HNP 1.0. The geometry configurations are as follows: length of one-lane line source

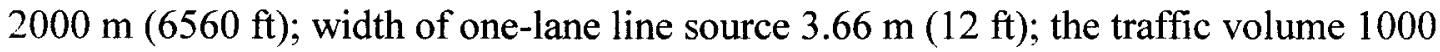
autos, $100 \mathrm{~km} / \mathrm{h}$ and $100 \mathrm{HT}, 100 \mathrm{~km} / \mathrm{h}$; roadway to the barrier distance $10 \mathrm{~m}(33 \mathrm{ft})$; 
barrier height $5 \mathrm{~m}(16 \mathrm{ft})$; receiver distance and height will be consistent with the data for frequency analysis, and the ground type is lawn in HNP.

\subsubsection{Comparisons of Varying Distances for the Receiver Position}

In order to evaluate the difference of insertion loss between the four propagation paths and the two propagation paths for varying receiver distance, we specify the

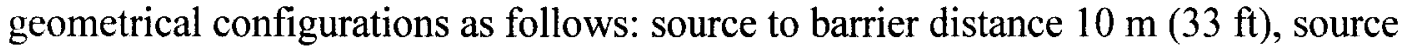
height $1.5 \mathrm{~m}(5 \mathrm{ft})$, barrier height $5 \mathrm{~m}(17 \mathrm{ft})$, receiver height $1.5 \mathrm{~m}(5 \mathrm{ft})$. Distances of 15 $\mathrm{m}(50 \mathrm{ft}), 30 \mathrm{~m}(100 \mathrm{ft}), 60 \mathrm{~m}(197 \mathrm{ft}), 120 \mathrm{~m}(394 \mathrm{ft})$ and $240 \mathrm{~m}(787 \mathrm{ft})$ are used for the receiver distance according to the generally highway noise barrier configurations.

\section{Case 1:}

In this case, receiver to barrier distance is $15 \mathrm{~m}(50 \mathrm{ft})$. Figure 7-36 shows the plot of insertion loss for the two propagation path model and the four propagation path model. 


\section{$\rightarrow-2$ propagation paths $\rightarrow-4$ propagation paths}

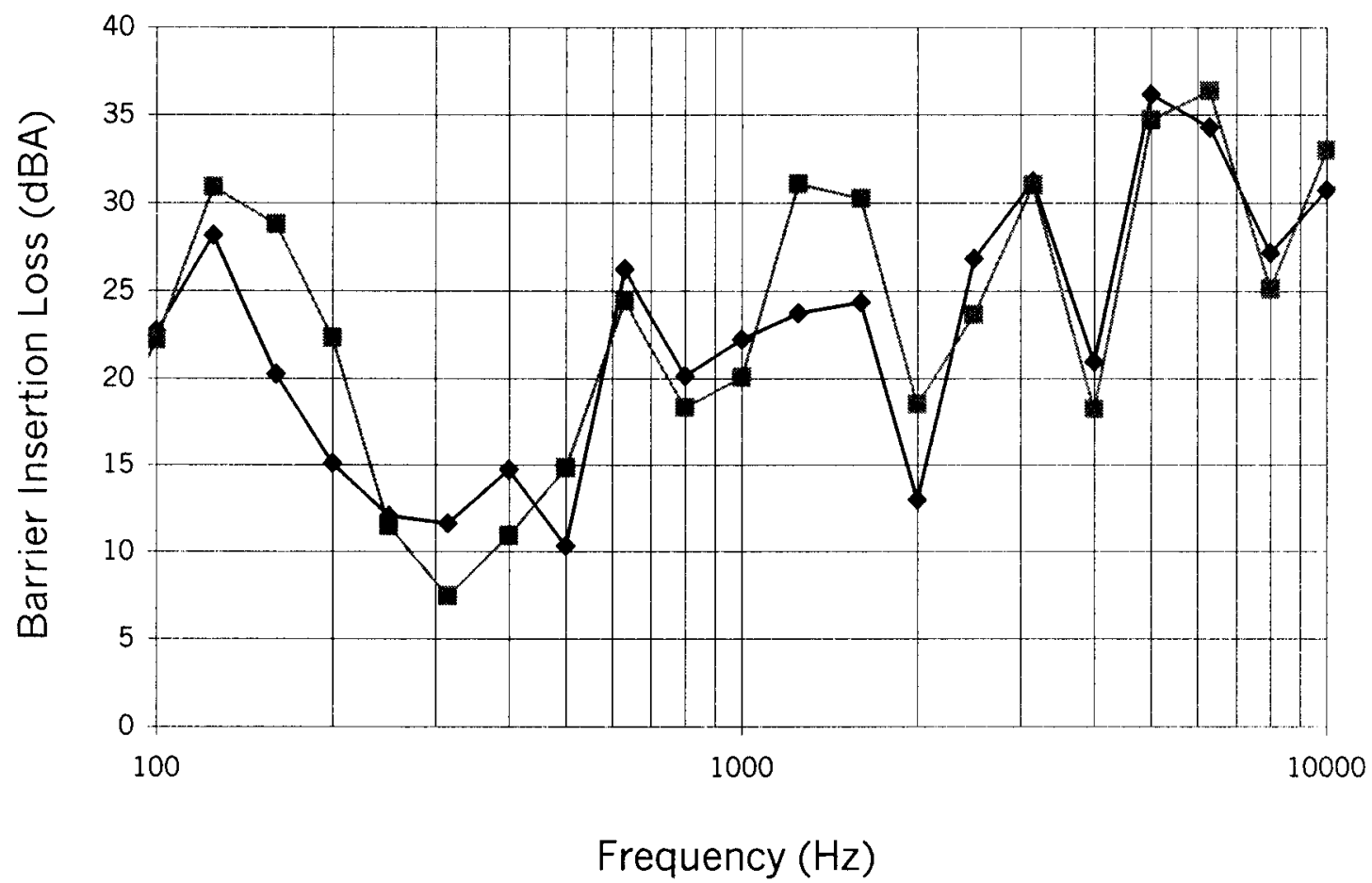

Figure 7-36. Comparison of insertion loss by 4 propagation paths and 2 propagation paths for case 1 , receiver to barrier distance $15 \mathrm{~m}(50 \mathrm{ft})$

As can be seen in Figure 7-36, when reducing from four propagation paths to two propagation paths, this modification will have greater influence on the low frequency bands and middle frequency bands than on the high frequency bands above $2000 \mathrm{~Hz}$. The first ground dip shifts from $315 \mathrm{~Hz}$ of 4 propagation path model to $500 \mathrm{~Hz}$ of 2 propagation path model. Large differences of insertion loss exist from $500 \mathrm{~Hz}$ frequency to $2000 \mathrm{~Hz}$ frequency. For example, 2 propagation path model under-predicts the insertion loss by $5.3 \mathrm{dBA}$ at $500 \mathrm{~Hz}$, over-predicts the insertion loss by $2.3 \mathrm{dBA}$ at 1000 $\mathrm{Hz}$ and under-predicts the insertion loss by $2.6 \mathrm{dBA}$ at $2000 \mathrm{~Hz}$, compared with 4 propagation path model. 
Case 2:

In this case, receiver to barrier distance is $30 \mathrm{~m}(100 \mathrm{ft})$. Figure 7-37 shows the plot of insertion loss for the two propagation paths model and the four propagation paths model.

As can be seen in Figure 7-37, when reducing from four propagation paths to two propagation paths, this modification will have greater influence on the low frequency bands and middle frequency bands than on the high frequency bands above $2000 \mathrm{~Hz}$. Both models predict the first ground dip at frequency of $500 \mathrm{~Hz}$. Large differences of insertions loss exist from $200 \mathrm{~Hz}$ frequency to $2000 \mathrm{~Hz}$ frequency. For example, 2 propagation path model over-predicts the insertion loss by $2.3 \mathrm{dBA}$ at $500 \mathrm{~Hz}$, overpredicts the insertion loss by $3.2 \mathrm{dBA}$ at $630 \mathrm{~Hz}$ and under-predicts the insertion loss by $3.8 \mathrm{dBA}$ at $1600 \mathrm{~Hz}$, compared with 4 propagation path model.

\section{Case 3:}

In this case, receiver to barrier distance is $60 \mathrm{~m}(197 \mathrm{ft})$. Figure 7-38 shows the plot of insertion loss for the two propagation paths model and the four propagation paths model.

As can be seen in Figure 7-38, when reducing from four propagation paths to two propagation paths, this modification will have large influence on all the frequency bands. 4 propagation path model predicts the largest ground dip at frequency of $800 \mathrm{~Hz}$. However, 2 propagation path model predicts the largest ground dip at frequency of 500 Hz. 2 propagation path model under-predicts the insertion loss by $4.0 \mathrm{dBA}$ at $500 \mathrm{~Hz}$, 
over-predicts the insertion loss by $3.0 \mathrm{dBA}$ at $1000 \mathrm{~Hz}$ and over-predicts the insertion loss by $5.1 \mathrm{dBA}$ at $1600 \mathrm{~Hz}$, compared with 4 propagation path model.
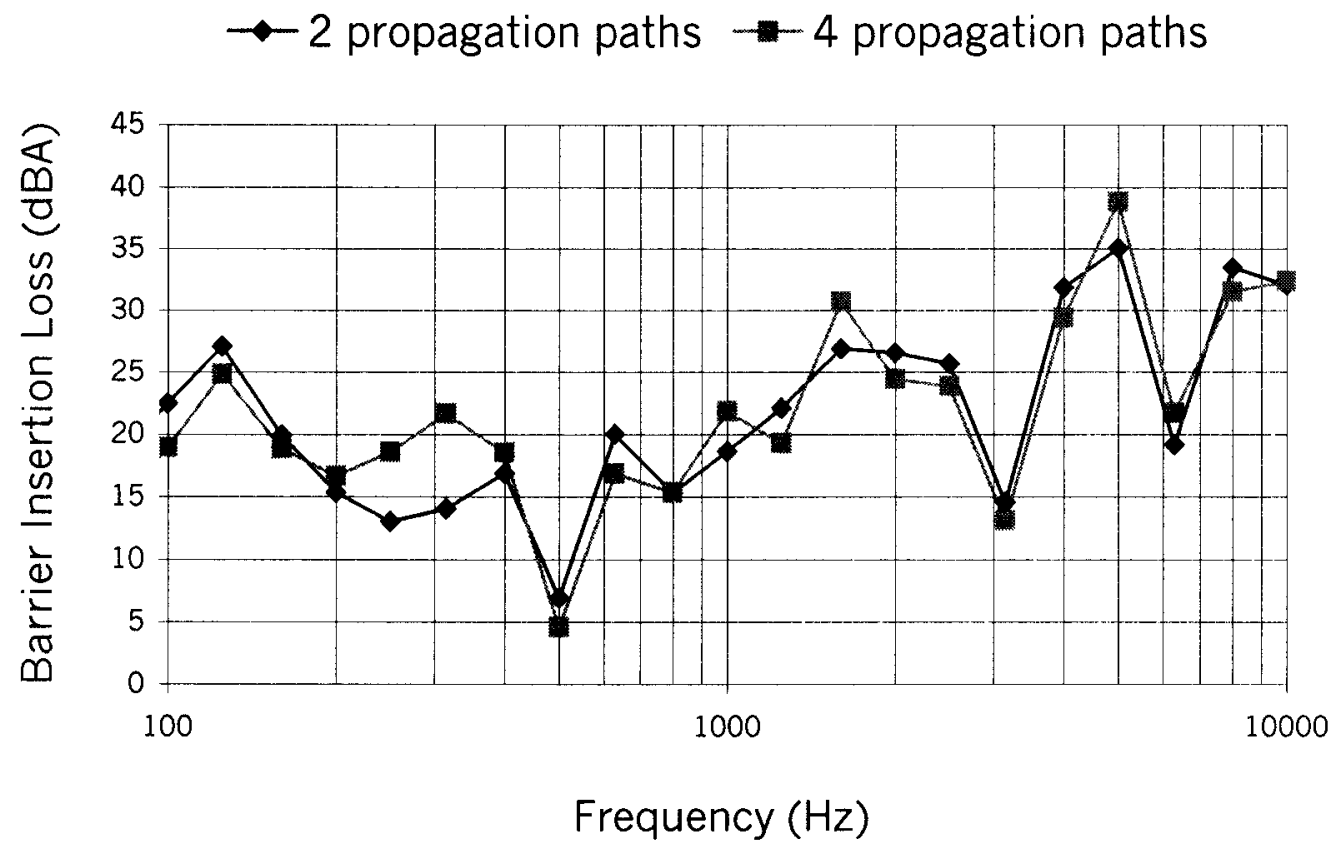

Figure 7-37. Comparison of insertion loss by 4 propagation paths and 2 propagation paths for case 2, receiver to barrier distance $30 \mathrm{~m}(100 \mathrm{ft})$

$\multimap 2$ propagation paths -4 propagation paths

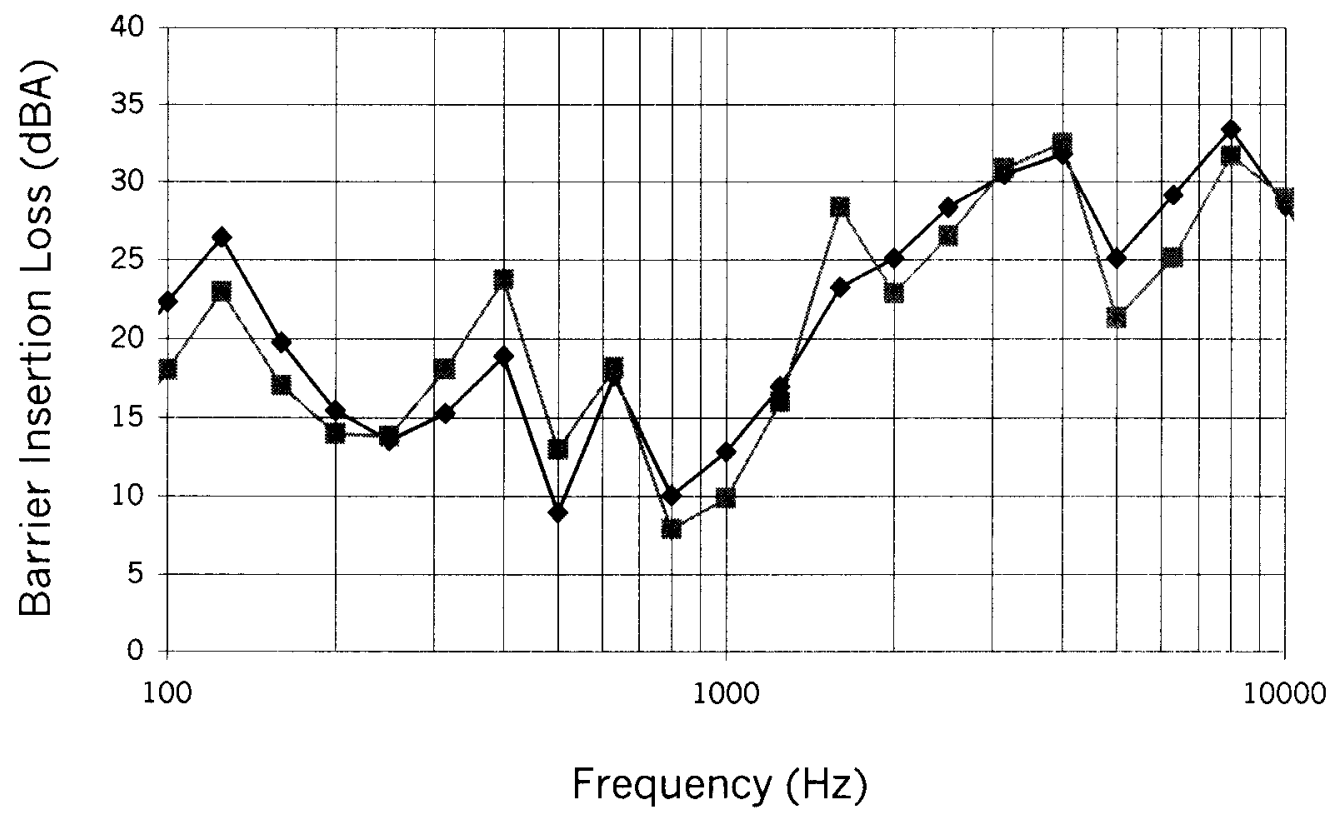

Figure 7-38. Comparison of insertion loss by 4 propagation paths and 2 propagation paths for case 3, receiver to barrier distance $60 \mathrm{~m}(197 \mathrm{ft})$ 
Case 4:

In this case, receiver to barrier distance is $120 \mathrm{~m}$ (394 ft). Figure 7-39 shows the plot of insertion loss for the two propagation paths model and the four propagation paths model.

As can be seen in Figure 7-39, when reducing from four propagation paths to two propagation paths, this modification will have large influence on all the frequency bands. Both models predict the largest ground dip at frequency of $1000 \mathrm{~Hz}$. However, 2 propagation path model under-predicts the insertion loss by $6.1 \mathrm{dBA}$ at $500 \mathrm{~Hz}$, overpredicts the insertion loss by $3.6 \mathrm{dBA}$ at $1250 \mathrm{~Hz}$ and over-predicts the insertion loss by $3.4 \mathrm{dBA}$ at $2000 \mathrm{~Hz}$, compared with 4 propagation path model.

\section{$\rightarrow 2$ propagation paths $\rightarrow-4$ propagation paths}

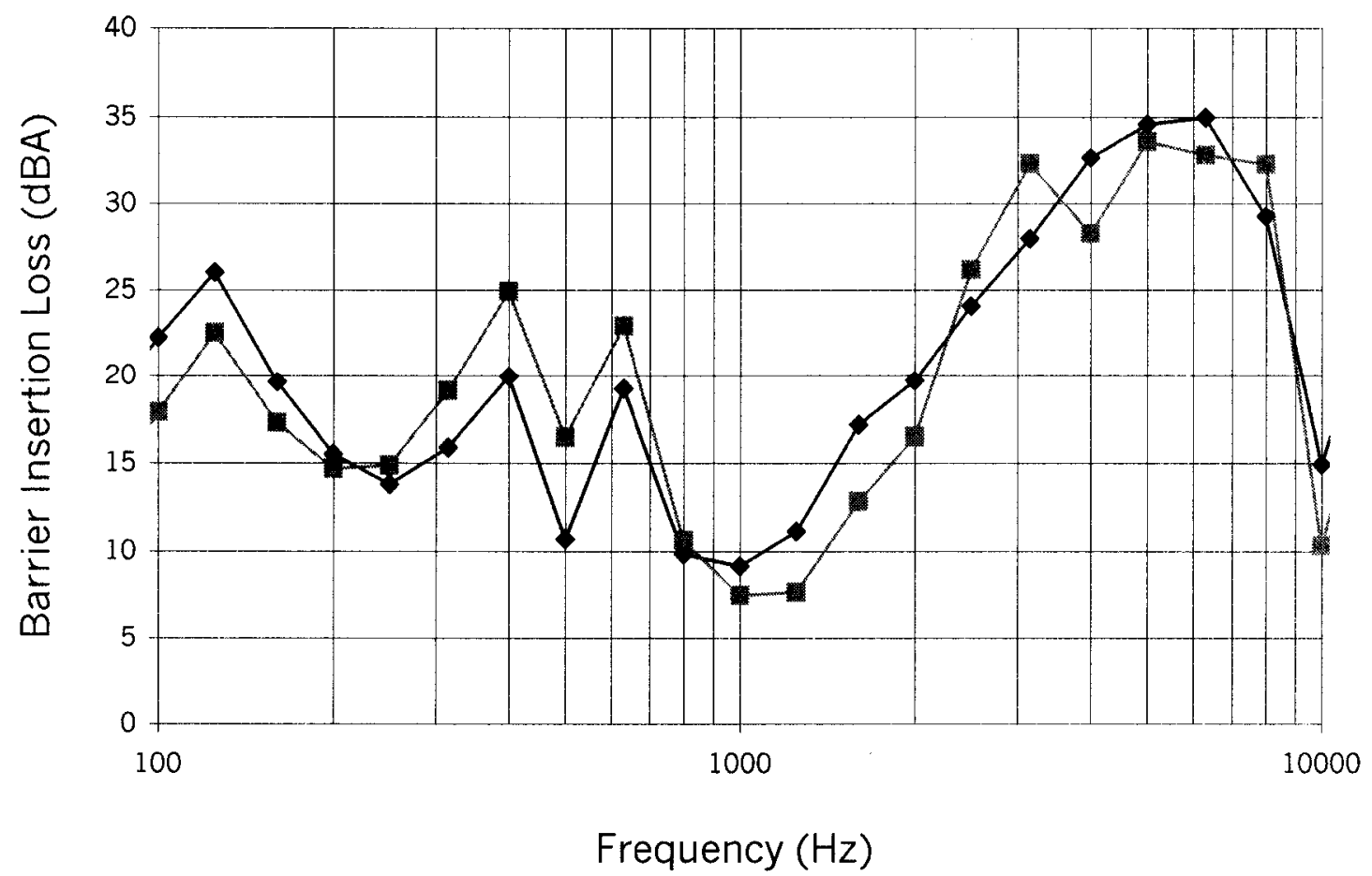

Figure 7-39. Comparison of insertion loss by 4 propagation paths and 2 propagation paths for case 4 , receiver to barrier distance $120 \mathrm{~m}(394 \mathrm{ft})$ 
Case 5:

In this case, receiver to barrier distance is $240 \mathrm{~m}(800 \mathrm{ft})$. Figure $7-40$ shows the plot of insertion loss for the two propagation paths model and the four propagation paths model.

As can be seen in Figure 7-40, when reducing from four propagation paths to two propagation paths, this modification will have large influence on all the frequency bands. Both models predict the largest ground dip at frequency of $1250 \mathrm{~Hz}$. However, 2 propagation path model under-predicts the insertion loss for most low and middle frequency bands as large as $9.5 \mathrm{dBA}$ at $500 \mathrm{~Hz}$. This is expected since two propagation path model does not account the ground attenuation behind the barrier. However, the ground attenuation plays a more important role with excess attenuation when the receiver getting further and further away from the barrier.

$\multimap-2$ propagation paths $\rightarrow-4$ propagation paths

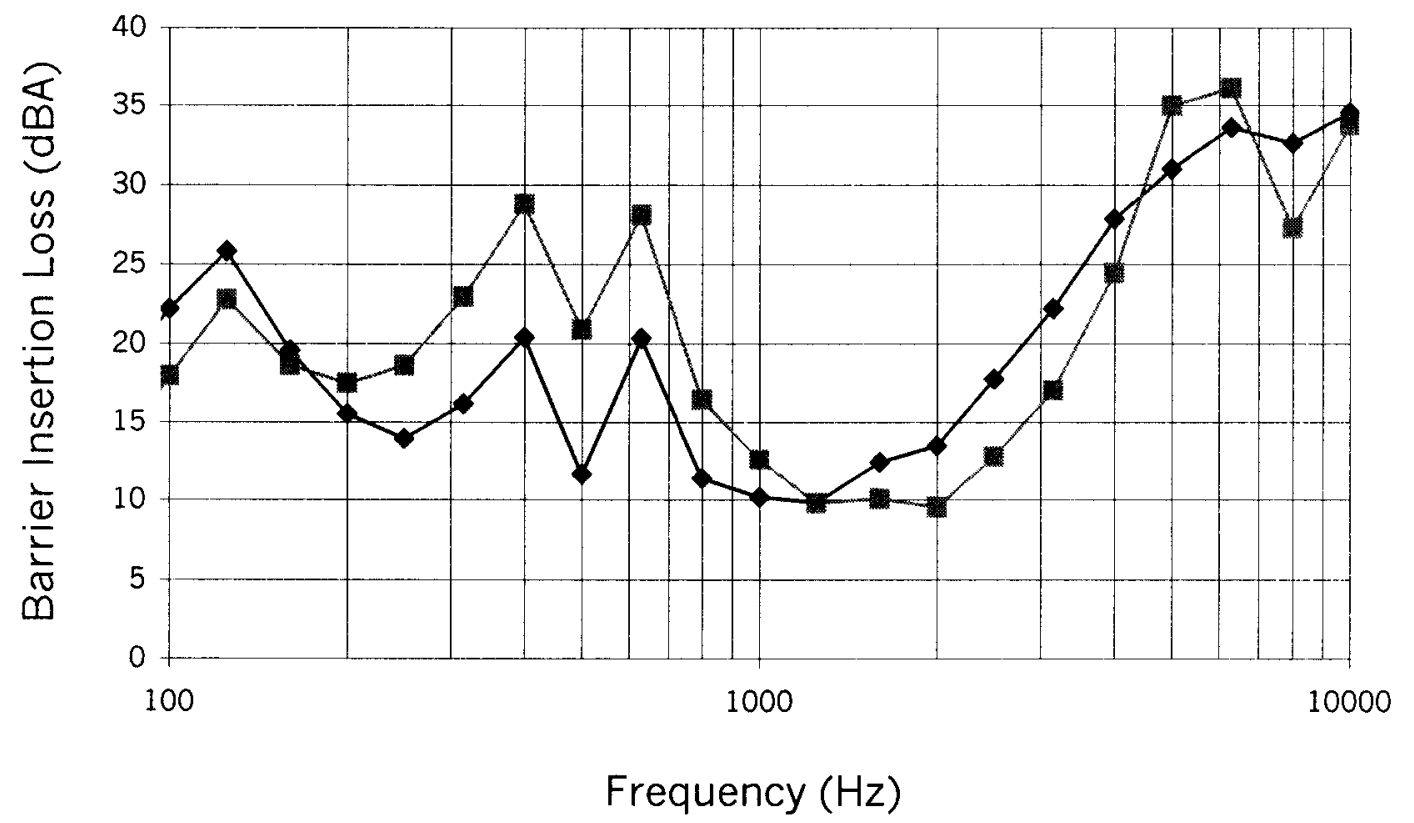

Figure 7-40. Comparison of insertion loss by 4 propagation paths and 2 propagation paths for case 5 , receiver to barrier distance $240 \mathrm{~m}(800 \mathrm{ft})$ 
Table 7-16. Comparison of absolute noise levels and insertion loss (dBA) of four propagation path model and two propagation path model by HNP with varying receiver distance at the height of $1.5 \mathrm{~m}(5 \mathrm{ft})$

\begin{tabular}{lllllll}
\hline Rec.distance from the barrier & $15 \mathrm{~m}$ & $30 \mathrm{~m}$ & $60 \mathrm{~m}$ & $120 \mathrm{~m}$ & $240 \mathrm{~m}$ \\
\hline 4 propagation paths & BEFORE & 66.8 & 63.3 & 59.2 & 54.0 & 47.3 \\
& AFTER & 55.2 & 53.2 & 50.5 & 46.2 & 39.8 \\
& IL & 11.6 & 10.1 & 8.7 & 7.8 & 7.5 \\
& & & & & & \\
2 propagation paths & BEFORE & 66.8 & 63.3 & 59.2 & 54.0 & 47.3 \\
& AFTER & 54.8 & 53.2 & 50.7 & 47.5 & 43.3 \\
& IL & 12.0 & 10.1 & 8.5 & 6.5 & 4.0 \\
\hline
\end{tabular}

Table 7-16 shows that when the receiver is close to the barrier, the insertion loss by two propagation path model is greater than that of two propagation path model. When the receiver is further away from the barrier, the insertion loss by two propagation path model is less than that of four propagation path model. For example, at $240 \mathrm{~m}(800 \mathrm{ft})$ away from the receiver, two propagation path model under-predicts the insertion loss by $3.5 \mathrm{dBA}$ compared with four propagation path model.

\subsubsection{Comparisons of Varying Heights for the Receiver Position}

In order to evaluate the difference of insertion loss between the four propagation paths and the two propagation paths with varying receiver heights, we specify the

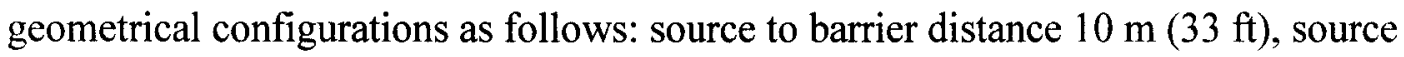
height $1.5 \mathrm{~m}(5 \mathrm{ft})$, barrier height $5 \mathrm{~m}(17 \mathrm{ft})$, receiver to barrier distance $15 \mathrm{~m}(50 \mathrm{ft})$. Heights of $0 \mathrm{~m}(0 \mathrm{ft}), 0.5 \mathrm{~m}(1.7 \mathrm{ft}), 1 \mathrm{~m}(3.3 \mathrm{ft}), 2 \mathrm{~m}(6.6 \mathrm{ft})$ and $4 \mathrm{~m}(13.2 \mathrm{ft})$ are used for the receiver heights according to the generally highway noise barrier configurations. 
Case 6:

In this case, receiver height is $0 \mathrm{~m}(0 \mathrm{ft})$. Figure 7-41 shows the plot of insertion loss for the two propagation paths model and the four propagation paths model.

As can be seen in Figure 7-41, when reducing from four propagation paths to two propagation paths, two propagation path model over-predicts the insertion loss almost on all the frequency bands, especially for low and middle frequency bands. Both models predict the largest ground dip at frequency of $500 \mathrm{~Hz}$. However, two propagation path model over-predicts the insertion loss by $4.5 \mathrm{dBA}$ at $500 \mathrm{~Hz}$, over-predicts the insertion loss by $3.5 \mathrm{dBA}$ at $1000 \mathrm{~Hz}$ and over-predicts the insertion loss by $3.4 \mathrm{dBA}$ at $2000 \mathrm{~Hz}$, compared with four propagation path model.

\section{$\rightarrow 2$ propagation paths $\rightarrow-4$ propagation paths}

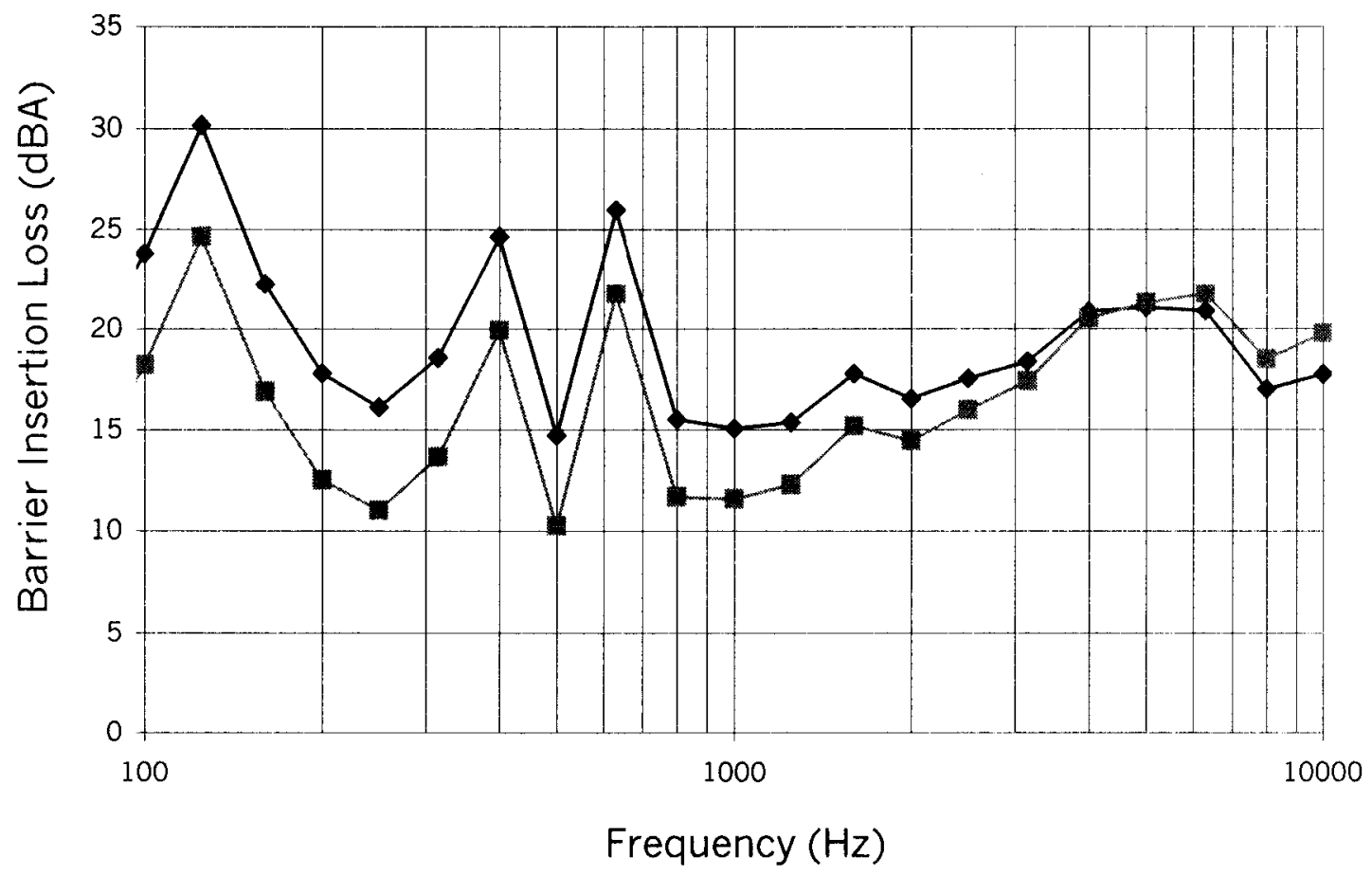

Figure 7-41. Comparison of insertion loss by 4 propagation paths and 2 propagation paths for case 6 , receiver height $0 \mathrm{~m}(0 \mathrm{ft})$ 
Case 7:

In this case, receiver height is $0.5 \mathrm{~m}(1.7 \mathrm{ft})$. Figure $7-42$ shows the plot of insertion loss for the two propagation paths model and the four propagation paths model.

As can be seen in Figure 7-42, when reducing from four propagation paths to two propagation paths, this modification will have large influence on low and middle frequency bands than on high frequency bands. Four propagation path model predicts the largest ground dip at the frequency of $800 \mathrm{~Hz}$ and two propagation path model predicts the largest ground dip at the frequency of $500 \mathrm{~Hz}$. two propagation path model underpredicts the insertion loss by $9.4 \mathrm{dBA}$ at $500 \mathrm{~Hz}$, over-predicts the insertion loss by 3.8 $\mathrm{dBA}$ at $1000 \mathrm{~Hz}$ and over-predicts the insertion loss by $3.1 \mathrm{dBA}$ at $2000 \mathrm{~Hz}$, compared with 4 propagation path model.

\section{$\rightarrow-2$ propagation paths $\rightarrow-4$ propagation paths}

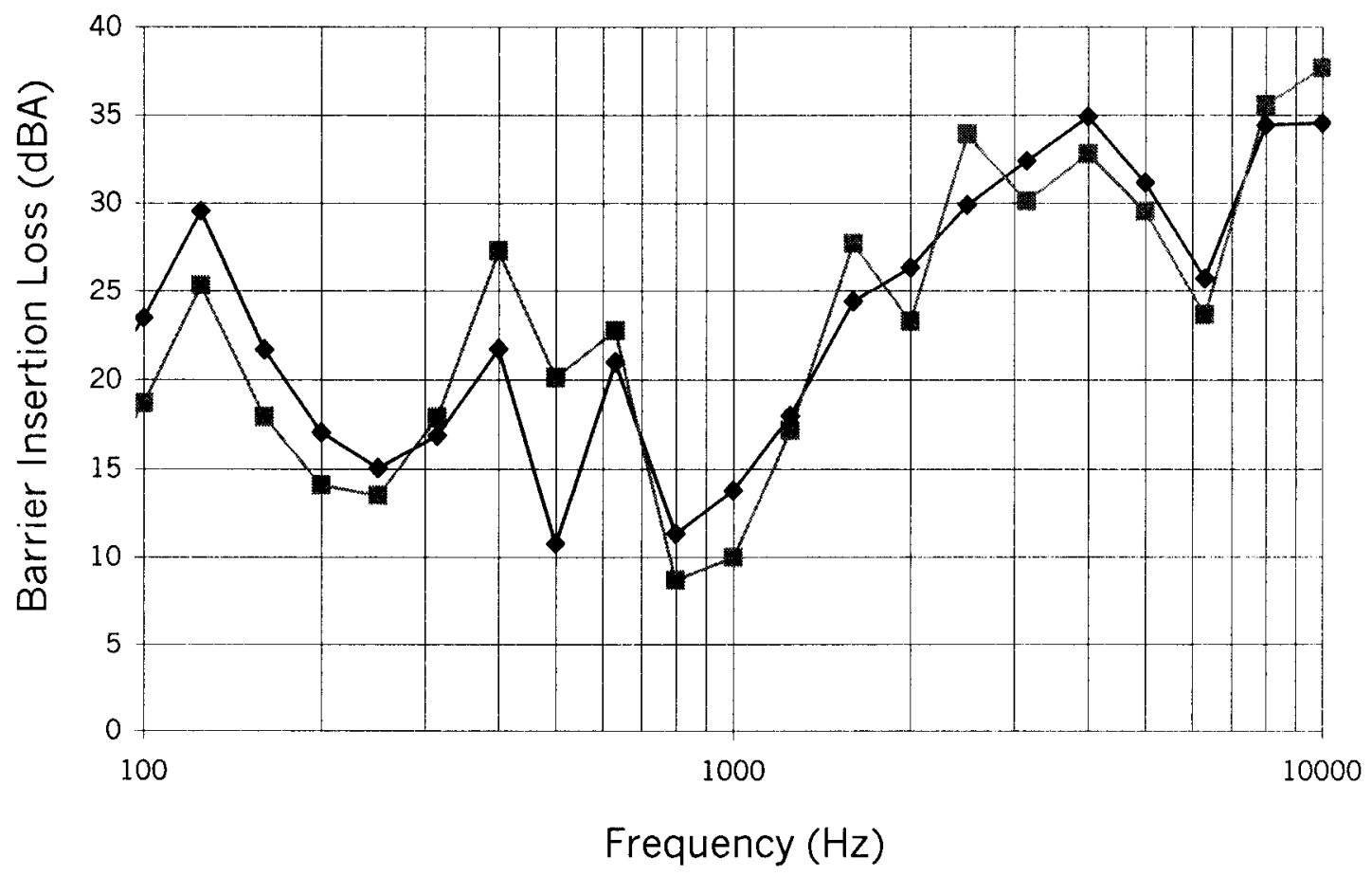

Figure 7-42. Comparison of insertion loss by 4 propagation paths and 2 propagation paths for case 7 , receiver height $0.5 \mathrm{~m}(1.7 \mathrm{ft})$ 


\section{Case 8:}

In this case, receiver height is $1.0 \mathrm{~m}(3.3 \mathrm{ft})$. Figure $7-43$ shows the plot of insertion loss for the two propagation paths model and the four propagation paths model.

As can be seen in Figure 7-43, when reducing from four propagation paths to two propagation paths, this modification will have large influence on low and middle frequency bands than on high frequency bands. Both models predict the largest ground dip at the frequency of $500 \mathrm{~Hz}$. Two propagation path model over-predicts the insertion loss by $4.6 \mathrm{dBA}$ at $500 \mathrm{~Hz}$, under-predicts the insertion loss by $7.5 \mathrm{dBA}$ at $800 \mathrm{~Hz}$ and over-predicts the insertion loss by $2.8 \mathrm{dBA}$ at $2000 \mathrm{~Hz}$, compared with four propagation path model.
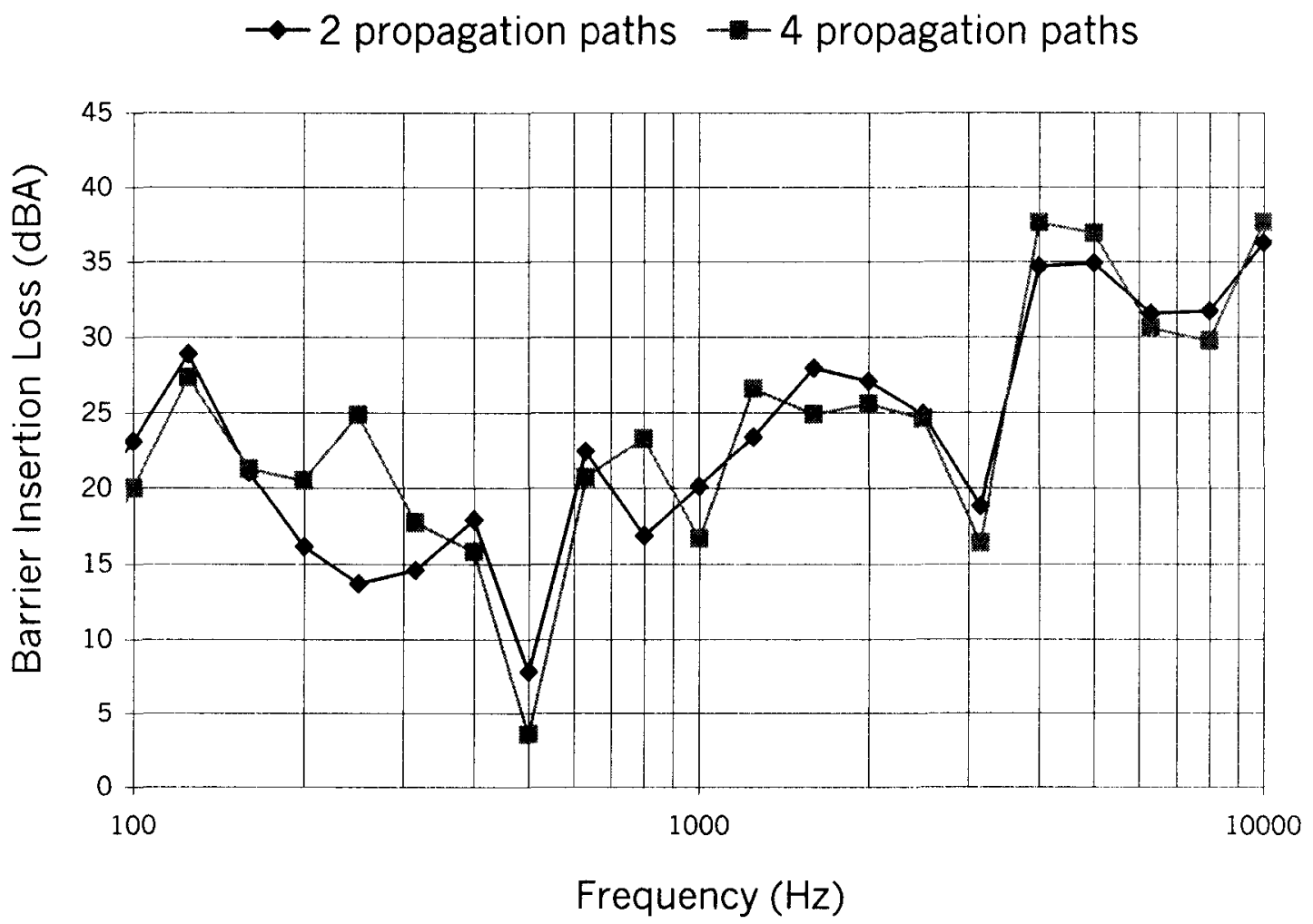

Figure 7-43. Comparison of insertion loss by 4 propagation paths and 2 propagation paths for case 8 , receiver height $1.0 \mathrm{~m}(3.3 \mathrm{ft})$ 
Case 9:

In this case, receiver height is $2.0 \mathrm{~m}(6.6 \mathrm{ft})$. Figure $7-44$ shows the plot of insertion loss for the two propagation paths model and the four propagation paths model.

As can be seen in Figure 7-44, when reducing from four propagation paths to two propagation paths, this modification will have large influence on low and middle frequency bands than on high frequency bands. Both models predict the largest ground dip at the frequency of $315 \mathrm{~Hz}$. Two propagation path model over-predicts the insertion loss by $3.2 \mathrm{dBA}$ at $500 \mathrm{~Hz}$, over-predicts the insertion loss by $3.6 \mathrm{dBA}$ at $800 \mathrm{~Hz}$ and under-predicts the insertion loss by $2.7 \mathrm{dBA}$ at $2000 \mathrm{~Hz}$, compared with four propagation path model.

$\multimap 2$ propagation paths -4 propagation paths

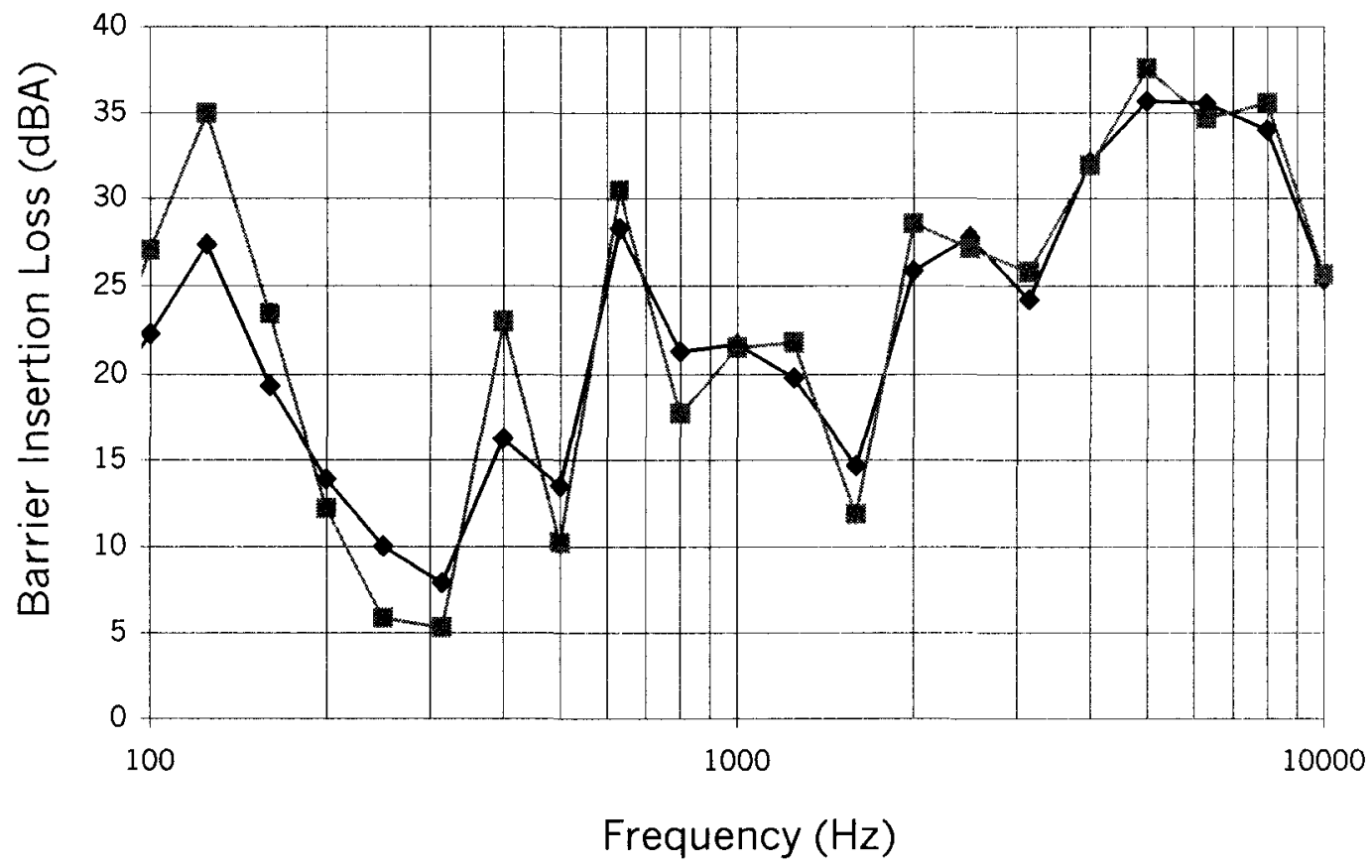

Figure 7-44. Comparison of insertion loss by 4 propagation paths and 2 propagation paths for case 9 , receiver height $2 \mathrm{~m}(6.6 \mathrm{ft})$ 
Case 10:

In this case, receiver height is $4.0 \mathrm{~m}(13.2 \mathrm{ft})$. Figure $7-45$ shows the plot of insertion loss for the two propagation paths model and the four propagation paths model.

As can be seen in Figure 7-45, when reducing from four propagation paths to two propagation paths, this modification will have small influence all the frequency bands. Both models predict the largest ground dip at the frequency of $250 \mathrm{~Hz}$.

$\rightarrow-2$ propagation paths $\rightarrow-4$ propagation paths

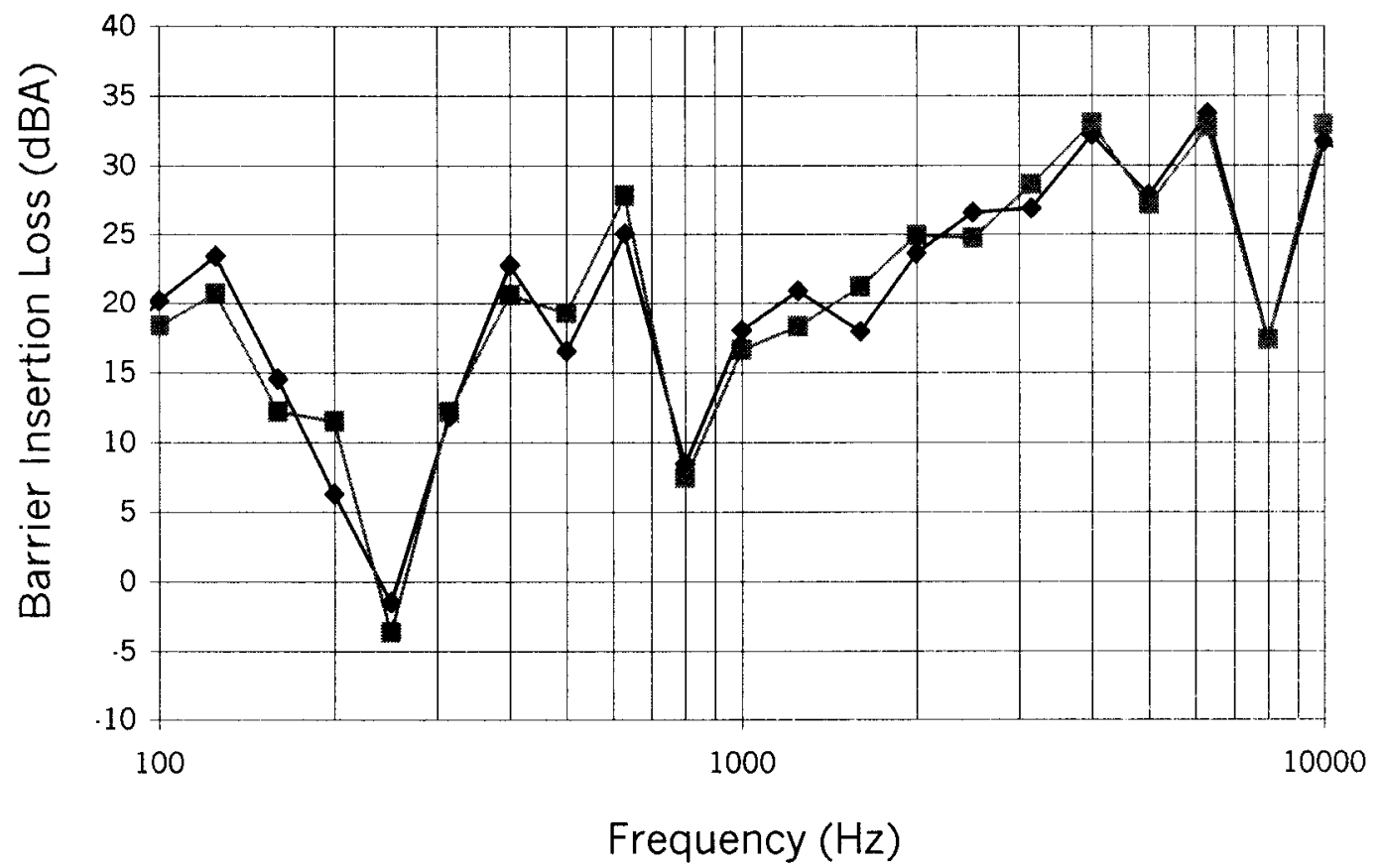

Figure 7-45. Comparison of insertion loss by 4 propagation paths and 2 propagation paths for case 10 , receiver height $4 \mathrm{~m}(13.2 \mathrm{ft})$ 
Table 7-17. Comparison of absolute noise levels and insertion loss (dBA) of four propagation path model and two propagation path model by HNP and TNM with varying receiver height at the distance of $15 \mathrm{~m}(50 \mathrm{ft})$ from the barrier

\begin{tabular}{|c|c|c|c|c|c|c|c|}
\hline & Receiver height & & $0 \mathrm{~m}$ & $0.5 \mathrm{~m}$ & $1.0 \mathrm{~m}$ & $2.0 \mathrm{~m}$ & $4.0 \mathrm{~m}$ \\
\hline \multirow[t]{6}{*}{ HNP 1.0} & \multirow{3}{*}{4 propagation paths } & BEFORE & 65.2 & 64.4 & 65.7 & 67.7 & 70.3 \\
\hline & & AFTER & 56 & 54.1 & 54.7 & 55.8 & 57.5 \\
\hline & & IL & 9.2 & 10.3 & 11 & 11.9 & 12.8 \\
\hline & \multirow[t]{3}{*}{2 propagation paths } & BEFORE & 65.2 & 64.4 & 65.7 & 67.7 & 70.3 \\
\hline & & AFTER & 53.8 & 54.1 & 54.4 & 55.2 & 57.2 \\
\hline & & IL & 11.4 & 10.3 & 11.3 & 12.5 & 13.1 \\
\hline \multirow[t]{3}{*}{ TNM 2.5} & \multirow[t]{3}{*}{2 propagation paths } & BEFORE & 65.6 & 65.3 & 67.2 & 70.1 & 71.4 \\
\hline & & AFTER & 55.9 & 54.2 & 54.8 & 55.9 & 56.9 \\
\hline & & IL & 9.7 & 11.1 & 12.4 & 14.2 & 14.5 \\
\hline
\end{tabular}

As can be seen in Table 7-17, when the receiver distance is $15 \mathrm{~m}(50 \mathrm{ft})$ from the barrier with varying height from $0 \mathrm{~m}(0 \mathrm{ft})$ to $4 \mathrm{~m}(13.2 \mathrm{ft})$, the insertion loss of HNP 1.0 ranges from $9.2 \mathrm{dBA}$ to $12.8 \mathrm{dBA}$ with 4 propagation path model, and the insertion loss ranges from $11.4 \mathrm{dBA}$ to $13.1 \mathrm{dBA}$ with 2 propagation path model in HNP 1.0, which is $0.3 \mathrm{dBA}$ to $2.2 \mathrm{dBA}$ over-prediction of insertion loss. In TNM 2.5, the insertion loss ranges from $9.7 \mathrm{dBA}$ to $14.5 \mathrm{dBA}$.

Figure 7-46 and Figure 7-47 are the graphic representation of insertion loss of four propagation path model and two propagation path model by HNP. Figure 7-48 denotes the difference of insertion loss between four propagation path model and two propagation path model. As can be seen in Figure 7-48, when the receiver is close to the barrier, inside $30 \mathrm{~m}$ (100 ft) from the barrier in this case, using two propagation path model will over-predict the insertion loss by as much as $2 \mathrm{dBA}$. When the receiver is further away from the barrier and is more close to the ground surface, using two 
propagation path model will under-predict the insertion loss by as much as $3 \mathrm{dBA}$, compared with using four propagation path model. When the receiver is further from the ground surface behind the barrier, the differences between two models get smaller. As a conclusion, from the perspective of achieving more accurate prediction of insertion loss, four propagation path model should be applied.

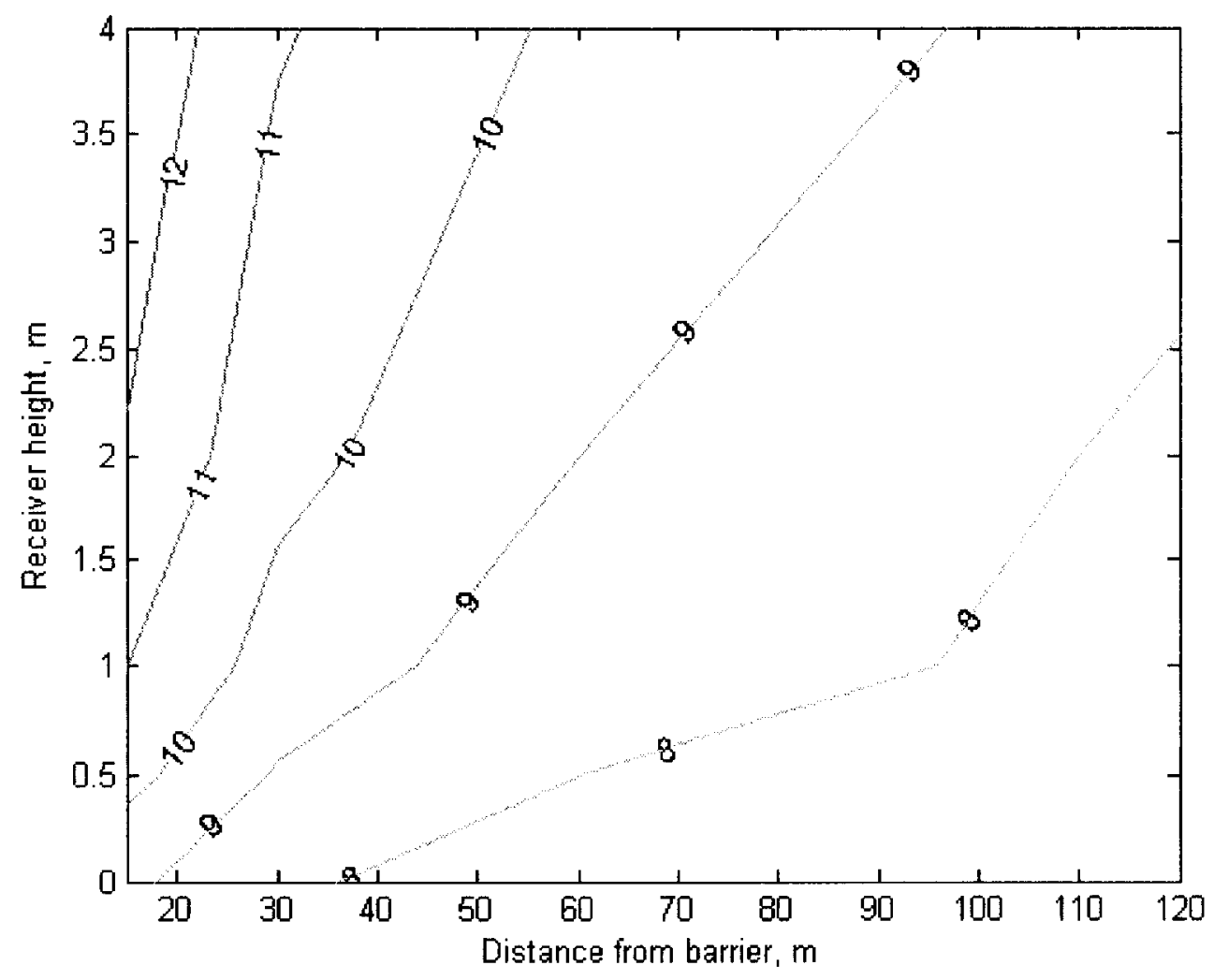

Figure 7-46. Contour of insertion loss (dBA) of four propagation path model by HNP with varying receiver heights and distances 


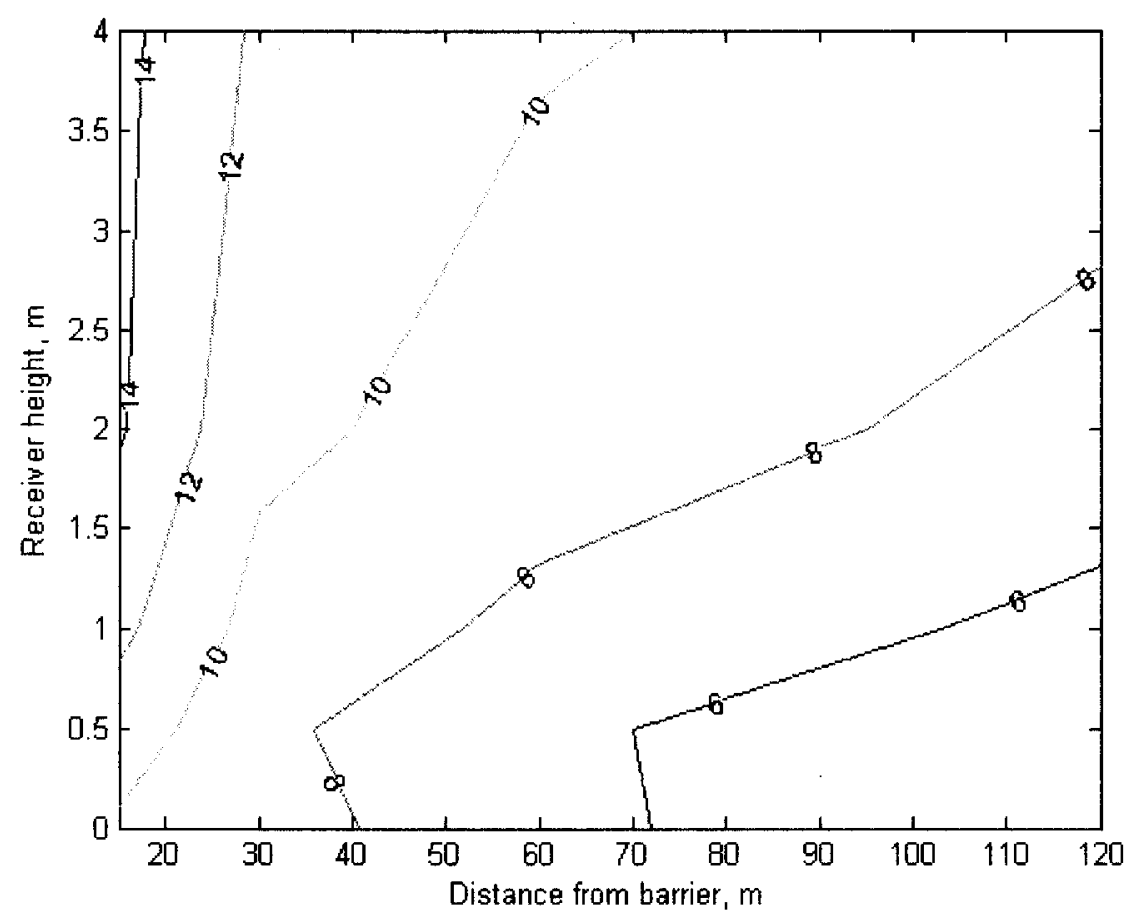

Figure 7-47. Contour of insertion loss (dBA) of two propagation path model by HNP with varying receiver heights and distances

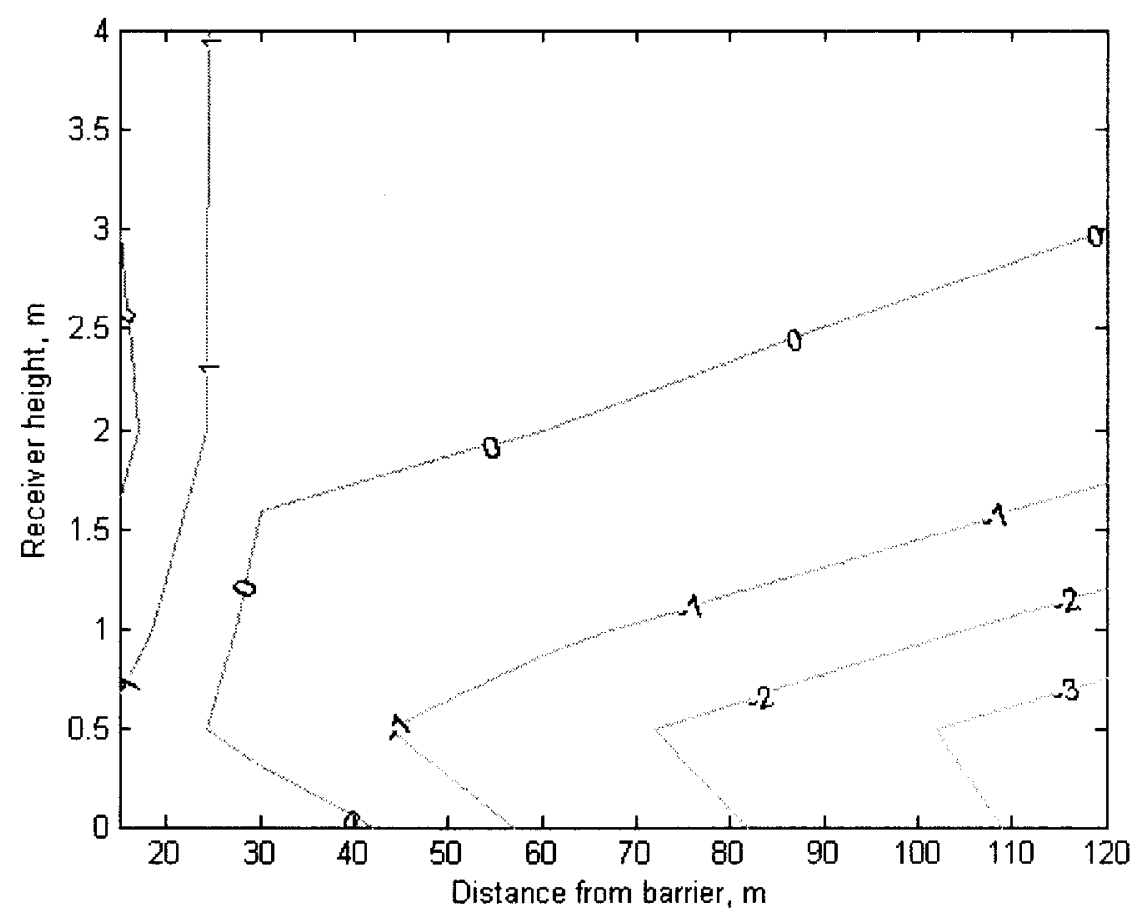

Figure 7-48. Contour of the difference of insertion loss (dBA) between four propagation path model and two propagation path model by HNP 


\section{CHPATER VIII}

\section{VALIDATION WITH THE FIELD MEASUREMENTS}

In this chapter, HNP 1.0 will be compared with TNM 2.5 , STAMINA 2.0 , and the field measurement data to evaluate the difference of the absolute noise levels and the insertion loss. Eight sites were modeled and compared, which included two sites (one barrier site: $01 \mathrm{KY}$. one open site: $04 \mathrm{IN}$ ) measured from June 2004 to October 2004 by the University of Louisville transportation team, and six sites (five barrier sites: 04CT, 05CT, 08CA, 09CA, and 11CA. one open site: 01MA) measured during 2002 by Volpe Center Acoustics Facility (VCAF), as shown in Appendix III. These sites were selected based on the simplicity for modeling in HNP 1.0 and a direct comparison with TNM 2.5 and STAMINA 2.0. HNP 1.0 used the original models developed by VCAF without any modifications. The VCAF field measurement data and input files of related TNM models were obtained by requesting a copy of the CD of Validation of FHWA's Traffic Noise Model (TNM): Phase 1. Comparisons were focused on the receivers that were $1.5 \mathrm{~m}(5 \mathrm{ft})$ above the ground surface for a general purpose. The measured noise levels were translated from $5 \mathrm{~min}$. into 1 hour noise levels; the $5 \mathrm{~min}$. traffic volume was summed up into the average hourly traffic volume. Results were compared from case to case and statistical comparisons of the absolute noise levels and the insertion loss were also made for the five barrier sites. 
In the following section, comparisons will be conducted from case to case. The absolute noise levels by three models will be compared with the measured data, the statistical analysis will be evaluated, and the insertion loss will also be compared.

\subsection{Case 1: site 01KY}

Table 8-1 shows the absolute noise levels with the barrier predicted by TNM 2.5 , STAMINA 2.0, and HNP 1.0, as well as their differences between each model and the measured data. At the reference receiver $1.5 \mathrm{~m}(5 \mathrm{ft})$ above the barrier, three models all under-predict the absolute noise levels, with $2.6 \mathrm{dBA}$ for STAMINA, $3.9 \mathrm{dBA}$ for HNP, and 4.1 dBA for TNM. It is partly because the roadway pavement type is "PCC"; more noise is generated compared to using "AVERAGE" pavement type in both TNM and HNP. For the receiver $15 \mathrm{~m}(50 \mathrm{ft})$ away from the barrier, STAMINA 2.0 predicts better absolute noise levels compared to TNM 2.5 and HNP 1.0 , with a $0.5 \mathrm{dBA}$ variation versus $3.3 \mathrm{dBA}$ and $2.7 \mathrm{dBA}$ under-predictions for TNM and HNP. For the receiver $30 \mathrm{~m}$ (100 ft) away from the barrier, STAMINA over-predicts absolute noise levels by $1.7 \mathrm{dBA}$,

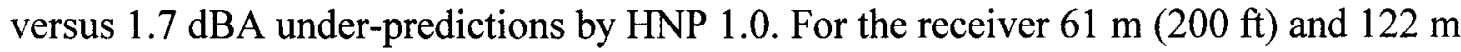
(400 ft) away from the barrier, STAMINA over-predicts the noise level by $4.1 \mathrm{dBA}$ and $6.3 \mathrm{dBA}$ consecutively, versus $0.3 \mathrm{dBA}$ and $3.8 \mathrm{dBA}$ over-predictions for TNM and 0.9 $\mathrm{dBA}$ and $3.2 \mathrm{dBA}$ over-predictions for HNP. 
Table 8-1. Comparisons of the absolute noise levels (dBA) by TNM, STAMINA, and HNP for site $01 \mathrm{KY}$

\begin{tabular}{llllllll}
\hline $\begin{array}{l}\text { Mic } \\
\text { Location }\end{array}$ & $\begin{array}{l}\text { Measured } \\
\text { levels }\end{array}$ & $\begin{array}{l}\text { Predicted } \\
\text { TNM 2.5 }\end{array}$ & $\begin{array}{l}\text { Predicted } \\
\text { STA 2.0 }\end{array}$ & $\begin{array}{l}\text { Predicted } \\
\text { HNP 1.0 }\end{array}$ & $\begin{array}{l}\text { TNM - } \\
\text { Measured }\end{array}$ & $\begin{array}{l}\text { STA - } \\
\text { Measured }\end{array}$ & $\begin{array}{l}\text { HNP - } \\
\text { Measured }\end{array}$ \\
\hline Ref & 82.7 & 78.6 & 80.1 & 78.8 & -4.1 & -2.6 & -3.9 \\
$50 \mathrm{ft}$ & 67.9 & 64.6 & 68.4 & 65.2 & -3.3 & 0.5 & -2.7 \\
$100 \mathrm{ft}$ & 66.8 & 64.0 & 68.5 & 65.1 & -2.8 & 1.7 & -1.7 \\
$200 \mathrm{ft}$ & 63.0 & 63.3 & 67.1 & 63.9 & 0.3 & 4.1 & 0.9 \\
$400 \mathrm{ft}$ & 58.0 & 61.8 & 64.3 & 61.2 & 3.8 & 6.3 & 3.2 \\
\hline
\end{tabular}

Table 8-2. Statistical analysis for site $01 \mathrm{KY}$

\begin{tabular}{lccc}
\hline & TNM & STAMINA & HNP \\
\hline Mean & -1.2 & 2.0 & -0.84 \\
Standard Deviation & 3.3 & 3.4 & 2.9 \\
Range & 7.9 & 8.9 & 7.1 \\
Minimum & -4.1 & -2.6 & -3.9 \\
Maximum & 3.8 & 6.3 & 3.2 \\
\hline
\end{tabular}

Table 8-2 gives the descriptive statistics for table 8-1. It shows that HNP performs the best in four out of the five statistical categories evaluated. STAMINA 2.0 has the best value for minimum error of $-2.6 \mathrm{dBA}$, versus $-4.1 \mathrm{dBA}$ for TNM 2.5, and $-3.9 \mathrm{dBA}$ for HNP 1.0. It can be seen that, on the average, HNP has an error of $-0.84 \mathrm{dBA}$ while TNM 2.5 generates an error of $-1.2 \mathrm{dBA}$ under-predictions and STAMINA 2.0 was $2 \mathrm{dBA}$ over-predictions respectively.

Table 8-3. Comparisons of the insertion loss (dBA) by TNM, STAMINA, and HNP for the site $01 \mathrm{KY}$

\begin{tabular}{llllllll}
\hline $\begin{array}{l}\text { Mic } \\
\text { Location }\end{array}$ & $\begin{array}{l}\text { Measured } \\
\text { IL }\end{array}$ & $\begin{array}{l}\text { Predicted } \\
\text { TNM 2.5 }\end{array}$ & $\begin{array}{l}\text { Predicted } \\
\text { STA 2.0 }\end{array}$ & $\begin{array}{l}\text { Predicted } \\
\text { HNP 1.0 }\end{array}$ & $\begin{array}{l}\text { TNM - } \\
\text { Measured }\end{array}$ & $\begin{array}{l}\text { STA - } \\
\text { Measured }\end{array}$ & $\begin{array}{l}\text { HNP - } \\
\text { Measured }\end{array}$ \\
\hline $50 \mathrm{ft}$ & 6.4 & 11.7 & 6.4 & 6.5 & 5.3 & 0 & 0.1 \\
$100 \mathrm{ft}$ & 5.5 & 10.7 & 4.4 & 5.1 & 5.2 & -1.1 & -0.4 \\
$200 \mathrm{ft}$ & 4.5 & 8.5 & 3.0 & 4.3 & 4.0 & -1.5 & -0.2 \\
$400 \mathrm{ft}$ & 4.7 & 6.2 & 2.1 & 4.6 & 1.5 & -2.6 & -0.1 \\
\hline
\end{tabular}


As to the insertion loss comparisons shown in Table 8-3, STAMNA and HNP out-perform TNM compared to the indirect field measurements of insertion loss at the equivalent site. The errors range of insertion loss for STAMINA is from $-2.6 \mathrm{dBA}$ to 0 $\mathrm{dBA}$, with an average under-prediction error of $-1.3 \mathrm{dBA}$. TNM largely over-predicts the insertion loss compared to the measured insertion loss, ranging from $1.5 \mathrm{dBA}$ to $5.3 \mathrm{dBA}$, with an average over-prediction error of $4.0 \mathrm{dBA}$. HNP 1.0 predicts the most accurate insertion loss. The errors range from $-0.4 \mathrm{dBA}$ to $0.1 \mathrm{dBA}$, with an average error of 0.1 dBA.

Table 8-4 shows the comparisons of predicted insertion loss by HNP 1.0 with 4 propagation path model and HNP 1.0 with 2 propagation path model, with the measured insertion loss at the site $01 \mathrm{KY}$. As can be seen, 4 propagation path model predicts better insertion loss than 2 propagation path model. Compared with predicted insertion loss with 4 propagation paths, predicted insertion loss of HNP with 2 propagation paths overpredicts the insertion loss in the near barrier distances, less than $30 \mathrm{~m}$ (approximately 100 $\mathrm{ft}$ ) and under-predicts the insertion loss in the distance far from the barrier. For example, at the $15 \mathrm{~m}(50 \mathrm{ft})$ away from the barrier, 2 propagation path model generates $0.5 \mathrm{dBA}$ over-predictions compared with the measured insertion loss, and it is $0.1 \mathrm{dBA}$ overpredictions for 4 propagation path model. At the $122 \mathrm{~m}(400 \mathrm{ft})$ away from the barrier, 2 propagation path model generates $-0.9 \mathrm{dBA}$ under-predictions compared with the measured insertion loss, and it is $-0.1 \mathrm{dBA}$ under-predictions for 4 propagation path model. Since 2 propagation path model does not consider the ground effect behind the barrier, it will over-estimate the ground effect in the near distance and under-estimate the ground effect in the far distance from the barrier. This is also consistent with the 
theoretical analysis of frequency spectrum and predicted insertion loss of a single-line source in Chapter VII. Therefore, 2 propagation path model will not be considered further Table 8-4. Comparisons of predicted insertion loss by HNP 1.0 with 4 propagation path model and 2 propagation path model, with the measured insertion loss at the site $01 \mathrm{KY}$

\begin{tabular}{ccccc}
\hline $\begin{array}{l}\text { Mic } \\
\text { Location }\end{array}$ & $\begin{array}{c}\text { Measured } \\
\text { IL }\end{array}$ & $\begin{array}{l}\text { Predicted HNP with 4 } \\
\text { propagation paths }\end{array}$ & $\begin{array}{l}\text { Predicted HNP with 2 } \\
\text { propagation paths }\end{array}$ & $\begin{array}{l}\text { Difference of insertion } \\
\text { loss (2 path - 4 path) }\end{array}$ \\
\hline $50 \mathrm{ft}$ & 6.4 & 6.5 & 6.9 & 0.4 \\
$100 \mathrm{ft}$ & 5.5 & 5.1 & 5.1 & 0 \\
$200 \mathrm{ft}$ & 4.5 & 4.3 & 4.1 & -0.2 \\
$400 \mathrm{ft}$ & 4.7 & 4.6 & 3.8 & -0.8 \\
\hline
\end{tabular}

\subsection{Case 2: site 04IN}

Table 8-5. Comparisons of the absolute noise levels (dBA) by TNM, STAMINA, and HNP for the site $04 \mathrm{IN}$

\begin{tabular}{llllllll}
\hline $\begin{array}{l}\text { Mic } \\
\text { Location }\end{array}$ & $\begin{array}{l}\text { Measured } \\
\text { levels }\end{array}$ & $\begin{array}{l}\text { Predicted } \\
\text { TNM 2.5 }\end{array}$ & $\begin{array}{l}\text { Predicted } \\
\text { STA 2.0 }\end{array}$ & $\begin{array}{l}\text { Predicted } \\
\text { HNP 1.0 }\end{array}$ & $\begin{array}{l}\text { TNM - } \\
\text { Measured }\end{array}$ & $\begin{array}{l}\text { STA - } \\
\text { Measured }\end{array}$ & $\begin{array}{l}\text { HNP - } \\
\text { Measured }\end{array}$ \\
\hline $50 \mathrm{ft}$ & 66.5 & 67.1 & 67.2 & 65.8 & 0.6 & 0.7 & -0.7 \\
$100 \mathrm{ft}$ & 60 & 62.3 & 62.7 & 59.2 & 2.3 & 2.7 & -0.8 \\
$200 \mathrm{ft}$ & 53.6 & 56.6 & 58.6 & 53.6 & 3 & 5 & 0 \\
\hline
\end{tabular}

Table 8-5 shows the absolute noise levels in open space predicted by TNM 2.5 , STAMINA 2.0, and HNP 1.0, as well as their differences between each model and the measured data. For all the receivers, TNM 2.5 and STAMINA 2.0 over-predict the noise levels. HNP 1.0 slightly under-predicts the absolute noise levels. The errors for TNM 2.5 range from $0.6 \mathrm{dBA}$ to $3 \mathrm{dBA}$, with an average over-prediction error of $2.0 \mathrm{dBA}$. The errors for STAMINA range from $0.7 \mathrm{dBA}$ to $5 \mathrm{dBA}$, with an average over-prediction error of $2.8 \mathrm{dBA}$. The errors for HNP 1.0 range from $-0.8 \mathrm{dBA}$ to $0 \mathrm{dBA}$, with an average under-prediction error of $-0.5 \mathrm{dBA}$. 


\subsection{Case 3: site 04CT}

The field measurement data and input files of related TNM models from case 3 to case 8 were obtained from VCAF. The TNM models are then translated into HNP and STAMINA without modifications. The predicted absolute noise levels of TNM, STAMINA and HNP are compared to the measured data. In addition, the predicted insertion loss by TNM, STAMINA and HNP are compared with each other, since indirect insertion loss was not measured by VCAF.

Table 8-6. Comparisons of the absolute noise levels (dBA) by TNM, STAMINA, and HNP for the site 04CT

\begin{tabular}{llllllll}
\hline $\begin{array}{l}\text { Mic } \\
\text { Location }\end{array}$ & $\begin{array}{l}\text { Measured } \\
\text { levels }\end{array}$ & $\begin{array}{l}\text { Predicted } \\
\text { TNM 2.5 }\end{array}$ & $\begin{array}{l}\text { Predicted } \\
\text { STA 2.0 }\end{array}$ & $\begin{array}{l}\text { Predicted } \\
\text { HNP 1.0 }\end{array}$ & $\begin{array}{l}\text { TNM - } \\
\text { Measured }\end{array}$ & $\begin{array}{l}\text { STA - } \\
\text { Measured }\end{array}$ & $\begin{array}{l}\text { HNP - } \\
\text { Measured }\end{array}$ \\
\hline Ref & 79.2 & 78.5 & 78.8 & 79 & -0.7 & -0.4 & -0.2 \\
$56 \mathrm{ft}$ & 62.9 & 60.5 & 62.8 & 62.5 & -2.4 & -0.1 & -0.4 \\
$125 \mathrm{ft}$ & 62.4 & 59.7 & 62.4 & 61.1 & -2.7 & 0 & -1.3 \\
$200 \mathrm{ft}$ & 62 & 59 & 61.7 & 60.1 & -3 & -0.3 & -1.9 \\
\hline
\end{tabular}

Table 8-6 shows the absolute noise levels with barrier predicted by TNM 2.5 , STAMINA 2.0, and HNP 1.0, as well as their differences between each model and the measured data. At the reference receiver $1.5 \mathrm{~m}(5 \mathrm{ft})$ above the barrier, the predicted noise levels all are within $1 \mathrm{dBA}$ variations, with $-0.4 \mathrm{dBA}$ for STAMINA $2.0,-0.2 \mathrm{dBA}$ for HNP 1.0, and $-0.7 \mathrm{dBA}$ for TNM 2.5. For all the receivers $1.5 \mathrm{~m}(5 \mathrm{ft})$ above the ground behind the barrier, TNM 2.5 under-predicts the sound levels, ranging from -3.0 dBA to -2.4 dBA. STAMINA 2.0 and HNP 1.0 out-perform TNM 2.5 for all these receivers, ranging from $-0.3 \mathrm{dBA}$ to $0 \mathrm{dBA}$ for STAMINA 2.0 , and ranging from -1.9 to -

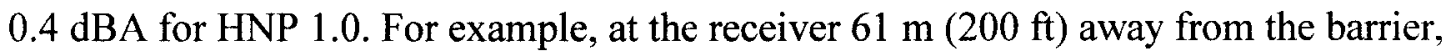
TNM has $-3.0 \mathrm{dBA}$ under-predictions, versus $-0.3 \mathrm{dBA}$ variations for STAMINA 2.0 and $-1.9 \mathrm{dBA}$ variations for HNP 1.0. 
Table 8-7. Statistical analysis for the site 04CT

\begin{tabular}{lccc}
\hline & TNM & STAMINA & HNP \\
\hline Mean & -2.20 & -0.20 & -0.95 \\
Standard Deviation & 1.03 & 0.18 & 0.79 \\
Range & 2.30 & 0.40 & 1.70 \\
Minimum & -3.00 & -0.40 & -1.90 \\
Maximum & -0.70 & 0.00 & -0.20 \\
\hline
\end{tabular}

Table 8-7 shows that STAMINA performs the best in five out of the five statistical categories evaluated. It can be seen that, on the average, STAMINA has an error of $-0.20 \mathrm{dBA}$ while TNM 2.5 is $-2.2 \mathrm{~dB}$ and $\mathrm{HNP}$ is $-0.95 \mathrm{dBA}$ respectively.

Table 8-8. Comparisons of the insertion loss (dBA) by TNM, STAMINA, and HNP for the site $04 \mathrm{CT}$

\begin{tabular}{llllll}
\hline Mic & Predicted & Predicted & Predicted & TNM - & TNM - \\
Location & TNM 2.5 & STA 2.0 & HNP 1.0 & STA & HNP \\
\hline $56 \mathrm{ft}$ & 11.8 & 12.3 & 11.3 & -0.5 & 0.5 \\
$125 \mathrm{ft}$ & 9.4 & 10.3 & 8.6 & -0.9 & 0.8 \\
$200 \mathrm{ft}$ & 7.9 & 9.0 & 6.3 & -1.1 & 1.6 \\
Average & 9.7 & 10.5 & 8.7 & -0.8 & 1.0 \\
\hline
\end{tabular}

As to the insertion loss comparison, the predicted insertion loss for TNM 2.5 ranges from $7.9 \mathrm{dBA}$ to $11.8 \mathrm{dBA}$, versus $9.0 \mathrm{dBA}$ to $12.3 \mathrm{dBA}$ for STAMINA 2.0 and 6.3 dBA to $11.3 \mathrm{dBA}$ for HNP. On the average, TNM 2.5 predicts $0.8 \mathrm{dBA}$ less insertion loss than STAMINA 2.0 and $1.0 \mathrm{dBA}$ greater insertion loss than HNP 1.0 in this case. 


\subsection{Case 4: site 05CT}

Table 8-9. Comparisons of the absolute noise levels by TNM, STAMINA, and HNP for site $05 \mathrm{CT}$

\begin{tabular}{llllllll}
\hline $\begin{array}{l}\text { Mic } \\
\text { Location }\end{array}$ & $\begin{array}{c}\text { Measured } \\
\text { levels }\end{array}$ & $\begin{array}{l}\text { Predicted } \\
\text { TNM 2.5 }\end{array}$ & $\begin{array}{l}\text { Predicted } \\
\text { STA 2.0 }\end{array}$ & $\begin{array}{l}\text { Predicted } \\
\text { HNP 1.0 }\end{array}$ & $\begin{array}{l}\text { TNM - } \\
\text { Measured }\end{array}$ & $\begin{array}{l}\text { STA - } \\
\text { Measured }\end{array}$ & $\begin{array}{l}\text { HNP - } \\
\text { Measured }\end{array}$ \\
\hline Ref & 80.5 & 80.3 & 81.3 & 81.9 & -0.2 & 0.8 & 1.4 \\
$50 \mathrm{ft}$ & 60.6 & 61.3 & 64.5 & 61.5 & 0.7 & 3.9 & 0.9 \\
$100 \mathrm{ft}$ & 58.9 & 59.7 & 63.4 & 59.9 & 0.8 & 4.5 & 1.0 \\
$150 \mathrm{ft}$ & 58.1 & 58.5 & 62.3 & 58.8 & 0.4 & 4.2 & 0.7 \\
\hline
\end{tabular}

Table 8-9 shows the absolute noise levels with the barrier predicted by TNM 2.5 , STAMINA 2.0, and HNP 1.0, as well as their differences between each model and the measured data. At the reference receiver $1.5 \mathrm{~m}(5 \mathrm{ft})$ above the barrier, the predicted noise levels for TNM and STAMINA are within $1 \mathrm{dBA}$ variations, with $0.8 \mathrm{dBA}$ for STAMINA 2.0, $-0.2 \mathrm{dBA}$ for TNM 2.5 , and $1.4 \mathrm{dBA}$ over-predictions for HNP 1.0. For all the receivers $1.5 \mathrm{~m}(5 \mathrm{ft})$ above the ground behind the barrier, TNM 2.5 and HNP 1.0 out-perform STAMINA 2.0. The errors for TNM 2.5 range from $0.4 \mathrm{dBA}$ to $0.8 \mathrm{dBA}$ and HNP 1.0 ranges from $0.7 \mathrm{dBA}$ to $1.0 \mathrm{dBA}$. The over-predictions for STAMINA 2.0 range from $3.9 \mathrm{dBA}$ to $4.5 \mathrm{dBA}$.

Table 8-10. Statistical analysis for the site $05 \mathrm{CT}$

\begin{tabular}{lccc}
\hline & TNM & STAMINA & HNP \\
\hline Mean & 0.43 & 3.35 & 1.00 \\
Standard Deviation & 0.45 & 1.72 & 0.29 \\
Range & 1.00 & 3.70 & 0.70 \\
Minimum & -0.20 & 0.80 & 0.70 \\
Maximum & 0.80 & 4.50 & 1.40 \\
\hline
\end{tabular}

Table 8-10 shows that TNM performs the best in three out of the five statistical categories evaluated. HNP has the best value for error range and standard deviation, with $0.7 \mathrm{dBA}$ and $0.29 \mathrm{dBA}$ respectively, versus $1.0 \mathrm{dBA}$ and $0.45 \mathrm{dBA}$ for TNM 2.5 , and 3.7 
$\mathrm{dBA}$ and $1.72 \mathrm{dBA}$ for STAMINA. It can be seen that, on the average, STAMINA has an error of $3.35 \mathrm{dBA}$ over-predictions while TNM 2.5 is $0.43 \mathrm{dBA}$ over-predictions and HNP is $1.0 \mathrm{dBA}$ over-prediction respectively.

Table 8-11. Comparisons of the insertion loss (dBA) by TNM, STAMINA, and HNP for the site 05CT

\begin{tabular}{llllll}
\hline $\begin{array}{l}\text { Mic } \\
\text { Location }\end{array}$ & $\begin{array}{l}\text { Predicted } \\
\text { TNM 2.5 }\end{array}$ & $\begin{array}{l}\text { Predicted } \\
\text { STA 2.0 }\end{array}$ & $\begin{array}{l}\text { Predicted } \\
\text { HNP 1.0 }\end{array}$ & $\begin{array}{l}\text { TNM }- \\
\text { STA }\end{array}$ & $\begin{array}{l}\text { TNM - } \\
\text { HNP }\end{array}$ \\
\hline $50 \mathrm{ft}$ & 15.0 & 11.2 & 14.2 & 3.8 & 0.8 \\
$100 \mathrm{ft}$ & 14.0 & 9.2 & 11.5 & 4.8 & 2.5 \\
$150 \mathrm{ft}$ & 13.0 & 8.3 & 9.4 & 4.7 & 3.6 \\
Average & 14.0 & 9.6 & 11.7 & 4.4 & 2.3 \\
\hline
\end{tabular}

As to the insertion loss comparison, the predicted insertion loss for TNM 2.5

ranges from $13.0 \mathrm{dBA}$ to $15.0 \mathrm{dBA}$, versus $8.3 \mathrm{dBA}$ to $11.2 \mathrm{dBA}$ for STAMINA 2.0 and 9.4 dBA to $14.2 \mathrm{dBA}$ for HNP 1.0. On the average, TNM 2.5 predicts $4.4 \mathrm{dBA}$ greater insertion loss than STAMINA 2.0 and $2.3 \mathrm{dBA}$ greater insertion loss than HNP 1.0 in this case.

\subsection{Case 5: site 08CA}

Table 8-12. Comparisons of the absolute noise levels (dBA) by TNM, STAMINA, and HNP for the site $08 \mathrm{CA}$

\begin{tabular}{llllllll}
\hline $\begin{array}{l}\text { Mic } \\
\text { Location }\end{array}$ & $\begin{array}{l}\text { Measured } \\
\text { levels }\end{array}$ & $\begin{array}{l}\text { Predicted } \\
\text { TNM 2.5 }\end{array}$ & $\begin{array}{l}\text { Predicted } \\
\text { STA 2.0 }\end{array}$ & $\begin{array}{l}\text { Predicted } \\
\text { HNP 1.0 }\end{array}$ & $\begin{array}{l}\text { TNM - } \\
\text { Measured }\end{array}$ & $\begin{array}{l}\text { STA - } \\
\text { Measured }\end{array}$ & $\begin{array}{l}\text { HNP - } \\
\text { Measured }\end{array}$ \\
\hline Ref & 80.5 & 79.1 & 78.3 & 80.0 & -1.4 & -2.2 & -0.5 \\
$50 \mathrm{ft}$ & 65.7 & 65.0 & 68.8 & 64.8 & -0.7 & 3.1 & -0.9 \\
$200 \mathrm{ft}$ & 65.3 & 63.3 & 68.0 & 63.3 & -2.0 & 2.7 & -2.0 \\
$300 \mathrm{ft}$ & 62.8 & 61.7 & 66.7 & 61.7 & -1.1 & 3.9 & -1.1 \\
\hline
\end{tabular}

Table 8-12 shows the absolute noise levels with the barrier predicted by TNM 2.5 , STAMINA 2.0, and HNP 1.0, as well as their differences between each model and the 
measured data. At the reference receiver $1.5 \mathrm{~m}(5 \mathrm{ft})$ above the barrier, the predicted noise levels for $\mathrm{HNP}$ are within $1 \mathrm{dBA}$ variations, with $-0.5 \mathrm{~dB}$ versus $-1.4 \mathrm{dBA}$ for TNM 2.5, and $-2.2 \mathrm{dBA}$ for STAMINA 2.0. For all the receivers $1.5 \mathrm{~m}(5 \mathrm{ft})$ above the ground behind the barrier, TNM 2.5 and HNP 1.0 out-perform STAMINA 2.0 for all these receivers. The errors for TNM 2.5 range from $-2 \mathrm{dBA}$ to $-0.7 \mathrm{dBA}$ and HNP ranges from $-2.0 \mathrm{dBA}$ to $-0.5 \mathrm{dBA}$. The over-predictions for STAMINA range from $2.7 \mathrm{dBA}$ to 3.9 dBA.

Table 8-13. Statistical analysis for the site $08 \mathrm{CA}$

\begin{tabular}{lccc}
\hline & TNM & STAMINA & HNP \\
\hline Mean & -1.30 & 1.88 & -1.13 \\
Standard Deviation & 0.55 & 2.76 & 0.63 \\
Range & 1.30 & 6.10 & 1.50 \\
Minimum & -2.00 & -2.20 & -2.00 \\
Maximum & -0.70 & 3.90 & -0.50 \\
\hline
\end{tabular}

Table 8-13 shows that HNP performs the best in three out of the five statistical categories evaluated. TNM also performs the best in three out of the five statistics evaluated. It can be seen that, on the average, the mean for TNM 2.5 and HNP 1.0 are $1.3 \mathrm{dBA}$ and $-1.13 \mathrm{dBA}$ respectively. STAMINA averaged $1.88 \mathrm{dBA}$ over-predictions compared to the field measured data.

Table 8-14. Comparisons of the insertion loss (dBA) by TNM, STAMINA, and HNP for the site $08 \mathrm{CA}$

\begin{tabular}{llllll}
\hline $\begin{array}{l}\text { Mic } \\
\text { Location }\end{array}$ & $\begin{array}{l}\text { Predicted } \\
\text { TNM 2.5 }\end{array}$ & $\begin{array}{l}\text { Predicted } \\
\text { STA 2.0 }\end{array}$ & $\begin{array}{l}\text { Predicted } \\
\text { HNP 1.0 }\end{array}$ & $\begin{array}{l}\text { TNM - } \\
\text { STA }\end{array}$ & $\begin{array}{l}\text { TNM - } \\
\text { HNP }\end{array}$ \\
\hline $50 \mathrm{ft}$ & 11.9 & 7.0 & 12.1 & 4.9 & -0.2 \\
$200 \mathrm{ft}$ & 9.2 & 3.4 & 8.8 & 5.8 & 0.4 \\
$300 \mathrm{ft}$ & 8.7 & 2.7 & 7.6 & 6.0 & 1.1 \\
Average & 9.9 & 4.4 & 9.5 & 5.6 & 0.4 \\
\hline
\end{tabular}


As to the insertion loss comparison, the predicted insertion loss for TNM 2.5 ranges from $8.7 \mathrm{dBA}$ to $11.9 \mathrm{dBA}$, with an average value of $9.9 \mathrm{dBA}$. The predicted insertion loss for STAMINA 2.0 ranges from $2.7 \mathrm{dBA}$ to $7 \mathrm{dBA}$, with an average value of 4.4 dBA. The predicted insertion loss for HNP 1.0 ranges from $7.6 \mathrm{dBA}$ to $12.1 \mathrm{dBA}$, with an average value of $9.5 \mathrm{dBA}$ in this case.

\subsection{Case 6: site 09CA}

Table 8-15. Comparisons of the absolute noise levels (dBA) by TNM, STAMINA, and HNP for the site 09CA

\begin{tabular}{llllllll}
\hline $\begin{array}{l}\text { Mic } \\
\text { Location }\end{array}$ & $\begin{array}{l}\text { Measured } \\
\text { levels }\end{array}$ & $\begin{array}{l}\text { Predicted } \\
\text { TNM 2.5 }\end{array}$ & $\begin{array}{l}\text { Predicted } \\
\text { STA 2.0 }\end{array}$ & $\begin{array}{l}\text { Predicted } \\
\text { HNP 1.0 }\end{array}$ & $\begin{array}{l}\text { TNM - } \\
\text { Measured }\end{array}$ & $\begin{array}{l}\text { STA - } \\
\text { Measured }\end{array}$ & $\begin{array}{l}\text { HNP - } \\
\text { Measured }\end{array}$ \\
\hline Ref & 80.3 & 79.4 & 81.3 & 83.7 & -0.9 & 1.0 & 3.4 \\
$55 \mathrm{ft}$ & 61.3 & 58.5 & 60.9 & 61.9 & -2.8 & -0.4 & 0.6 \\
$100 \mathrm{ft}$ & 61.3 & 57.3 & 60.9 & 60.1 & -4.0 & -0.4 & -1.2 \\
$200 \mathrm{ft}$ & 61.3 & 55.8 & 60.2 & 59.3 & -5.5 & -1.1 & -2.0 \\
\hline
\end{tabular}

Table 8-15 shows the absolute noise levels with the barrier predicted by TNM 2.5 , STAMINA 2.0, and HNP 1.0, as well as their differences between each model and the measured data. At the reference receiver $1.5 \mathrm{~m}(5 \mathrm{ft})$ above the barrier, the predicted noise levels for TNM and STAMINA are within $1 \mathrm{dBA}$ variations, with $-0.9 \mathrm{dBA}$ and 1.0 dBA respectively. HNP over-predicted the noise level by $3.4 \mathrm{dBA}$ at the reference receiver. For all the receivers $1.5 \mathrm{~m}(5 \mathrm{ft})$ above the ground behind the barrier, STAMINA 2.0 and HNP 1.0 out-perform TNM 2.5 for all these receivers. The errors for STAMINA 2.0 range from $-1.1 \mathrm{dBA}$ to $-0.4 \mathrm{dBA}$ and HNP 1.0 ranges from $-2 \mathrm{dBA}$ to 0.6 dBA. The under-prediction for TNM 2.5 ranges from $-5.5 \mathrm{dBA}$ to $-2.8 \mathrm{dBA}$. 
Table 8-16. Statistical analysis for the site $09 \mathrm{CA}$

\begin{tabular}{lccc}
\hline & TNM & STAMINA & HNP \\
\hline Mean & -3.30 & -0.23 & 0.20 \\
Standard Deviation & 1.94 & 0.88 & 2.39 \\
Range & 4.60 & 2.10 & 5.40 \\
Minimum & -5.50 & -1.10 & -2.00 \\
Maximum & -0.90 & 1.00 & 3.40 \\
\hline
\end{tabular}

Table 8-16 shows that STAMINA 2.0 performs the best in three out of the five statistics evaluated. HNP 1.0 has the best value for error mean and TNM has the best value for maximum error. It can be seen that, on the average, the mean for STAMINA 2.0 and HNP 1.0 are within $0.5 \mathrm{dBA}$, which is $-0.23 \mathrm{dBA}$ for STAMINA 2.0 and $0.20 \mathrm{dBA}$ for HNP 1.0 respectively. TNM averages $-3.3 \mathrm{dBA}$ under-predictions compared to the field measured data.

\subsection{Case 7: site 11CA}

Table 8-17. Comparisons of the absolute noise levels (dBA) by TNM, STAMINA, and HNP for the site $11 \mathrm{CA}$

\begin{tabular}{llllllll}
\hline $\begin{array}{l}\text { Mic } \\
\text { Location }\end{array}$ & $\begin{array}{l}\text { Measured } \\
\text { levels }\end{array}$ & $\begin{array}{l}\text { Predicted } \\
\text { TNM 2.5 }\end{array}$ & $\begin{array}{l}\text { Predicted } \\
\text { STA 2.0 }\end{array}$ & $\begin{array}{l}\text { Predicted } \\
\text { HNP 1.0 }\end{array}$ & $\begin{array}{l}\text { TNM - } \\
\text { Measured }\end{array}$ & $\begin{array}{l}\text { STA - } \\
\text { Measured }\end{array}$ & $\begin{array}{l}\text { HNP - } \\
\text { Measured }\end{array}$ \\
\hline Ref & 80.4 & 78.6 & 77.3 & 80.1 & -1.8 & -3.1 & -0.3 \\
$50 \mathrm{ft}$ & 59.9 & 60.2 & 61.7 & 59.8 & 0.3 & 1.8 & -0.1 \\
$100 \mathrm{ft}$ & 59.8 & 59.6 & 61.7 & 59.5 & -0.2 & 1.9 & -0.3 \\
\hline
\end{tabular}

Table 8-17 shows the absolute noise levels with the barrier predicted by TNM 2.5 , STAMINA 2.0, and HNP 1.0, as well as their differences between each model and the measured data. At the reference receiver $1.5 \mathrm{~m}(5 \mathrm{ft})$ above the barrier, the predicted noise levels for HNP 1.0 are within $1 \mathrm{dBA}$ variations, with $-0.3 \mathrm{dBA}$ versus $-1.8 \mathrm{dBA}$ for

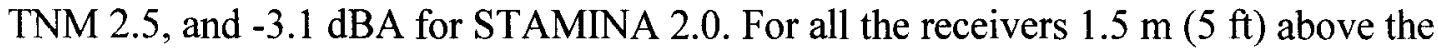
ground behind the barrier, TNM 2.5 and HNP 1.0 out-perform STAMINA 2.0 for all 
these receivers. The errors for TNM 2.5 range from $-0.2 \mathrm{dBA}$ to $0.3 \mathrm{dBA}$ and HNP 1.0 ranges from $-0.3 \mathrm{dBA}$ to $-0.1 \mathrm{dBA}$. The over-predictions for STAMINA 2.0 range from $1.8 \mathrm{dBA}$ to $1.9 \mathrm{dBA}$.

Table 8-18. Statistical analysis for the site 11CA

\begin{tabular}{lccc}
\hline & TNM & STAMINA & HNP \\
\hline Mean & -0.57 & 0.20 & -0.23 \\
Standard Deviation & 1.10 & 2.86 & 0.12 \\
Range & 2.10 & 5.00 & 0.20 \\
Minimum & -1.80 & -3.10 & -0.30 \\
Maximum & 0.30 & 1.90 & -0.10 \\
\hline
\end{tabular}

Table 8-18 shows that HNP 1.0 performs the best in four out of the five statistical categories evaluated, including minimum error, maximum error, standard deviation and error range. STAMINA 2.0 has the best value for error mean. It can be seen that, on the average, the error mean for TNM 2.5 is $-0.57 \mathrm{dBA}$, versus $0.20 \mathrm{dBA}$ for STAMINA 2.0 , and $-0.23 \mathrm{dBA}$ for HNP 1.0 .

Table 8-19. Comparisons of the insertion loss (dBA) by TNM, STAMINA, and HNP for the site $11 \mathrm{CA}$

\begin{tabular}{llllll}
\hline $\begin{array}{l}\text { Mic } \\
\text { Location }\end{array}$ & $\begin{array}{l}\text { Predicted } \\
\text { TNM 2.5 }\end{array}$ & $\begin{array}{l}\text { Predicted } \\
\text { STA 2.0 }\end{array}$ & $\begin{array}{l}\text { Predicted } \\
\text { HNP 1.0 }\end{array}$ & $\begin{array}{l}\text { TNM - } \\
\text { STA }\end{array}$ & $\begin{array}{l}\text { TNM - } \\
\text { HNP }\end{array}$ \\
\hline $50 \mathrm{ft}$ & 15.6 & 11.0 & 11.0 & 4.6 & 4.6 \\
$100 \mathrm{ft}$ & 14.3 & 9.1 & 9.3 & 5.2 & 5.0 \\
Average & 15.0 & 10.1 & 10.2 & 4.9 & 4.8 \\
\hline
\end{tabular}

As to the insertion loss comparison, the predicted insertion loss for TNM 2.5 ranges from $14.3 \mathrm{dBA}$ to $15.6 \mathrm{dBA}$, with an average value of $15.0 \mathrm{dBA}$. The predicted insertion loss for STAMINA 2.0 ranges from $9.1 \mathrm{dBA}$ to $11 \mathrm{dBA}$, with an average value of $10.1 \mathrm{dBA}$. The predicted insertion loss for HNP 1.0 ranges from $9.3 \mathrm{dBA}$ to $11.0 \mathrm{dBA}$, 
with an average value of $10.2 \mathrm{dBA}$ in this case. TNM 2.5 has an average $4.9 \mathrm{dBA}$ greater insertion loss than STAMINA 2.0 and $4.8 \mathrm{dBA}$ greater insertion loss than HNP 1.0.

\subsection{Case 8: site 01MA}

Table 8-20. Comparisons of the absolute noise levels (dBA) by TNM, STAMINA, and HNP for the site 01MA

\begin{tabular}{llllllll}
\hline $\begin{array}{l}\text { Mic } \\
\text { Location }\end{array}$ & $\begin{array}{l}\text { Measured } \\
\text { levels }\end{array}$ & $\begin{array}{l}\text { Predicted } \\
\text { TNM 2.5 }\end{array}$ & $\begin{array}{l}\text { Predicted } \\
\text { STA 2.0 }\end{array}$ & $\begin{array}{l}\text { Predicted } \\
\text { HNP 1.0 }\end{array}$ & $\begin{array}{l}\text { TNM - } \\
\text { Measured }\end{array}$ & $\begin{array}{l}\text { STA - } \\
\text { Measured }\end{array}$ & $\begin{array}{l}\text { HNP - } \\
\text { Measured }\end{array}$ \\
\hline $50 \mathrm{ft}$ & 72.3 & 77.4 & 77.8 & 77.2 & 5.1 & 5.5 & 4.9 \\
$100 \mathrm{ft}$ & 67.2 & 72.5 & 73.7 & 70.8 & 5.3 & 6.5 & 3.6 \\
$200 \mathrm{ft}$ & 63.4 & 66.8 & 69.1 & 64.4 & 3.4 & 5.7 & 1.0 \\
\hline
\end{tabular}

Table 8-20 shows the absolute noise levels in open space predicted by TNM 2.5 , STAMINA 2.0, and HNP 1.0, as well as their differences between each model and the measured data. For all the receivers $1.5 \mathrm{~m}(5 \mathrm{ft})$ above the ground, three models overpredict the noise levels. HNP slightly out-performed TNM and STAMINA .The error for TNM ranges from $3.4 \mathrm{dBA}$ to $5.3 \mathrm{dBA}$ and $\mathrm{HNP}$ ranges from $1 \mathrm{dBA}$ to $4.9 \mathrm{dBA}$. The over-predictions for STAMINA range from $5.5 \mathrm{dBA}$ to $6.5 \mathrm{dBA}$.

\subsection{Statistical Analysis}

In the previous section, comparisons were made from case to case. A statistical analysis containing the five barrier sites $(01 \mathrm{KY}, 04 \mathrm{CT}, 05 \mathrm{CT}, 08 \mathrm{CA}$, and $11 \mathrm{CA})$ is carried out in this section. All absolute noise levels at the reference at the top of the barriers and two open sites are excluded from the analysis since the insertion loss comparison at each barrier site is the purpose for this study. 
$\square$ Measurement $\square$ TNM $2.5 \square$ STAMINA $2.0 \square$ HNP 1.0

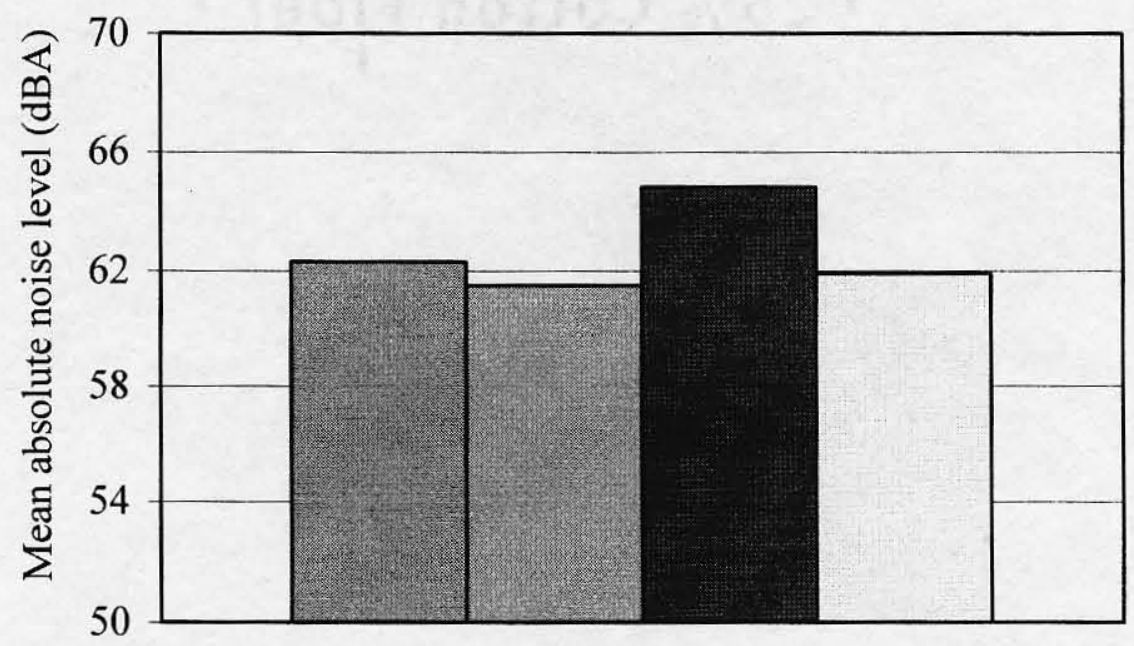

Figure 8-20. Mean absolute noise levels with the barriers

Figure 8-20 shows the comparison of the mean absolute noise levels with the barriers. As can be seen, the predicted noise levels with the barriers for TNM 2.5 and HNP 1.0 are within $1 \mathrm{dBA}$ variations, with $0.8 \mathrm{dBA}$ under-predictions for TNM 2.5 and $0.4 \mathrm{dBA}$ under-predictions for HNP 1.0. STAMINA 2.0 over-predicts the noise levels with the barriers by $2.5 \mathrm{dBA}$. This is may be due to the reason that STAMINA overpredicts the noise levels when receivers are further behind the barrier.

Figure 8-21 shows the comparison of the mean absolute noise levels without the barrier. As can be seen, the predicted noise level for TNM 2.5 is $72.7 \mathrm{dBA}$, versus 72.1 dBA for STAMINA 2.0 and $70.6 \mathrm{dBA}$ for HNP 1.0 in this case.

Figure 8-22 shows the comparison of the insertion loss by TNM 2.5, STAMINA 2.0, and HNP 1.0. As can be seen, the mean predicted insertion loss for TNM 2.5 is 11.2 dBA, versus $7.3 \mathrm{dBA}$ for STAMINA 2.0 and $8.7 \mathrm{dBA}$ for HNP 1.0. Therefore, TNM 2.5 over-predicts the insertion loss by $2.5 \mathrm{dBA}$ compared with HNP 1.0 and by $3.9 \mathrm{dBA}$ compared with STAMINA 2.0 in this study. This result is consistent with the theoretical 
analysis of insertion loss by a point source in Chapter VII and is further validated by the field measurements of insertion loss at the site of $01 \mathrm{KY}$.

\section{$\square$ TNM $2.5 \square$ STAMINA $2.0 \square$ HNP 1.0}

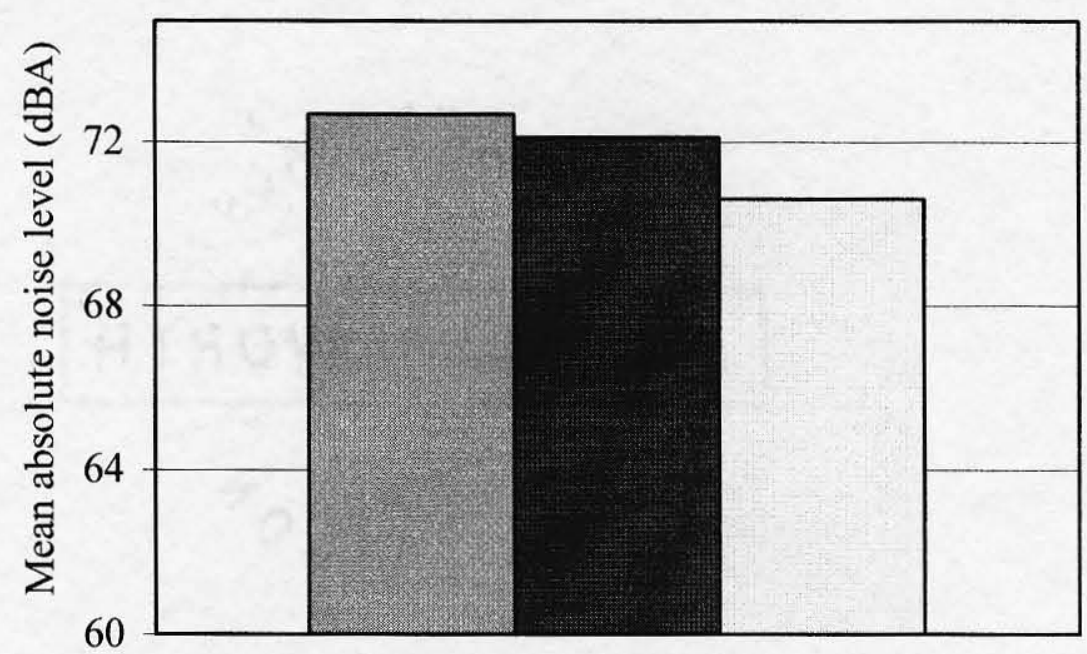

Figure 8-21. Mean absolute noise levels without the barriers

$\square$ TNM 2.5 - STAMINA $2.0 \square$ HNP 1.0

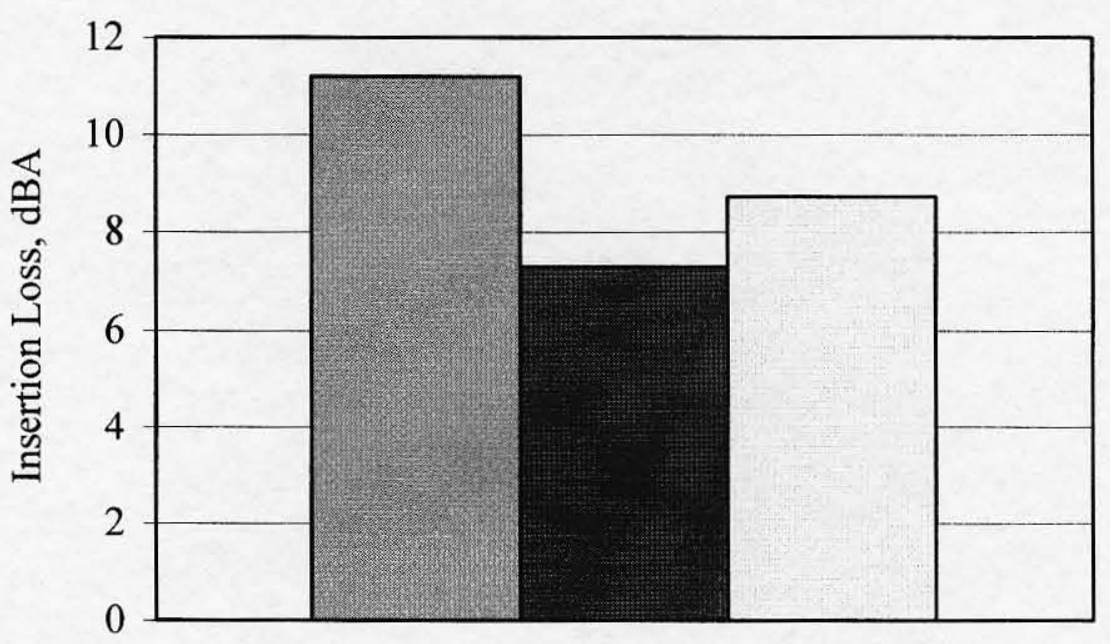

Figure 8-22. Insertion loss comparisons by TNM, STAMINA, and HNP 


\section{CHAPTER IX}

\section{CONCLUSIONS AND RECOMMENDATIONS}

\subsection{Conclusions}

This chapter represents a summary of the findings from this research and a further discussion of the conclusions in the previous chapters. The main goal of this research is to make a comparative study of the physical mechanisms related to highway noise barrier insertion loss, and to evaluate and improve the accuracy of insertion loss of TNM 2.5 compared with STAMINA 2.0, HNP 1.0 and the field measurement data.

To date there have been no independent efforts to examine this issue in great detail. The reasons that TNM 2.5 over-predicts the insertion loss from the perspective of noise diffraction and propagation theories need to be identified, and this proposed research represents the first efforts to do just that. Corrections can be made for more accurate prediction of the insertion loss if it can be proved that the over-prediction of insertion loss by TNM 2.5 is partly caused by the diffraction and propagation theories. Since noise barriers are expensive to build, more accurate prediction of insertion loss by $1 \mathrm{dBA}$, not only means people can be protected by noise barriers with expected satisfaction, but also a significant cost saving potential can be achieved.

Based on the research work in this dissertation, the following findings can be made: 
1. STAMINA 2.0 and HNP 1.0 predict more accurate insertion loss than TNM 2.5 compared with the measurement data.

2. TNM 2.5 over-predicts the insertion loss by about $3 \mathrm{dBA}$ compared with STAMINA 2.0, from the perspective of diffraction theories.

3. TNM 2.5 over-predicts the insertion loss by about $2.5 \mathrm{dBA}$ compared to HNP 1.0 , from the perspective of diffraction and propagation theories. That is to say, an average of $2.5 \mathrm{dBA}$ insertion loss can be improved in TNM 2.5 by the following recommendations. The research makes no attempt to study other reasons that lead to the over-prediction of insertion loss such as sub-source height, source energy splits, and REMEL data.

Based on the comparative study of the physical mechanisms related to highway noise barrier insertion loss, the research shows that the following reasons account for the over-prediction of the insertion loss in TNM 2.5 from the perspective of diffraction and propagation theories. The propagation theories here refer to the reflection theory and impedance discontinuities theory deployed in TNM 2.5.

1. The equations used in TNM 2.5 for the ground wave function, $F(w)$, to calculate the reflection coefficients may lead to inaccuracies when calculating the insertion loss. $F(w)$ is a strictly decreasing function with increasing frequencies. However, the curve of $F(w)$ generated by TNM's method shows a sudden increase at points where the value of $w$ approaches the end point of the asymptotic series, 10. Refer to Chapter IV for details. 
2. $r$, the total distance between the source and the receiver, which is used in TNM 2.5 to calculate $w$, the numerical distance, is not theoretically consistent with the original papers (Chessell, 1977), (Jonasson, 1972). This may lead to inaccuracies when calculating the insertion loss.

3. TNM 2.5 only takes the real source contribution into account when calculating the diffraction coefficients, which may lead to the over-prediction of insertion loss by about 3 dBA compared with STAMINA 2.0, from the perspective of diffraction theories. Refer to Chapter VII for details.

4. TNM 2.5 does not account for the reflection on the receiver side with barriers and only takes two propagation paths to calculate the absolute noise levels with the barriers, which may lead to the over-prediction of insertion loss for receivers close to the barrier and the under-prediction of insertion loss for receivers far from the barrier. Refer to Chapter VII for details.

\subsection{Recommendations}

The following recommendations are proposed based on the above reasons for TNM 2.5.

1. Apply the method by Chien and Soroka (1980) to calculate $F(w)$, the ground wave function. Use the ACM algorithm 680 developed by Poppe (1990) for the complex error function. This method is not only more accurate than the method of TNM 2.5, but also is computationally efficient. According to Chapter IV, a smooth and strictly decreasing curve of $F(w)$ can be obtained by this method. 
2. Substitute $r^{\prime}$, the total distance between the image source and the receiver, for $r$, to calculate $w$, the numerical distance.

3. Take both the real source contribution and image source contribution into account when calculating the diffraction coefficients, which may reduce the insertion loss in TNM 2.5 by about $3 \mathrm{dBA}$ compared with STAMINA 2.0, from the perspective of diffraction theories.

4. Take four propagation paths into account with barrier situations. This model is consistent with the original paper by De Jong (1983), which may improve the accuracy of insertion loss in TNM 2.5 .

5. TNM 2.5 uses an adjustment factor, $A$, to adjust the diffraction field to make it consistent with empirical results, and it is set to 1.2 in TNM 2.5. However, this adjustment is a trade-off since the barrier noise diffraction interacting with the ground reflection is a complex phenomenon, and it is a non-linear system. The introduction of $A$ may lead to additional errors in some cases. By applying Recommendation 3 and Recommendation 4, this adjustment factor may be set back to 1 .

\subsection{Future Research Needs}

Not only this study, but also the previous research work (Harris, 2000), (Barrett, 2001), (Wayson, 2002), (Kim, 2003), provides evidence that TNM 2.5 over-predicts the insertion loss compared to STAMINA 2.0 and the field measurement data. The reasons for this over-prediction have been systematically studied in this research from the perspective of diffraction and reflection theories. Recommendations are made for the 
more accurate calculation of the insertion loss. From one aspect, the research is to study the over-prediction of insertion loss in TNM 2.5. Other aspects such as sub-source height, source energy split, and REMEL data may also lead to the over-prediction of the insertion loss. As a result, future studies may be needed for more accurate predictions of the absolute noise levels and insertion loss in TNM 2.5. 


\section{REFERENCES}

Abramowitz, M. and Stegun, I.A. (1970). Handbook of Mathematical Functions, New York: Dover Publications

Anderson, G., Lee, C., Fleming, G., and Menge, C. (1998). FHWA traffic noise model user's guide, FHWA-PD-96-009, DOT-VNTSC-FHWA-98-1, FHWA, U.S. Department of Transportation.

American National Standards Institute and the Acoustical Society of America Standards, Methods for Determining the Insertion Loss of Outdoor Noise Barriers, ANSI S12.8-1998 (Acoustical Society of America, New York, 1998)

Attenborough, K. (1982). Predicted ground effect for highway noise, Journal of Sound and Vibration 81, p. 413-424.

Barrett, D.E., and Seavey, E.R. (2001). Comparison of STAMINA2.0/OPTIMA to TNM Results and Effects on Noise Barrier Analysis, inter-noise 2001, The Hague, The Netherlands.

Bass, Ronald E., Herson, Albert I. and Bogdan, Kenneth M. (2001). The NEPA BOOK, A step-by-step guide on how to comply with the National Environmental Policy Act, Publishers Press, Salt Lake city.

Barry, T.M. and Regan, J. A. (1978). FHWA Highway Traffic Noise Prediction Model, Report No. FHWA-RD-77-108, Washington, DC: Federal Highway Administration, U.S. Department of Transportation.

Boulanger, P., Waters-Fuller, T., Attenborough, K., and Li, K.M. (1997). Models and measurements of sound propagation from a point source over mixed impedance ground, Journal of the Acoustical Society of America. 102 (3).

Born, M. and Wolf, E. (1965). Principles of Optics, $3^{\text {rd }}$ ed. Pergamon, New York. p. 375382.

Bowlby, W., Higgins, J., and Reagan, J. (1982). Noise Barrier Cost Reduction

Procedures, STAMINA 2.0/OPTIMA User's Manual Report No. FHWA-DP-58-1, Washington, DC: Federal Highway Administration. 
Bowman JJ, Senior TBA. In: Bowman JJ, Senior TBA, Uslenghi PLE, editors. Electromagnetic and Acoustic Scattering by Simple Shapes. Amsterdam: NorthHolland; 1969

Chessell, C. I. (1977). Propagation of noise along a finite impedance boundary, Journal of the Acoustical Society of America 62, p. 825-834.

Chien, C.F., Soroka, W. W. (1980). A note on the calculation of sound propagation along an impedance surface, Journal of Sound and Vibration 69, p. 340-343.

Cohn, L. F. (1980). Bus Noise Levels in the New York City and Albany Metropolitan Areas, Journal of Noise Control Engineering, 14(1), p. 38-41.

Cohn, L. F. and McVoy, G. R. (1982). Environmental Analysis of Transportation Systems, John Wiley \& Sons, Inc., New York.

Cohn, L.F. (1982). Environmental Action Planning for Transportation Project Development, Transportation Quarterly, 36 (4), p. 503-525.

Cohn, L. F. and Bowlby, W. (1984). Minimizing Highway Noise Barrier Intrusion, Journal of Environmental Engineering, 110(3), p. 534-549.

Cohn, L. F. and Harris, R. A. (1987). Environmental Planning in Urban Transportation, Journal of Transportation Engineering, 113 (3) p. 229-247.

Cohn, L. F. and Harris, R. A. (1988). Improving Public Response to Sensitive Transportation Projects, Journal of Transportation Engineering, 114(4), p. 465475.

Cohn, L. F. and Harris, R. A. (2001). Comparing Traffic-Noise Model Accuracy Using State-Specific Emission Data, Journal of Urban Planning and Development, 127(2), p. 79-93.

De Jong, B.A., Moerkerken, A. and Van Der Toorn, J.D. (1983). Propagation of sound over grassland and over an earth barrier, Journal of Sound and Vibration 86(1), p. $23-46$.

Delany, M.E., Bazley, E. N. (1970). Acoustical properties of fibrous absorbent materials, Applied Acoustics 3 p. 105-116.

Delany, M.E., Bazley, E. N. (1971) A note on the effect of ground absorption in the measurement of aircraft noise, Journal of sound and vibration 16, p. 315-322.

Embleton, T. F. W., Piercy, J. E., Olson, N. (1976). Outdoor sound propagation over ground of finite impedance, Journal of the Acoustical Society of America 59, p. 267-277. 
Embleton, T. F. W., Piercy, J. E., and Daigle, G.A. (1983). Effective Flow Resistivity of Ground Surfaces Determined by Acoustical Measurements, Journal of the Acoustical Society of America, Vol. 74, p. 1239-1244.

Federal Highway Administration (FHWA). (1978). Highway Noise Measurements for Verification of Prediction Models, DOT-TSC-OST-78-2/DOT-TSC-FHWA-78-1, January 1978.

Federal Highway Administration (FHWA). (2004). Priority, Market-Ready Technologies and Innovations, FHWA Traffic Noise Model, Version 2.1, http://www.fhwa.dot.gov/rnt4u/ti/trafficnoise.htm

Federal Highway Administration (FHWA). Highway Traffic Noise Barrier Materials and Cost By State, http://www.fhwa.dot.gov/environment/noise/barrier/materials.htm

Federal Highway Administration (FHWA). (1998). Memorandum to FHWA regional offices from the director, Office of Environment and Planning, FHWA-98-2, Washington, D.C.

Federal Highway Administration (FHWA). (2002). Memorandum on Highway Traffic Noise FHWA Traffic Noise Model Validation and Phase-In, FHWA-2002-10, Washington, D.C.

Federal Highway Administration (FHWA). (2004). Memorandum to Division Administrators from the director, Office of Natural and Human Environment, FHWA-2004-8, Washington, D.C.

Fleming, G., Rapoza, A., and Lee, C. (1995). Development of National Reference Energy Mean Emission Levels for the FHWA Traffic Noise Model (FHWA TNM), Version 1.0, Report FHWA-PD-96-008/DOTVNTSC-FHWA-96-2, Cambridge, MA., U.S. Department of Transportation

FHWA, Highway Traffic Noise Barrier Construction Trends - Introduction, www.fhwa.dot.gov/environment/noise/barrier/tintro.pdf

Galloway, W. J., Clark, W. E., and Kerrick, J. S. (1969). Highway Noise Measurement, Simulation, and Mixed Reactions, NCHRP Report 78.

Gordon, C. G., Galloway, W. J., Kugler, B. A., and Nelson, D. L. (1971). Highway Noise-A Design Guide for Highway Engineers, NCHRP Report 117.

Harris, R. A. (1984). Determination of Reference Energy Mean Emission Level in Georgia, Transportation Research Record 983, TRB, National Research Council, Washington, D.C., p. 22-27. 
Harris, R. A. (1985). Development of an Expert System to Control a Highway Noise Barrier Design Model, Ph.D. Dissertation, Department of Civil Engineering, Vanderbilt University, Nashville TN., p. 2.

Harris, R., and Cohn, L. (1999). Case study: TNM vs. STAMINA in Kansas, http://ceetranspo.spd.Louisville.edu/noise/tnmcomp.pdf

Harris, R. A., Cohn, L. F. (2000). Evaluation of the Federal Highway Administration's Traffic Noise Model, Journal of Transportation Engineering, 126(6), p. 513-520.

Harris, R. A. (2004). Traffic Noise Model 2.5 forum, http://www.trafficnoisemodel.org/phpBB204/

Hadden, W.J., Pierce, A.D. (1981). Sound diffraction around screens and wedges for arbitrary point-source locations. Journal of the Acoustical Society of America, 69 (5) p.1266-76.

Hankard Environmental, (2002). Noise Model Comparison Report: Interstate 25 through Colorado Springs Environmental Assessment, Report No. HEI 4-9-1, EI Paso County, Colorado.

Hendriks, R. W. (1985). California Vehicle Noise Emission Levels, Transportation Research Record 1033, TRB, National Research Council, Washington, D.C., p.60-70.

Hendriks, R. W. (1995). Traffic Noise Attenuation as a Function of Ground and Vegetation (Final Report), Report No. FHWA/CA/TL-95/23, California Department of Transportation, Engineering Service Center.

Herman, L., Bowlby, W., T. O'Grady, Chen, C., Jamison, M., Wayson, R. (1991), Determination of Traffic Noise Barrier Effectiveness, Report No. TDOT-I440-1, Vanderbilt Engineering Center for Transportation Operations and Research, Vanderbilt University

Herman, L. A. (1996). An evaluation of STAMINA 2.0 using the ORNAMENT ground attenuation algorithm, Journal of the Acoustical Society of America, 100(5), p. 3460-3463.

Hothersall, D.C. and Harriot, J.B.N. (1995). Approximate models for sound propagation above multi-impedance plane boundaries, Journal of the Acoustical Society of America, 97.

Ingard, U. (1951). On the reflection of a spherical sound wave from an infinite plane, Journal of the Acoustical Society of America 23, p. 329-335. 
Isei, T., Embleton, T.F.W. and Piercy, J.E. (1980). Noise reduction by barrier on finite impedance ground, Journal of the Acoustical Society of America, 67(1).

Jonasson, H. (1971). The propagation of sound over ground with and without acoustic barriers. Report 18, Lund, Sweden.

Jonasson, H.G. (1972). Sound reduction by barriers on the ground, Journal of Sound and Vibration 22, p. 113-126.

Kawai, T., Fujimoto, K., and Itow, T. (1978). Noise propagation around a thin half plane, Acoustica 38, p. 313-323.

Keller J. B. (1962). The geometrical theory of diffraction, J. Opt. Soc. 52 p. 116-30.

Kim, Teak-Keun, Highway Traffic Noise Emission Levels: A Comparison of National Averages to Individual State Data, Ph.D. dissertation, Dept. of Civil Engineering, University of Louisville, Louisville, KY, p18.

Kim, T. K, Harris, R. A, Cohn, L. F., Kim, J. S. (2003). A Comparison of Differences in Highway Noise Barrier Design Between STAMINA and TNM, inter-noise 2003, Sogwipo, Korea.

Kugler, B .A., Commins, D. E., and Galloway, W. J. (1976). Highway Noise - A Design Guide for prediction and Control, NCHRP Report 174.

Kugler, B. A. and Piersol, A. G. (1973). Highway Noise, A Field Evaluation of Traffic Noise Reduction Measures, NCHRP Report 144.

Kurze, U. J, Anderson, G.S. (1971). Sound attenuation by barriers, Applied Acoustics 4 p. 35-53.

Kurze, U.J. (1974). Noise reduction by barriers. Journal of the Acoustical Society of America 55 p. 504-18.

Lee, Cynthia S.Y. (1996). Measurement of Highway-Related Noise, U.S. Department of Transportation Research and Special Programs Administration, John A, Volpe National Transportation Systems Center.

Li, K.M., Wong, H.Y. (2005). A review of commonly used analytical and empirical formulae for predicting sound diffracted by a thin screen, Applied Acoustics 66. $\mathrm{p}$. 45-76.

Maekawa, Z. (1966). Noise Reduction by Screen of Finite Size, Memoirs of the faculty of engineering, Kobe University, No. 12.

Maekawa Z. (1968). Noise Reduction by Screens. Applied Acoustics 1968; 1: p. 157-73 
MacDonald, H. M. (1915). A class of diffraction problems, Proc. Lond. Math. Soc. 14 p. 410-27.

Menge, C. W., Rossano, C. F., Anderson, G. S., and Bajdek, C. J. (1998). FHWA Traffic Noise Model, Version 1.0 Technical Manual, Report No. DOT-VNTSC-FHWA98-2, U.S. Department of Transportation, FHWA.

Menounou, P. (2001). A correction to Maekawa's curve for the insertion loss behind barriers, Journal of the Acoustical Society of America 110 p. 1828-38.

Ögren, M., Propagation of sound-Screening and ground effect Part I: Non-refracting atmosphere. SP REPORT 1997:44

Parkin, P. H., Scholes, W. E. (1964). The horizontal propagation of sound from a jet engine close to the ground, at Radlett, Journal of Sound and Vibration, 1, p. 1-13.

Parkin, P. H., Scholes, W. E. (1965). The horizontal propagation of sound from a jet engine close to the ground, at Hatfield, Journal of Sound and Vibration, 2, p. 353374.

Pierce, A. D. (1974). Diffraction of sound around corners and over wide barriers, Journal of the Acoustical Society of America. 55(5) p. 941-55.

Pierce, A. D. (1989). Acoustics, An introduction to its physical principles and applications, McGraw-Hill Book Company, New York

Poppe, G.P.M. and Wijers, C.M.J. (1990). More Efficient Computation of the complex error function, ACM Transactions on Mathematical Software. Vol. 16, No.1.p. 38-46.

Rasmussen, K.B. (1981). Sound propagation over grass covered ground, Journal of sound and vibration 78(2), p. 247-255.

Rasmussen, K.B. (1981). Sound propagation over an impedance discontinuity, Proceeding of Inter-Noise 81, p. 229-232.

Rasmussen, K.B. (1982). A note on the calculation of sound propagation over impedance jumps and screens, Journal of Sound and Vibration 84(4), p. 598-602.

Redfearn, S.W. (1940). Some acoustical source-observer problem, Philos. Mag. 30 p. 223-36.

Rochat, J. L. and G.G. Fleming. (2002). Validation of FHWA's Traffic Noise Model (TNM): Phase 1, Report No. FHWA-EP-02-031 and DOT-VNTSC-FHWA-02-01. 
Cambridge, MA: John A. Volpe National Transportation Systems Center, Acoustics Facility.

Rochat, Judith L., Fleming, Gregg G. (2004). Addendum to Validation of FHWA's Traffic Noise Model (TNM): Phase 1, U.S. Department of Transportation, Volpe National Transportation Systems Center, Acoustics Facility, Cambridge, MA.

Scholes, W. E., Salvidge, A. C., and Sargent, J.W. (1971). Field Performance of a Noise Barrier, Journal of Sound and Vibration, vol.16, p. 627-642.

Sheffer, D.M. (1994). Verification of Noise Analysis Using STAMINA 2.0, MENG Thesis, Dept, of Civil Engineering, University of Louisville, Louisville, KY, p. 52.

Sisti, Alex F. and Farr, Steven D. (2000). Model Abstraction Techniques: An Intuitive Overview, Air Force Research Laboratory/IFSB.

Sommerfeld, Arnold. (1954). Optics, Lecture on Theoretical Physics, Academic Press Inc., Publishers, New York, N.Y.

Staiano, M. A. Traffic Noise Model vs. Extreme Topography, Transportation Research Record 1859, Paper No. 03-2856, p. 65-71

Thomasson, S. I. (1977). Propagation of noise along a finite impedance boundary, Journal of the Acoustical Society of America 62, p. 825-834.

Thomasson, S. I. (1978). Diffraction by a screen above an impedance boundary, Journal of the Acoustical Society of America 63, p. 1768-1781.

U.S. Department of Transportation (USDOT). (1997). Memorandum to state department of transportation agency representatives. USDOT Volpe Transportation Systems Center, Cambridge, Mass.

Yamamoto, K., Takagi, K. (1992). Expressions of Maekawa's chart for computation, Applied Acoustics 37 p. 75-82.

Wayson, R. L., Ogle, T. W. A., and Lindeman, W. (1993). Development of Reference Energy Mean Emission Levels for Highway Traffic Noise in Florida, Transportation Research Record 1416, TRB, National Research Council, Washington, D.C., p. 82-91.

Wayson, R. L., MacDonald, J. M., A. El-Aassar, W. Arner, (2002). Continued Evaluation of Noise Barriers in Florida, Final Draft Report, Report No. FL-ER-85-02, University of Central Florida, Community Noise Lab: Civil \& Environmental Engineering, Orlando, Florida. 
Wayson. R, MacDonald, J., A. El-Aassar, Linder, W., and Berrios, M., Florida Noise Barrier Evaluation and Computer Model Validation, Transportation Research Record 1859, Paper No. 03-3296, p. 72-78.

Wayson, R. L., MacDonald, J. M., A. El-Aassar, and Chua, C. B. (2003) Comprehensive Review of Collected Noise Information at Barrier Sites, Final Report, Report No. BC355/RPW07, University of Central Florida, Community Noise Lab: Civil \& Environmental Engineering, Orlando, Florida.

Weideman, J.A.C. (1994). Computation of the Complex Error Function, SIAM Journal on Numerical Analysis, Vol.31, No. 5, p. 1497-1518. 


\section{APPENDIX I}

Calculation of $F(w)$ based on TNM's method, ACM 680 Algorithm and FFT method 
Table 4-2. TNM's method to calculate $F(w)$

\begin{tabular}{|c|c|c|c|c|}
\hline $\begin{array}{c}\text { Froptency, } \\
H z\end{array}$ & w & abs $\{\mathbf{w}\}$ & {$[\because(k)$} & $\operatorname{abs}(\mathbf{F}(w)$ \\
\hline 60 & 5. $48257352179857 \mathrm{E}-602+8.85889525992292 \mathrm{E}-002 \mathrm{i}$ & 0.104182 & $0.663788125457692+0.330275581766596 \mathrm{i}$ & 0.741415293 \\
\hline 63 & $8.510100702177640-002+0.1183349252746141$ & 0.14576 & $0.606090004025928+0.372325803377441 \mathrm{i}$ & 0.711316806 \\
\hline 80 & $0.134723418118063+0.16048039810124 \mathrm{i}$ & 0.209534 & $0.53134282961321 \div 0,41643983825735$ & 0.675090617 \\
\hline 200 & $0.207887060964095+0.214386790071051 i$ & 0.298628 & $0.444399802509565+0.453701544753503 \mathrm{i}$ & 0.635087613 \\
\hline 125 & $0.322303744909504+0.2878138233537221$ & 0. 132127 & 0. $33891545137062+0.479629809544575 \mathrm{i}$ & 0.587289058 \\
\hline 160 & $0.626481816867112+0.4012753248071241$ & 0.66197 & $0.202869751194919-0.481838663434354 \mathrm{i}$ & 0.522804584 \\
\hline 200 & $0.82455410625467+0.54533064426145 \mathrm{i}$ & 0.988572 & 7. $3157407368825 \mathrm{E}-002+0.446113950038604 \mathrm{i}$ & 0.452077564 \\
\hline 250 & 1. $29735800829688+0.7466054692657981$ & 1.496819 & $-4.1002766313736 \mathrm{E}-002+0.3671888897361421$ & 0.369471119 \\
\hline 315 & 2. $08392683480152-1.04408874253509 \mathrm{i}$ & 2.330848 & $0.113498303297007+0.251265779568938 \mathrm{i}$ & 0.27571064 \\
\hline 460 & $3.4134169131053-1.49789642512844$ & $3.72941 \%$ & $-0.129688695285673+0.136151638970583 \mathrm{i}$ & 0.188033046 \\
\hline 500 & $5.4348248739014+2.135735960686661$ & 5.83909 & $0.128224994433790+0.160519253446851 i$ & 0.205446051 \\
\hline 630 & $8.8084+494145879+3.15721059221847$ & 9.357192 & $0.35041813254823+0.555129432178736 i$ & 0.65647662 \\
\hline 800 & $11.5179386738325+1.87804367224312$ & 15.31554 & $-3.38196174217504 \mathrm{E}-002+1.29097781240907 \mathrm{~F}-002 \mathrm{i}$ & 0.036199847 \\
\hline 1000 & $23.121693045673+7,50515314854838$ & 24.32785 & $-2.06372412766833 \mathrm{E}-002-7.2673766515559 \mathrm{E}-003$ & 0.021879454 \\
\hline 1250 & $36.7019729037725+2.1151240563899$ & 38.64085 & $-1.276765220: 287 \mathrm{E}-002-4,38369108936859 \mathrm{E}-003$ & 0.013442315 \\
\hline 1600 & $60.784+977388451+21.0935478624281$ & 64.34045 & $7.18777282291388 \mathrm{E}-003+2.6652764757325 \mathrm{E}-003 \mathrm{i}$ & 0.007948319 \\
\hline 200 & $95.059065341104+35.6310586818031$ & 101.5175 & $-1.6676248876314 E-003+1.778166391728841-003$ & 0. 004994857 \\
\hline 260 & $176.978008867961 \cdot 60.95208969682$ & 159.1155 & $-2.92377800078138 \mathrm{E}-003+1.2251473207881 \mathrm{~L}-003 \mathrm{~L}$ & 0.003170089 \\
\hline 8130 & $227.338994949523+106.627590270067$ & 251.1024 & $-1.81041135677347 \mathrm{E} 003+8.5409210654962 \mathrm{~L}-004 \mathrm{~L}$ & 0.002002071 \\
\hline$\$ 000$ & $349.865256602865-188.80499173844$ & 897.6388 & $-1.10940+32577136-003+6.012801339632716-6011$ & 0.00126187 \\
\hline 800 & 512. 4034162954+317.515\%1813235\% & 602.8017 & $-7.0598223765849 E-004+4.38756719879494 \mathrm{E}-0045$ & 0.000831215 \\
\hline 800 & $72.60979927533+573.189495982561 \mathrm{i}$ & $914.6 \times 3$ & $-4.445867564987228-004+3.9988371759721 \mathrm{E}-0011$ & 0.000547675 \\
\hline $86(16)$ & $1059.75140 \times 01584-887.6 \times 9904974881 i$ & 1382.121 & 2.733350276047792-004+2, 326342907781385-004i & 0.000361985 \\
\hline $\ln 00$ & 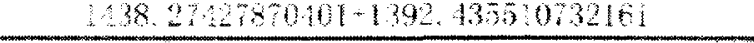 & 2001.87 & $-1.70453244541145004+1.739155070751390-0041$ & $0.0002 \div 99$ \\
\hline
\end{tabular}


Table 4-3. ACM 680 Algorithm to calculate $F(w)$

\begin{tabular}{|c|c|c|c|c|}
\hline $\begin{array}{c}\text { requency, } \\
\mathrm{Hz}_{\mathrm{z}}\end{array}$ & w & $\operatorname{abs}(w)$ & $F(w)$ & $\operatorname{abs}(f(x))$ \\
\hline 50 & 5. $18257352179857 \mathrm{E}-002+8.85889525992292 \mathrm{E}-002 \mathrm{i}$ & 0.104182 & $0.852763044458277+7.96226946526699 \mathrm{E}-002 \mathrm{i}$ & 0.856472173 \\
\hline 63 & $8.51040070247764 \mathrm{~F}-002+0.118334925274614 \mathrm{i}$ & 0.14576 & $0.804972225242369-0.115444966572218 \mathrm{i}$ & 0. 13205352 \\
\hline 80 & $0.134723418118063+0.160480598101241$ & 0.209534 & $0.736822750683943-0.165459135176267 i$ & 0.755171829 \\
\hline 100 & $0.207887060964095+0.214386790071051 i$ & 0.298628 & $0.648248797684602+0.223387525719786 \mathrm{i}$ & 0.685659165 \\
\hline 125 & $0.322303749909504+0.287843823353722 i$ & 0.432127 & $0.526147487473502+0.2844436562295181$ & 0.598113177 \\
\hline 160 & $0.526481816867142+0.4012753248071241$ & 0.661971 & $0.345271583137946+0.329348271385625 \mathrm{i}$ & 0.477161136 \\
\hline 200 & $0.824554110625467+0.545330644261451$ & 0.988572 & $0.158739375064415+0.30931263440033 i$ & 0.347667219 \\
\hline 250 & $L_{\times} 29735800829688+0.746605469265798 i$ & 1. 496849 & $1.3012552400482 \mathrm{E} 002+0.212659352882863 i$ & 0.213057037 \\
\hline 315 & $2.08392683480152-1.04407874253509 i$ & 2.330848 & $3.56122647326 \mathrm{E}-002+9.42558834336952 \mathrm{E}-002 \mathrm{~L}$ & 0.100759143 \\
\hline 400 & $3.41541779731055+1,49789642512184 i$ & 3. 729447 & $--2.329610723465 \mathrm{E}-002+3.0640160688963 \mathrm{E} \cdots 002 \mathrm{i}$ & 0.038490623 \\
\hline 500 & $5.4344824873901 \times 2.13573596068666 \hat{i}$ & 5.83909 & $-1.075315585445 \mathrm{E}-002+1.0665066421777 \mathrm{E}-0025$ & 0.015145098 \\
\hline 630 & $8.80846494145879+3.157210592218471$ & 9.357192 & $-4.42758991660996 \mathrm{E}-003+3.72611246102506 \mathrm{E}-003 \mathrm{i}$ & 0.005786965 \\
\hline 800 & $14.5179386798325+4.87804367224312$ & 15.31554 & $-1.70275776100004 F-003+1.30038425118528 \mathrm{E}-003 \mathrm{i}$ & 0.002142518 \\
\hline 1000 & $23.1216930457775+7.56515314854838$ & 24.32785 & $-6.82070693369896 \mathrm{E}-004+5.014203878001 \mathrm{E}-0041$ & 0.000446548 \\
\hline 1250 & $36.7019729037725+12.1151240563899 \mathrm{i}$ & 38.64985 & $-2.69036495730068 \mathrm{E}-004+1.99584736286396 \mathrm{E}-004 i$ & 1). 000334985 \\
\hline 1600 & $60.7844977388451+21.09354786242811$ & 64.34045 & $9.48285863200993 \mathrm{E}-005 \cdot 7.48606087242476 \mathrm{E}-005 \mathrm{I}$ & 0.000120816 \\
\hline 2000 & $95.059065341104+35.631058681803 i$ & 101.5175 & $-3.65638612100927 \mathrm{E}-005+3.18973260278743 \mathrm{E}-00 \mathrm{i}$ & $4.85217 E-05$ \\
\hline 2500 & $146.978208867961+60.95208969682 i$ & 159.1155 & $-1.39530065099702 \mathrm{E}-005+1.3977406520132 \mathrm{~K}-005 \mathrm{i}$ & $1.97498 \mathrm{E} \cdots 05$ \\
\hline 3150 & $227.338934939523+106.627590270067 \mathrm{i}$ & 251.1024 & $-5.0700747001942 \mathrm{E}-006-6.0975 \mathrm{~L} 54589376 \mathrm{c}$.061 & $7.93003 \mathrm{z}-06$ \\
\hline 4000 & $349.865256602865 \cdot 188.804991754841$ & 397.5688 & 1.73649207990678E $006+2.6443150831223540061$ & $3 \times 163512-06$ \\
\hline 5000 & $512.403451629594+317.515718152353 i$ & 602.8047 & $-6.12465030023435 \mathrm{E}-007+1.23217473502191 \mathrm{E}-006 \mathrm{~J}$ & $1.376 \mathrm{E}-06$ \\
\hline 6300 & $742.608779727513-533.139495982561 \mathrm{i}$ & 914.1693 & $-1.91314039943435 \mathrm{E}-0075.66894904074093 \mathrm{E} 007$ & $3.98297 \mathrm{E}-07$ \\
\hline 8000 & $1060.76140801584-887.689904971381 i$ & 1382.421 & $-4.58756799215365 \mathrm{z}-008+2.5757772731394 \mathrm{E}-007 \mathrm{i}$ & $2.6163 \mathrm{HE}$ \\
\hline 10000 & $1438.27427870401+1392 \times 435510732161$ & $2001.87 i$ & $-4.03964994788453 \mathrm{E} 009 \cdot 1.2470035426481 \% \mathrm{E}-007 \mathrm{I}$ & 1. $24766 \mathrm{~L}-07$ \\
\hline
\end{tabular}


Table 4-4. FFT method to calculate $F(w)$

\begin{tabular}{|c|c|c|c|c|}
\hline $\begin{array}{c}\text { Frequency, } \\
\mathrm{Hz}\end{array}$ & 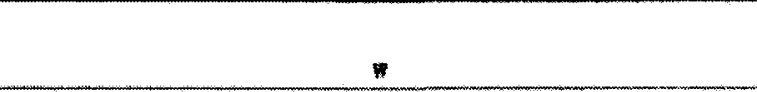 & $a b s(w)$ & $F(w)$ & $\operatorname{abs}(\mathrm{F}(\omega))$ \\
\hline 50 & $5.48257352179857 E-002+8.85889525992292 E-002 i$ & 0.104182 & $0.852761436677106-7,96228983549337 \mathrm{E} \sim 002$ & 0.856470591 \\
\hline 63 & 8. $51040070247764 \mathrm{E}-002+0.118334925274614 \mathrm{i}$ & 0.14576 & $0.804970428028673+0.115444890971558 \mathrm{i}$ & 0.813206562 \\
\hline 80 & $0.134723418118063+0.16048059810124 \mathrm{i}$ & 0.209534 & $0.736821004005341+0.165458482952649 i$ & 0.755169982 \\
\hline 100 & $0.207887060964095+0.214386790071051 \mathrm{i}$ & 0.298628 & $0.64824765775475+0.223386191975657 i$ & 0.685657653 \\
\hline 125 & $0.322303749909504+0.287843823353722 \mathrm{i}$ & 0.432127 & $0.526147675321544-0,284442210620591 \mathrm{i}$ & 0.598112655 \\
\hline 160 & $0.526481816867142+0.4012753248071241$ & 0.661971 & $0.345272569420954-0.329348378888002$ & 0.477161924 \\
\hline 200 & $0.824554110625467+0.54533064426145 i$ & 0.988572 & $0.158738927577642+0.309313044304925 i$ & 0.347667379 \\
\hline 250 & 1. $29735800829688+0.746605469265798 \mathrm{i}$ & 1. 496849 & 1. $3011912839624 \mathrm{E}-002+0.212659204378597 \mathrm{i}$ & 0.21305691 \\
\hline 315 & $2.08392683480152+1.04407874253509 \mathrm{i}$ & 2.330848 & $-3.561234934136 E-002-9.42554782111562 E-002 i$ & 0.100758794 \\
\hline 400 & $3.41541779731055+1.49789642512184 i$ & 3.729447 & $-2.32969670671801 \mathrm{E}-002+3.06396508861986 \mathrm{E}-0021$ & 0.038490738 \\
\hline 500 & 5. $43448248739014+2.13573596068666$ & 5.83909 & $-1.07534683546799 \mathrm{E}-002-1.06678756606858 \mathrm{E}-002 \mathrm{i}$ & 0.015147299 \\
\hline 630 & $8.80846494145879+3.15721059221847 i$ & 9.357192 & $4.42667265692998 \mathrm{E}-003-3.72029119918821 \mathrm{E}-0031$ & 0.005782387 \\
\hline 800 & $14,5179386798325+4.87804367224312 i$ & 15.31554 & $-1.69722074921008 \mathrm{E}-003+1.30627728745192 \mathrm{E}-003 \mathrm{i}$ & 0.002141709 \\
\hline 1000 & $23.1216930457775+7.565153148548381$ & 24.32785 & $-6.86324516619985 \mathrm{E}-004+5.08162305029314 \mathrm{E}-0041$ & 0.000853973 \\
\hline 1250 & 36. $70: 9729037725+12.1151240563899 \mathrm{i}$ & 38.64985 & $-2.75495980259999 \mathrm{E}-004+2.00714412901981 \mathrm{E}-004 \mathrm{i}$ & 0.000340858 \\
\hline 600 & $60.7844977388451+21.0935478624281 \mathrm{i}$ & 64.34045 & $9.91755149100104 \mathrm{E}-005+7.31017046406435 \mathrm{E}-005 \mathrm{i}$ & 0.000123206 \\
\hline 2000 & $95.059065341104+35.631058681803 i$ & 101.5175 & 3. 91121052198962E-005+2. 9850786235297E-005i & 4. $92019 \mathrm{E}-05$ \\
\hline 2500 & $146.978208867961+60.952089696821$ & 159.1156 & 1. $34313508998859[-005+1.23262184664741 E-005 i$ & $1.975 E-05$ \\
\hline 3150 & $227.338934939523+106.627590270067 \mathrm{i}$ & $251 \cdot 024$ & $-5.95371027989344 \mathrm{E}-006+4.93016197289364 \mathrm{E}-006$ & $7.73002 \mathrm{E}-06$ \\
\hline 4000 & $349.865256602865+188.80499175484 \mathrm{i}$ & 397.5588 & $-2.2921529099218 \mathrm{E}-006+1.87156799241752-0061$ & 2.95918E-06 \\
\hline 5000 & $512.403451629594+317.515718152355 i$ & $602.804 \pi$ & $9.92659529908124 \mathrm{E}-007+7.17936846805 \pi 67 \mathrm{~F}-007 \mathrm{I}$ & 1. $23507 \mathrm{E} 06$ \\
\hline 6300 & $742.608779727513+533.139495982561 \mathrm{i}$ & 914.1693 & $4.58222539911901 \mathrm{E} 007+2.32921434084599 \mathrm{E}-007 \mathrm{I}$ & 5. $14024 \mathrm{E}-07$ \\
\hline 8000 & 1059.76140801584+887.689904974381i & 1382.421 & $-2.36203200110197 \mathrm{E}-007-4.46765048844888 \mathrm{E} 008 \mathrm{i}$ & $2.40391 \mathrm{E}$ o? \\
\hline 10000 & $1438.27427870401+1392,436510732161$ & 2001.877 & -1.450875100061928 oot $1.504: 5634947443 E-0081$ & 1. $45865 \mathrm{E}-07$ \\
\hline
\end{tabular}


APPENDIX II

Interface of Highway Noise Predictor 1.0 (HNP 1.0) 


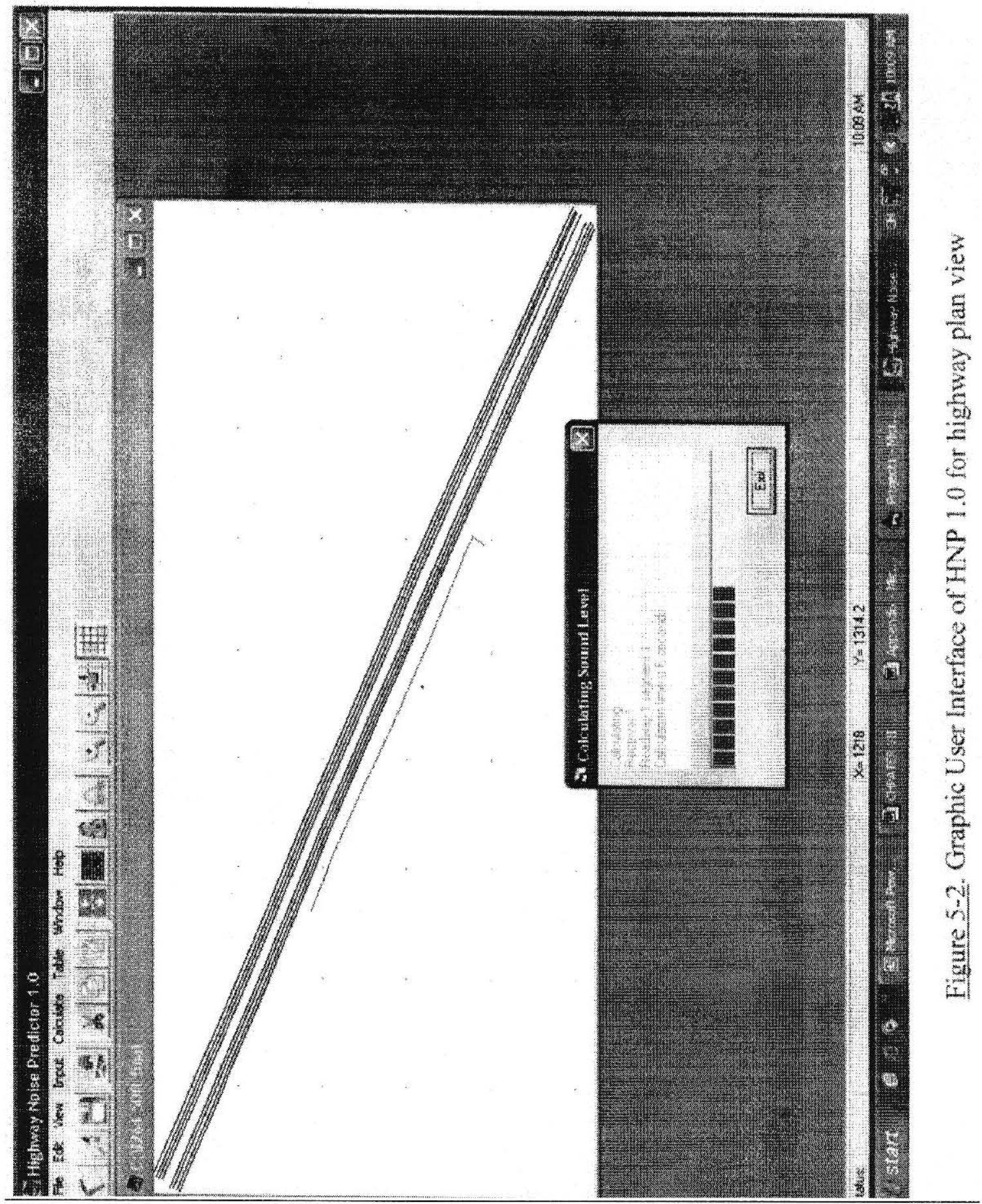




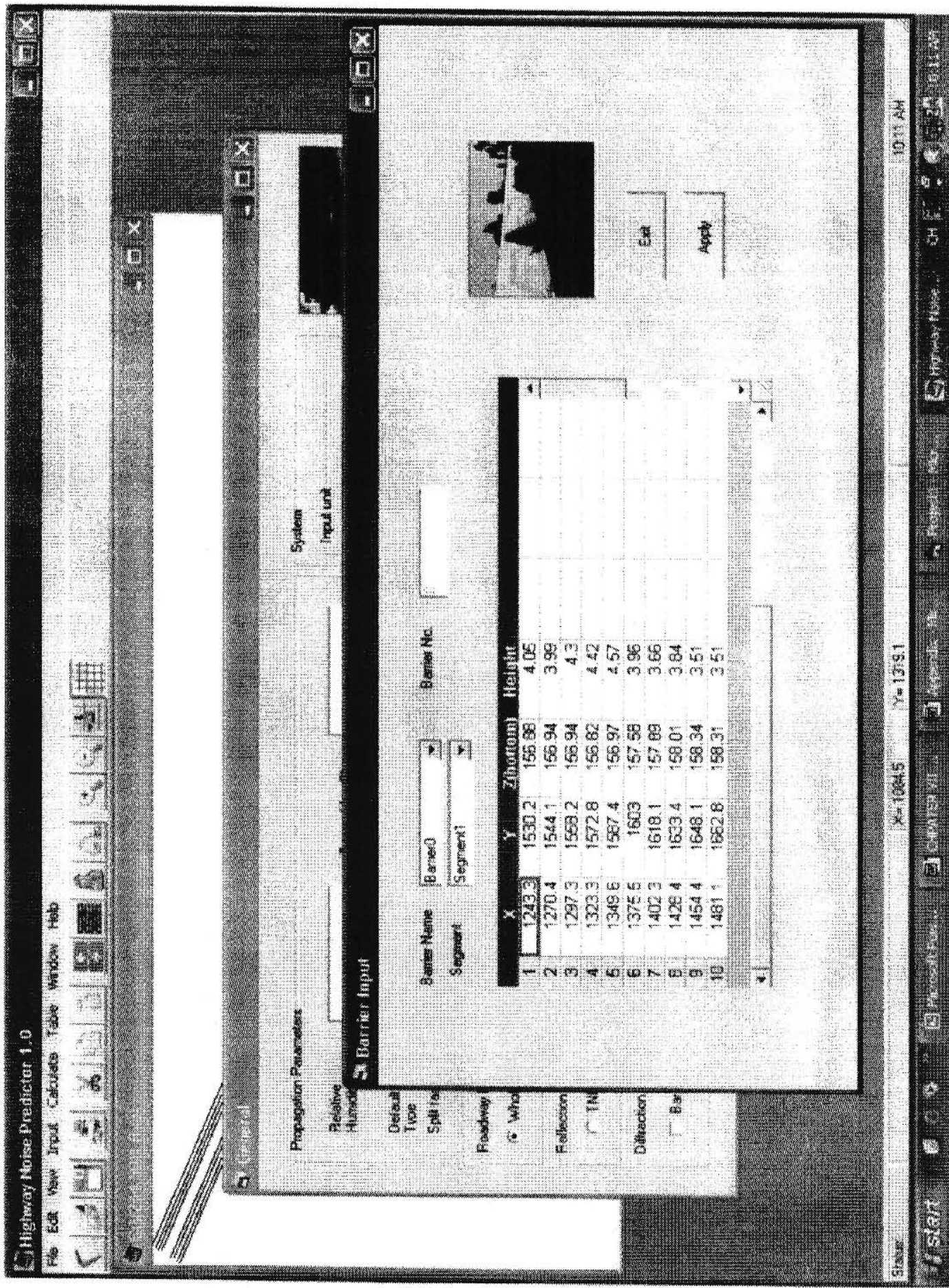

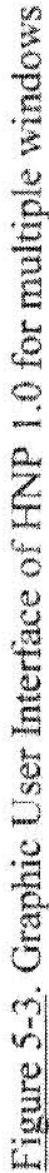


APPENDIX III

Measurement Site Details 

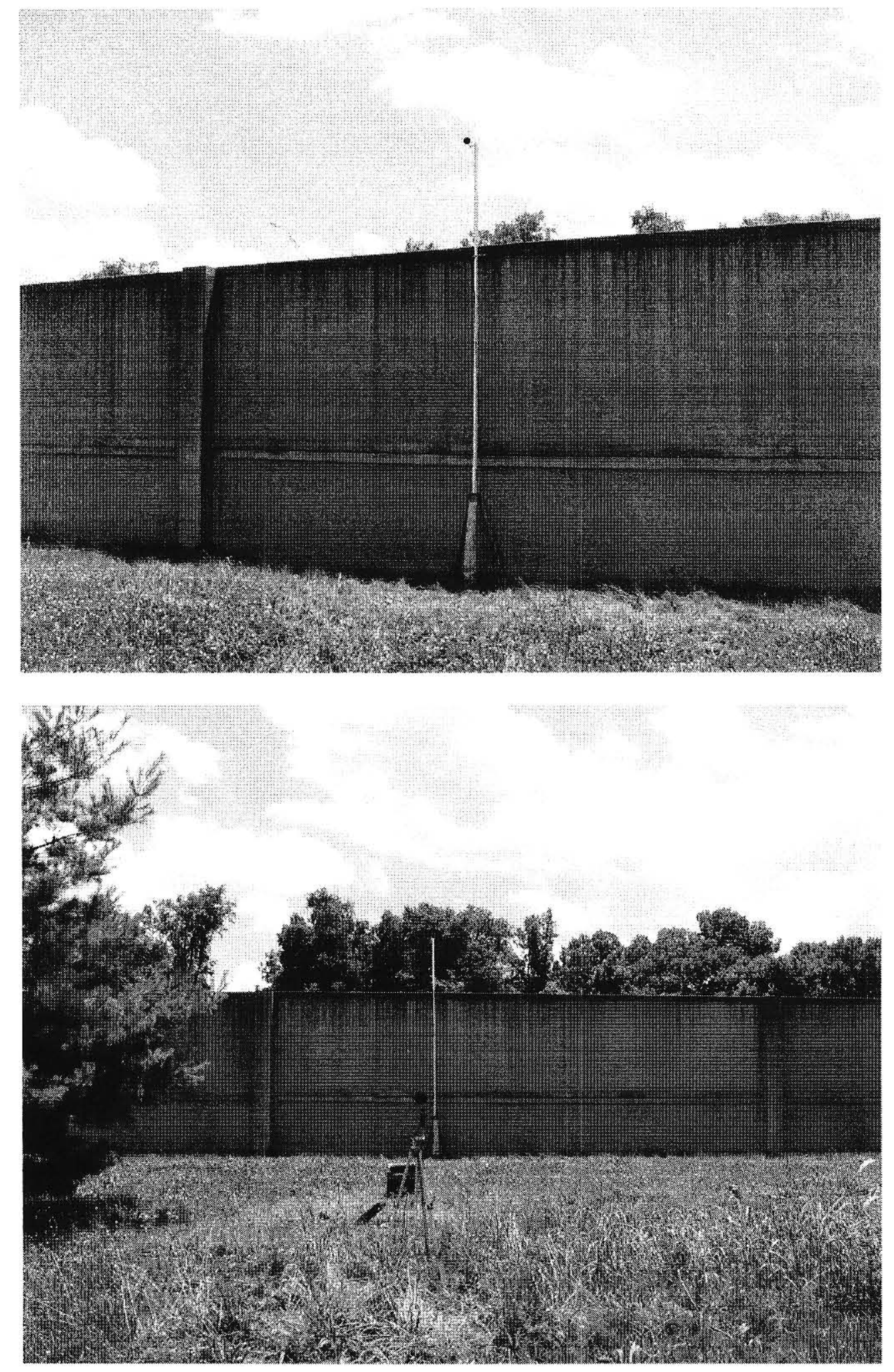

Figure 6-1. Photograph for the field measurements at the barrier site $01 \mathrm{KY}$ 

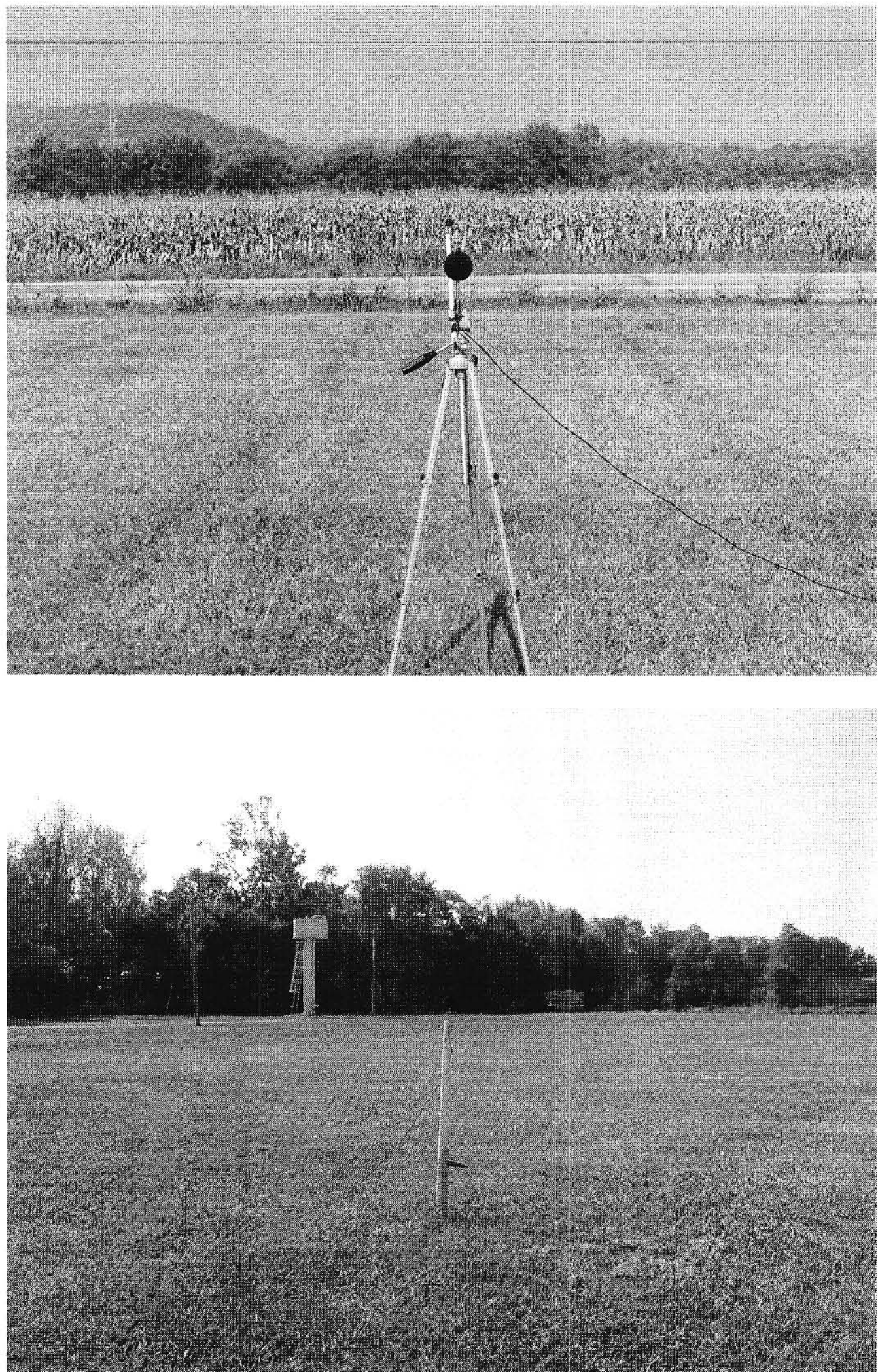

Figure 6-2. Photograph for the field measurements at the open site 04IN 


\begin{tabular}{|l|l|}
\hline Site ID & $01 \mathrm{KY}$ \\
\hline Location & Near Exit 18 of Interstate 264 \\
\hline Site Type & Barrier $(12.6 \mathrm{ft}$ concrete wall), flat \\
\hline Ground Type & Lawn \\
\hline Roadway & 8 lanes, PCC, shoulders, pavement median \\
\hline Instrumentation & Microphones \\
Positions & $\mathrm{bb}=0 \mathrm{ft}$, height $=5 \mathrm{ft}$ above barrier \\
& $\mathrm{bb}=50 \mathrm{ft}$, height $=5 \mathrm{ft}$ \\
& $\mathrm{bb}=100 \mathrm{ft}$, height $=5 \mathrm{ft}$ \\
& $\mathrm{bb}=200 \mathrm{ft}$, height $=5 \mathrm{ft}$ \\
& $\mathrm{bb}=400 \mathrm{ft}$, height $=5 \mathrm{ft}$ \\
& Meteorological Systems \\
& $\mathrm{bb}=50 \mathrm{ft}$, height=6 ft \\
& $\mathrm{bb}=100 \mathrm{ft}$, height $=6 \mathrm{ft}$ \\
\hline
\end{tabular}

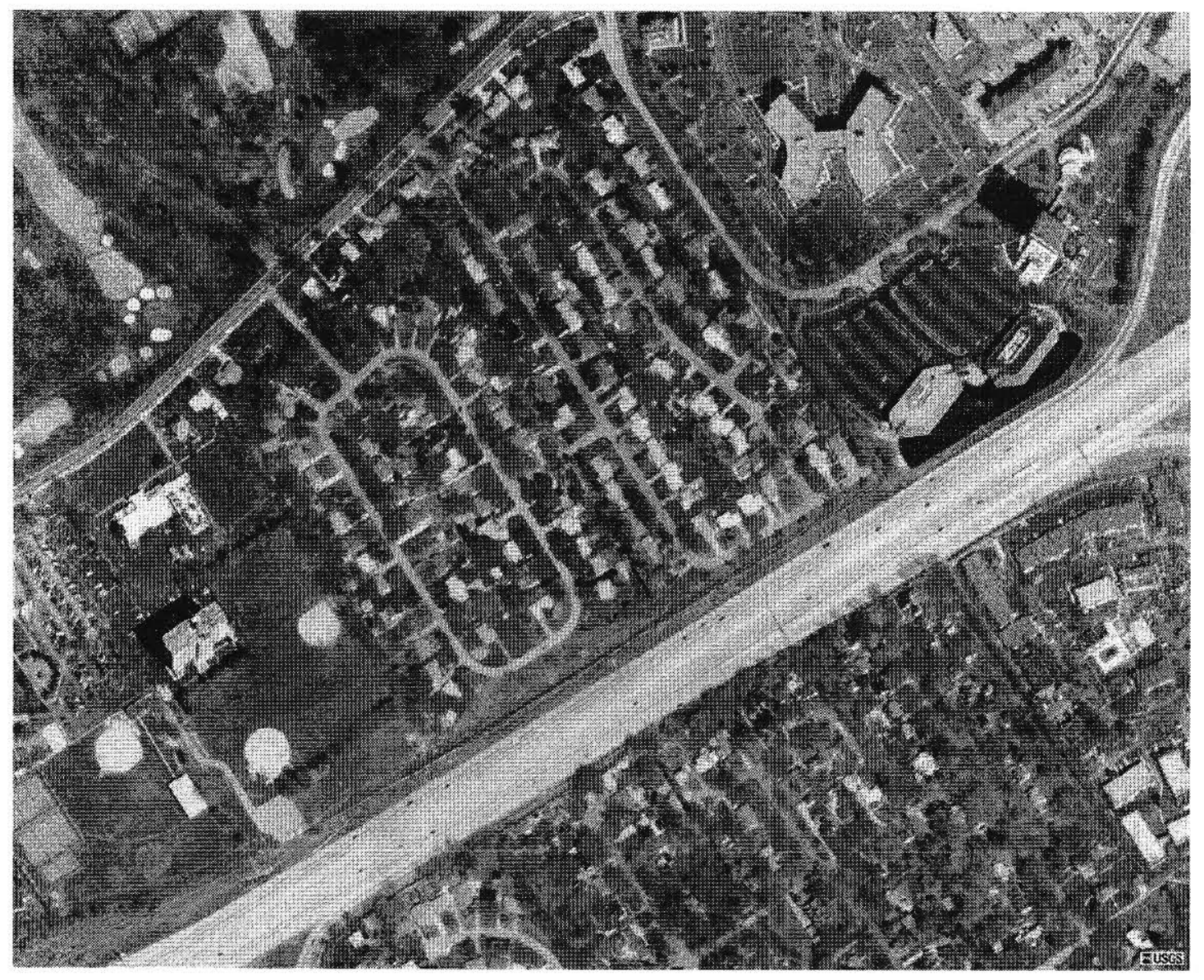

Figure 8-1. Site 01KY: Description and Photograph 


\begin{tabular}{|l|l|}
\hline Site ID & 01KY-TNM model (TNM Version 2.5) \\
\hline Default Ground Type & Lawn \\
\hline Pavement Type & Average \\
\hline TNM objects & Barrier, roadways, receivers, terrain line, \\
\hline
\end{tabular}

Plan view

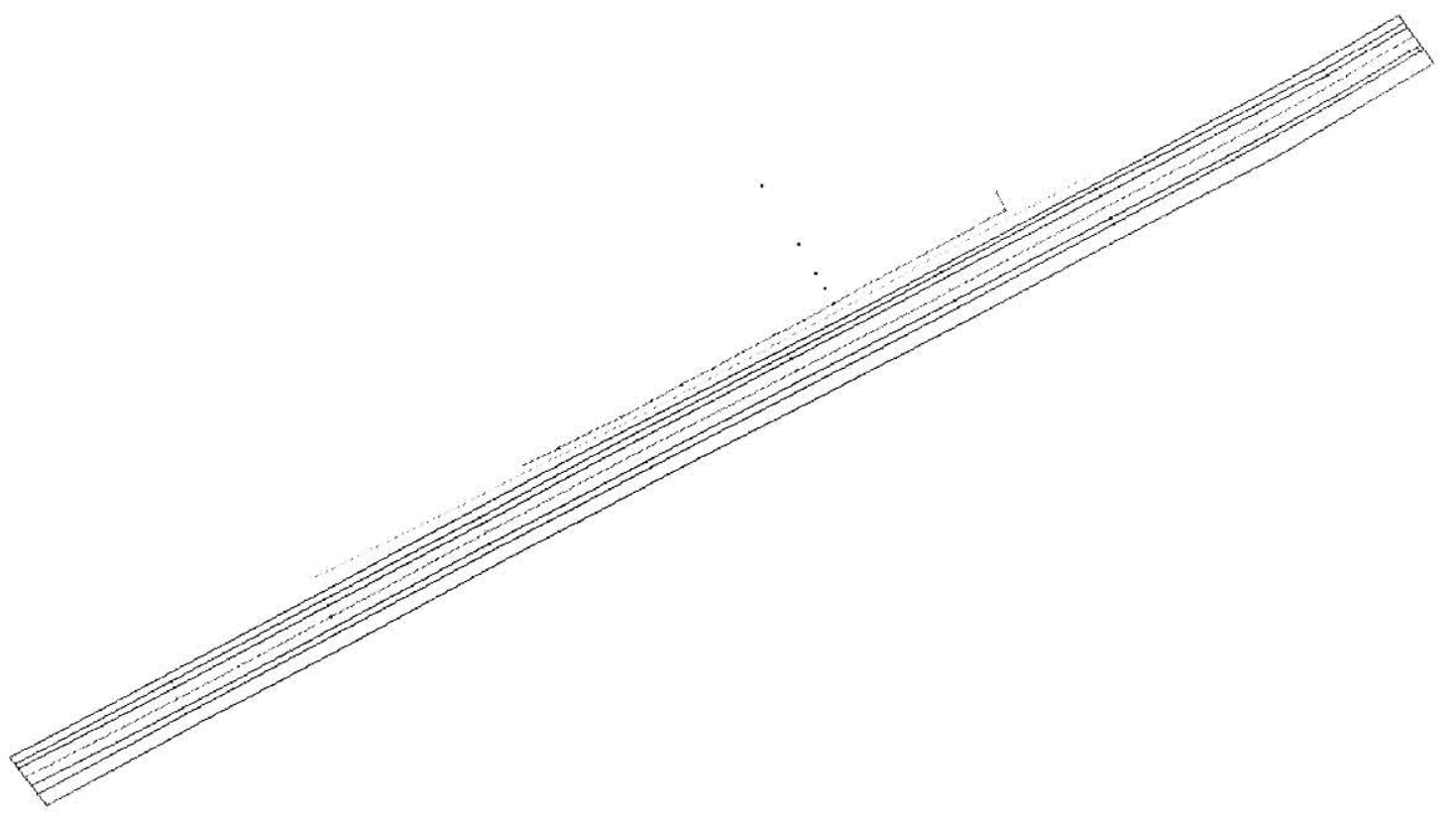

Skew view

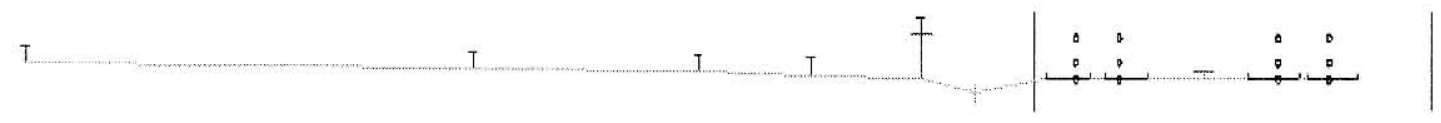

Figure 8-2. Site 01KY: TNM 2.5 Model Description, TNM Plan, Skew View 


\begin{tabular}{|c|c|}
\hline Site ID & $02 \mathrm{KY}$ \\
\hline Location & Near Exit 17 of Interstate 264 \\
\hline Site Type & Barrier (17.0 ft concrete wall), flat \\
\hline Ground Type & Lawn \\
\hline Roadway & 8 lanes, PCC, shoulders, pavement median \\
\hline $\begin{array}{l}\text { Instrumentation } \\
\text { Positions }\end{array}$ & $\begin{array}{l}\text { Microphones } \\
\mathrm{bb}=0 \mathrm{ft} \text {, height }=5 \mathrm{ft} \text { above barrier } \\
\mathrm{bb}=50 \mathrm{ft} \text {, height }=5 \mathrm{ft} \\
\mathrm{bb}=100 \mathrm{ft} \text {, height }=5 \mathrm{ft} \\
\\
\text { Meteorological Systems } \\
\mathrm{bb}=50 \mathrm{ft} \text {, height }=6 \mathrm{ft} \\
\mathrm{bb}=100 \mathrm{ft} \text {, height }=6 \mathrm{ft}\end{array}$ \\
\hline
\end{tabular}

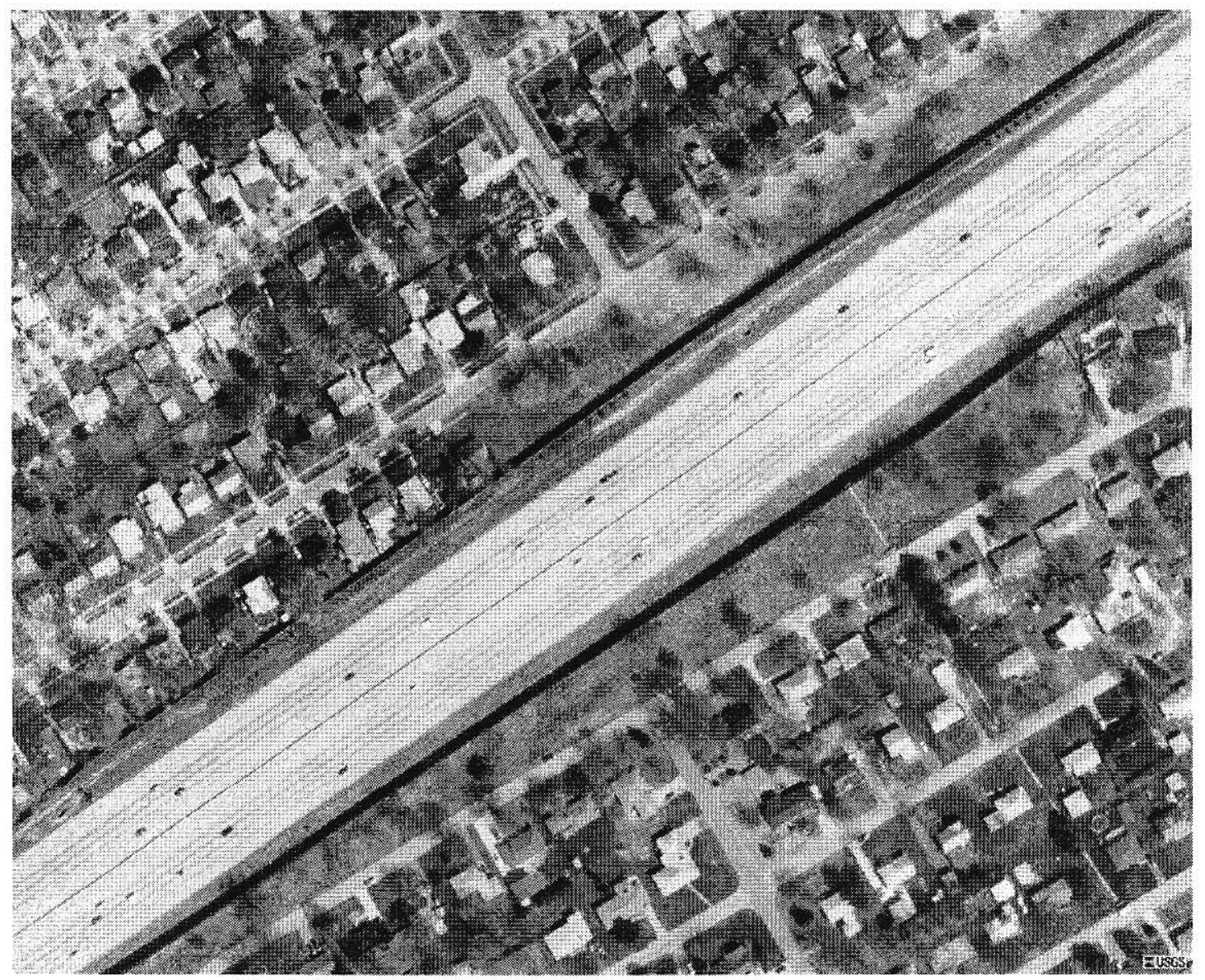

Figure 8-3. Site 02KY: Description and Photograph 


\begin{tabular}{|l|l|}
\hline Site ID & 02KY-TNM model (TNM Version 2.5) \\
\hline Default Ground Type & Lawn \\
\hline Pavement Type & Average \\
\hline TNM objects & Barrier, roadways, receivers, ground zone \\
\hline
\end{tabular}

Plan view

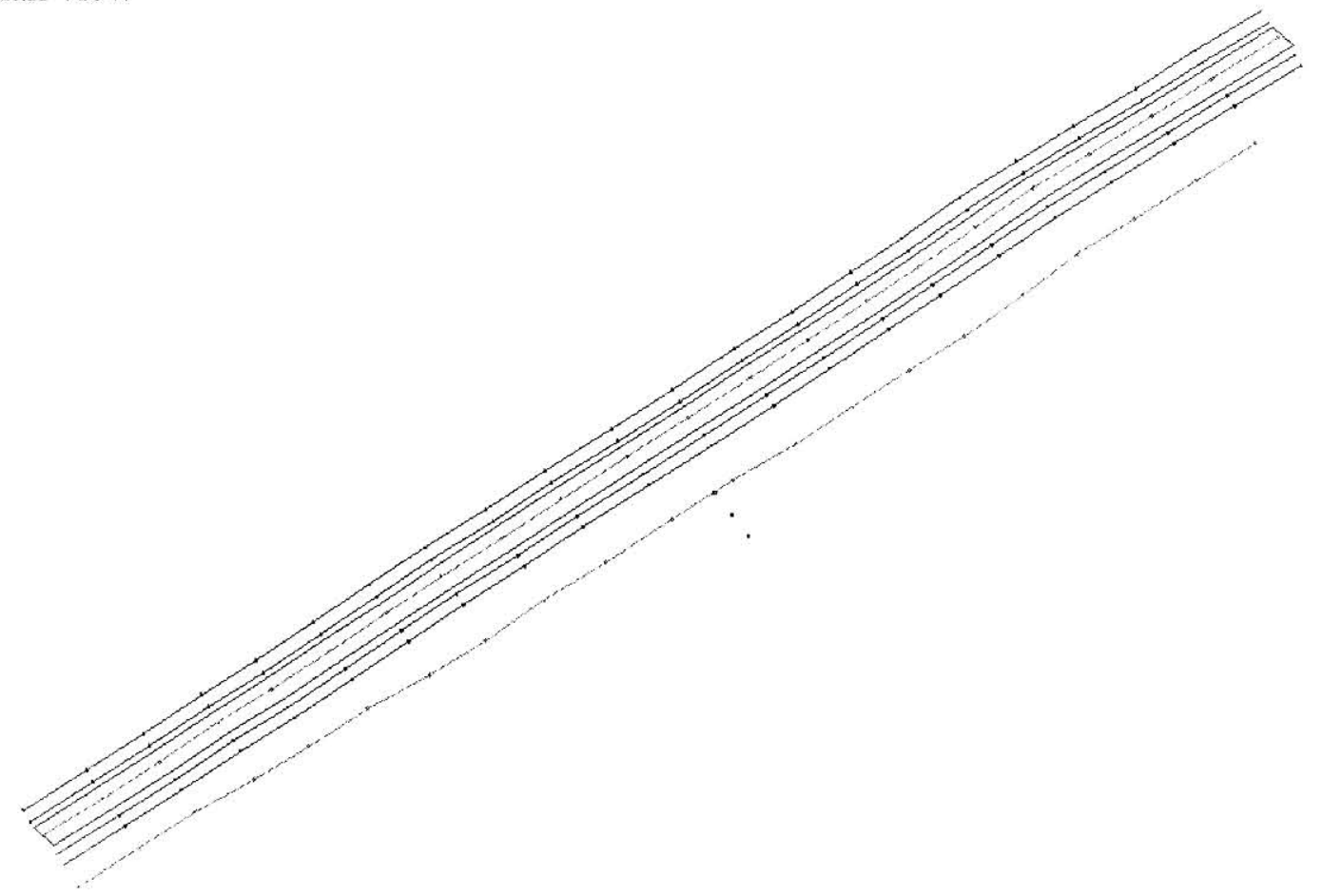

Skew view

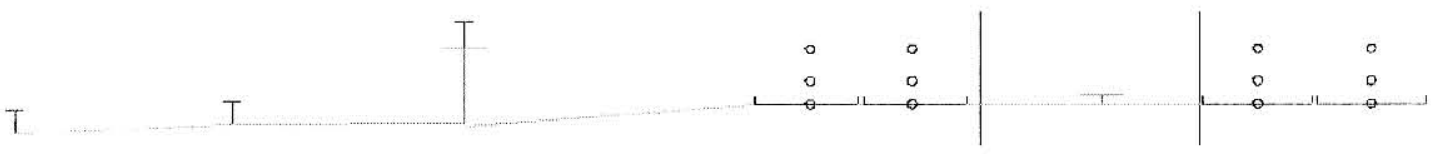

Figure 8-4. Site 02KY: TNM 2.5 Model Description, TNM Plan, Skew View 


\begin{tabular}{|l|l|}
\hline Site ID & 03KY \\
\hline Location & Near Exit 18 of Interstate 264 \\
\hline Site Type & Open, flat space \\
\hline Ground Type & Lawn \\
\hline Roadway & 8 lanes, PCC, shoulders, pavement median \\
\hline Instrumentation & Microphones \\
Positions & $\mathrm{bb}=50 \mathrm{ft}$, height $=5 \mathrm{ft}$ \\
& $\mathrm{bb}=100 \mathrm{ft}$, height $=5 \mathrm{ft}$ \\
& $\mathrm{bb}=200 \mathrm{ft}$, height $=5 \mathrm{ft}$ \\
& $\mathrm{bb}=400 \mathrm{ft}$, height $=5 \mathrm{ft}$ \\
& Meteorological Systems \\
& $\mathrm{bb}=50 \mathrm{ft}$, height $=5 \mathrm{ft}$ \\
& $\mathrm{bb}=100 \mathrm{ft}$, height $=5 \mathrm{ft}$ \\
\hline
\end{tabular}

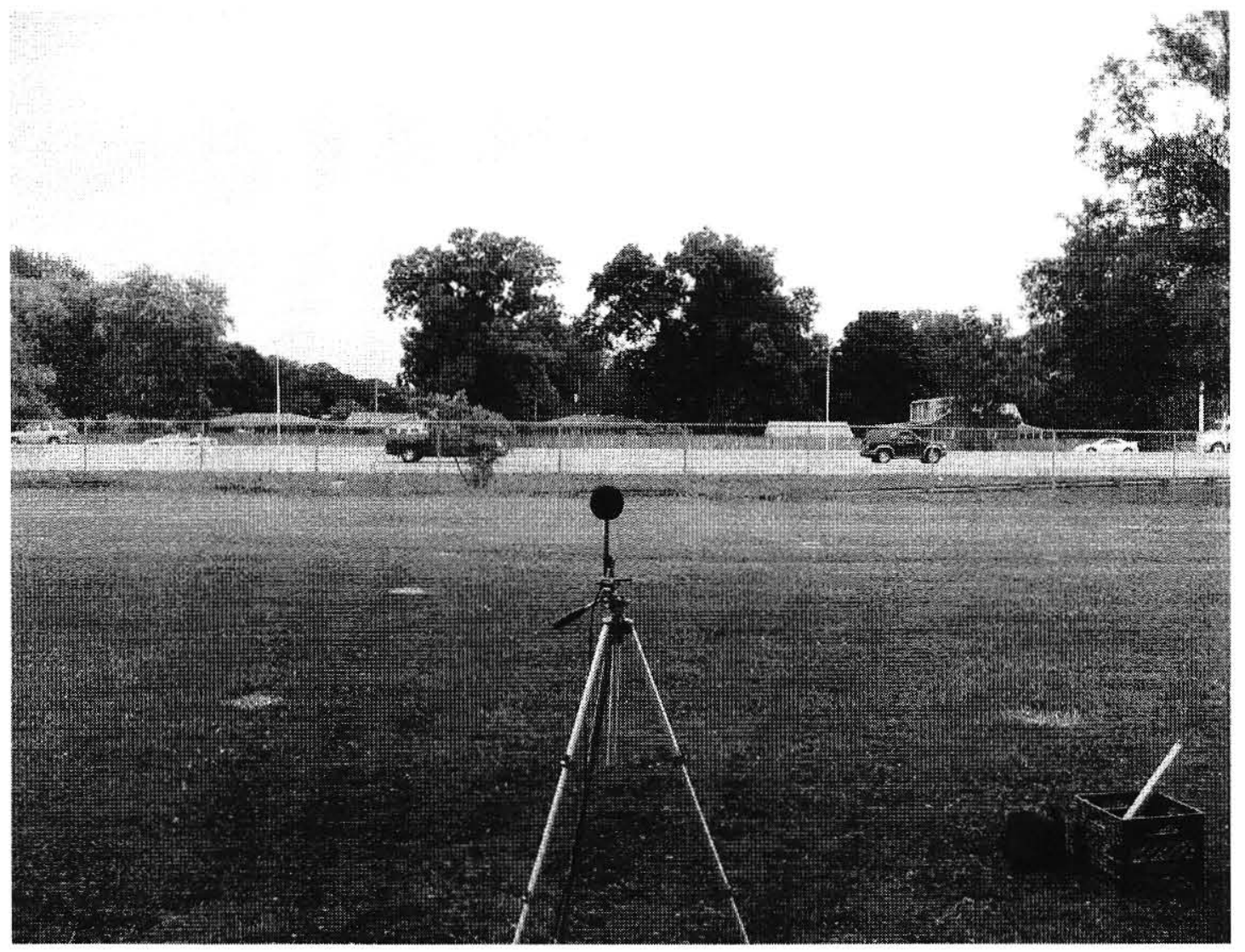

Figure 8-5. Site 03KY: Description and Photograph 


\begin{tabular}{|l|l|}
\hline Site ID & 04IN \\
\hline Location & Roadway 111, IN \\
\hline Site Type & Open, flat space \\
\hline Ground Type & Lawn \\
\hline Roadway & 2 lanes, shoulders \\
\hline Instrumentation & Microphones \\
Positions & $\mathrm{bb}=50 \mathrm{ft}$, height $=5 \mathrm{ft}$ \\
& $\mathrm{bb}=100 \mathrm{ft}$, height $=5 \mathrm{ft}$ \\
& $\mathrm{bb}=200 \mathrm{ft}$, height $=5 \mathrm{ft}$ \\
& Meteorological Systems \\
& $\mathrm{bb}=50 \mathrm{ft}$, height $=5 \mathrm{ft}$ \\
& $\mathrm{bb}=100 \mathrm{ft}$, height $=5 \mathrm{ft}$ \\
\hline
\end{tabular}

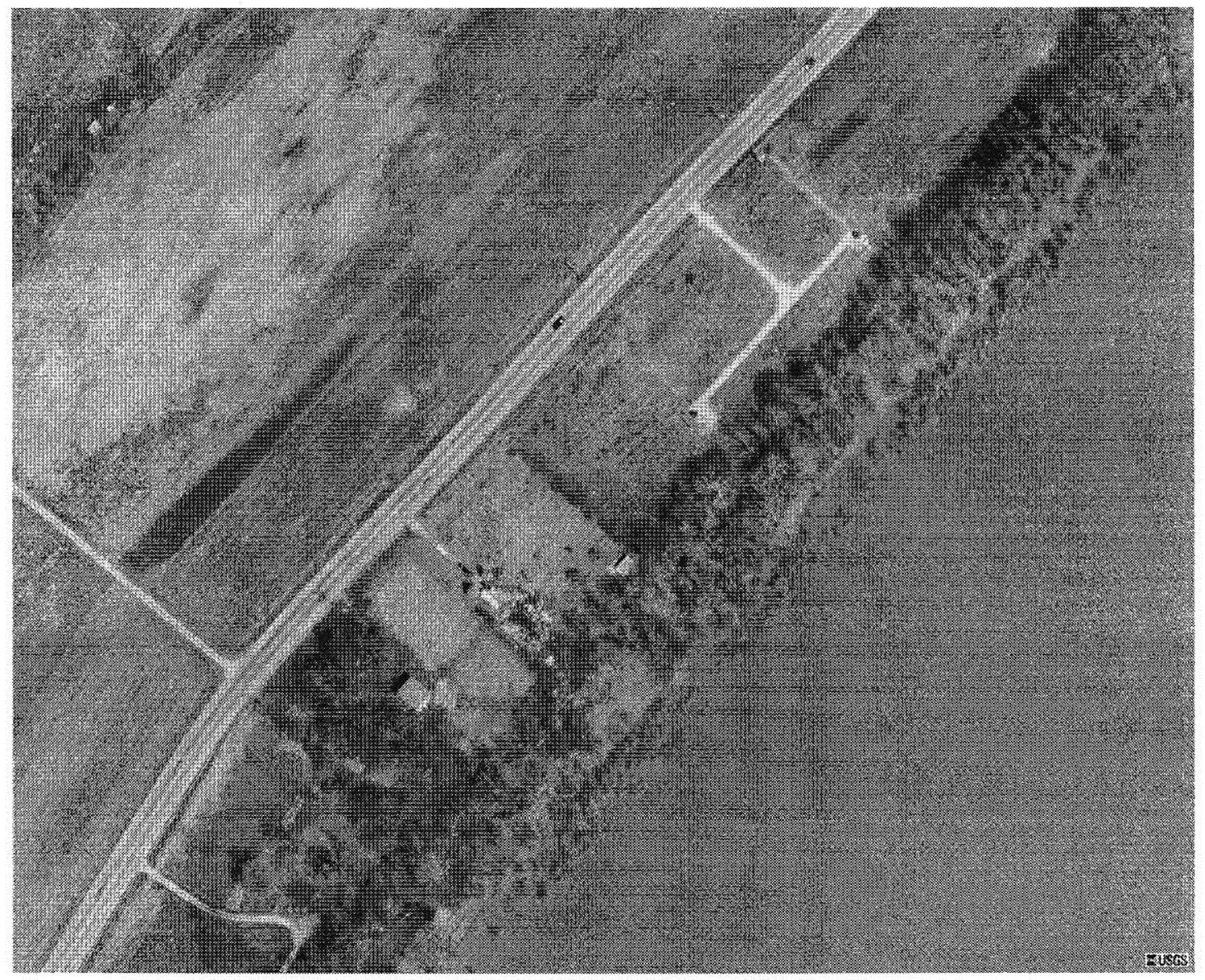

Figure 8-6. Site 04IN: Description and Photograph 


\begin{tabular}{|l|l|}
\hline Site ID & 04IN-TNM model (TNM Version 2.5) \\
\hline Default Ground Type & Lawn \\
\hline Pavement Type & Average \\
\hline TNM objects & roadways, receivers \\
\end{tabular}

Plan view

Skew view

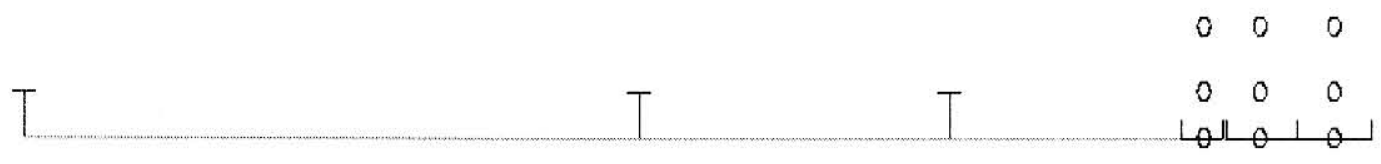

Figure 8-7. Site 04IN: TNM 2.5 Model Description, TNM Plan, Skew View 


\begin{tabular}{|c|c|}
\hline Site ID & $04 \mathrm{CT}$ \\
\hline Location & $\begin{array}{l}\text { East Hartford, CT;Rte 84; Northbound side; North of exit 58; } \\
\text { farthest North lawn of Woodcliff Estates }\end{array}$ \\
\hline Site Type & Barrier (17.3 ft wood), flat \\
\hline Ground Type & Lawn and some pavement, mixed acoustically soft and hard \\
\hline Roadway & 12 lanes, DGAC, shoulders, pavement median \\
\hline $\begin{array}{l}\text { Instrumentation } \\
\text { Positions }\end{array}$ & $\begin{array}{l}\text { Microphones } \\
\mathrm{D}=52.5 \mathrm{ft} \text { (offset from mic line, no barrier), } \\
\text { Height }=5 \mathrm{ft} \\
\mathrm{bb}=56 \mathrm{ft} \text {, height }=5 \text { and } 15 \mathrm{ft} \\
\mathrm{bb}=125 \mathrm{ft} \text {, height }=5 \text { and } 15 \mathrm{ft} \\
\mathrm{bb}=200 \mathrm{ft} \text {, height }=5 \text { and } 15 \mathrm{ft} \\
\text { Meteorological Systems } \\
\mathrm{bb}=75 \mathrm{ft} \text {, height }=5 \text { and } 15 \mathrm{ft} \\
\mathrm{bb}=175 \mathrm{ft} \text {, height }=5 \text { and } 15 \mathrm{ft}\end{array}$ \\
\hline
\end{tabular}

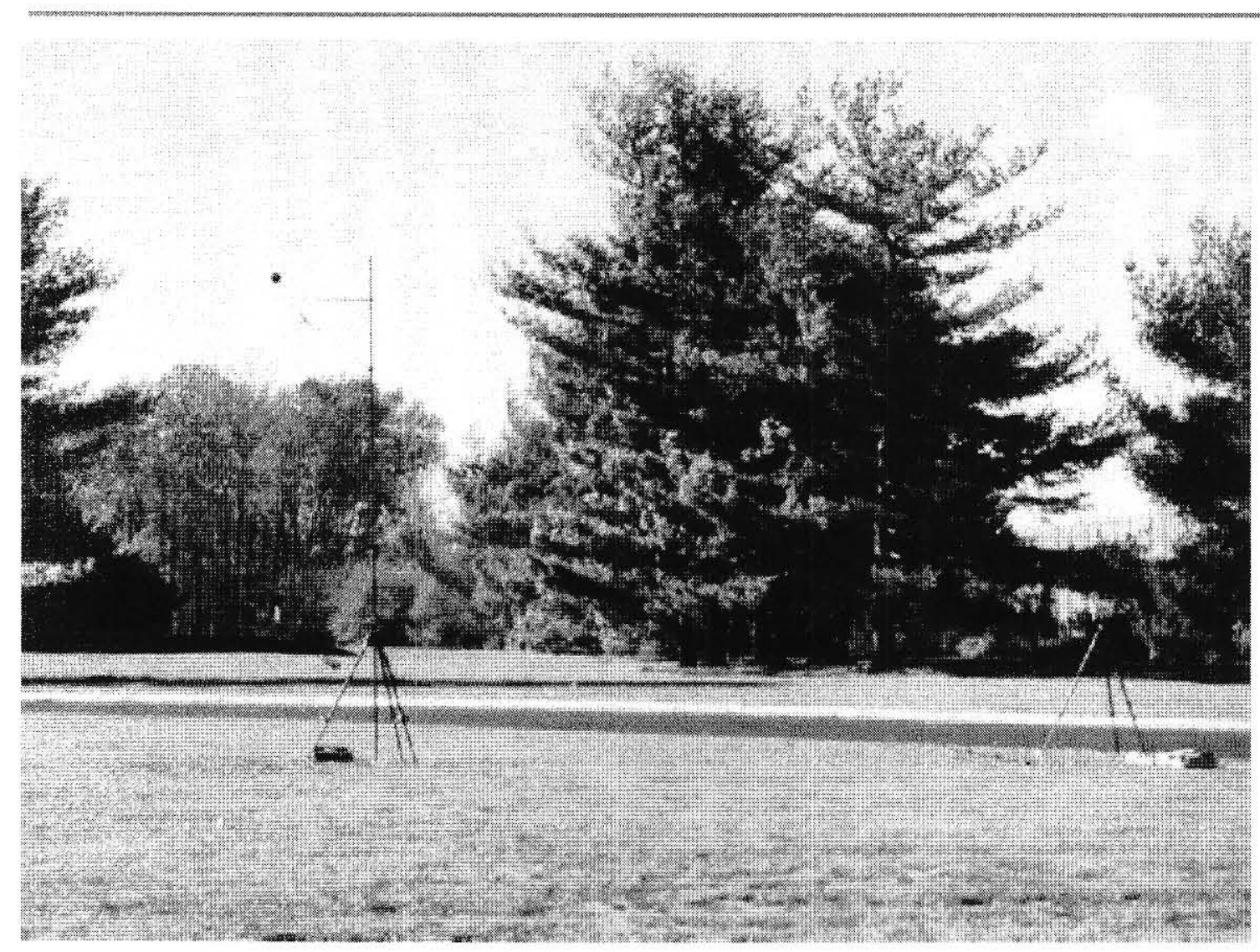

Figure 8-8. Site 04CT: Description and Photograph

Source: Validation of FHWA's Traffic Noise Model (TNM) (Rochat, 2002) 


\begin{tabular}{|l|l|}
\hline Site ID & 04CT-TNM model (TNM Version 2.5) \\
\hline Default Ground Type & Lawn \\
\hline Pavement Type & Average \\
\hline TNM objects & $\begin{array}{l}\text { Barrier, roadways, receivers, terrain line (change in } \\
\text { elevation of }-5 \mathrm{ft} \text { from barrier base }), \\
\text { Ground zone (pavement parking lot: largest width }=55 \mathrm{ft}, \\
\text { largest length } \sim 530 \mathrm{ft})\end{array}$ \\
\hline
\end{tabular}

Plan view

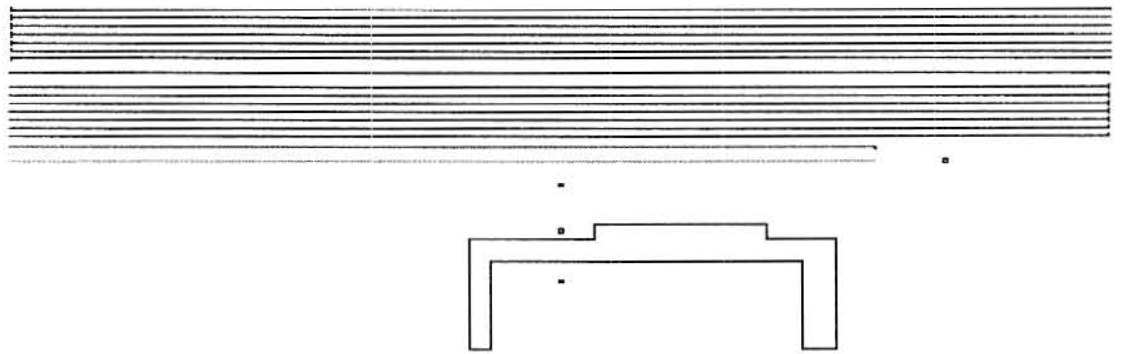

Perspective view

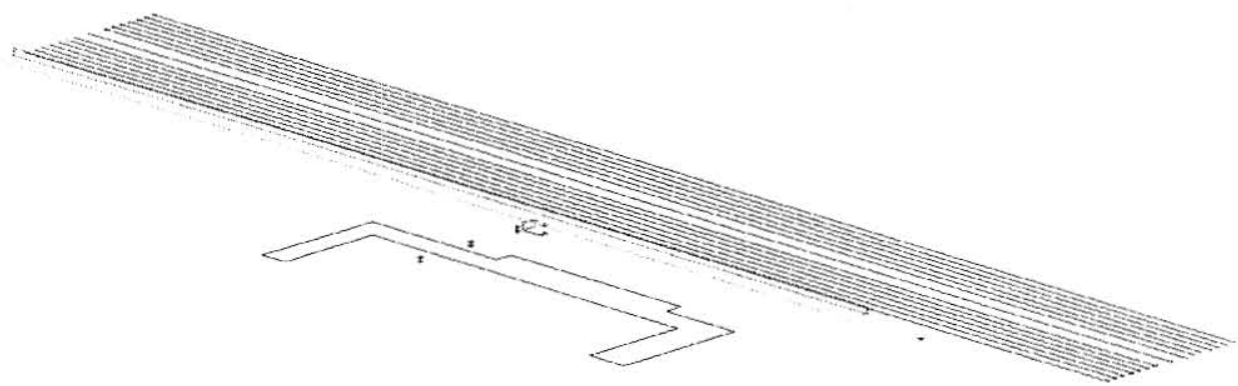

Skew view

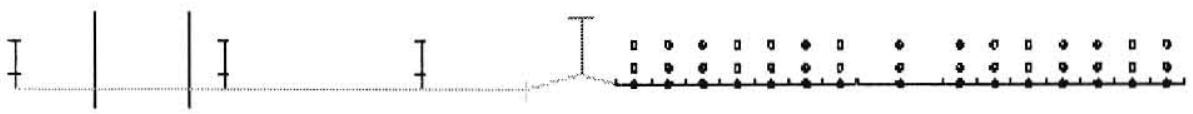

Figure 8-9. Site 04CT: TNM 2.5 Model Description, TNM Plan, Skew View and perspective view 


\begin{tabular}{|c|c|}
\hline Site ID & $05 \mathrm{CA}$ \\
\hline Location & $\begin{array}{l}\text { Chino Hills, CA; Rte 71; Southbound side; just North of } \\
\text { Central Ave/Soquel Cyn Pkwy Exit; near intersection of Los } \\
\text { Serranos and Pomona Ricon }\end{array}$ \\
\hline Site Type & Barrier ( $15 \mathrm{ft}$ concrete block), flat \\
\hline Ground Type & Field grass, acoustically soft \\
\hline Roadway & 8 lanes, PCC, shoulders, pavement median \\
\hline $\begin{array}{l}\text { Instrumentation } \\
\text { Positions }\end{array}$ & $\begin{array}{l}\text { Microphones } \\
\mathrm{bb}=0 \mathrm{ft}, \text { height }=3.5 \mathrm{ft} \text { above barrier } \\
\mathrm{bb}=50 \mathrm{ft} \text {, height }-5 \text { and } 15 \mathrm{ft} \\
\mathrm{bb}=100 \mathrm{ft} \text {, height }=5 \text { and } 15 \mathrm{ft} \\
\mathrm{bb}=150 \mathrm{ft} \text {, height }=5 \text { and } 15 \mathrm{ft} \\
\text { Meteorological Systems } \\
\mathrm{bb}=75 \mathrm{ft} \text {, height }=5 \text { and } 15 \mathrm{ft} \\
\mathrm{bb}=175 \mathrm{ft} \text {, height }=5 \text { and } 15 \mathrm{ft}\end{array}$ \\
\hline
\end{tabular}

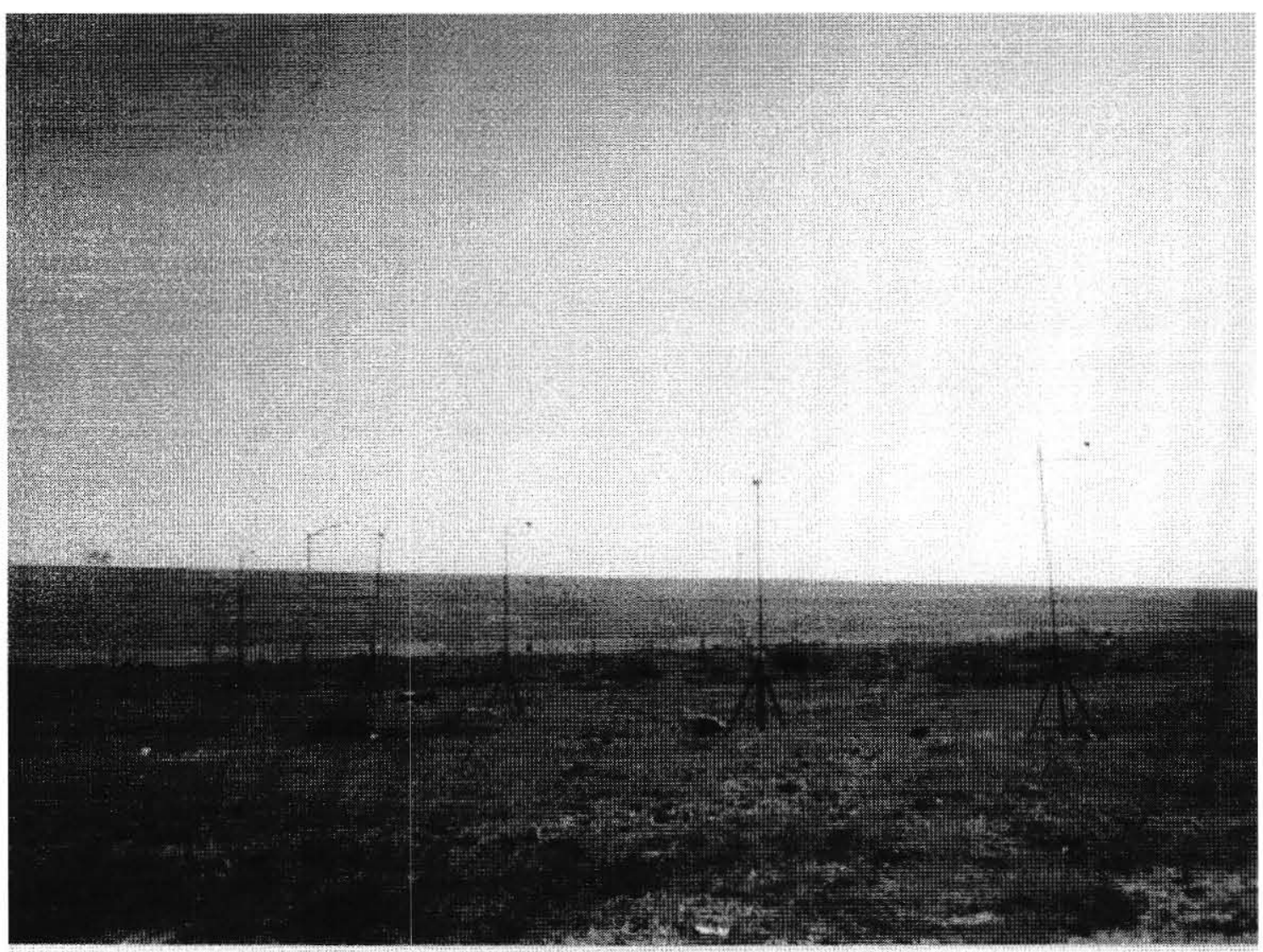

Figure 8-10. Site 05CA: Description and Photograph

Source: Validation of FHWA's Traffic Noise Model (TNM) (Rochat, 2002) 


\begin{tabular}{|l|l|}
\hline Site ID & 05CA-TNM model (TNM Version 2.5) \\
\hline Default Ground Type & Field grass \\
\hline Pavement Type & Average \\
\hline TNM objects & Barrier, roadways, receiver \\
\hline
\end{tabular}

Plan view

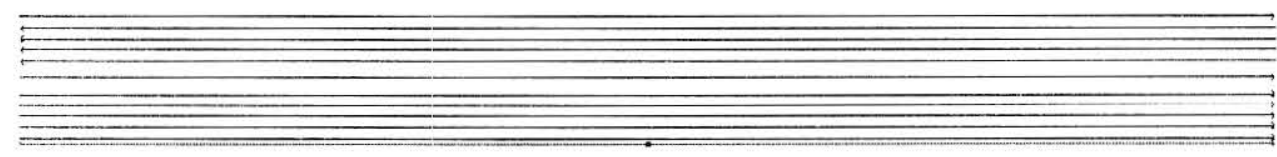

Perspective view

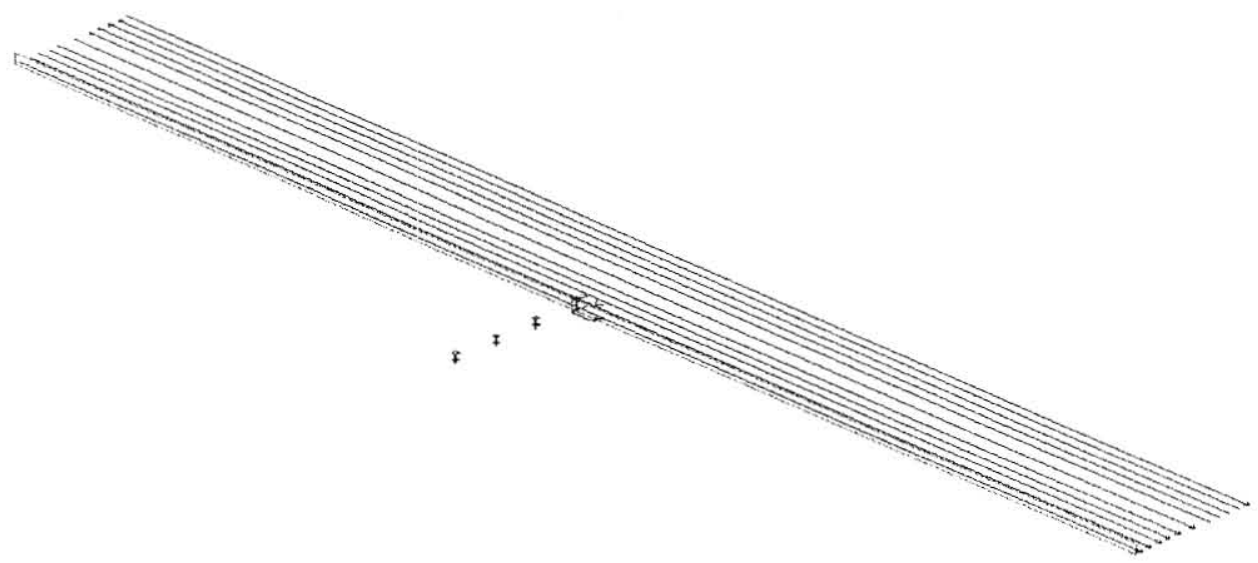

Skew view

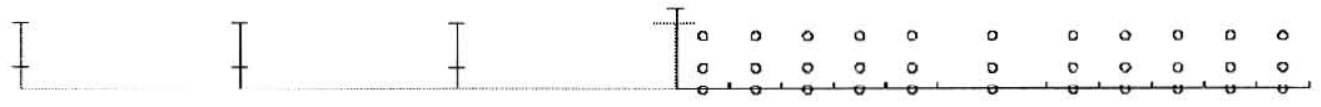

Figure 8-11. Site 05CA: TNM 2.5 Model Description, TNM Plan, skew view, and perspective view 


\begin{tabular}{|l|l|}
\hline Site ID & 08CA \\
\hline Location & $\begin{array}{l}\text { Anaheim, CA; Rte 91; Eastbound side; East of Lakeview Exit; } \\
\text { playing fields of Peralta Canyon Park }\end{array}$ \\
\hline Site Type & Barrier (14.5 ft concrete block), relatively flat \\
\hline Ground Type & Lawn, acoustically soft \\
\hline Roadway & $\begin{array}{l}\text { 14 lanes, PCC (HOV lanes DGAC), shoulders, pavement } \\
\text { median }\end{array}$ \\
\hline $\begin{array}{l}\text { Instrumentation } \\
\text { Positions }\end{array}$ & $\begin{array}{l}\text { Microphones } \\
\text { bb=0 ft, height }=5 \mathrm{ft} \text { above barrier } \\
\mathrm{bb}=50 \mathrm{ft} \text {, height }=5 \text { and } 15 \mathrm{ft} \\
\mathrm{bb}=200 \mathrm{ft} \text {, height }=5 \text { and } 15 \mathrm{ft} \\
\mathrm{bb}=30 \mathrm{ft} \text {, height }=5 \text { and } 15 \mathrm{ft}\end{array}$ \\
& $\begin{array}{l}\text { Meteorological Systems } \\
\mathrm{bb}=100 \mathrm{ft} \text {, height }=5 \text { and } 15 \mathrm{ft}\end{array}$ \\
\hline
\end{tabular}

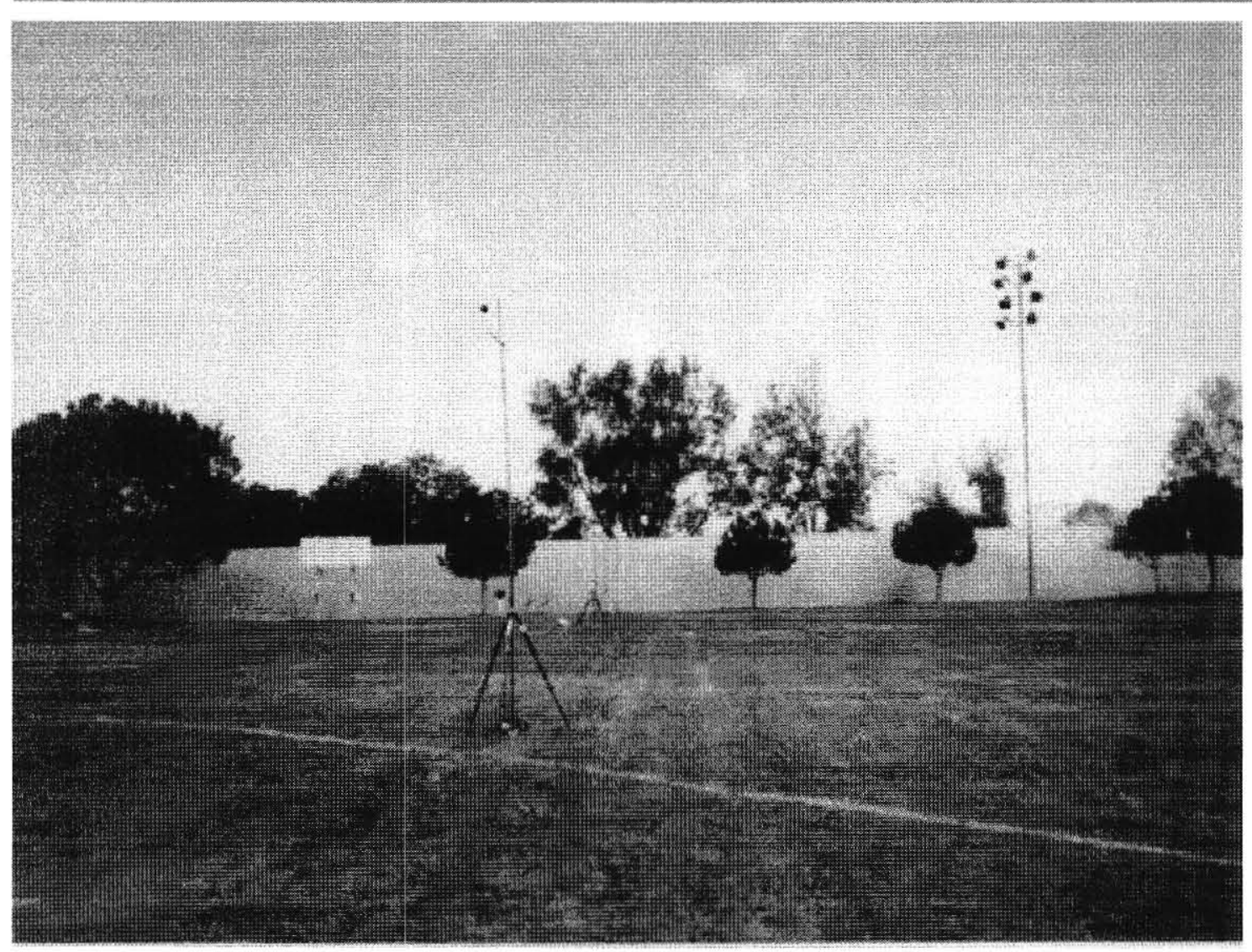

Figure 8-12. Site 08CA: Description and Photograph

Source: Validation of FHWA's Traffic Noise Model (TNM) (Rochat, 2002) 


\begin{tabular}{|l|l|}
\hline Site ID & 08CA-TNM model (TNM Version 2.5) \\
\hline Default Ground Type & Lawn \\
\hline Pavement Type & Average \\
\hline TNM objects & Barrier, roadways, receiver \\
\hline
\end{tabular}

Plan view

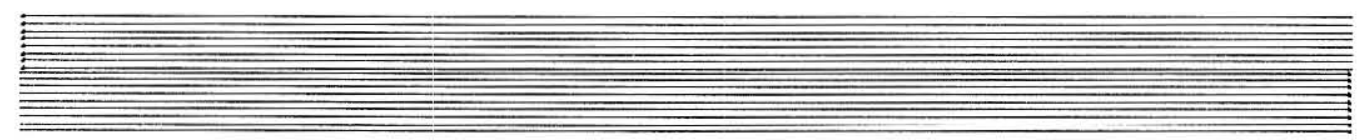

Perspective view

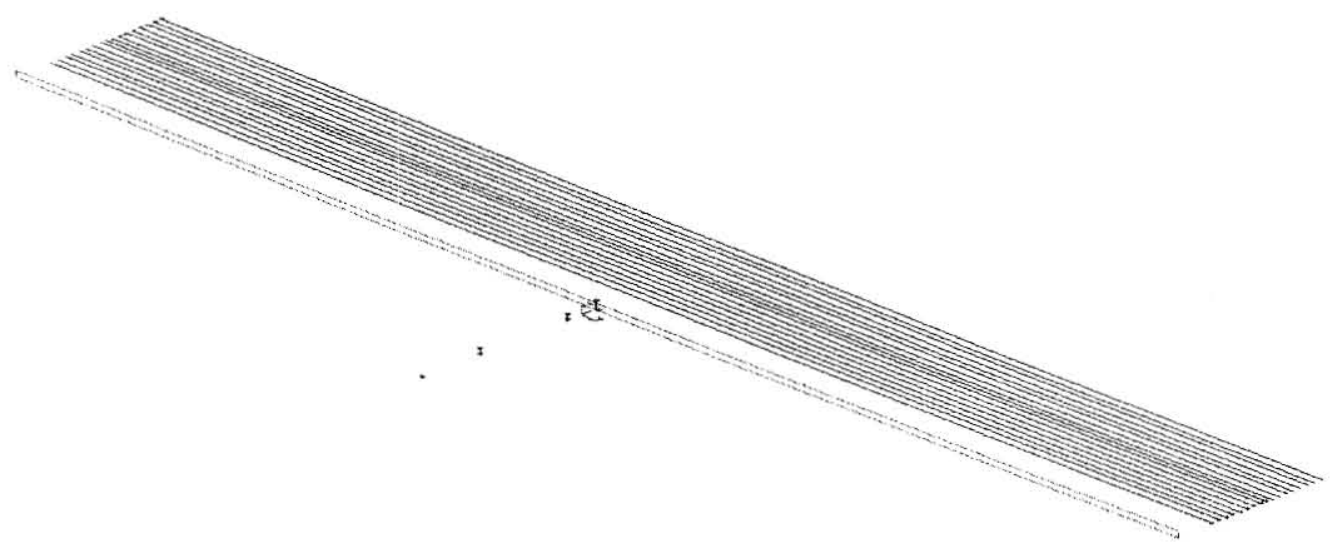

Skew view

T I 1 I

Figure 8-13. Site 08CA: TNM 2.5 Model Description, TNM Plan, skew view, and perspective view 


\begin{tabular}{|l|l|}
\hline Site ID & 09CA \\
\hline Location & $\begin{array}{l}\text { Chino, CA; Rte 71; Northbound side; North of Edison/Grand } \\
\text { Exit; field at end of Alicia St }\end{array}$ \\
\hline Site Type & $\begin{array}{l}\text { Barrier (15 ft concrete block), flat, with 16 ft drop-off from } \\
\text { barrier }\end{array}$ \\
\hline Ground Type & Field grass, acoustically soft \\
\hline Roadway & 10 lanes, PCC, shoulders, pavement median \\
\hline $\begin{array}{l}\text { Instrumentation } \\
\text { Positions }\end{array}$ & $\begin{array}{l}\text { Microphones } \\
\text { bb=0 ft, height }=5 \mathrm{ft} \text { above barrier } \\
\mathrm{bb}=55 \mathrm{ft} \text {, height }=5 \text { and } 15 \mathrm{ft} \\
\mathrm{bb}=100 \mathrm{ft} \text {, height }=5 \text { and } 15 \mathrm{ft} \\
\mathrm{bb}=200 \mathrm{ft} \text {, height }=5 \text { and } 15 \mathrm{ft}\end{array}$ \\
& $\begin{array}{l}\text { Meteorological Systems } \\
\mathrm{bb}=75 \mathrm{ft}, \text { height }=5 \text { and } 15 \mathrm{ft} \\
\mathrm{bb}=150 \mathrm{ft}, \text { height }=5 \text { and } 15 \mathrm{ft}\end{array}$ \\
\hline
\end{tabular}

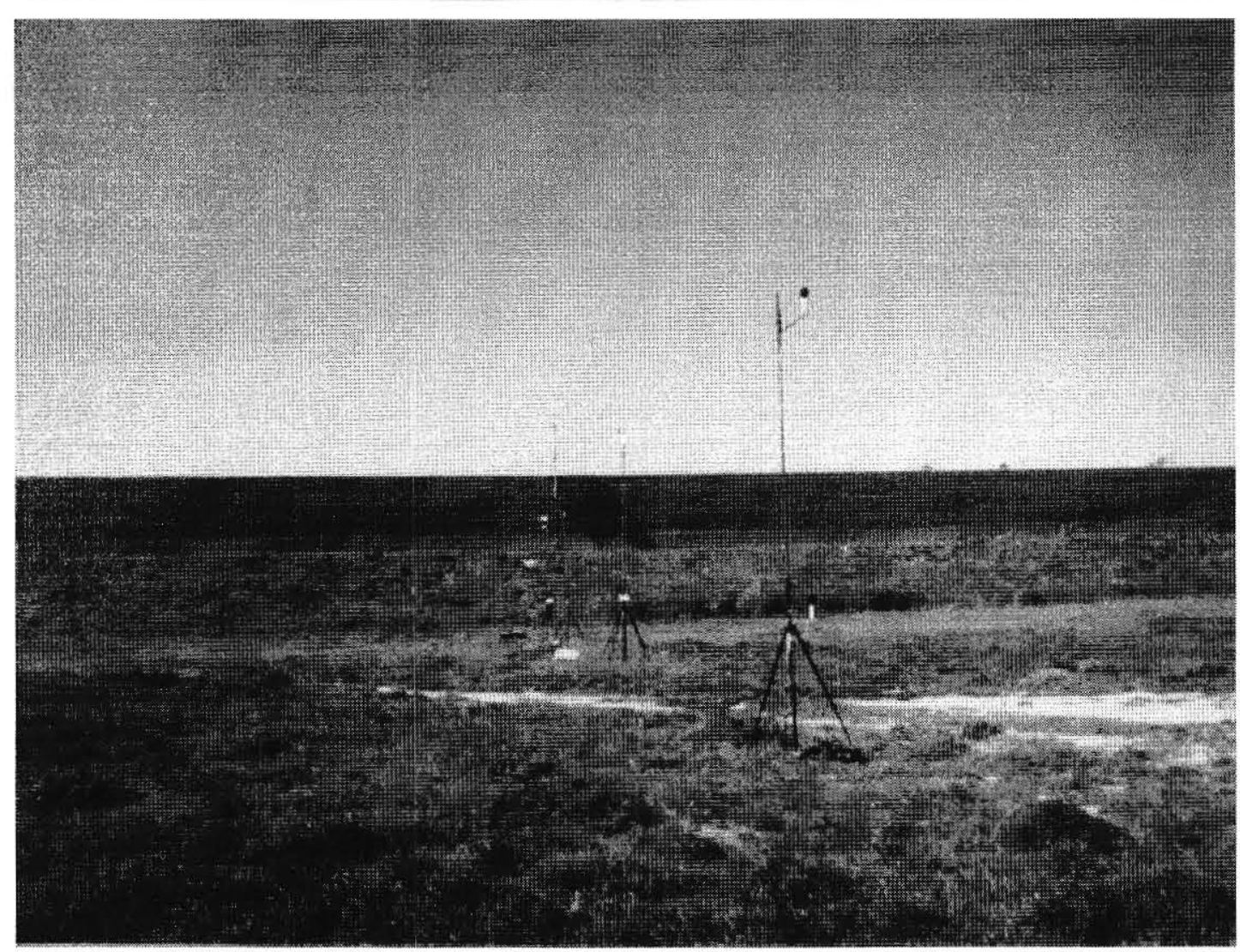

Figure 8-14. Site 09CA: Description and Photograph

Source: Validation of FHWA's Traffic Noise Model (TNM) (Rochat, 2002) 


\begin{tabular}{|l|l|}
\hline Site ID & 09CA-TNM model (TNM Version 2.5) \\
\hline Default Ground Type & Field grass \\
\hline Pavement Type & Average \\
\hline TNM objects & $\begin{array}{l}\text { Roadways, receivers, barrier, terrain line (change in } \\
\text { elevation of }-16 \mathrm{ft} \text { from barrier base to mic line) }\end{array}$ \\
\hline
\end{tabular}

Plan view

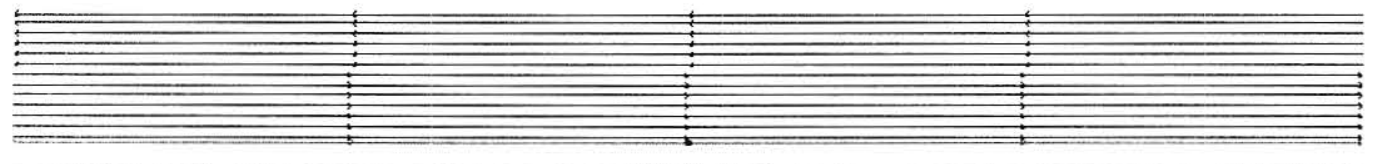

$\cdot$

Skew view

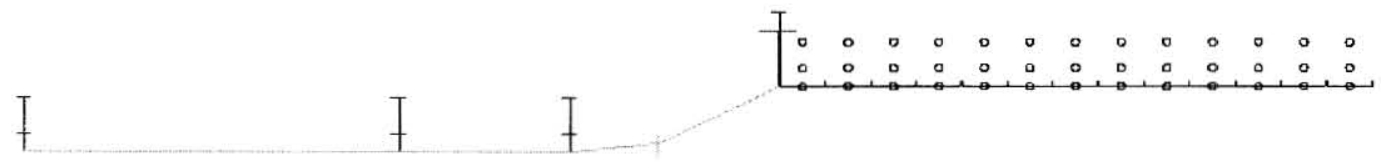

Perspective view

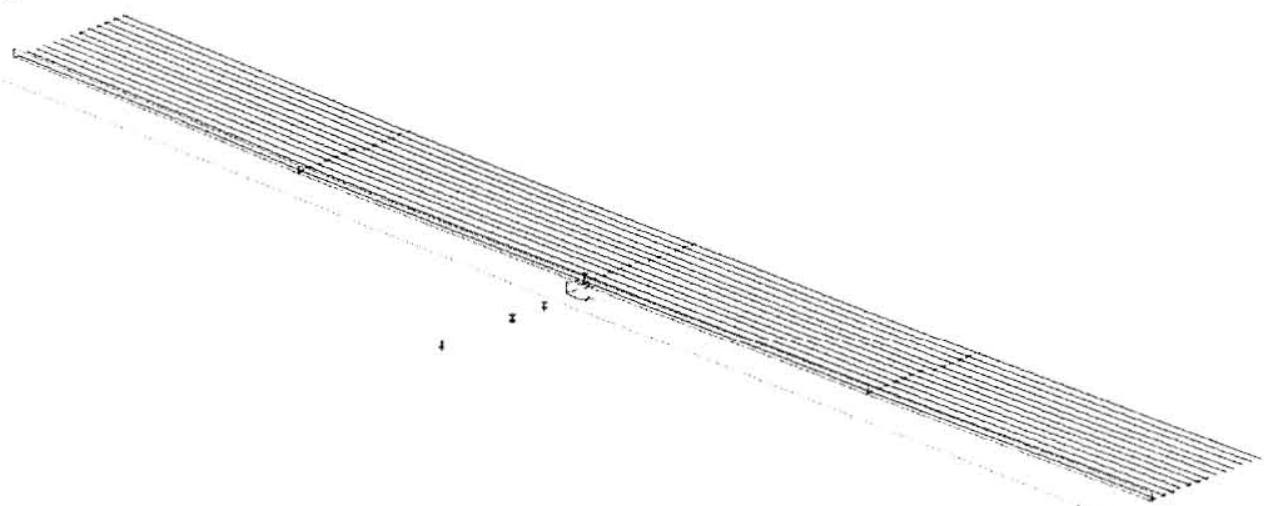

Figure 8-15. Site 09CA: TNM 2.5 Model Description, TNM Plan, Skew View and Perspective View 


\begin{tabular}{|l|l|}
\hline Site ID & 11CA \\
\hline Location & $\begin{array}{l}\text { Sunnyvale,CA; Rte 237; Westbound side; just East of E. } \\
\text { Carribean Drive Exit; Eastern end of Sunnyvale Baylands } \\
\text { County Park }\end{array}$ \\
\hline Site Type & Barrier (16 ft wood), relatively flat \\
\hline Ground Type & $\begin{array}{l}\text { Field grass and some pavement, mixed acoustically soft and } \\
\text { hard }\end{array}$ \\
\hline Roadway & $\begin{array}{l}\text { 3 lanes + 2 auxiliary lanes, DGAC, shoulders, buffer zones, } \\
\text { pavement median }\end{array}$ \\
\hline $\begin{array}{l}\text { Instrumentation } \\
\text { Positions }\end{array}$ & $\begin{array}{l}\text { Microphones } \\
\mathrm{bb}=0 \mathrm{ft} \text {, height }=5 \mathrm{ft} \text { above barrier } \\
\mathrm{bb}=50 \mathrm{ft} \text {, height }=5 \text { and } 15 \mathrm{ft} \\
\mathrm{bb}=100 \mathrm{ft}, \text { height }=5 \text { and } 15 \mathrm{ft} \\
\mathrm{bb}=300 \mathrm{ft} \text {, height }=5 \text { and } 15 \mathrm{ft}\end{array}$ \\
$\begin{array}{l}\text { Meteorological Systems } \\
\mathrm{bb}=75 \mathrm{ft}, \text { height }=5 \text { and } 15 \mathrm{ft} \\
\mathrm{bb}=200 \mathrm{ft}, \text { height }=5 \text { and } 15 \mathrm{ft}\end{array}$ \\
\hline
\end{tabular}

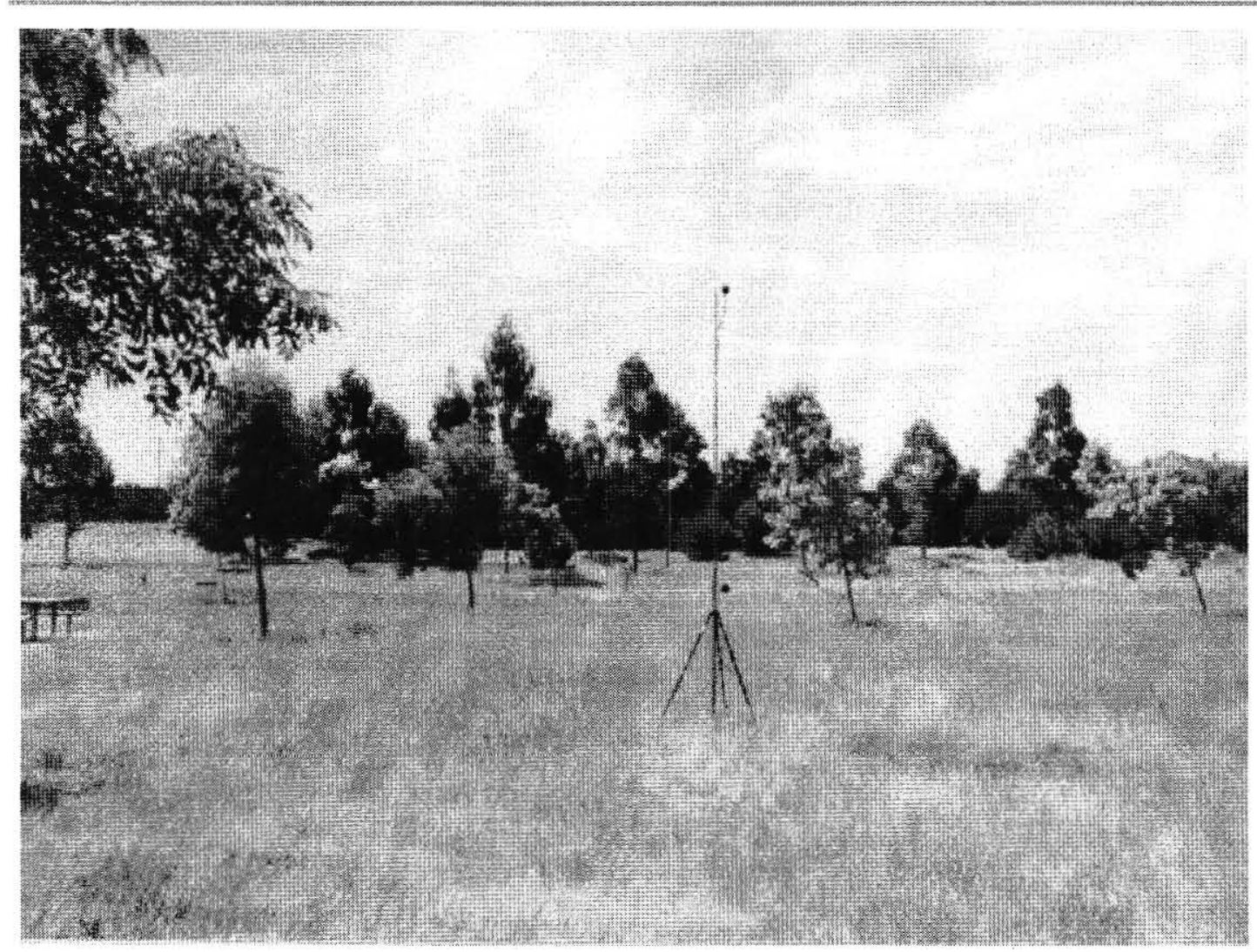

Figure 8-16. Site 11CA: Description and Photograph

Source: Validation of FHWA's Traffic Noise Model (TNM) (Rochat, 2002) 


\begin{tabular}{|l|l|}
\hline Site ID & 11CA-TNM model (TNM Version 2.5) \\
\hline Default Ground Type & Field grass \\
\hline Pavement Type & Average \\
\hline TNM objects & $\begin{array}{l}\text { Roadways, receivers, barrier, terrain lines (change in } \\
\text { elevation of }-4 \mathrm{ft} \text { from barrier base/roadway level to mic } \\
\text { line), ground zone (pavement drive and parking area: largest } \\
\text { width =52 ft) }\end{array}$ \\
\hline
\end{tabular}

Plan view

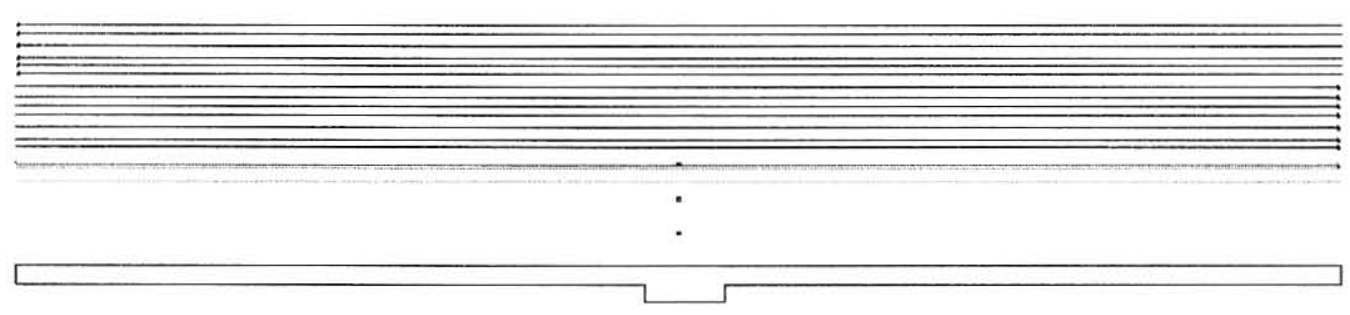

Perspective view

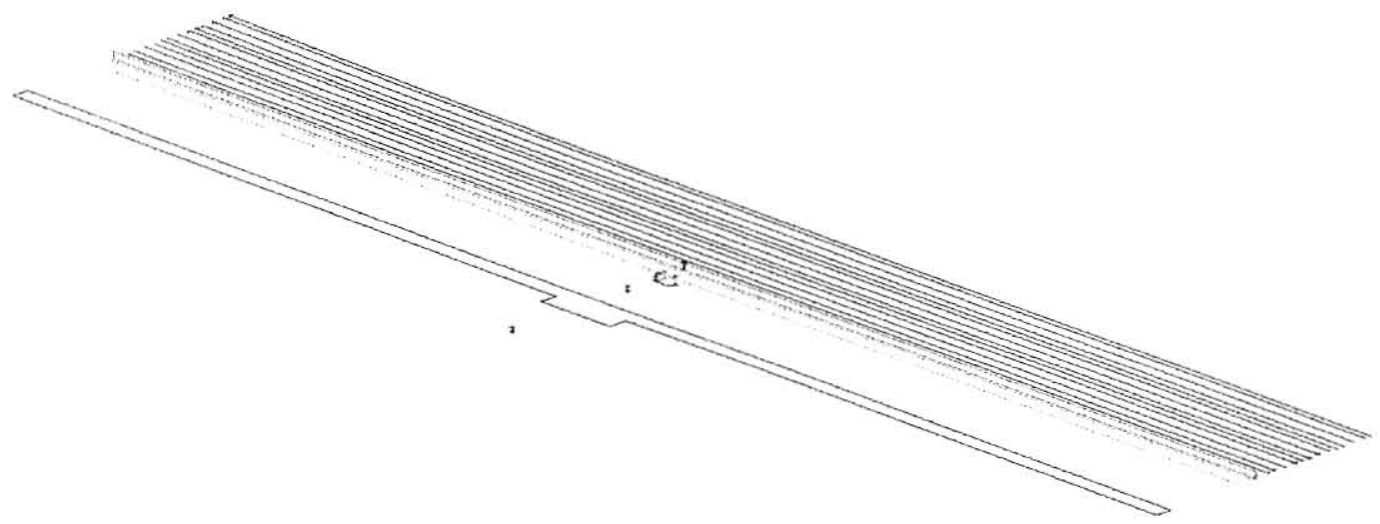

Skew view

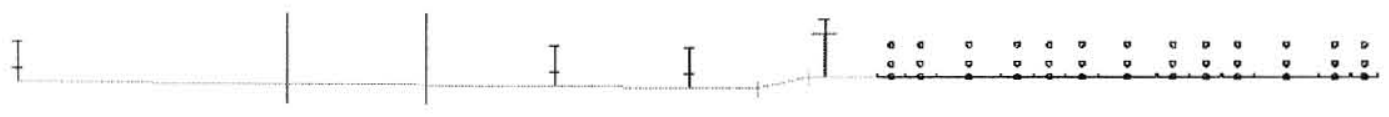

Figure 8-17. Site 11CA: TNM 2.5 Model Description, TNM Plan, Skew View and Perspective View 


\begin{tabular}{|l|l|}
\hline Site ID & 01MA \\
\hline Location & $\begin{array}{l}\text { Taunton, MA;Rte 24; Southbound side; just North of Exit 12, } \\
\text { near overpass }\end{array}$ \\
\hline Site Type & Open area, flat \\
\hline Ground Type & Field grass, acoustically soft \\
\hline Roadway & 4 lanes, DGAC, shoulders, field grass median \\
\hline Instrumentation & Microphones \\
Positions & $\mathrm{d}=50 \mathrm{ft}$, height $=5$ and $15 \mathrm{ft}$ \\
& $\mathrm{d}=100 \mathrm{ft}$, height $=5$ and $15 \mathrm{ft}$ \\
& $\mathrm{d}=200 \mathrm{ft}$, height $=5$ and $15 \mathrm{ft}$ \\
& \\
& Meteorological Systems \\
& $\mathrm{D}=75 \mathrm{ft}$, height $=5$ and $15 \mathrm{ft}$ \\
& $\mathrm{D}=150 \mathrm{ft}$, height $=5$ and $15 \mathrm{ft}$ \\
\hline
\end{tabular}

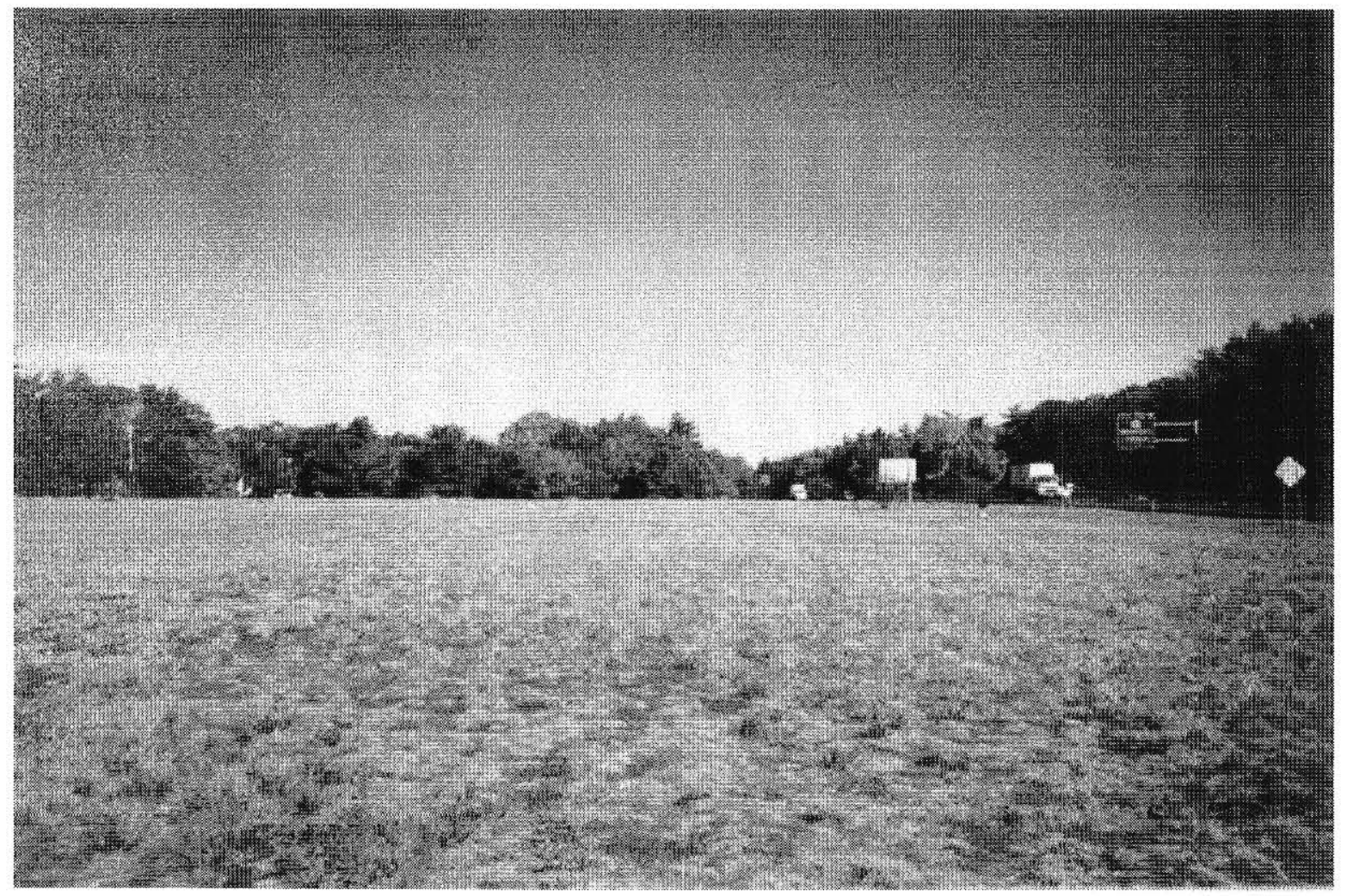

Figure 8-18. Site 01MA: Description and Photograph

Source: Validation of FHWA's Traffic Noise Model (TNM) (Rochat, 2002) 


\begin{tabular}{|l|l|}
\hline Site ID & 01MA-TNM model (TNM Version 2.5) \\
\hline Default Ground Type & Field grass \\
\hline Pavement Type & Average \\
\hline TNM objects & $\begin{array}{l}\text { Roadways, receivers, terrain lines (defining trench: depth =4 } \\
\text { ft) }\end{array}$ \\
\hline
\end{tabular}

Plan view

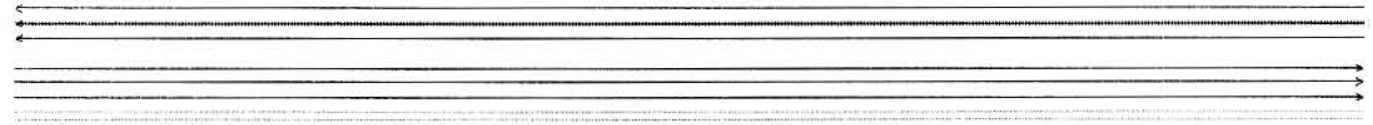

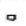

o

Perspective view

$+\sum_{+}^{*}$

F

Skew view

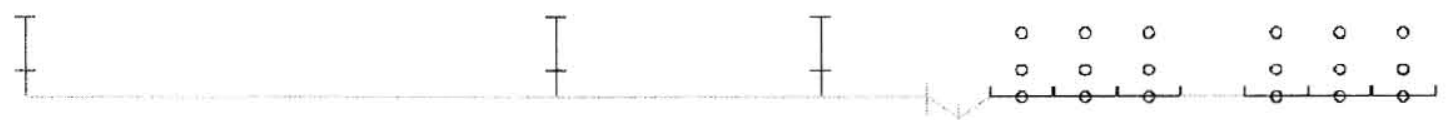

Figure 8-19. Site 01MA: TNM 2.5 Model Description, TNM Plan, Skew View and Perspective View 


\section{CURRICULUM VITAE}

NAME:

PERMANENT ADDRESS:

CURRENT ADDRESS:

DOB:

EDUCATION \& TRAINING:
Ning Shu

16-8A COTE D'AZUR

Shenzhen, GuangDong, 518042, P.R.China

777 Theodore Burnett Ct. \#2

Louisville, KY, 40217

July 7, 1975, Guangzhou, GruangDong, P.R.China

B.S., Transportation Engineering

South China University of Technology

Guangzhou, P.R.China

September, 1994 -- July, 1998

M.S., Control Theory and Control Engineering

South China University of Technology

Guangzhou, P.R.China

September, 1998 - March, 2001

Ph.D., Civil \& Environmental Engineering

University of Louisville, Kentucky

September, 2002 - May, 2005

PROFESSIONAL SOCIETIES: Teaching and Research Assistance

College of Traffic \& Communications

South China University of Technology, Guangzhou

September, 1999 - March, 2001

University of Louisville Fellowship

University of Louisville, Kentucky

September, 2002 - August, 2004

Research Assistance

Dept. of Civil \& Environmental Engineering

University of Louisville, Kentucky

September, 2004 - May, 2005 